FERNANDA BALEN

\title{
EM BUSCA DE NOVOS MÉTODOS DE TRATAMENTO \\ PARA A RETINOSE PIGMENTAR CAUSADA POR \\ MUTAÇÕES NA RODOPSINA
}

Tese apresentada ao Programa de PósGraduacão em Biologia Celular e Tecidual do Instituto de Ciências Biomédicas da Universidade de São Paulo, para obtenção do Título de Doutor em Ciências. 


\section{EM BUSCA DE NOVOS MÉTODOS DE TRATAMENTO \\ PARA A RETINOSE PIGMENTAR CAUSADA POR \\ MUTAÇÕES NA RODOPSINA}

Tese apresentada ao Programa de PósGraduacão em Biologia Celular e Tecidual do Instituto de Ciências Biomédicas da Universidade de São Paulo, para obtenção do Título de Doutor em Ciências.

Área de concentração: Biologia Celular e Tecidual

Orientadora: Prof ${ }^{\mathrm{a}}$. Dr ${ }^{\mathrm{a}}$. Dânia Hamassaki

Co-orientadora: Prof ${ }^{\mathrm{a}}$. Dr ${ }^{\mathrm{a}}$. Judith KleinSeetharaman

Versão corrigida. Versão original eletrônica encontra-se disponível tanto na biblioteca do ICB quanto na biblioteca digital de teses e dissertações da USP (bdtd). 


\section{DADOS DE CATALOGAÇÃO NA PUBLICAÇÄO (CIP) \\ Serviço de Biblioteca e Informação Biomédica do Instituto de Ciências Biomédicas da Universidade de Säo Paulo \\ reproduçäo näo autorizada pelo autor}

Balen, Fernanda.

Em busca de novos métodos de tratamento para a retinose pigmentar causada por mutações na rodopsina / Fernanda Balen. São Paulo, 2012.

Orientador: Profa. Dra. Dânia Emi Hamassaki.

Tese (Doutorado) - Universidade de São Paulo. Instituto de Ciências Biomédicas. Departamento de Biologia Celular e do Desenvolvimento. Área de concentração: Biologia Celular e Tecidual. Linha de pesquisa: Degenaração e regeneração de retina.

Versão do título para $\circ$ inglês: Finding new approaches to treat retinitis pigmentosa caused by mutations in the photoreceptor rhodopsin.

1. Retina 2. Fotorreceptores 3. Degeneração retiniana I. Hamassaki, Profa. Dra. Dânia Emi II. Universidade de São Paulo. Instituto de Ciências Biomédicas. Programa de Pós-Graduação em Biologia Celular e Tecidual III. Título. 


\begin{abstract}
Candidato(a):
Fernanda Balen.

Título da Tese:

Em busca de novos métodos de tratamento para a retinose pigmentar causada por mutações na rodopsina.
\end{abstract}

Orientador(a):

Profa. Dra. Dânia Emi Hamassaki.

A Comissão Julgadora dos trabalhos de Defesa da Tese de Doutorado, em sessão pública realizada a considerou
( ) Aprovado(a)
( ) Reprovado(a)

\begin{tabular}{|c|c|}
\hline Examinador(a): & $\begin{array}{l}\text { Assinatura: } \\
\text { Nome: ......... } \\
\text { Instituição: . }\end{array}$ \\
\hline Examinador(a): & $\begin{array}{l}\text { Assinatura: } \\
\text { Nome: ........ } \\
\text { Instituição: . }\end{array}$ \\
\hline Examinador(a): & $\begin{array}{l}\text { Assinatura: } \\
\text { Nome: ......... } \\
\text { Instituição: . }\end{array}$ \\
\hline Examinador(a): & $\begin{array}{l}\text { Assinatura: } \\
\text { Nome: ........ } \\
\text { Instituição: . }\end{array}$ \\
\hline Presidente: & $\begin{array}{l}\text { Assinatura: } \\
\text { Nome: ........ } \\
\text { Instituição: . }\end{array}$ \\
\hline
\end{tabular}




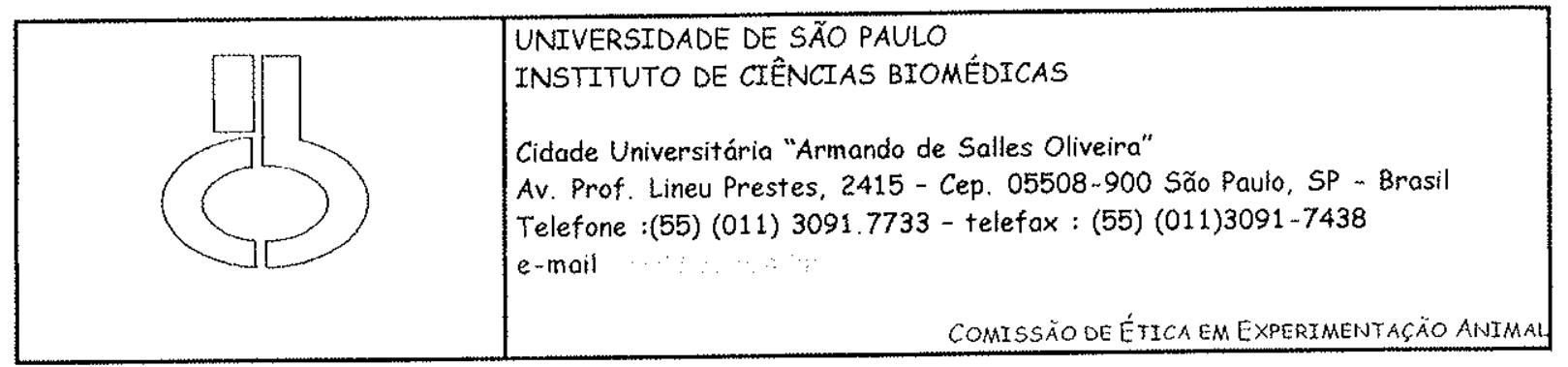

Decl. CEEA.054.08

\section{DECLARAÇ̃̃O}

Em adendo ao Certificado 52/05/CEEA, datado de 02.06 .05 e por solicitação da Profa. Dra. DANIA EMI HAMASSAKI, responsável pela linha de Pesquisa, autorizo a inclusão dos alunos Cinthia Takeyama, Felipe Monteleone Vieceli, Fernanda Balem, Loreni Cristine da Silva, Ricardo Moraes Borges a linha de pesquisa "Desenvolvimento, degeneração da retina de vertebrados: aspectos celulares e moleculares", uma vez que se trata de utilização da mesma espécie animal e de métodos experimentais similares ao referido certificado.

São Paulo, 23 de outubro de 2008.

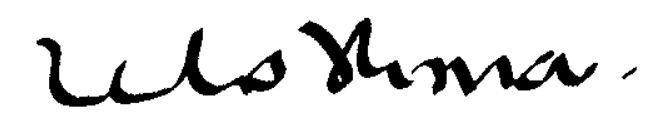

Prof. Dr. WOTHAN TAVARES DE LIMA Coordenador da CEEA- ICB/USP 
Dedicated

To My Family,

In Special To My Father

Celio 


\section{ACKNOWLEDGMENTS}

I express my deepest gratitude to my advisors Profs. Judith Klein-Seetharaman and Dânia Emi Hamassaki. Prof. Judith for granting me a unique opportunity in her lab as a pre-doctor fellow and as a $\mathrm{PhD}$ student, but also for her support, trust and unconditional help at all times. Prof. Dânia for giving me the chance to do my $\mathrm{PhD}$ with her, and for encouraging me every time I needed to do something that seemed to be almost impossible. Thank you so much you two for all advice related to my thesis or personal. Thanks a lot for all social celebrations we had together during Christmas, Halloween, B-days, as well day-to-day. I will always remember those good times.

I would like to thank all my "American” colleagues David Man, Hussein Baradia, Madhavi Ganapathiraju, Yanjun Qi, Neelima Mulpuri, Eric Gardner, Jenny Wu, Sylvia Schleker, Nicole Müller, Raghavan Balachandran, Arpana Dutta, Leelavathi Murthy, Subhodeep Moitra, Ivan Budyak, Meghana Kshirsagar, Kalyan Tirupula and in special to Harpreet Dhiman, Oznur Tastan, and Naveena Yanamala. Harpreet for all technical teaching and friendship, lunch time was not the same without you in the Lab. Oznur for being the best company during coffee-breaks, parties and excellent sport-mate. Naveena for all Lab aid, but most importantly, the time we spent both in lunch and coffee-breaks trying to solve all our $\mathrm{PhD}$ problems.

To all my "Brazilian” colleagues Sayami Akamine, Kelly Saito, Cristiano Pessoa, Loreni Cristine da Silva, Marcio Roberto Octávio Goncalves, and Leandro Mantovani, muito obrigada! Life in São Paulo was so much better with all of you around. Special thanks to Leandro that helped so much and made the importation of "my rats" so smooth. Keke, thanks for being so kind, friendly, and an excellent taxi driver! And Sasazita, I am afraid I cannot fully express my gratitude to you, but I can truly tell that without you this thesis would be only half brigadinho por tudo!!!!!

Thanks to Prof. Matthew M. LaVail and Lab personnel for the donation and help with the importation process of the transgenic rats. Prof. Patricia Gamma, Claudio and Marley, for the help, care and allocation of an especial room to "my rats". Prof. Luiz R. G. de Britto and Adilson for the availability and technical help with the microscopes. To Prof. Dora Fix Ventura for opening the doors of her Lab and Gabriela Loureçon Ioshimoto and Balázs Nagy for the support with the ERG experiments. I also appreciate 
the help of all administrative personal from both University of São Paulo and University of Pittsburgh.

I would like to acknowledge the DAAD-Helmholtz together with the Research Center Juelich, Germany, in special to Prof. Georg Bueldt, for supporting my PhD studies with a Pre-doctoral Fellowship.

I am very fortunate to have so many friends during my stay in Pittsburgh and São Paulo, thank you all of you for the great time we spent together.

I finally would like to extent my gratitude to my family. Pai, mesmo não estando aqui, saibas que estas sempre no meu pensamento. Mãezinha, Débora, Marcus Vinícius e Matheus, amo vocês incondicionalmente! Also to my extended family Maria Luiza and Nelso. Obrigada a todos por estarem sempre presentes.

To my long-time boyfriend, fiancé and husband Rodrigo! Ro, thank you so much for being part of my life. This thesis is certainly here because of you! Te amo! 


\begin{abstract}
Balen F. Finding new approaches to treat retinitis pigmentosa caused by mutations in the photoreceptor rhodopsin [Ph.D. Thesis (Cell and Developmental Biology)]. São Paulo: Instituto de Ciências Biomédicas, Universidade de São Paulo; 2012.
\end{abstract}

Retinitis Pigmentosa (RP) is an inherited disease that progressively leads to blindness. To date, more than 150 mutations associated with RP are known in rhodopsin. In vitro studies have shown that most of these mutations cause misfolding of rhodopsin. It has been hypothesized that molecular instability of the rhodopsin structure is responsible for disease severity in patients, but there is still no effective therapy to treat RP. Administration of vitamin A or retinoid derivatives is being used to rescue correctly folded rhodopsin and to slow down the degeneration, however, this treatment alone cannot cure RP. The focus of this thesis was to test the hypothesis that molecules other than retinal can help to rescue folded rhodopsin and/or reduce photoreceptor cell death. For that, the binding of other ligands to rhodopsin, was investigated in vitro, by studying the effects of the ligands on WT rhodopsin as well engineered rhodopsin mutants, and in vivo by making use of different rat models of RP. The major findings of this thesis are: I) The RP mutations, Asn-15-Ser (N15S) and Pro-23-His $(\mathrm{P} 23 \mathrm{H})$ were studied and characterized in vitro. Expression of these mutants in the presence of 9-cis and 11-cis retinal reveals that they differ in characteristics and severity, despite their global classification into the same class. $\mathrm{N} 15 \mathrm{~S}$ is slightly defect in structure, stability and cellular localization. $\mathrm{P} 23 \mathrm{H}$, on the other hand, is severely impaired at the molecular and cellular levels. II) Binding of small molecules, namely metal ions $\left(\mathrm{Zn}^{2+}, \mathrm{Cu}^{2+}, \mathrm{Fe}^{2+}, \mathrm{Ni}^{2+}, \mathrm{Mg}^{2+}\right.$ and $\left.\mathrm{Mn}^{2+}\right)$, the anthocyanin cyanidin-3-glucoside (C3G) and the chlorophyll-derivative chlorin e6 (Ce6), was tested. It was shown that these ligands directly interact with rhodopsin in vitro. Biophysical evidence indicated differential effects of these ligands on rhodopsin function, structure and dynamics. Ce6 was shown to be the best candidate to confer stability to the rhodopsin protein in vitro. III) Assessment of the effects of $\mathrm{Ce} 6$ on the stability of rhodopsin was tested in vivo: (a) First, normal rats, Sprague Dawley (SD), were subjected to lightdamage. Treatment with Ce6 appears to have only a minor effect on prevention of retinal damage. (b) Secondly, the effects of Ce6 on RP progression in vivo were evaluated in the P23H and Ser334ter (S334ter) rat models. Histological and functional analysis indicated 
that Ce6 seems to exert a positive functional effect by slowing the rate of $\mathrm{P} 23 \mathrm{H}$ photoreceptor degeneration in vivo. In contrast, Ce6 increased the photoreceptor degeneration of the S334ter rat in vivo. Collectively, the studies presented in this thesis enhance the knowledge related to several RP mutations, namely P23H, N15S and S334ter, which were found to cause misfolding of the photoreceptor rhodopsin in vitro and/or mediate the RP disease in vivo. It also conveys a new possibility for treatment of the RP disease with the identification of molecules (retinals, divalent metal ions, porphyrins and anthocyanins) that could aid in the stability and folding and modulate photoreceptor structure and function, thus paving the way to selectively target this receptor and aid directly in vision-rescue strategies.

Key-words: Retinitis Pigmentosa. Rhodopsin. Photoreceptors. 


\section{RESUMO}

Balen F. Em busca de novos métodos de tratamento para a retinose pigmentar causada por mutações na rodopsina [Tese (Doutorado em Biologia Celular e Tecidual)]. São Paulo: Instituto de Ciências Biomédicas, Universidade de São Paulo; 2012.

Retinose Pigmentar (RP) é uma doença hereditária que progressivamente conduz à cegueira. Atualmente, são conhecidas mais de 150 mutações da rodopsina. Estudos in vitro mostraram que a maioria destas mutações altera a conformação da rodopsina sugerindo que a instabilidade molecular da estrutura da rodopsina é responsável pela gravidade da RP em pacientes. Todavia, uma terapia efetiva para tratar a RP ainda não foi estabelecida. Administração de vitamina A ou derivados de retinóides são usados como tratamento para mediar a correta formação da rodopsina ou reduzir a degeneração. Contudo, o uso exclusivo desse tratamento não é suficiente para curar a RP. O objetivo desta tese foi testar a hipótese de que outras moléculas, além dos retinóides, poderiam influenciar na conformação correta da rodopsina e/ou reduzir a morte dos fotorreceptores. Com este objetivo, foi investigada in vitro e in vivo a ligação de outros compostos moleculares à rodopsina. Os efeitos destes compostos foram estudados in vitro na rodopsina selvagem e mutantes, e in vivo, utilizando diferentes modelos de ratos. Os principais resultados desta tese são: I) As mutações da RP Asn-15-Ser (N15S) e Pro-23His $(\mathrm{P} 23 \mathrm{H})$, foram estudadas e caracterizadas in vitro. A expressão destas mutações na presença de retinóides 9-cis ou 11-cis revelou que ambas apresentam diferenças em características e severidade, apesar de serem globalmente classificados dentro da mesma classe. O N15S apresentou estrutura, estabilidade e localização celular levemente anormal. O P23H mostrou-se altamente anormal, tanto em nível molecular quanto celular. II) A ligação de pequenas moléculas à rodopsina foi investigada, utilizando-se os íons metálicos $\mathrm{Zn}^{2+}, \mathrm{Cu}^{2+}, \mathrm{Fe}^{2+}, \mathrm{Ni}^{2+}, \mathrm{Mg}^{2+}$ e $\mathrm{Mn}^{2+}$, a antocianina cianidina-3-O-glicosídeo $(\mathrm{C} 3 \mathrm{G})$ e o derivado da clorofila clorina e6 (Ce6). Verificou-se que estes ligantes interagem diretamente com a rodopsina in vitro. Evidências biofísicas indicaram efeitos diferenciados destes ligantes na função, estrutura e dinâmica da rodopsina. Entre todos, Ce6 conferiu maior estabilidade à rodopsina in vitro. III) A avaliação dos efeitos do Ce6 na estabilização da rodopsina foi testada in vivo: (a) Primeiramente, ratos Sprague Dawley (SD) normais foram submetidos à degeneração por luz. O tratamento com Ce6 apresentou um efeito muito pequeno na prevenção da degeneração retiniana induzida por 
fototoxicidade. (b) Em seguida, o efeito do Ce6 na progressão da RP foi avaliado nos ratos modelos P23H e Ser334ter (S334ter). Análises histológicas e funcionais indicaram que o Ce6 parece exercer um efeito positivo diminuindo a taxa de degeneração dos fotorreceptores dos ratos $\mathrm{P} 23 \mathrm{H}$. Por outro lado, o Ce6 aumentou a degeneração dos fotorreceptores do rato S334ter. Coletivamente, os estudos apresentados aumentam o conhecimento relacionado às diversas mutações da RP, P23H, N15S e S334ter, as quais causam a má-formação da rodopsina in vitro e/ou medeiam a RP in vivo. Este estudo também conduz a novas possibilidades de tratamento da RP por meio da identificação de moléculas (retinóides, íons metálicos divalentes, porfirinas e antocianinas) que podem ajudar na estabilização e formação da rodopsina, assim como na modulação da estrutura e função do fotorreceptor, objetivando a seleção específica do receptor e auxiliando diretamente em estratégias para o resgate da visão comprometida pela RP.

Palavras-chave: Retinose Pigmentar. Rodopsina. Fotorreceptores. 


\section{LIST OF FIGURES}

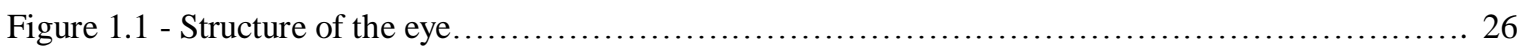

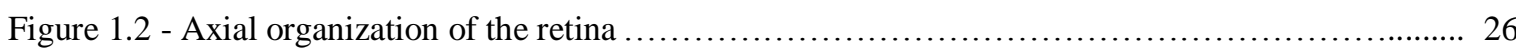

Figure 1.3 - Light micrograph of a vertical section through the human retina and a scheme identifying the

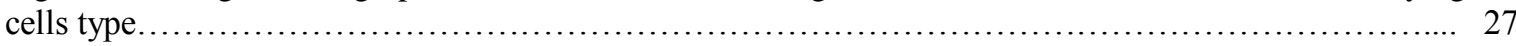

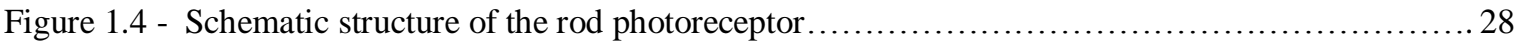

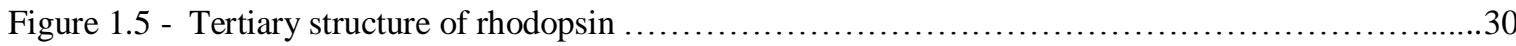

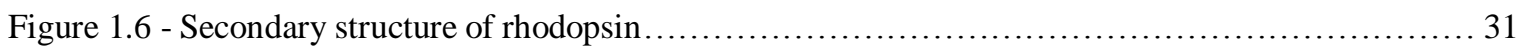

Figure 1.7 - Cartoon representation of dark state rhodopsin highlighting the presence of $\mathrm{Zn}^{2+}$ bound, PDB

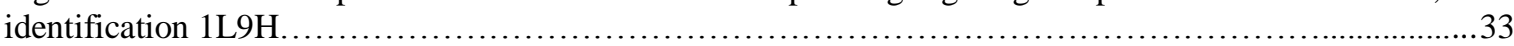

Figure 1.8 - Application of different spectroscopic methods to the analysis of rhodopsin ...................34

Figure 1.9 - Chemical structures of $\mathrm{pH}$ dependent equilibrium species of the cyanidin-3-glucoside.........35

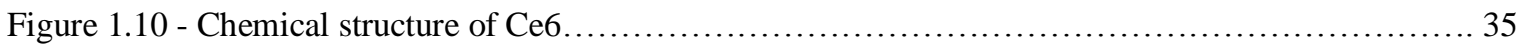

Figure 1.11 - Secondary structure model of rhodopsin showing the positions of the mutations that cause RP

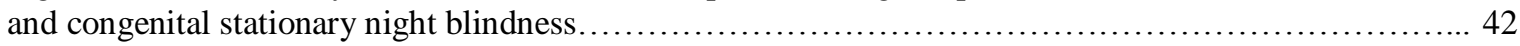

Figure 1.12 - Schematic representation of the initiation of the retinal degeneration in each animal model 46

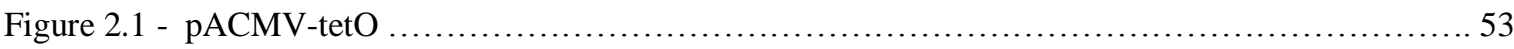

Figure 2.2 - Activation of sepharose by $\mathrm{CNBr}$ and coupling of protein to the activated gel............... 58

Figure 2.3 - Schematic diagram of a glass slide containing the retinal sections........................ 63

Figure 2.4 - Structure of the eye, showing how the retinal thickness measurements were performed....... 73

Figure 3.1 - Secondary structure model of rhodopsin ................................................ 79

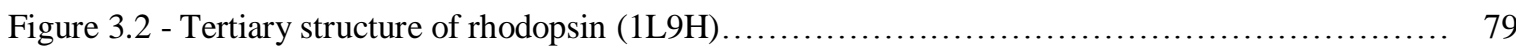

Figure 3.3 - Purification profile of WT rhodopsin (Blue) and mutants P23H (Red) and N15S (Green).. 81

Figure 3.4 - Rhodopsin stability measured by loss of $500 \mathrm{~nm}$ chromophore over time at $55{ }^{\circ} \mathrm{C} \ldots \ldots \ldots \ldots . \quad 82$

Figure 3.5 - Rhodopsin stability measured by loss of $500 \mathrm{~nm}$ chromophore over time at $37^{\circ} \mathrm{C} \ldots \ldots \ldots \ldots . \quad 84$

Figure 3.6 - Western-blot of WT and rhodopsin mutants (N15S and P23H) expressed transiently in COS-1

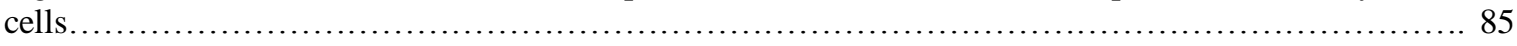

Figure 3.7 - Localization of WT and mutant rhodopsin............................................. 87

Figure 3.8 - The ATR / FTIR amide I band region along with the de-convoluted spectra corresponding to the different secondary structure fractions of (A) WT, (B) N15S and (C) P23H mutants of rhodopsin in $\mathrm{H}_{2} 0$ and

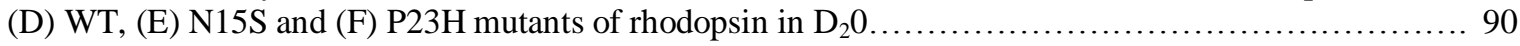

Figure 3.9 - Rhodopsin meta-II decay monitored by the use of fluorescence spectroscopy............. 91

Figure 4.1 - Thermal denaturation studies of rhodopsin in the presence of Chlorin e6 (Ce6) using circular

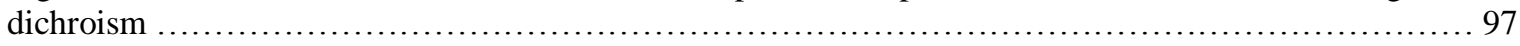

Figure 4.2 - Thermal melting curves of rhodopsin..................................................... 98 
Figure 4.3 - Effects of Chlorin e6 (Ce6) on thermal stability of rhodopsin at $55{ }^{\circ} \mathrm{C}$ using fluorescence

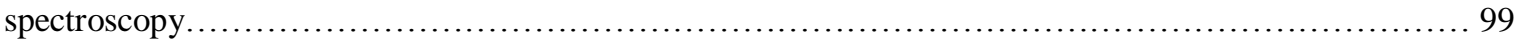

Figure 4.4 - Effects on rhodopsin Meta II determined by fluorescence spectroscopy..................100

Figure 4.5 - Thermal denaturation studies of rhodopsin in the presence of different metal ions and Ce6 using

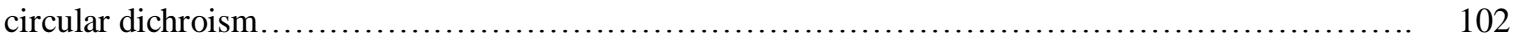

Figure 4.6 - Thermal melting curves of rhodopsin...................................... 105

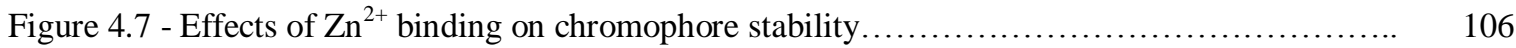

Figure 4.8 - Thermal denaturation measured using fluorescence spectroscopy.................. 108

Figure 4.9 - Effects of Chlorin e6 $(\mathrm{Ce} 6)$ and $\mathrm{Zn}^{2+}$ on thermal stability of rhodopsin at $55{ }^{\circ} \mathrm{C}$ using

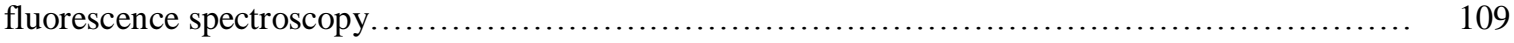

Figure 4.10 - Effect of metal ions on rhodopsin Meta II decay monitored by fluorescence spectroscopy 111

Figure 4.11 - The mean residue ellipticity at $222 \mathrm{~nm}$ of rhodopsin $(1.5 \mu \mathrm{M})$ at $\mathrm{pH} 4,6$ and 8 as a function of temperature in the (A) absence and (B) presence of a 10-fold excess of C3G (15 $\mu \mathrm{M})$ over rhodopsin 113

Figure 4.12 - Binding of $\mathrm{C} 3 \mathrm{G}$ to rhodopsin in the dark and in the light as monitored by fluorescence spectroscopy.

Figure 4.13 - Fluorescence traces of thermal denaturation of rhodopsin in the absence (black lines) and presence (red lines) of $\mathrm{C} 3 \mathrm{G}$ at (A) $\mathrm{pH} 4$, (B) $\mathrm{pH} 6$ and (C) $\mathrm{pH} 8$....

Figure 4.14 - Stability of rhodopsin $(0.5 \mu \mathrm{M})$ in the absence (black lines) and presence of $5 \mu \mathrm{M}$ C3G (red lines) at $55{ }^{\circ} \mathrm{C}$ measured by fluorescence spectroscopy at (A) $\mathrm{pH} 4$, (B) $\mathrm{pH} 6$ and (C) $\mathrm{pH} 8 \ldots \ldots \ldots$.

Figure 4.15 - Effects of $\mathrm{C} 3 \mathrm{G}$ binding on chromophore stability at $50{ }^{\circ} \mathrm{C}$ monitored by UV/Visible Spectroscopy, (A) at $\mathrm{pH} 4,(\mathrm{~B})$ at $\mathrm{pH} 6$, and (C) at $\mathrm{pH} 8$.

Figure 5.1 - Apoptotic photoreceptors after light-induced retinal degeneration.

Figure 5.2 - Comparison of normal and light-induced degeneration, and TUNEL

Figure 5.3 - DMTU protects the retina against light-induced degeneration.

Figure 5.4 - Effects of Ce6 in light-induced retinal degeneration

Figure 6.1 - Rates of degeneration in mutant rhodopsin transgenic rats.

Figure 6.2 - Retinal degeneration in the mutant rhodopsin transgenic rat $\mathrm{P} 23 \mathrm{H}$.

Figure 6.3 - Retinal degeneration in the mutant rhodopsin transgenic rat S334ter....

Figure 6.4 - Diagram illustrating the 5 basic ERG responses defined by the ISCEV (1999 update)....

Figure 6.5 - Example of ERG parameters that are normally measured in the ophthalmic clinic for electrodiagnosis.

Figure 6.6 - Retinal sections of P23H rats treated with PBS $(n=2)$, different concentrations of Ce6 $(2,10$ and $20 \mathrm{mg} / \mathrm{Kg})(\mathrm{n}=2$ for each concentration $)$ and DMTU $(500 \mathrm{mg} / \mathrm{Kg})(\mathrm{n}=2)$.

Figure 6.7 - Comparison of $\mathrm{P} 23 \mathrm{H}$ rats $\mathrm{Ce} 6$ treated and non-treated at $\mathrm{P} 60, \mathrm{P} 90$ and $\mathrm{P} 150$

Figure 6.8 - Mean ONL thickness of the Ce6 treated and non-treated P23H rats.

Figure 6.9 - Measurements of ONL thickness plotted as a function of the distance from the optical nerve head. 
Figure 6.10 - Cross sections of the retina showing immunofluorescence for anti-PKC alpha, a marker for rod bipolar cells in non-treated (A-C) and Ce6 treated (D-F)

Figure 6.11 - Cross sections of the retina showing immunofluorescence for anti-PKC alpha, a marker for rod bipolar cells in non-treated (left column) and Ce6 treated (right column) at P90................. 142

Figure 6.12 - Rhodopsin immunolabeling in non-treated P23H rats......................... 144

Figure 6.13 - Example of one rat response of ERGs total field (signal in $\mu \mathrm{V}$ ) of non-treated versus Ce6 treat

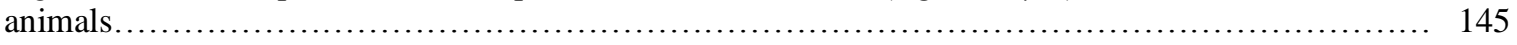

Figure 6.14 - ERG a-wave responses of P23H rats...................................... 146

Figure 6.15 - Average of a-wave amplitude (maximum scotopic response - $30 \mathrm{~cd} \mathrm{~s} / \mathrm{m}^{2}$ )........... 147

Figure 6.16 - Average of a-wave latency (maximum scotopic response $-30 \mathrm{~cd} \mathrm{~s} / \mathrm{m}^{2}$ )............ 148

Figure 6.17 - ERG amplitude response to different filters in P23H rat........................ 149

Figure 6.18 - ERG amplitude response in $\mathrm{P} 23 \mathrm{H}$ rat....................................... 150

Figure 6.19 - ERG latency response in $\mathrm{P} 23 \mathrm{H}$ rat........................................ 151

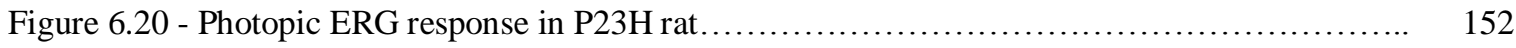

Figure 6.21 - Example of Flicker ERG responses at different ages......................... 153

Figure 6.22 - Average of $1^{\text {st }}$ harmonic response as a function of age at 12 and $30 \mathrm{~Hz}$ temporal frequencies................................................................... 154

Figure 6.23 - Comparison of S334ter rats treated and non-treated with Ce6 at P60, P90 and P150... 155

Figure 6.24 - Mean ONL thickness of the Ce6 treated and non-treated S334ter rats................ 156

Figure 6.25 - Measurements of ONL thickness plotted as a function of the distance from the optical nerve

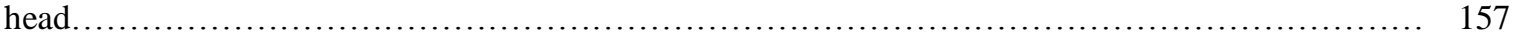

Figure 6.26 - Example of one rat response of ERGs total field (signal in $\mu \mathrm{V}$ ) of non-treated versus Ce6 treat

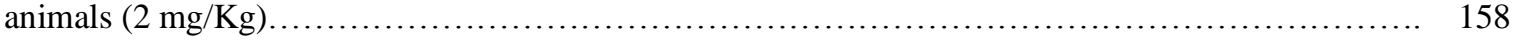

Figure 6.27 - ERG amplitude response to different filters in S334ter rat....................... 159

Figure 6.28 - Average of a-wave amplitude (maximum scotopic response - $30 \mathrm{~cd} . \mathrm{s} / \mathrm{m}^{2}$ ).......... 160

Figure 6.29 - Average of a-wave latency (maximum scotopic response - 30cd s $/ \mathrm{m}^{2}$ )............. 161

Figure 6.30 - ERG amplitude response as function of different filters in S334ter rats............. 162

Figure 6.31 - ERG amplitude response in S334ter rats as a function of age..................... 163

Figure 6.32 - ERG latency of b-wave response in S334ter rats............................ 164

Figure 6.33 - Photopic ERG response in S334ter rat.................................... 165

Figure 6.34 - Example of Flicker ERG responses at different ages......................... 166

Figure 6.35 - Average of $1^{\text {st }}$ and $3^{\text {rd }}$ harmonic response as function of age at 12 and $30 \mathrm{~Hz}$ temporal

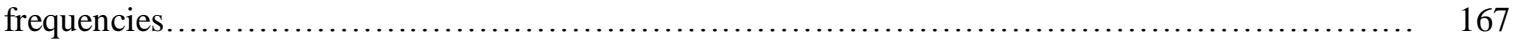

Figure 7.1 - Pedigrees of the two families investigated in this study..................... 177

Figure 7.2 - Representative ISCEV-standard ERG response................................ 178

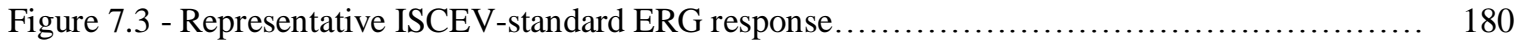


Figure 7.4 - Schematic representation of the in vitro and in vivo experimental steps taken in this work 182

Figure 7.5 - Suggested concentration areas for future work.............................. 183 


\section{LIST OF TABLES}

Table 3.1 - The table shows the calculated secondary structure content of the WT and the mutants N15S and $\mathrm{P} 23 \mathrm{H}$ before and after H/D exchange from the ATR-FTIR spectra recorded in the amide I region

Table 4.1 - Meta II decay half-lives of rhodopsin in the absence and presence of Ce6 at various concentrations indicated.

Table 4.2 - Percent decrease in mean residue ellipticity at $222 \mathrm{~nm}$ at $100{ }^{\circ} \mathrm{C}$, minimum $\alpha$-helix content present at $100{ }^{\circ} \mathrm{C}$ after thermal denaturation, and midpoint transition temperatures of rhodopsin in the presence of different metal ions and $\mathrm{Ce} 6$.

Table 4.3 - The half-lives and Meta II decay rates of rhodopsin in the absence and in the presence of metal ions. 


\section{LIST OF ABBREVIATIONS}

\begin{tabular}{|c|c|}
\hline${ }^{15} \mathrm{~N}$ & nitrogen-15 \\
\hline${ }^{19} \mathrm{~F}$ & fluorine-19 \\
\hline${ }^{1} \mathrm{H}$ & proton-1 \\
\hline $\mathrm{A}_{500}$ & absorbance (at $500 \mathrm{~nm}$ ) \\
\hline $\mathrm{AD}$ & autosomal dominat \\
\hline ADRP & autosomal dominat retinitis pigmentosa \\
\hline AMD & age-related macular degeneration \\
\hline APS & ammonium persulfate \\
\hline AR & autosomal recessive \\
\hline ARRP & autosomal recessive retinitis pigmentosa \\
\hline ATR/FT-IR & Atenuated Total Reflectance Fourier Transform Infrared \\
\hline BBS & Bardet Biedl syndrome \\
\hline BDNF & brain-derived neurotrophic factor \\
\hline BS & bovine serum \\
\hline BSA & bovine serum albumin \\
\hline bFGF & basic fibroblast growth factor \\
\hline $\mathrm{C} 3 \mathrm{G}$ & cyanidin-3-glucoside \\
\hline $\mathrm{CD}$ & circular dichroism \\
\hline Ce6 & chlorin e6 \\
\hline cGMP & cyclic GMP \\
\hline CNTF & neurotrophic factor \\
\hline COS, COS-1 & $\begin{array}{l}\text { cells derived from african green monkey, CV-1 (simian) in origin, and } \\
\text { carrying the SV40 genetic material }\end{array}$ \\
\hline $\mathrm{CP}$ & cytoplasmic \\
\hline CSL & cone sensitivity loss \\
\hline $\mathrm{D}_{2} \mathrm{O}$ & deuterium oxide, heavy water \\
\hline DAPI & 4',6-diamidino-2-phenylindole \\
\hline DM & dodecyl maltoside \\
\hline DMEM & Dulbecco's modified Eagle's medium high glucose \\
\hline DMSO & Dimethyl sulfoxide \\
\hline DMTU & dimethylthiourea \\
\hline $\mathrm{EC}$ & extracellular \\
\hline ER & endoplasmic reticulum \\
\hline ERG & electroretinogram \\
\hline FBS & fetal bovine serum \\
\hline GCL & ganglionar cell layer \\
\hline GDNF & glial cell-line derived neurotrophic factor \\
\hline GDP & guanosine 5'-diphosphate \\
\hline GMP & guanosine monophosphate \\
\hline GPCR & G-protein coupled receptor \\
\hline Gt & G-protein transducin \\
\hline GTP & guanosine 5'-triphosphate \\
\hline
\end{tabular}




\begin{tabular}{|c|c|}
\hline GVFs & Goldmann visual fields \\
\hline $\mathrm{H} / \mathrm{D}$ & hydrogen/deuterium \\
\hline HEK293 & Human embryonic kidney 293 cells \\
\hline INL & inner nuclear layer \\
\hline IPL & inner plexiform layer \\
\hline IRE & internal reflection element \\
\hline ISCEV & International Society for Clinical Electrophysiology of Vision \\
\hline LCA & Leber's congenital amaurosis \\
\hline MCT & mercury cadmium telluride \\
\hline Meta II & metarhodopsin II \\
\hline Napi & sodium phosphate buffer \\
\hline NDS & normal donkey serum \\
\hline NEB & Quick ligase enzyme \\
\hline NMR & nuclear magnetic resonance \\
\hline OCT & optimum cutting temperature \\
\hline OFL & optic fiber layer \\
\hline OG & octyl glucoside \\
\hline OGT & ocular gene therapy \\
\hline ONL & outer nuclear layer \\
\hline OPL & outer plexiform layer \\
\hline $\mathrm{P}$ & postnatal day \\
\hline PBN & phenyl-N-tert-butylnitrone \\
\hline PBS & phosphate buffer saline \\
\hline PDB ID & protein data bank identification code \\
\hline PDE & phosphodiesterase \\
\hline pMT4 & plasmid-DNA \\
\hline PNGase F & Peptide: N-Glycosidase F \\
\hline PRA & progressive retinal atrophy \\
\hline PVDF & polyvinylidene difluoride \\
\hline $\mathrm{RHO}$ & rhodopsin gene \\
\hline ROS & rod outer segment \\
\hline $\mathrm{RP}$ & retinitis pigmentosa \\
\hline RPGR & RP GTPase regulator \\
\hline RSL & rod sensitivity loss \\
\hline SCA7 & autosomal dominant cerebellar ataxia type II \\
\hline SD & sprague-dawley \\
\hline SDS & sodium dodecyl sulfate \\
\hline SDS-PAGE & sodium dodecyl sulfate polyacrylamide gel electrophoresis \\
\hline SLS & Senior Loken syndrome \\
\hline$t_{1 / 2}$ & half life \\
\hline TEMED & tetramethylethylenediamine \\
\hline TM & transmembrane \\
\hline TUDCA & tauroursodeoxycholic acid \\
\hline UV/VIS & ultraviololet/visible \\
\hline
\end{tabular}


wild-type

XL

$\mathrm{X}$-linked recessive

Residues at particular positions in rhodopsin are designated by using the three-letter code for the amino acid, followed by its sequence number. 


\section{TABLE OF CONTENTS}

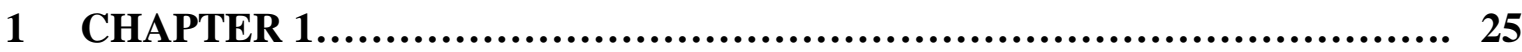

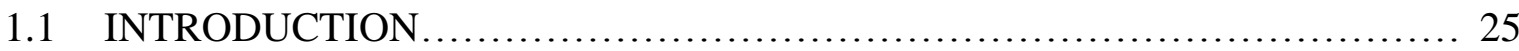

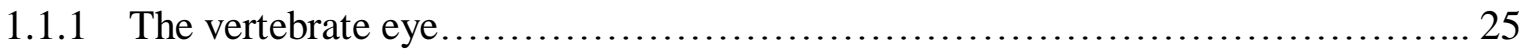

1.1 .2 Rhodopsin........................................................... 29

1.1.3 Allosteric ligands for rhodopsin........................................... 32

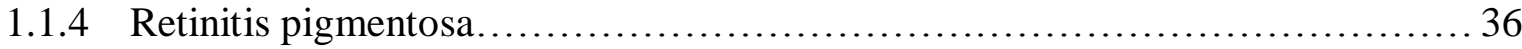

1.1.5 Types of Retinitis pigmentosa......................................... 37

1.1.6 Current therapies............................................................. 39

1.1.7 Rhodopsin as a target in retinitis pigmentosa............................ 41

1.1.8 Animal models of retinitis pigmentosa....................................42

1.2 OPEN QUESTIONS, GOALS AND ACCOMPLISHMENTS ................. 47

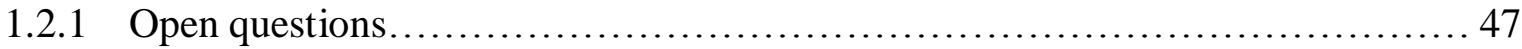

1.2.2 Objectives and Approaches.............................................. 48

2 CHAPTER 2: EXPERIMENTAL PROCEDURES ........................... 49

2.1 EXPRESSION AND PURIFICATION OF RHODOPSIN ...................... 49

2.1.1 Preparation of rhodopsin from bovine retinae................................49

2.1.2 Expression in COS-1 cells............................................ 50

2.1.2.1 Construction of rhodopsin mutant genes in pMT4 for transient transfection ... 50

2.1.2.2 COS-1 cell culture and transient transfection ....................................... 50

2.1.3 Expression of rhodopsin in HEK293 cells.............................. 52

2.1.3.1 Construction of rhodopsin expression plasmid using pACMV-tetO ................52

2.1.3.2 Expression of HEK293 stable cell lines containing the opsin mutant genes....... 54

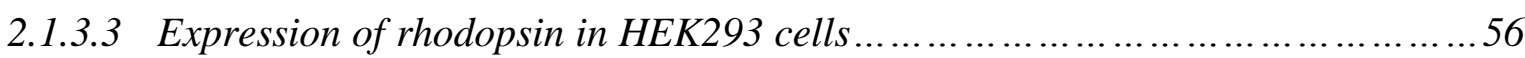

2.1.4 Reconstitution of the opsin expressed in mammalian cells with 11-cis retinal......56

2.1.5 Reconstitution of the opsin expressed in mammalian cells with 9-cis retinal......57

2.1.6 Reconstitution of opsin during synthesis in HEK293 cells with 9-cis and 11-cis

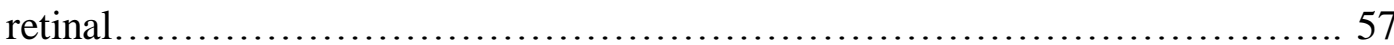

2.1.7 Solubilization of cell pellets containing rhodopsin using detergents............. 57

2.1.8 Affinity chromatography of rhodopsin using 1D4-sepharose................. 58

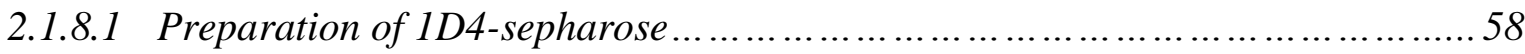

2.1.8.2 Binding of solubilized rhodopsin to 1D4-sepharose ............................... 60

2.1.8.3 Elution of rhodopsin from 1D4-sepharose ......................................61 
2.2.1 Breeding and maintenance of wild-type, homozygous $\mathrm{P} 23 \mathrm{H}$ and $\mathrm{S} 334$ ter rats.... 61

2.2.2 Light-induced degeneration after treatment with DMTU and chlorine e6 (Ce6) ...62

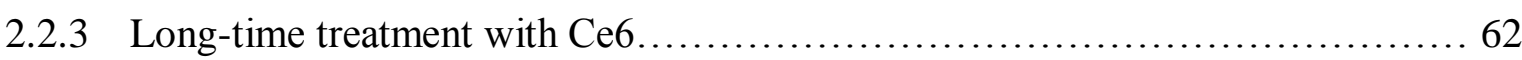

2.2.4 Extraction and fixation of rat eyes....................................... 63

2.2.5 Sectioning, storage and processing of rat eyes............................. 63

2.3 SPECTROSCOPIC AND MICROSCOPIC TECHNIQUES ...................... 64

2.3.1 Absorbance Spectroscopy............................................. 64

2.3.2 Fluorescence Spectroscopy......................................... 65

2.3.3 Circular Dichroism Spectroscopy.....................................65

2.3.4 Total Reflectance Fourier Transform Infrared (ATR/FT-IR) spectroscopy........66

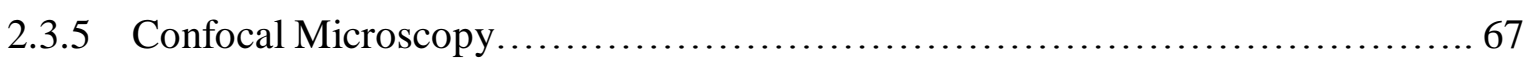

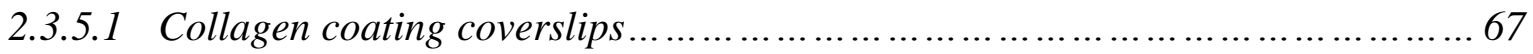

2.3.5.2 Growing and prepararing cells on coverslips for imaging ..................... 67

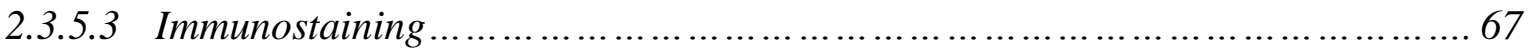

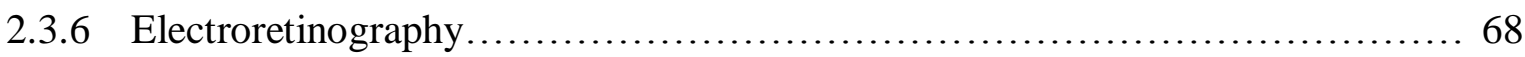

2.3.7 Fluorescence Microscopy.............................................. 69

2.4 ANALYTICAL PROCEDURES ........................................ 70

2.4.1 Protein quantification................................................ 70

2.4.2 Meta II decay..................................................... 70

2.4.3 Binding of small molecules monitored by fluorescence spectroscopy............ 71

2.4.4 Thermal denaturation................................................... 71

2.4.5 Deglycosylation using PNGase-F....................................... 72

2.4.6 Retinal Layer Thickness ................................................ 72

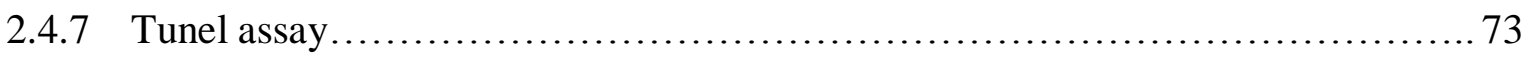

2.4.8 SDS PAGE Analysis.............................................. 74

2.4.9 Western Blot....................................................... 75

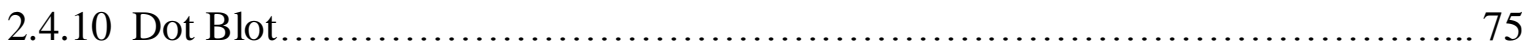

3 CHAPTER 3: MOLECULAR CHARACTERIZATON OF WT, N15S AND P23H MUTANTS OF RHODOPSIN IN MAMMALIAN CELLS AND THEIR INTERACTION WITH CE6............................................. 77

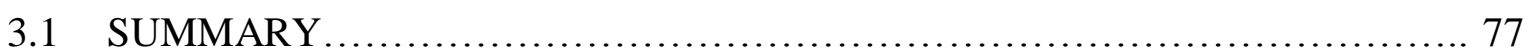

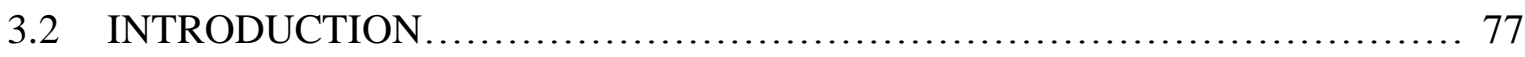

3.2.1 Expression and purification of mutant and wt rhodopsin..................... 81 
3.2.1.1 Transient transfection of WT, N15S and P23H mutants of rhodopsin...............81

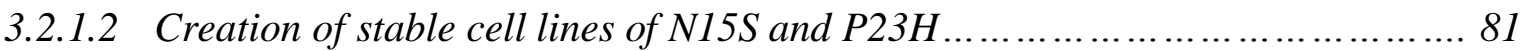

3.2.1.3 Reconstitution of mutant and WT rhodopsin with 9-or 11-cis retinal.............. 82

3.2.1.4 Addition of 9-or 11-cis retinal to cells during synthesis ........................... 83

3.2.2 Molecular characterization of WT, N15S and PS23H rhodopsin................ 84

3.2.2.1 Glycosylation and aggregation of WT, N15S and P23H in COS-1 cells...........84

3.2.2.2 Intracellular localization studies using confocal microscopy ...................... 86

3.2.2.3 Rescue of chromophore in N15S and P23H mutants by the addition of retinal...87

3.2.2.4 Structural characterization using FTIR spectroscopy analysis of WT, N15S and

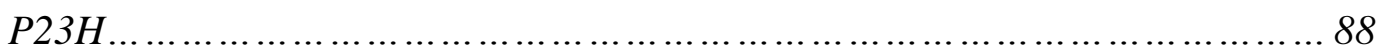

3.2.2.5 Comparison of Meta II decay rates.................................................. 91

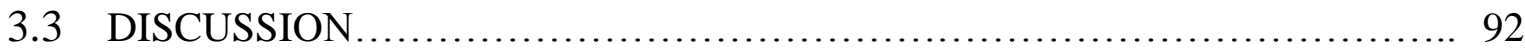

3.4 CONCLUSIONS ....................................................... 93

4 CHAPTER 4: SMALL MOLECULE BINDING TO WT RHODOPSIN PURIFIED FROM BOVINE RETINAE....................................... 94

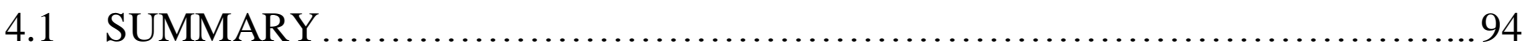

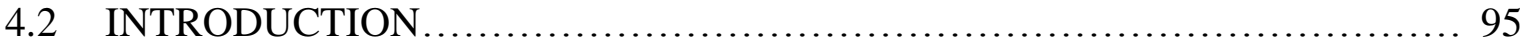

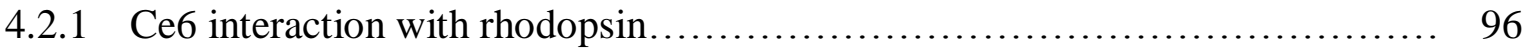

4.2.1.1 Effects of Ce6 binding on secondary structure of rhodopsin .......................96

4.2.1.2 Effects of Ce6 binding on tertiary structure of rhodopsin ...........................97

4.2.1.3 Effect of Ce6 binding on the Meta II decay of rhodopsin ..............................99

4.2.2 Metal ions effects on rhodopsin in the presence and absence of Ce6........... 101

4.2.2.1 Effects of Ce6 and metal ion binding on secondary structure of rhodopsin......101

4.2.2.2 Effects of Ce6 binding on tertiary structure of rhodopsin........................ 106

4.2.2.3 Effects of Ce6 and bivalent metal ions binding on the Meta II decay............. 109

4.2.3 Cyanidin-3-Glucoside (C3G) interaction with rhodopsin................... 112

4.2.3.1 Effects of C3G binding on secondary structure of rhodopsin .................... 112

4.2.3.2 Effects of C3G binding on tertiary structure of rhodopsin ....................... 113

4.2.3.3 pH dependent alteration of structure and stability of rhodopsin in the absence

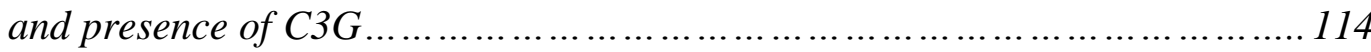

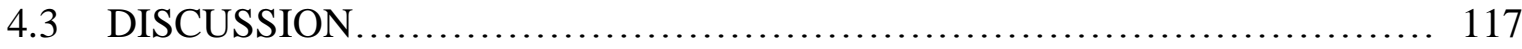

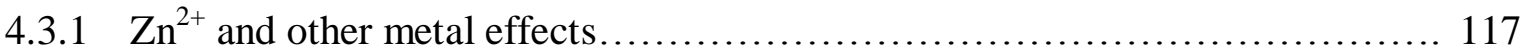

4.3.2 Ce6 effects with and without bivalent metal ions........................... 119

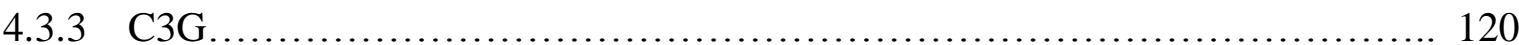


4.4 CONCLUSIONS.

5 CHAPTER 5: DRUG TREATMENT OF LIGHT-DAMAGED SPRAGUEDAWLE (SD) RATS IN VIVO ............................................. 122

5.1 SUMMARY ......................................................... 122

5.2 INTRODUCTION ................................................. 122

5.2.1 Protection of rat retina from light induced degeneration with different drugs.... 123

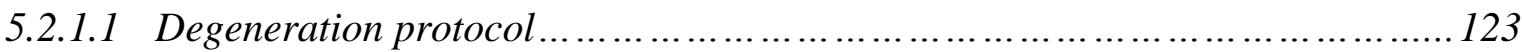

5.2.1.2 DMTU effect on light-induced apoptosis of SD rat retina ........................ 126

5.2.1.3 Ce6 effect on light-induced apoptosis of SD rat retina ............................. 127

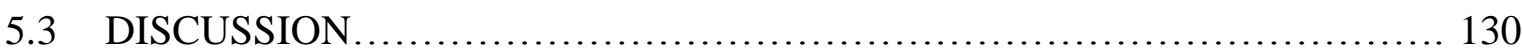

6 CHAPTER 6: CE6 TREATMENT OF P23H AND S334TER MUTANT RATS

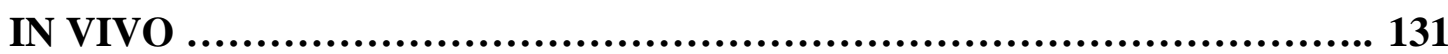

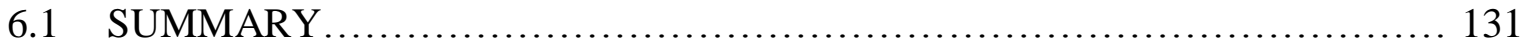

6.2 INTRODUCTION ...................................................... 131

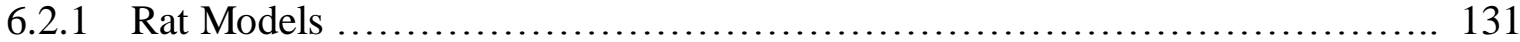

6.2.2 ERG

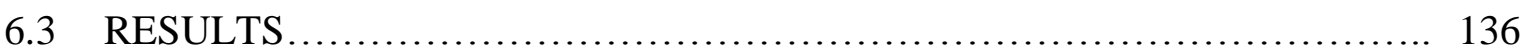

6.3.1 Treatment of $\mathrm{P} 23 \mathrm{H}$ rats with Ce6..................................... 136

6.3.1.1 Dose dependent effect of Ce6 on photoreceptor cell death ...................... 136

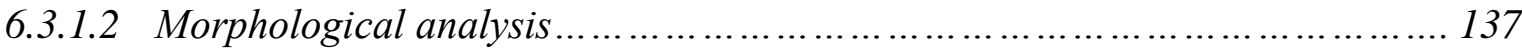

6.3.1.3 Bipolar cell morphology .................................................... 140

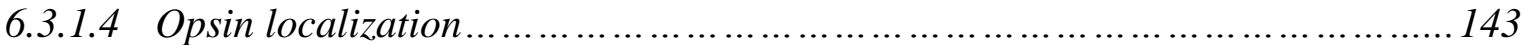

6.3.1.5 ERG analysis of $\mathrm{P} 23 \mathrm{H}$ rats after treatment with Ce6 ............................ 145

6.3.2 Treatment of S334ter rats with Ce6................................... 154

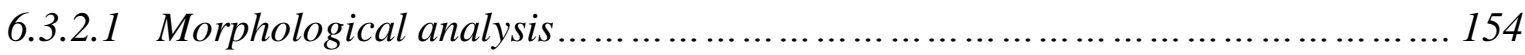

6.3.2.2 ERG analysis of S334ter rats after treatment with Ce6 ........................ 157

6.4 DISCUSSION...................................................... 167

6.5 CONCLUSIONS ..................................................... 171

7 CHAPTER 7: INTEGRATION OF IN VITRO, IN VIVO RAT MODEL AND PATIENT STUDIES IN RETINITIS PIGMENTOSA....................... 172

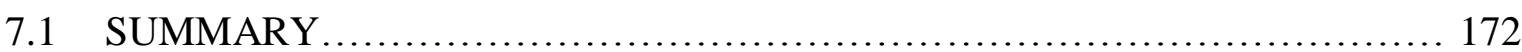

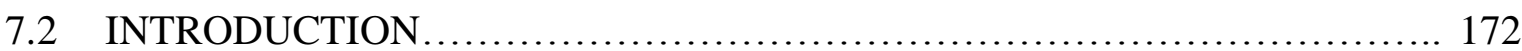

7.2.1 Summary of patient data carrying $\mathrm{P} 23 \mathrm{H}$ and N15S mutations................. 174

7.2.2 Comparison of $\mathrm{P} 23 \mathrm{H}$ and $\mathrm{N} 15 \mathrm{~S}$ human patient data with in vitro studies........ 178 
7.2.3 Comparison of $\mathrm{Ce} 6$ effects on $\mathrm{RP}$ progression in vivo versus in vitro model

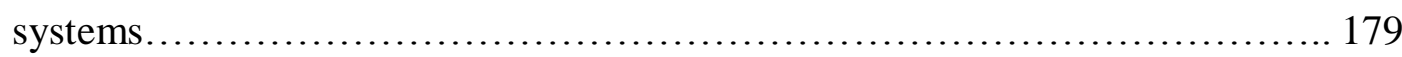

7.3 FUTURE WORK - PROSPECTS FOR NOVEL APPROACHES TO PREVENTING AND TREATING RP IN PATIENTS..................... 179

7.4 IMPACT ............................................................ 183

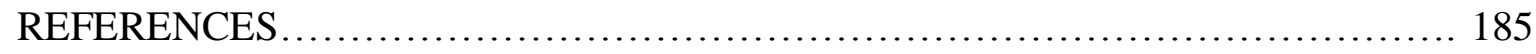




\section{CHAPTER 1}

\subsection{INTRODUCTION}

Vision is an important sensory model system for vertebrates since it most directly mediates the interaction with the exterior world. Not surprisingly, the eye has evolved to be an organ of extreme perfection and complexity. This was elegantly pointed out by Charles Darwin, who said:

\footnotetext{
To suppose that the eye, with all its inimitable contrivances for adjusting the focus to different distances, for admitting different amounts of light, and for the correction of spherical and chromatic aberration, could have been formed by natural selection seems, I freely confess, absurd in the highest possible degree. Yet reason tells me, that if numerous gradations from a perfect and complex eye to one very imperfect and simple, each grade being useful to its possessor, can be shown to exist; if further, the eye does vary ever so slightly, and the variations be inherited, which is certainly the case; and if any variation or modification in the organ be ever useful to an animal under changing conditions of life, then the difficulty of believing that a perfect and complex eye could be formed by natural selection, though insuperable by our imagination, can hardly be considered real. How a nerve comes to be sensitive to light, hardly concerns more than how life itself first originated; but I may remark that several facts make me suspect that any sensitive nerve may be rendered sensitive to light, and likewise to those coarser vibrations of the air which produce sounds (Darwin, 2006). Charles Darwin 1809-1882.
}

The "perfection" of the eye can be well illustrated by the capability of adaptation that the eye presents in front of specific needs of the organisms. Vision in humans has evolved to accommodate both, daylight and night vision. Nocturnal animals have their visual systems optimized for night activity. Deep sea animal vision is adapted to the limited radiation that penetrates their habitat.

\subsubsection{The vertebrate eye}

The retina is a very complex and layered structure that lines the back of the eye (Fig.1.1 and 1.2). It has a highly ordered anatomical organization, with a few basic classes of cells located in the outer nuclear layer (rods and cones), inner nuclear layer (bipolar, horizontal, and amacrine cells) and ganglion cell layer (ganglion cells). 
Figure 1.1 - Structure of the eye.

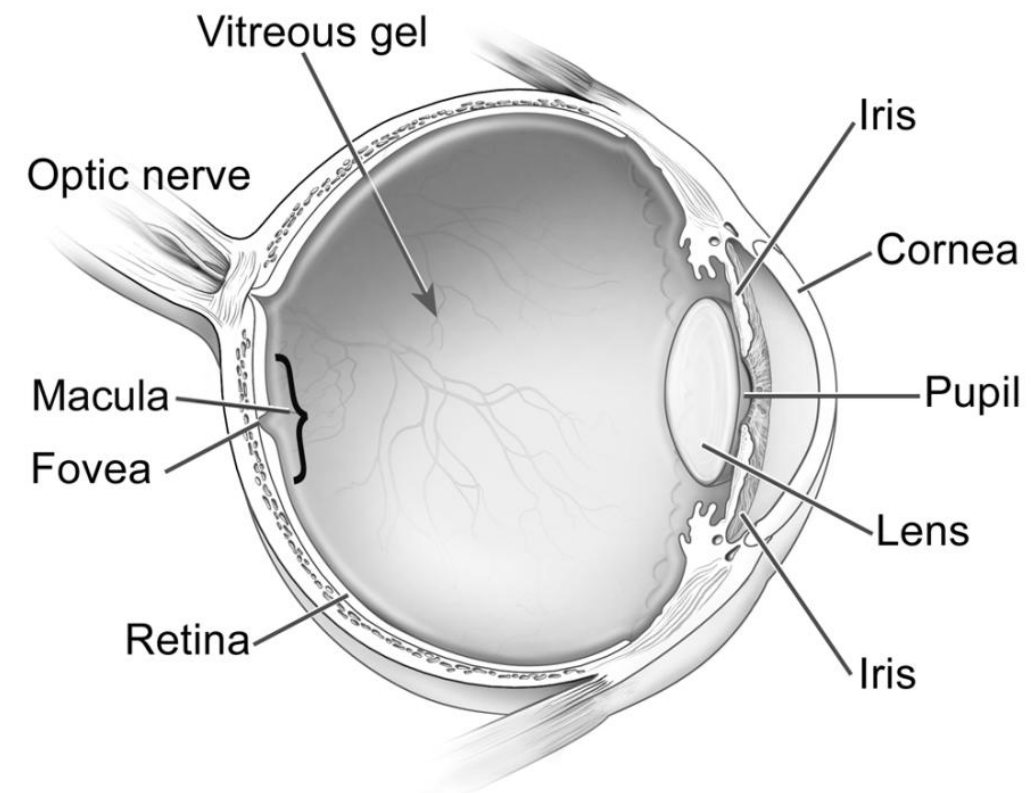

SOURCE: Eye diagram is a courtesy of the National Eye Institute, National Institutes of Health. With permission.

Figure 1.2 - Axial organization of the retina.

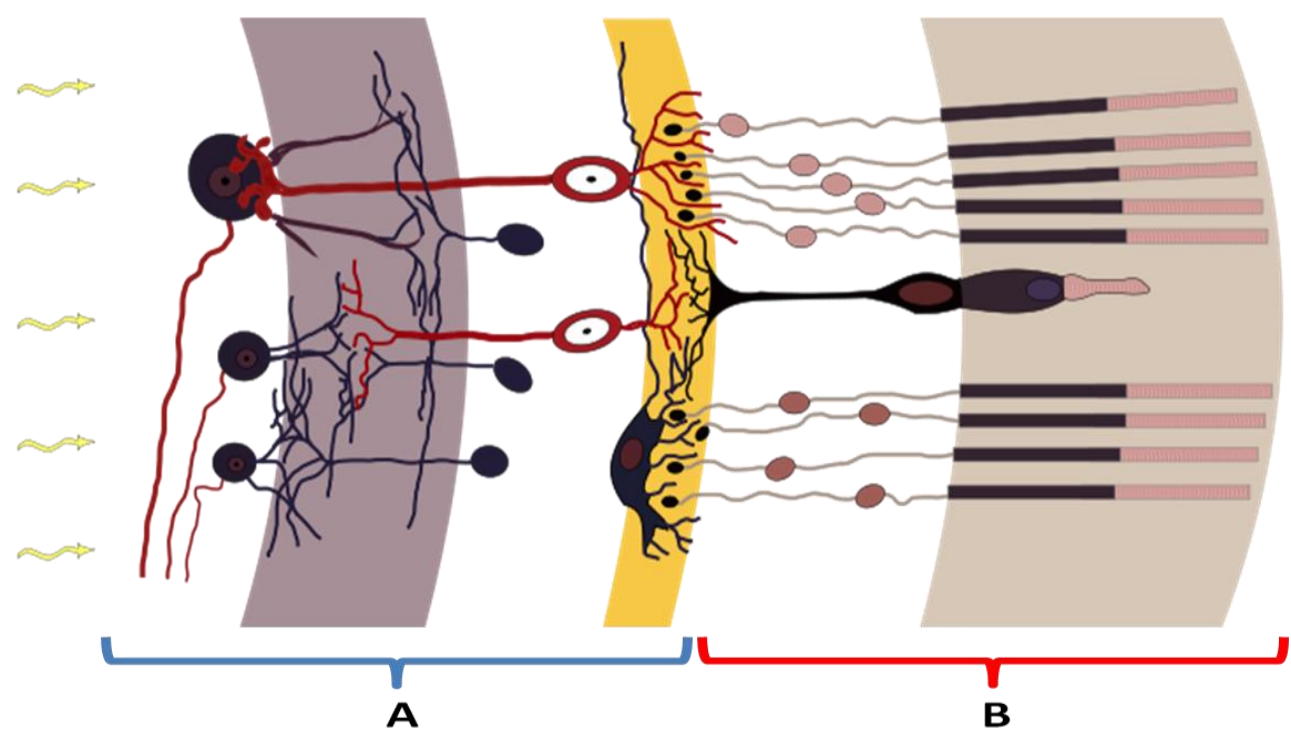

SOURCE: Drawing is adapted from (Cajal, 1900). With permission.

Figure 1.3 shows the three layers of neuronal cell bodies (outer nuclear, inner nuclear and ganglion cell layers) interconnected by synapses in the two plexiform layers (outer and inner). 
Light must pass through the entire retinal thickness to reach the outer segments of photoreceptors (rods and cones), where the phototransduction occurs. To avoid image distortion and loss, Müller glial cells, whose cell bodies are located in the inner nuclear layer, appear to act as living fiber optics (Franze et al., 2007).

Photoreceptor axons contact the dendrites of bipolar cells and horizontal cells in the outer plexiform layer (OPL). In turn, the bipolar cells transmit the signal to ganglion cell dendrites and amacrine cells in the inner plexiform layer. Last, the ganglion cells send their axons through the optic fiber layer to the optic disk to make up the optic nerve (Molavi, 1997). Photoreceptors, bipolar, and ganglion cells release glutamate to mediate the information from retina to the brain.

Figure 1.3 - Light micrograph of a vertical section through the human retina and a scheme identifying the cells type.
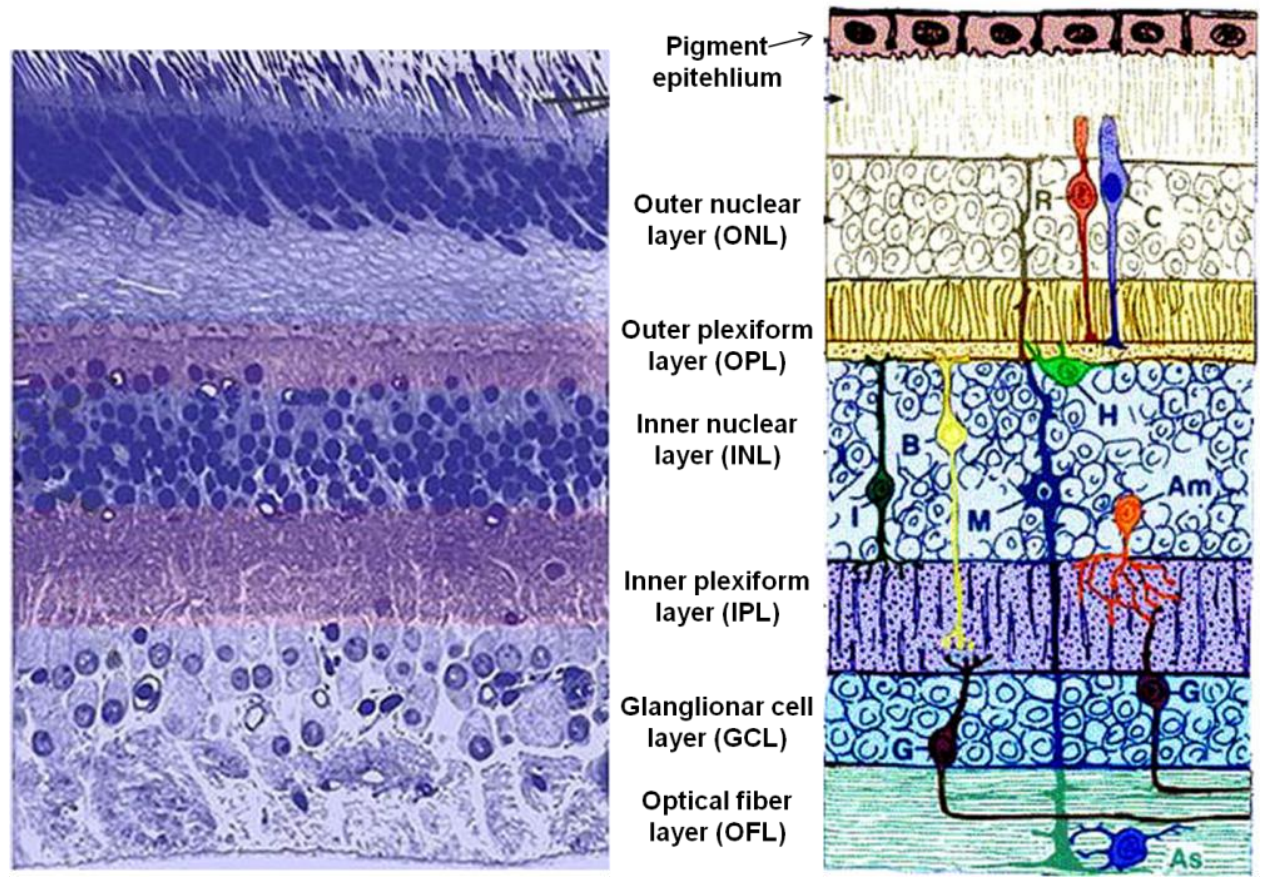

SOURCE: Figure adapted from (Kolb, 2011). With permission.

The photoreceptor cells, rods and cones, are responsible for different parts of visual perception. Rods, the most abundant photoreceptor cells, are responsible for black and white vision under dim light conditions. Rod mediated vision is very sensitive to light, but do not enable color vision. Cone mediated vision are responsible for day light vision. They present high resolution and are sensitive to color and details.

Besides rods and cones, photosensitive ganglion cells containing melanopsin were also described in mammals (Foster et al., 1991; Lucas et al., 2001; Provencio et al., 2000). They 
are involved in various reflexive responses of the brain and body to the presence of light, for example the regulation of circadian rhythms, the pupillary reflex and other non-visual responses to light.

Anatomically, vertebrate rods and cones photoreceptor cells contain an outer segment, which carries the photosensitive pigments; an inner segment, a nucleus and a synaptic terminal (Figure 1.4). The rod outer segment (ROS) is packed by a large number of discs, where the visual photoreceptor molecule rhodopsin is located. Rhodopsin consists of the apoprotein opsin and the, Vitamin A derivative, chromophore 11-cis retinal (Wald, 1968).

Figure 1.4 - Schematic structure of the rod photoreceptor.

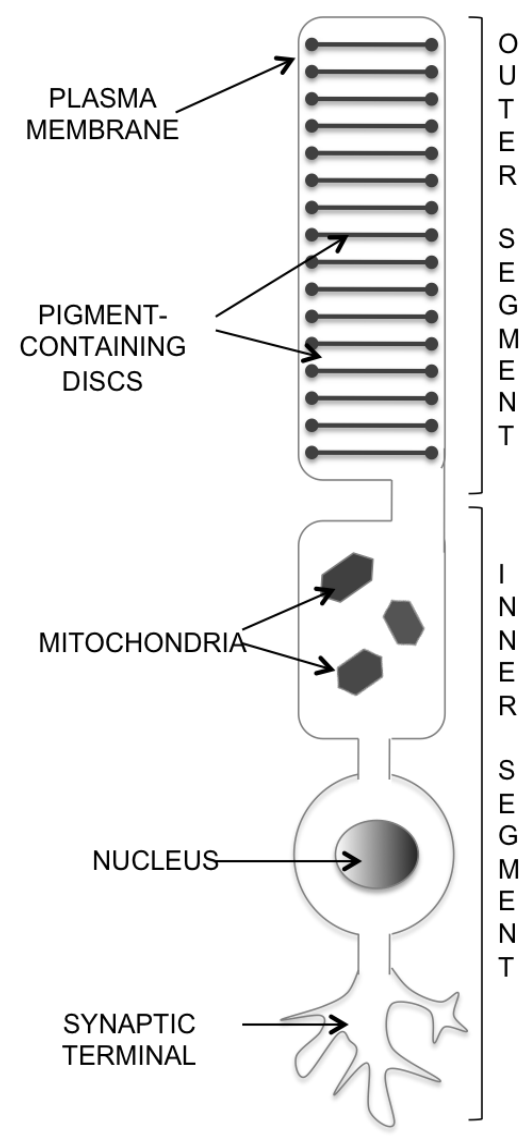

SOURCE: Balen, 2012

\subsubsection{Rhodopsin}

The photoreceptor molecule rhodopsin is a membrane protein, and a prototypical member of the large G protein-coupled receptor (GPCR) family, the largest family of cell surface receptors. Rhodopsin (Figure 1.5) is made up of a cytoplasmatic domain (CP), a 
seven-transmembrane helical domain (TM) and an extracellular domain (EC). Rhodopsin contains a covalently linked ligand, 11-cis retinal, a Vitamin A derivative, that stabilizes the folded receptor (Figure 1.5). Visual signal transduction is initiated when a photon induces isomerization of 11-cis retinal to all-trans retinal. This event triggers the rearrangement of the TM domain, resulting in the light-activated, Metarhodopsin II (Meta II) state. The Meta II state of rhodopsin is the active functionally state of the protein and initiates the visual signal transduction cascade. As a first step, Meta II binds to the G protein, transducin (Gt). Gt then activates phosphodiesterase (PDE), which hydrolyzes cyclic GMP (cGMP). This event ultimately leads to the hyperpolarization of the cell through the closure of ion channels. When the cell is hyperpolarized, the electrical potential inside the cell is more negative than in darkness. In contrast, in the dark state, cGMP keeps the channels in the photoreceptor membrane open, and $\mathrm{Na}^{+}-\mathrm{Ca}^{2+}$ influx depolarizes the membrane. The signal can be shut down through two mechanisms: (1) through Meta II decay, a process of dissociation of protein and ligand to opsin and free all-trans retinal, or (2) through phosphorylation of Meta II by rhodopsin kinase, followed by binding of arrestin to the phosphorylated C-terminal end of rhodopsin (Chabre et al., 1988). The ligand free opsin formed when Meta II decays can readily uptake 11 -cis-retinal, thus regenerating rhodopsin. 
Figure 1.5 - Tertiary structure of rhodopsin.

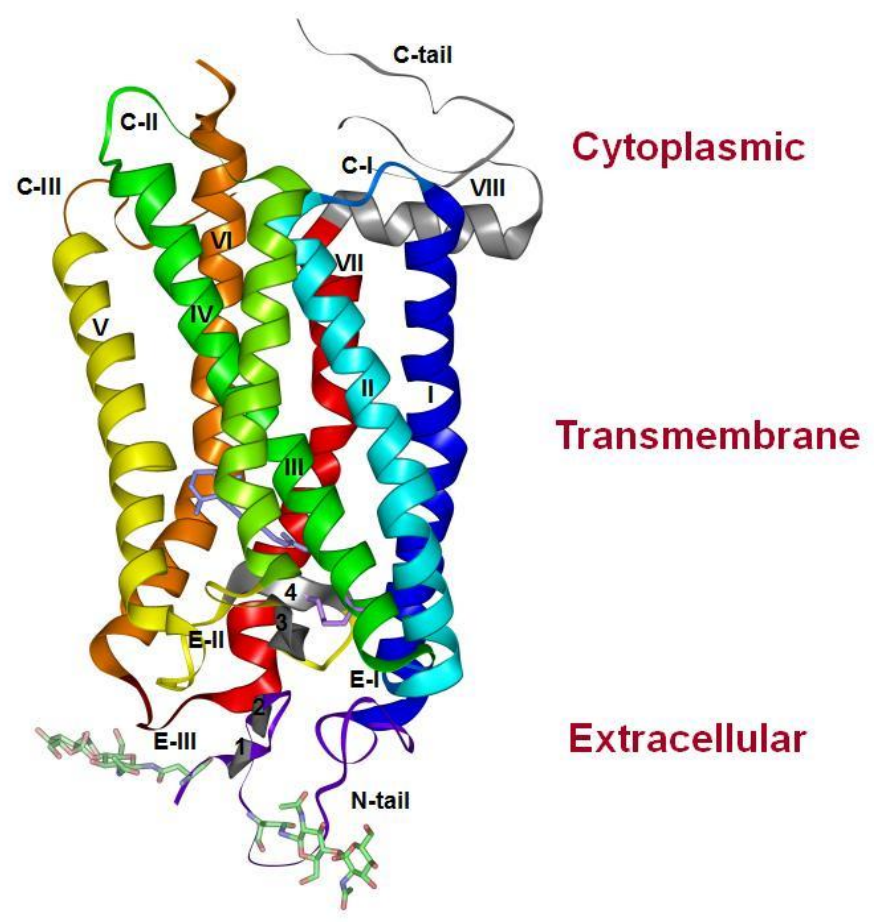

SOURCE: Balen, 2012

The primary structure of rhodopsin (bovine) contains 348 amino acids. This sequence was determined using amino acid sequencing (Hargrave et al., 1983; Ovchinnikov Yu et al., 1982). Figure 1.6 shows the secondary structure model of rhodopsin showing the seven TM helices, the EC domain and the C-terminus domain, which faces the $\mathrm{CP}$, along with the disulfide bond (Cys110 and Cys187) and palmitoylation sites (Cys322 and Cys323). The structure is divided in about 50\% TM, 25\% CP and 25\% EC domains. Rhodopsin was the first protein of the GPCR family to be crystallized (Palczewski et al., 2000). Rhodopsin it the best studied and the prototypic member of the GPCR family. 
Figure 1.6 - Secondary structure of rhodopsin.

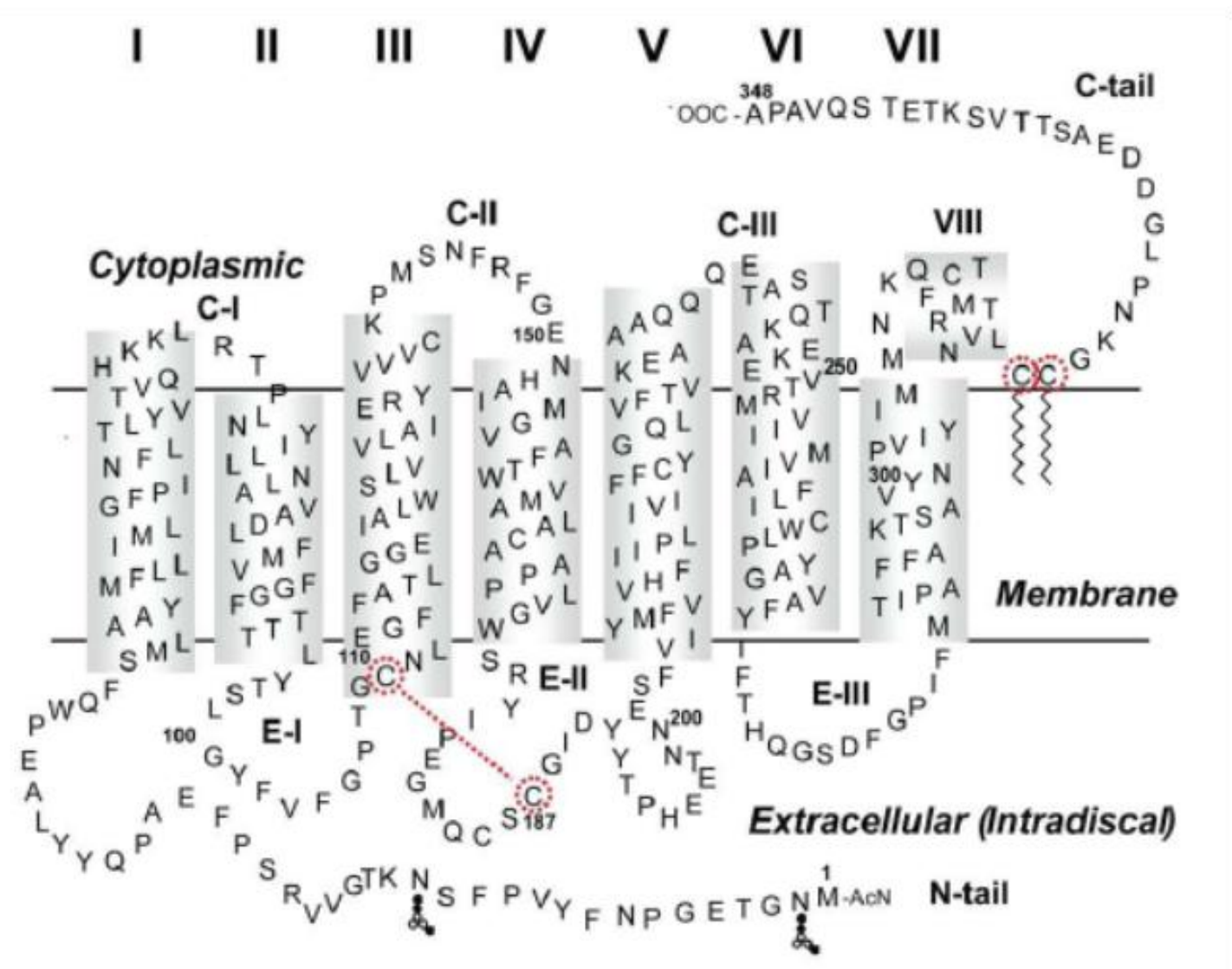

SOURCE: Balen, 2012

Since the correct folding of the rhodopsin protein is extremely important for its functionality, many studies were conducted to understand the folding and unfolding mechanisms of rhodopsin. It has been demonstrated that there is a minimum amount of helix content necessary to maintain the binding of 11-cis retinal to the protein (Ridge et al., 1995). Unfolding studies using single molecule force spectroscopic approaches, point at specific areas of rigidity in the structure of rhodopsin (Sapra et al., 2006, 2008; Tastan et al., 2007). Furthermore, in silico simulations of thermal unfolding suggested formation of a folding core between the EC and TM domains (Rader et al., 2004; Tastan et al., 2007). The structure of rhodopsin is also prone to misfolding when amino acid replacements are made (Anukanth and Khorana, 1994; Garriga et al., 1996; Kaushal and Khorana, 1994; Liu et al., 1996). Misfolding here is characterized by the inability of the protein to bind 11-cis retinal, necessary for the correct function of the protein in the visual system. 


\subsubsection{Allosteric ligands}

In addition to retinal isomers, rhodopsin has been shown to bind a number of other ligands. They all bind in locations other than the retinal binding pocket and are thus by definition allosteric ligands. These ligands are reviewed below.

\section{$\underline{\text { Zinc }}$}

Zinc, after iron, is the second most abundant trace metal in the human body (Aggett and Comerford, 1995). It is present in high concentrations in the eye, especially in the retina (Grahn et al., 2001). Numerous normal retinal functions are dependent on $\mathrm{Zn}^{2+}$, including retinal development, synaptic transmission, light response and Vitamin A metabolism (Grahn et al., 2001). $\mathrm{Zn}^{2+}$ deficiency leads to night blindness, changes in dark-adaptation, ultrastructural changes in the retina and the retinal pigment epithelium, and age-related macular degeneration (Ugarte and Osborne, 2001). Furthermore, patients with the retinal degeneration disease retinitis pigmentosa (see below) exhibit decreased serum $\mathrm{Zn}^{2+}$ levels (Karcioglu et al., 1984). However, especially for age-related macular degeneration, opposing roles have been proposed for $\mathrm{Zn}^{2+}$. While supplementation with $\mathrm{Zn}^{2+}$ is the most widely used intervention strategy for patients with this disease, its presence in Drusen, deposits of accumulated extracellular material containing misfolded proteins which especially occur in patients with age-related macular degeneration, has been linked to increased aggregation of proteins (Lengyel and Peto, 2008). There are probably multiple proteins responsible for these physiological effects (Grahn et al., 2001), but one particularly well established target is the photoreceptor rhodopsin, to which $\mathrm{Zn}^{2+}$ binds directly and specifically with milli- to micromolar affinities depending on the membrane environment studied (Shuster et al., 1992). The crystal structure of rhodopsin revealed four putative $\mathrm{Zn}^{2+}$ binding sites (Okada et al., 2002; Palczewski et al., 2000), shown in Figure 1.7 as purple spheres. The extracellular and transmembrane $\mathrm{Zn}^{2+}$ binding sites have been proposed to be the physiologically relevant ones (Park et al., 2007; Stojanovic et al., 2004), but X-ray absorption spectroscopy (Toledo et al., 2008), NMR spectroscopy (Patel et al., 2005) and proteolytic digestion (Shuster et al., 1992) have suggested the primary binding sites to be located in the EC domain. 
Figure 1.7 - Cartoon representation of dark state rhodopsin highlighting the presence of $\mathrm{Zn}^{2+}$ bound.

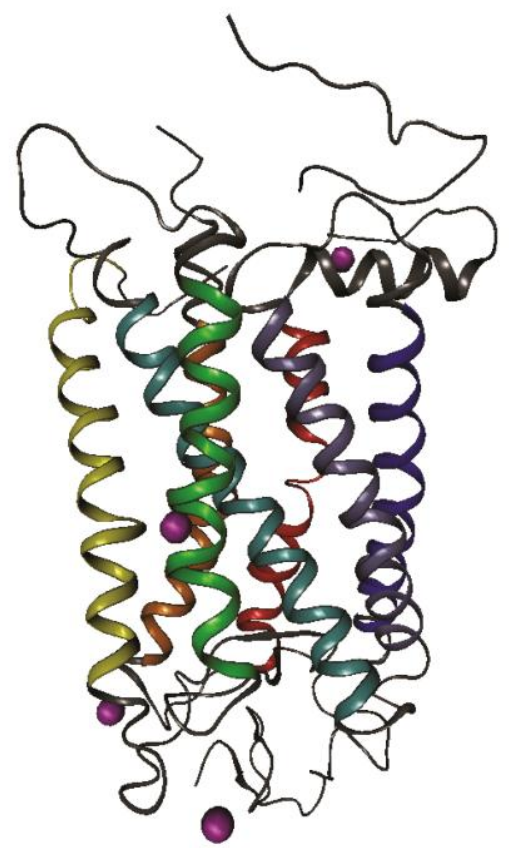

The four $\mathrm{Zn}^{2+}$ ions bound are represented as spheres and colored in magenta. Images were generated using VMD visualization software (Humphrey et al., 1996).

SOURCE: The structure shown is PDB identification 1L9H (Okada et al., 2002; Palczewski et al., 2000).

\section{$\underline{\text { Anthocyanins }}$}

Anthocyanins are plant pigments belonging to the large group of phenolic compounds, the flavonoids. They are abundant in a diet rich in fruits and vegetables. Anthocyanin consumption confers a broad spectrum of health benefits, especially in vision, where they have been implicated to have protecting, enhancing and restoring abilities. They also have anti-oxidant, anti-cancer, anti-diabetic, anti-inflammatory, anti-aging and cardioprotective properties (Bagchi et al., 2004; Kaushal and Khorana, 1994; Lila, 2004; Zafra-Stone et al., 2007). Recently, anthocyanins from blackcurrants were shown to enhance the regeneration of rhodopsin, directly linking anthocyanin action to vision-related functional effects (Matsumoto et al., 2003). In the dark, 11-cis retinal bound via Schiff base linkage to Lys296 in the TM domain of rhodopsin, gives rise to a chromophore with absorbance maximum at 500nm (Figure 1.8A). Illumination results in formation of the activated Meta II state of the protein with characteristic absorbance maximum at 380nm (Figure 1.8A), in which the alltrans retinal isomer is bound. Slowly - in the order of minutes - the Schiff base is hydrolyzed and all-trans retinal leaves the protein, forming opsin. This Meta II decay can be 
followed by tryptophan fluorescence (Figure 1.8B). The essay consists of illumination of the sample, and measuring the rate of fluorescence increase, as the tryptophans are exposed, corresponding to the rate of retinal release.

Opsin is then able to bind free 11 -cis retinal and the 500nm chromophore is restored. This process is referred to as regeneration. The faster rates of rhodopsin regeneration in the presence of anthocyanins are potentially of functional importance because under dim-light conditions it is critical to ensure that rhodopsin is quickly available after activation to participate in a renewed visual signal transduction cascade.

Figure 1.8 - Application of different spectroscopic methods to the analysis of rhodopsin.
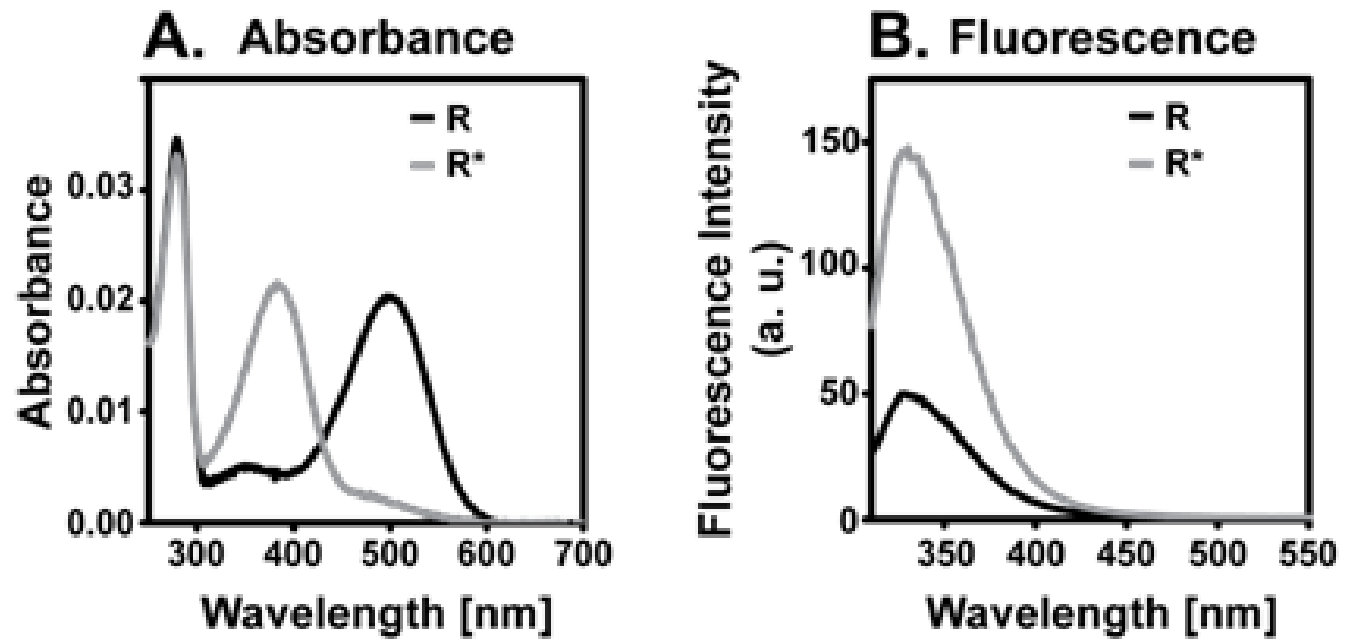

(A) Absorbance spectrum. For absorbance spectrum, rhodopsin in the dark (R; black line) and after illumination $\left(\mathrm{R}^{*}\right.$; grey line) are shown. The rhodopsin concentration is $0.5 \mu \mathrm{M}$. (B) Fluorescence emission spectrum (excitation wavelength $295 \mathrm{~nm}$ ) in the dark (R; black line) and 60min after illumination ( $\mathrm{R}^{*}$; grey line). The rhodopsin concentration is $0.5 \mu \mathrm{M}$.

SOURCE: Figure adapted from (Tirupula et al., 2009). With permission.

Anthocyanin cyanidin-3-O-glucoside $(\mathrm{C} 3 \mathrm{G})$ binds directly to rhodopsin in the dark and upon light activation, probably in its cytoplasmic domain (Yanamala, 2009). The apparent affinity of C3G for dark-adapted rhodopsin is $50-100 \mu \mathrm{M}$ based on ${ }^{1} \mathrm{H}$ NMR data recorded at $\mathrm{pH} 6$, but the affinities for light-activated and opsin states is likely higher and remain to be determined. Evidence was provided that the mode of interaction not only varies with activation-state but also with $\mathrm{pH}$, as $\mathrm{C} 3 \mathrm{G}$ exists in multiple chemical forms, namely the flavylium ion, the quinoidal base, the carbinol pseudobase and the chalcone, which are shown in Figure 1.9 (Veitch and Grayer, 2008). 
Figure 1.9 - Chemical structures of $\mathrm{pH}$ dependent equilibrium species of the cyanidin-3-glucoside.
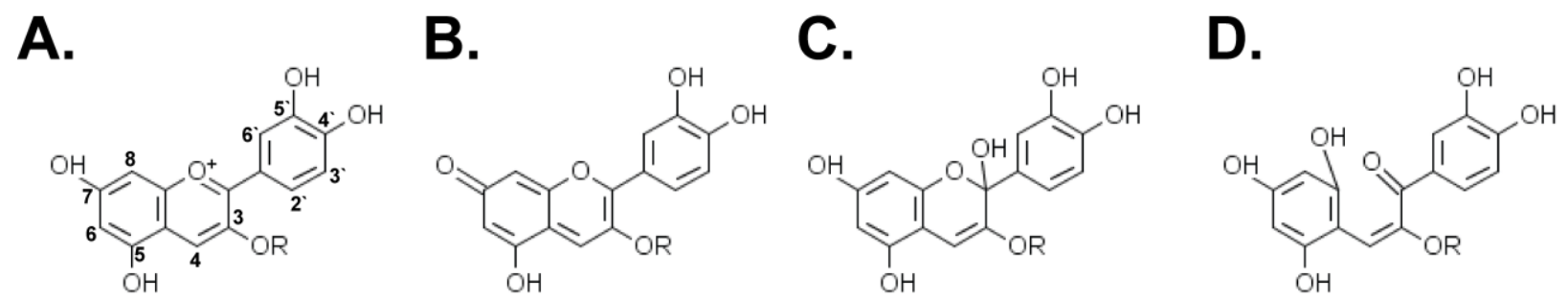

(A) Flavylium cation. (B) Quinoidal base example. (C) Carbinol pseudobase. (D) Chalcone. 'R' in the chemical structures of $\mathrm{C} 3 \mathrm{G}$ represents the glucoside sidechain.

SOURCE: Figure adapted from (Yanamala et al., 2009). With permission.

\section{$\underline{\text { Chlorin e6 }}$}

Porphyrins are an omnipotent class of naturally occurring compounds with many important biological representatives such as hemes and chlorophylls. The chlorophyll derivative chlorine e6 (Ce6) (Figure 1.10) is believed to enhance sensitivity of rhodopsin to red light in deep-sea fish rhodopsin (Douglas et al., 1999; Isayama et al., 2006).

\section{Figure 1.10 - Chemical structure of Ce6.}

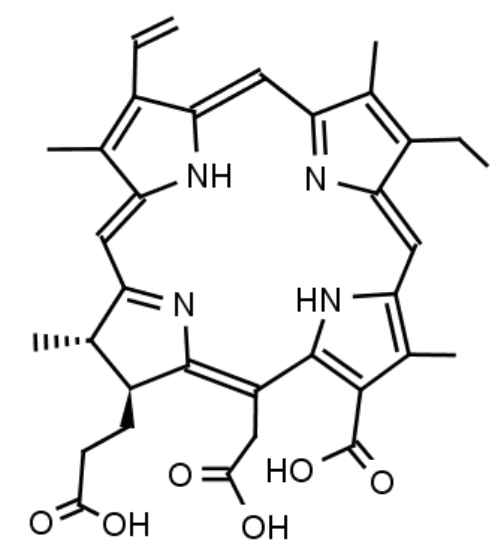

SOURCE: Balen, 2012

In vivo studies with salamander and mouse models have confirmed that Ce6 can effectively enhance vision in these animals (Washington et al., 2007). A decrease in the $500 \mathrm{~nm}$ peak of a UV-Vis spectrum of salamander rhodopsin was observed by illuminating the sample at $668 \mathrm{~nm}$, a wavelength at which $\mathrm{Ce} 6$ absorbs while rhodopsin has less response (Isayama et al., 2006). Additionally, mice administered with Ce6, showed a two-fold increase in electroretinogram b-wave amplitudes (the mass response to the eye) as a response to red and blue light (Washington et al., 2007). An energy transfer between Ce6 and 11-cis retinal 
has been proposed (Isayama et al., 2006). In vitro studies demonstrated that Ce6 physically binds to rhodopsin and modulates its structure and function (Yanamala, 2009).

\subsubsection{Retinitis pigmentosa}

Mutations in rhodopsin are associated with the retinal degeneration disease retinitis pigmentosa (RP). RP reflects a large group of genetically heterogeneous disorders that affect the photoreceptors and retinal pigment epithelium (RPE) diffusely across the entire fundus but begin with initial geographic involvement in either the periphery or the macula (Sieving, 2010). It is one of the major causes of blindness in the world, affecting 1 in 4000-5000 people (Kannabiran, 2008). The disease is marked by early rod photoreceptor dysfunction and

progressive degeneration of rods, which impairs vision in dim light and causes loss of peripheral vision, that is "tunnel vision" (Sieving, 2010), and later cones (Lam, 2005). This sequence of events explains why patients initially present night blindness, and only later in life presents diurnal visual impairment (Hamel, 2006). Typical RP is then described as rodcone dystrophy, where rods are more affected than cones. However, some of the allied forms primarily cause cone photoreceptor loss and initially manifest with a reduction in central visual acuity (Sieving, 2010).

The pathologic mechanism underlying the observation that at the latest stages in the disease retinal degeneration is observed is still not clear. Family-related retinal degeneration with intraneural retinal pigmentation was described as early as 1855 (Sieving, 2010), and although "retinitis" implies an inflammatory or infectious cause, histopathology studies shows no evidence of macrophage invasion or other inflammatory response in the photoreceptor layer or elsewhere in the retina (Sieving, 2010). Studies attributed a genetic basis to the majority cases of RP and recently concluded that apoptosis is the last stage contributing to photoreceptor cell death (Chang et al., 1993; Portera-Cailliau et al., 1994; Reme et al., 1998). Belmonte et al. (2006) showed that Rac1, a member of the GTPase family with very low molecular weight, may participate in the cell death process when the degeneration is mediated by light or inherited. Moreover, after degeneration, there was an increase in the activity of Rac1, and furthermore Rac1 expressing photoreceptors were shown to be TUNEL-positive. TUNEL is a common method for detecting DNA fragmentation, by labeling the terminal end of nucleic acids, which results from apoptotic signaling cascades. 
No evidence of racial or ethnic predisposition has been observed. However, men may be affected slightly more than women because of X-linked conditions (Sieving, 2010). The age-at-onset of symptoms and diagnosis is variable and can range from early childhood to mid-age adults. The diagnostic criteria is assessed through 4 parameters (Hamel, 2006); 1) Functional signs: which are characterized by night blindness as the first symptom, followed by photophobia; visual acuity is preserved in early and mid ages. 2) Visual field: marked by patchy losses of peripheral vision evolving to ring-shaped scotoma, tunnel vision is the last step. 3) Fundus: presence of pigmentary deposits similar to bone spicules in the peripheral retina; thinning of the retinal vessels; waxy pallor of the optic nerve; various degrees of retinal atrophy. 4) Electroretinogram: decrease of the amplitudes of a- and b- waves; scotopic system, rods, predominates over photopic system, cones.

Methods for clinical diagnosis are based on the presence of night blindness and peripheral visual defects, lesions in fundus, ERG changes, and progressive deterioration of all of these signs (Hamel, 2006). The ERG is the best method to detect the disease, since patients can be asymptomatic, for example presenting only nigh blindness and no other clinical signs.

\subsubsection{Types of retinitis pigmentosa}

RP is extremely heterogeneous, both clinically and genetically. Cases where there are no family histories evident are known as isolate, sporadic or simplex. By inheritance, RP can be classified in autosomal dominant (AD) (30\%), autosomal recessive (AR) (20\%) and Xlinked recessive (XL) (15\%) or sporadic/simplex traits $(30 \%)$, and be part of the group of Leber congenital amaurosis (5\%). These are among the more common modes of inheritance in RP and the relative prevalence of each form varies between populations (Kannabiran, 2008; Musarella and Macdonald, 2011). There are also rare forms of RP that are called X-linked dominant, mitochondrial and digenic (presents mutations in two different genes) (Musarella and Macdonald, 2011). Clinically, they are known as non-syndromic RP. Mutations in 16 different genes were found to cause autosomal dominant retinitis pigmentosa (ADRP) (Kannabiran, 2008). Autosomal dominant forms are usually the mildest forms, some cases starting after the age of 50, although severe disease can also appear (Hamel, 2006). The most frequent cause of ADRP is a mutation found in the rhodopsin gene, affecting 20-25\% of the patients, especially in North America and Europe. In North America, a single point mutation, Pro23His, accounts for $12 \%$ of the cases, although not significantly in Europe. Mutations on 
the RDS gene is the second most predominant set of mutations in RP, 3.5 to $9 \%$ were estimated followed by the RP1, which was estimated to be $4-8 \%$ of the cases. Other mutations appear to be less frequent and are implicated in less than 5\% of the cases (Kannabiran, 2008).

Autosomal recessive retinitis pigmentosa (ARRP) is a group of disorders that includes juvenile and early-onset forms of RP. This group overlaps with another neurodegenerative disease called Leber's congenital amaurosis (LCA). 21 genes were identified to cause ARRP. However, the frequency of mutations from all of these genes accounts for approximately a third of ARRP patients, with individual genes contributing to less than $5 \%$ of disease (Kannabiran, 2008). There are two genes that were found to cause X-linked RP, RP2 and RP3 (known as RP GTPase regulator (RPGR)). RP2 account for 10-20\% of recessive XLRP and mutations in RPGR account for $50-80 \%$ of recessive XLRP. Another six loci were also mapped but the genes were not identified yet (Kannabiran, 2008). X-linked forms also start early and are frequently associated with myopia. Transmission of the disease is recessive in most cases; however there are some families in which dominant inheritance with affected females is found (Hamel, 2006).

Many syndromes are associated with different types of pigmentary retinopathies, and are known as syndromic RP. The most frequent are the Usher and Bardet Biedl syndromes (BBS). Usher syndrome is the most frequent and is typically associated with neurosensory deafness. BBS is marked by obesity (already in childhood), mental retardation or mild psychomotor delay, post axial polydactyly, hypogenitalism and renal problems and RP associated, frequently as cone-rod type of dystrophy (Hamel, 2006). Other less frequent syndromes encompass those that cause renal abnormalities such as Senior Loken syndrome (SLS) and Alport syndrome, dysmorphic syndromes such as Cohen, Jeune and Cockayne syndromes, metabolic syndromes such as Methylmalonic aciduria with homocystinuria, Abetalipoproteinemia (Bassen Korntzweig disease), Bietti's disease, Cystinosis, Mucopolysaccharidoses, Zellweger (cerebro-hepato-renal) syndrome, Hyperoxaluria type I with retinal atrophy in spots, Neonatal adrenoleukodystrophy with leopard spots fundus, Infantile Refsum disease, Adult Refsum disease, Peroxisomal disorders other than Refsum disease, Peroxisomal disorders other than Refsum disease and neurological disease, neuronal ceroid lipofuscinosis, Joubert syndrome, autosomal dominant cerebellar ataxia type II (SCA7), Myotonic dystrophy and Hallervorden-Spatz syndrome (Hamel, 2006). 


\subsubsection{Current therapies}

Although innumerous studies have been performed, there is no effective therapy to treat RP. Meanwhile, many studies are focusing on alternative therapies to prevent, treat or slow down the disease.

In order to slow down the degeneration process, light protection and treatment with vitamins is recommended. Animal studies suggested that light exposure was damaging for the transgenic photoreceptors (Wang et al., 1997), and it is recommended to patients with pigmentary retinophaties to avoid direct light exposure, by either wearing dark glasses or yellow-orange spectacles, which minimizes photophobia. The use of eye shades and lateral protection is also recommended to protect against light coming from the sides (Hamel, 2006). Treatment with vitamins $\mathrm{A}$ and $\mathrm{E}$ are believed to protect the photoreceptors by trophic and antioxidant effects. A clinical trial of nutritional supplements for adults diagnosed with the common forms of RP was conducted (Kupfer, 1993). The course of retinal degeneration was monitored by electroretinogram recordings. On average, patients taking 15,000 IU of vitamin A daily showed slower, and those taking 400 IU of vitamin E showed faster retinal degeneration. The molecular mechanism of the Vitamin A effect is the rescue of correctly folded rhodopsin by retinal. However, 15,000 IU only slowed and could not prevent retinal degeneration, and a daily vitamin A intake exceeding 25,000 IU over the long-term is toxic. Later, similar studies were conducted and showed complementary or adverse results (Berson et al., 1993a, 1993b, 2004, 2010).

Different therapies have therefore been explored (Kannabiran, 2008). Gene therapy methods have been geared towards correction of the specific gene mutated, by means of gene replacement for recessive gene mutations or suppression and replacement of mutant mRNAs for dominant gene mutations. Encapsulated cells releasing neurotrophic factors have been implemented with the aim of promoting survival and growth of photoreceptors. Antiapoptotic factors have been introduced by gene-delivery systems. Finally, stem cell transplantation has been considered. Each approach is briefly out-lined below.

Gene therapy is a promising therapy for many inherited human diseases. The technique requires efficient genotyping methods for identification of the implicated gene (Hamel, 2006). Ocular gene therapy (OGT) has been successfully tried in different animals and humans. First, identification of the genetic cause of the RP and genotyping of the patients was performed in order to proceed with the genetic modification of mutant ocular cells to 
produce therapeutic effects. The strategy of the therapy differs accordingly with the type of disease or mutation target, and was already applied successfully in mouse (Bennicelli et al., 2008; Tan et al., 2009), rats (Drenser et al., 1998; Lewin et al., 1998; Weber et al., 2003), dogs (Acland et al., 2001; Veske et al., 1999; Weber et al., 2003), non-human primates (Weber et al., 2003), and humans (Bainbridge et al., 2008; Hauswirth et al., 2008; Maguire et al., 2008, 2009). Due to different outcomes from patients, more tests still need to be done in order to prove the efficacy for all patients.

Retinal implants using either epiretinal or subretinal implants is also a good therapy and have shown benefits in many tests with RP patients (Caspi et al., 2009; Humayun and de Juan, 1998; Yanai et al., 2007) and animals models (Chow and Chow, 1997; Zrenner et al., 1997). Clinical trials in human patients are already in Phase II, but still being improved.

Neurotrophic factors showed protective effects on photoreceptors degeneration, including neurotrophic factor (CNTF), Glial cell-line derived neurotrophic factor (GDNF), cardiotrophin-1, brain-derived neurotrophic factor (BDNF) and basic fibroblast growth factor (bFGF) (Hamel, 2006; Musarella and Macdonald, 2011) in animals (Bok et al., 2002; Frasson et al., 1999; Li et al., 2010; Liang et al., 2001; Tao et al., 2002) and are in the trial phase in humans (Sieving et al., 2006). Phase I and II are currently underway for treatment of atrophic macular degeneration and RP.

Retinal transplantation consists of the transplantantion of sheets of retinal cells, layers of photoreceptors or a complete retina (Hamel, 2006; Musarella and Macdonald, 2011). Alternatively, transplantation of photoreceptor precursors has also been tried (Musarella and Macdonald, 2011). Transplants in animal models and humans showed that transplanted material does survive but not all presented evidence of a therapeutic effect (Gal et al., 2000; MacLaren et al., 2006; Radtke et al., 2008).

Stem cell treatment is being used to regenerate degenerated retinas, based on its capability to give rise to specialized cells and also its capability of self-renewal (Lund et al., 2006). The donor cells and the host recipient are two major aspects of the procedure (Rivas and Vecino, 2009). Hippocampal, embryonic and bone marrow cells have been tested in models of retinal degenerative disorders and have showed high migratory and differentiation capacity. In contrast, the use of retinal stem/progenitor cells in suspension or as neurospheres exhibit poor migration, but were successful in expressing retina specific markers after transplantation. Although progress is being made, extrapolation to humans is yet to be demonstrated. 
Neuroprotection using antiapoptotic factors or inhibitors of apoptosis is also being explored (Hamel, 2006; Kannabiran, 2008). Studies in mouse and rat models have shown protection of photoreceptor cells (Bennett et al., 1998; Leonard et al., 2007; Liu et al., 1999; Petrin et al., 2003).

\subsubsection{Rhodopsin as a target in retinitis pigmentosa}

The causes for RP are heterogeneous, and mutations in many different genes can lead to RP. The first of these genes that has been identified as a genetic cause for RP was the rod photoreceptor rhodopsin. Now more than 150 mutations in rhodopsin have been identified in different RP patients (Cooper et al., 2007). From these, 92 are single point mutations in 64 amino acid positions (Figure 1.11). This is a remarkable number considering that rhodopsin is a protein with a total length of only 348 amino acids. 42 of these RP mutations have been studied in vitro and 31 of them (>70\%) were found to cause misfolding of rhodopsin.

Many studies have focused on understanding the molecular mechanism underlying rhodopsin mutations leading to RP development. The majority of the mutations studied previously focused on the EC and TM domains of rhodopsin (Dryja et al., 1993, 1995). Most of these mutations mediate formation of an incorrect disulfide bond, leading to an incorrectly folded protein that cannot bind 11-cis retinal (Kaushal and Khorana, 1994; Ridge et al., 1995). This finding suggested a structural coupling between the EC and TM regions, which is important for the correct folding of rhodopsin.

Since the discovery of misfolding of rhodopsin as one of the leading causes for RP (Kaushal and Khorana, 1994), much effort has concentrated on rescuing correctly folded rhodopsin by adding excess Vitamin A or retinoid derivatives. Recently, a clinical trial of

nutritional supplements for adults with common forms of RP was conducted, and demonstrated that vitamin A treatment alone cannot cure RP (see above). 
Figure 1.11 - Secondary structure model of rhodopsin showing the positions of the mutations that cause RP and congenital stationary night blindness.

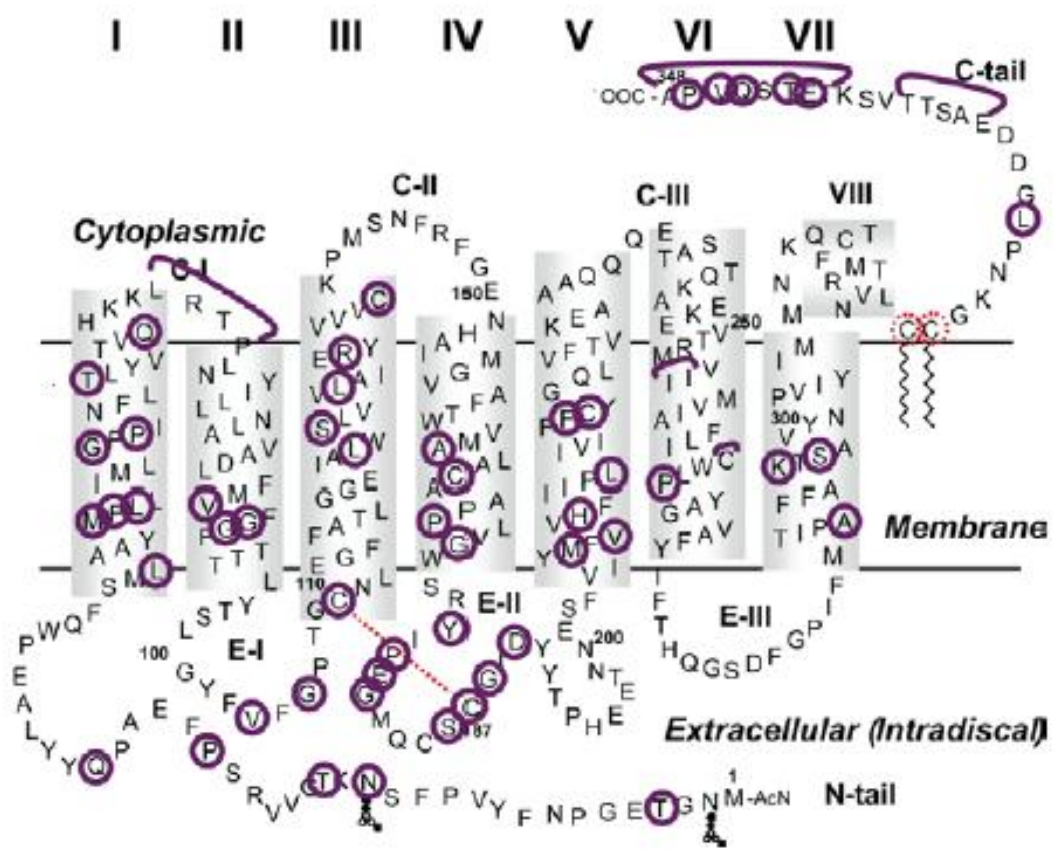

SOURCE: Balen, 2012

\subsubsection{Animal models of retinitis pigmentosa}

Hereditary retinal degeneration affects human and other animals. The range of diseases that affect animals can be due to natural occurrence or obtained through genetic manipulation. These various mutations are of great importance as model system for the study of the disease evolution, pathogenesis and strategies for treatment. Specifically for RP, there are many animal models available and many of them mimic the disease that is found in humans.

The list of animal models that present mutations that occur naturally includes dogs, cats and chicken. Natural and induced mutations are studied in mouse, rats and pigs. These animal models were reviewed recently (Rivas and Vecino, 2009) and are summarized here.

Canine animal models of RP are considered the best models due to the similarity between dog and human eye disorders. Furthermore, since eye anatomy and size in dogs is similar to human, the same apparatus for physical studies can be used for dogs as that for humans. All canine diseases present rod-cone degenerations, where rod disease, dysfunction, and death precede the loss of cones. These diseases are analogous to human RP and are called Progressive Retinal Atrophy (PRA). The genes mutated include PDE6A and PDE6B encoding 
the $\alpha$ and $\beta$ subunits of the cGMP specific phosphodiesterase (autosomal recessive RP), RPE65 gene (leber congenital amaurosis), the RP GTPase regulator RPGR (X-linked RP) and rhodopsin RHO (autosomal dominat RP). A list of available canine models is provided below:

- erd - canine early retinal degeneration

- rcd-1 - rod-cone dysplasia type 1 - and rcd-2 - rod-cone dysplasia type 2

- RHO - rhodopsin, visual pigment of rod photoreceptor

- RPE65 - membrane-associated protein

- RPGR - mutations in the RP GTPase regulator

- $\quad$ RPGRIP1 - mutations in the RP GTPase regulator-interacting protein 1

Cats are good models too since they have a manageable eye size for examination and manipulation. Their visual neurophysiology is well characterized and their eyes are physiologically and anatomically similar to human. There are only two forms of feline PRA that are well studied:

- $\quad$ rdAc - autosomal recessive rod-cone degenerative disorder

- Rdy model - autosomal dominant early-onset retinal degenerative disease

Chicken eyes are also useful to study since they can be compared with human eyes in size, facilitating pathological examination. However, chicken eyes are very different from human eyes from a number of perspectives. In particular, the chicken eye is cone-dominated rather than rod-dominated. However, the conservation of gene order between chicken and humans is similar to that between human and mice genome, making this a potential model for the study of spontaneously occurring inherited blindness disease. There are two models of chicken that presents retinal degeneration:

- $\quad$ rd - retinal degeneration chicken strain

- $\quad$ rdd - retinal dysplasia and degeneration

Mice are the most studied animal models, and the rod-less mouse was the first animal model where retinal degeneration was described. Mouse and human have a genomic similarity of about $90 \%$, and gene correlation can be precisely achieved. Advantages in the use of mouse 
include the short life span (about 2 years), availability of protocols that are well established and the ease at which new single gene diseases can be mapped. Available mouse models are:

- Peripherin rds - retinal degeneration slow, rds-peripherin null mutation

- $\mathrm{Rd}-$ retinal degeneration, which includes $\mathrm{rd} 1, \mathrm{rd} 4, \mathrm{rd} 8, \mathrm{rd} 10, \mathrm{rd} 12$

- CEP290: rd16 - novel centrosomal protein

- I-255/256 - mutant opsin gene with 3-bp deletion of isoleucine at codon 255/256

- Knockout RPE65 (RPE65-/-) - accumulates retinyl esters in the RPE and lacks 11-cis retinal

- $\mathrm{P} 347 \mathrm{~S}$ - proline-347 to serine mutation in rhodopsin

- Peripherin-rds 307 - mice heterozygous for the codon 307 mutation

- Q334ter - transgenic mice that express a truncated rhodopsin due to a mutation resulting in a stop codon at position 344

- Knockout rho (rho-/-) - mutations in the rhodopsin gene (have no functional rhodopsin gene)

- VPP - mice express a mouse opsin gene containing three point mutations within a seven amino acid sequence near the $\mathrm{N}$-terminus of the molecule

$\circ \mathrm{P} 23 \mathrm{H}$ - histidine for proline mutation

○ V20G - glycine for valine mutation

○ $\mathrm{P} 27 \mathrm{~L}$ - leucine for proline mutation

Rats exhibit retinal degeneration very similar to the retinal degeneration observed in humans. The benefits of using rat photoreceptors are that they have been well studied, therefore extensive knowledge of basic biological mechanisms is known. Morphologically, the rat eye is several times larger than the mouse, which simplifies surgical manipulations and electroretinographic evaluation; they also breed as rapidly as mice, generating large litters in a short gestational time. The following lines are available:

- $\mathrm{RCS}$ - Royal College of Surgeons. Retinal pigment epithelium (RPE) is unable to phagocytose shed photoreceptor outer segments.

- $\mathrm{P} 23 \mathrm{H}$ - histidine at proline 23 position in rhodopsin. Rat carries a mutant mouse opsin gene in addition to the endogenous native opsin genes 
- S334ter - the opsin transgene contains a termination codon at residue 334, resulting in the expression of a rhodopsin protein lacking the last $15 \mathrm{C}$-terminal amino acids

Pigs are genetically engineered animal models. Pig eyes are similar in size to the human eyes. They have a similar number and distribution of rod and cone cells as humans, which makes them an excellent model for RP. Among other large mammals, the porcine retina is even more similar to the human retina in size, and tools applied for diagnostics can be used without adaptations.

- P347L - lysine for proline at position 347 in rhodopsin

- $\quad \mathrm{P} 347 \mathrm{~S}$ - serine for proline at position 347 in rhodopsin

The figure below represents the time of initiation of retinal degeneration in each animal model (Figure 1.12). Knowledge of the different degeneration times is important in order to decide which model is more relevant to specific studies such as conducted as part of this thesis. 
Figure 1.12 - Schematic representation of the initiation of the retinal degeneration in each animal model.

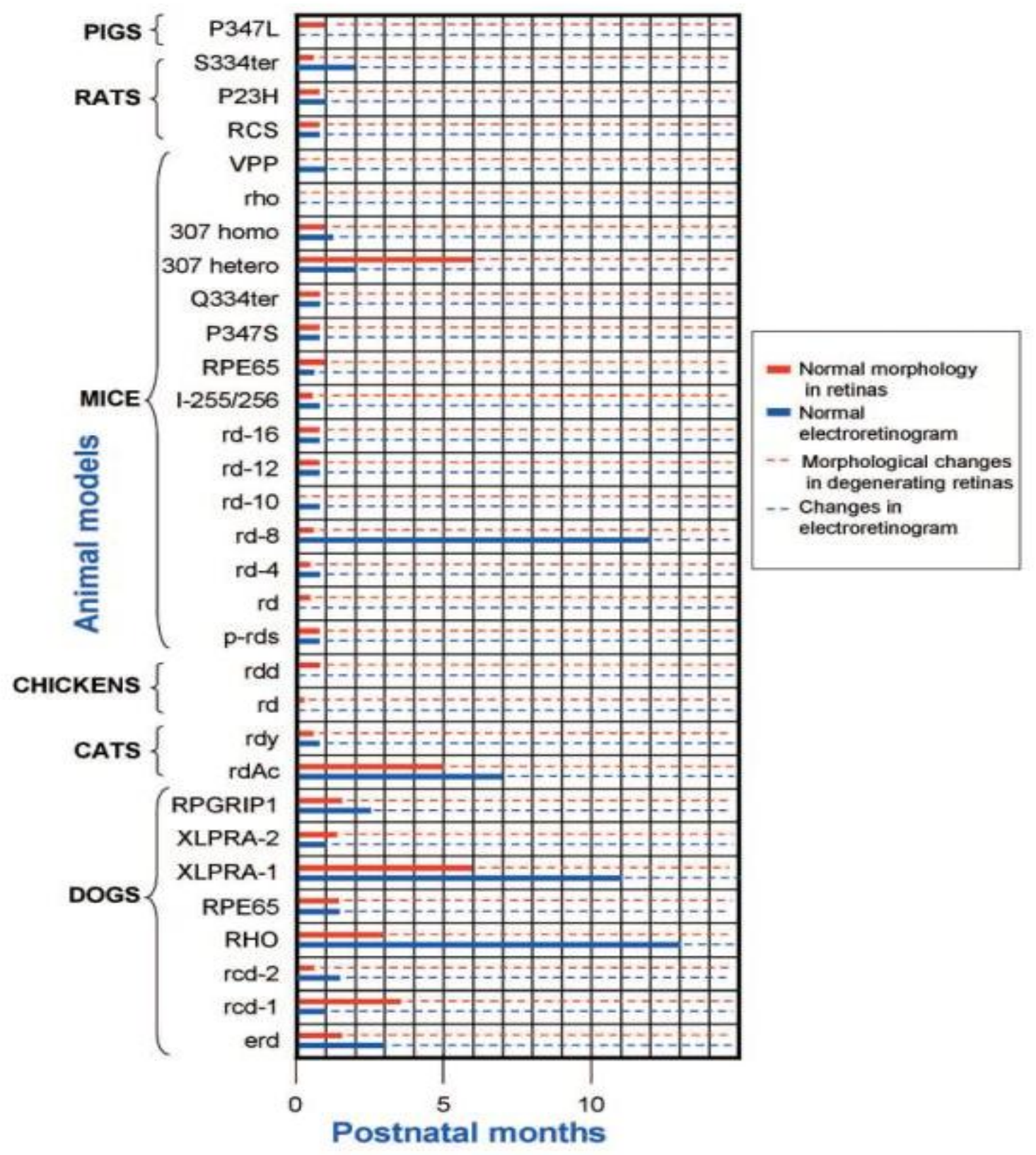

Continuous lines: normal retina (non-degenerating); discontinuous lines: degeneration of the retina; Red: morphological states; Blue: electroretinographic state; each square represents a postnatal age of the animal (months).

SOURCE: Figure is taken from (Rivas and Vecino, 2009). With permission. 


\subsection{OPEN QUESTIONS AND THESIS OBJECTIVES}

\subsubsection{Open questions}

Previous studies by Khorana and coworkers have addressed the identification of the amino acids stabilizing rhodopsin and have established that there is tight coupling between the extracellular, transmembrane and cytoplasmic domains in rhodopsin (Khorana, 2000). Disruption of this coupling causes irreversible misfolding. Recent simulations of misfolding of the rhodopsin tertiary structure have provided a mechanistic understanding of these findings (Rader et al., 2004). A major core of residues contributing to rhodopsin stability was identified at the interface between the extracellular and transmembrane domains. A small additional region at the conserved DERY motif in the cytoplasmic domain was also implicated in playing a role in rhodopsin stability. More than $90 \%$ of the residues in the large core cause misfolding upon mutations (Rader et al., 2004). Furthermore, recent findings with RP patients and molecular studies have correlated these molecular studies with RP patients studies (Iannaccone et al., 2006). This indicates that it may be possible to develop new avenues for RP treatments based on stabilization of rhodopsin structure. Such new avenues are needed because there is, so far, still no cure or treatment for RP.

Here we explore novel approaches for the stabilization of opsin/rhodopsin through small molecules. Rhodopsin has been shown to be a receptor for anthocyanins (Lila, 2004) and its spectral range can be extended by interacting with porphyrin compounds (Washington et al., 2004). Members of the flavonoid group of phytochemicals, anthocyanins are mostly found in teas, honey, wines, fruits, vegetables, nuts, olive oils, cocoa and cereal. Anthocyanins pigments and the flavonoids associated are known to protect against many kinds of diseases. The enhancement of night-time vision by anthocyanins is known (Lila, 2004). Porphyrins are an omnipotent class of naturally occurring compounds with many important biological representatives such as hemes and chlorophylls. Previous evidence from our laboratory suggests that porphyrin and anthocyanin compounds both stabilize rhodopsin and/or opsin (Yanamala, 2009).

In addition, studies have shown that providing patients with more retinal, via administration of Vitamin A, can only partially slow down RP. So far, many alternative approaches have been proposed, but none have an entirely positive effect on reversing the 
disease. The hypothesis that molecules other than retinal can alone or in conjunction with retinal rescue folded rhodopsin may lead to new treatments of RP.

\subsubsection{Objectives and Approaches}

This thesis aims to investigate the hypothesis that molecules other than retinal can rescue folded rhodopsin and/or reduce photoreceptor cell death, which could potentially lead to new treatments for RP. To address the open questions, the following specific aims are proposed:

\section{Specific aim 1: In vitro molecular characterization of WT and different rhodopsin mutants}

A study comparing two RP mutations N15S and P23H with WT rhodopsin was performed. Rhodopsin and mutants were expressed in COS-1 and HEK-293 cells followed by purification after reconstitution with 9-cis or 11-cis retinal, during or after expression in the cells. This provided a quantitative understanding of the role of retinal in the rescue of folded rhodopsin. Using thermal denaturation, Total Reflectance Fourier Transform Infrared (ATR/FT-IR) and fluorescence spectroscopy, SDS-PAGE mobility, glycosylation studies, and subcellular localization using confocal microscopy, a quantitative comparison of molecular properties of the mutants as compared to the WT was assessed.

\section{Specific aim 2: Effects of ligand binding on rhodopsin structure and stability}

First, stability studies using the protein rhodopsin from native source was carried out. Next, the effect of different compounds such as metal ions, porphyrins and anthocyanins were tested in order to assess their effect on the stability of rhodopsin. UV-visible, fluorescence and CD spectroscopy were used in order to compare of molecular properties of the WT rhodopsin alone and in presence of the different ligands tested.

\section{Specific aim 3: In vivo drug treatment of light-damaged and mutant rats with hereditary RP disease}

The aim was to test whether Ce6 is able to prevent apoptosis in Sprague Dawley rats submitted to light-induced degeneration, and in $\mathrm{P} 23 \mathrm{H}$ and S334ter rat models of RP. Morphological and functional (ERG) analyses were performed. 


\section{CHAPTER 2: EXPERIMENTAL PROCEDURES}

\subsection{EXPRESSION AND PURIFICATION OF RHODOPSIN}

\subsubsection{Preparation of rhodopsin from bovine retinae}

All procedures involving rhodopsin were carried out under dim-red light conditions. Frozen bovine retinae were purchased from J. A. Lawson Co. (Lincoln, NE). Each retina yielded 1.0 to $1.5 \mathrm{mg}$ of rhodopsin. Depending upon the amount of rhodopsin required for an experiment the corresponding number of retinae was taken. The retinae were thawed and then suspended in equal volume of PBS buffer $\left(137 \mathrm{mM} \mathrm{NaCl}, 2.7 \mathrm{mM} \mathrm{KCl}, 1.8 \mathrm{mM} \mathrm{KH} \mathrm{PO}_{4}, 10 \mathrm{mM} \mathrm{Na}_{2} \mathrm{HPO}_{4}\right.$ ( $\mathrm{pH}$ 7.2)). Retinae were mixed using a spatula and then transferred in small quantities to a Dounce homogenizer of appropriate dimensions depending on the volume in use. The retinae were then homogenized until no clumps were visible. After homogenization, dodecyl maltoside (DM) was added from a $10 \%$ stock solution in water to obtain a final concentration of $1 \%$. This mixture was then end-over-end mixed at $4{ }^{\circ} \mathrm{C}$ for $1.5-2.0$ hours. This was followed by centrifugation of DM solubilized retinae at 35,000 rpm in a 70Ti rotor using a Beckman ultracentrifuge to remove unsolubilized components. The pellet was discarded and an aliquot of the supernatant was used to quantify the amount of rhodopsin present by absorbance spectroscopy (described in Section 2.3.1.). Depending on the amount of rhodopsin estimated and the binding capacity of antibody (1D4) derivatized sepharose beads (described in Section 2.1.8.), which were used for purification of rhodopsin, were added to the supernatant. Solubilized retinae with 1D4 beads were then end-over-end mixed at $4{ }^{\circ} \mathrm{C}$ for 4 - 16 hours.

Following this, purification of rhodopsin was carried out with a nonapeptide as described in Section 2.1.8(c). 


\subsubsection{Expression in COS-1 cells}

\subsubsection{Construction of rhodopsin mutant genes in pMT4 for transient transfection}

Single amino acid replacement mutants $\mathrm{P} 23 \mathrm{H}$ and N15S were prepared by a two-step PCR mutagenesis technique using the synthetic bovine opsin gene in the expression vector pMT4 (Oprian et al., 1987). The first step involved a PCR reaction to create two fragments for each mutant. One fragment was created using the upstream universal primer containing an EcoRI restriction site (underlined) 5'-TATGAATTCCACCATGAACGGGACCGAGGGGC-3' and a second primer specific for each of the single codon replacement. The second fragment was generated from the primer specific for each codon replacement and the downstream universal primer that contains a NotI site (underlined):

\section{5'-TATTGCGGCCGCTTAGGCAGGCGCCAGTTG3'.}

The primers used were the following (the desired mutation site is underlined):

For P23H: Upstream: 5'-GTGGTGCGCAGCCACTTCGAGGCT-3'

Downstream: 5'-CGGAGCCTCGAAGTGGCTGCGCAC-3'.

For N15S: Upstream: 5'-GTTCCTTTCTCCTCCAAGACGGGC-3'.

Downstream: 5'-CACGCCCGTCTTGGAGGAGAAAGG-3'.

The second step involved a PCR reaction to generate the fragments using the universal primers and templates created from the first step. The PCR products from the second step were used to replace the WT opsin gene in the pMT4 vector using EcoRI and NotI restriction sites. The final pMT4 vector carrying mutant opsin genes were confirmed by DNA sequencing.

\subsubsection{COS-1 cell culture and transient transfection}

COS- 1 cells were transiently transfected with the WT and mutant opsin carrying plasmids as described in (Oprian et al., 1987) with the minor modifications described below.

Cells stored in liquid nitrogen were thawed as quickly as possible by placing vials in a $37{ }^{\circ} \mathrm{C}$ water bath after removal from liquid nitrogen. The cells were transferred to a $15 \mathrm{ml}$ Falcon tube and slowly (over 2 minutes) $10 \mathrm{ml}$ of DMEM buffer (DMEM supplemented with 100 units/ml Penicillin/Streptomycin (P/S), 10\% bovine serum (BS) was added to allow slow 
diffusion of the cryo-preservative DMSO from the cell. Cells were pelleted at $1000 \mathrm{rpm}$ for 10 minutes in a Sorvall Legend R T benchtop centrifuge, the supernatant was aspirated and $10 \mathrm{ml}$ of fresh DMEM buffer was added. The cells were resuspended and transferred to a $10 \mathrm{~cm}$ tissue culture dish and incubated at $37{ }^{\circ} \mathrm{C}, 5 \% \mathrm{CO}_{2}$ (Nuaire DH Autoflow) in DMEM buffer. DMEM medium was purchased as a powder mixture (GIBCO) the compounds are described in the end of this chapter. After dissolving, it was supplemented with $3.7 \mathrm{~g} \mathrm{NaHCO}_{3}$ per liter of media. Cells were maintained at about $90 \%$ confluence maximum to avoid damage to the cells by overgrowth. Cells were fed every three to four days, until $\sim 80-90 \%$ confluence was reached.

Cells were split 1:5 to 1:20. Plates $(10 \mathrm{~cm}$ and $15 \mathrm{~cm}$ diameter $)$ were washed with $10 \mathrm{ml}$ of PBS buffer, $1 \mathrm{ml}$ of $0.05 \%$ Trypsin-EDTA was added to a $10 \mathrm{~cm}$ dish and $2 \mathrm{ml}$ to a $15 \mathrm{~cm}$ dish. After incubation at $37^{\circ} \mathrm{C}$ for 1 minute, the plates were agitated until the cells detached from the surface (10 and $15 \mathrm{~cm}$ dishes). $9 \mathrm{ml}$ (10 cm dish) and $8 \mathrm{ml}(15 \mathrm{~cm} \mathrm{dish})$ of DMEM buffer (DMEM supplemented with 100 units/ml Penicillin/Streptomycin (P/S), 10\% BS) were added to inactivate trypsin. Cells were removed from the plates by pipetting up and down 3-10 times and transferred into new dishes of appropriate size followed by thorough mixing on the new plate by tilting.

For long-term storage of COS-1 cells, 70-90\% confluent plates $(15 \mathrm{~cm})$ were trypsinized as described above. After centrifugation at $1000 \mathrm{rpm}$ for 10 minutes, the supernatant was discarded and the pellet was resuspended in $5 \mathrm{ml}$ of 10\% DMSO in buffer (DMEM supplemented with 100 units/ml Penicillin/Streptomycin (P/S), 10\% BS)) medium. $1 \mathrm{ml}$ aliquots were prepared in cryogenic storage vials (Nunc) and placed in cryofreezing container (Nalgene). The boxes were kept at $-70{ }^{\circ} \mathrm{C}$ in freezers overnight where the cooling rate was $1{ }^{\circ} \mathrm{C} /$ minute. The following day, the vials were transferred to liquid nitrogen.

Cells on confluent $15 \mathrm{~cm}$ dishes were transiently transfected with the pMT4 vector as follows. All solutions were equilibrated at $37{ }^{\circ} \mathrm{C} .15 \mu \mathrm{g}$ plasmid-DNA (pMT4) per plate was added to $10 \mathrm{ml}$ of a solution of $0.25 \mathrm{mg} / \mathrm{ml}$ DEAE-dextran, $0.1 \mathrm{M}$ Tris-HCl $\mathrm{pH} 8$ in DMEM medium without BS. The cells were aspirated, washed with $25 \mathrm{ml}$ of previous DMEM medium without bovine serum and incubated with the DNA-mixture for 6 hours at $37^{\circ} \mathrm{C}$. After 6 hours of incubation, the DNA mix was removed and without washing $2 \mathrm{ml}$ of shock buffer containing 10\% DMSO and $6 \mathrm{mM}$ Dextrose in PBS buffer was added and incubated for 2 minutes. Shock buffer was removed after 2 minutes and $15 \mathrm{ml} 0.1 \mathrm{mM}$ chloroquin in DMEM buffer without BS 
medium was added to the cells. The cells were then incubated for 2 hours. After aspirating the chloroquin solution, and washing with $25 \mathrm{ml}$ DMEM buffer without BS medium, the cells were incubated overnight with $30 \mathrm{ml}$ DMEM buffer medium (DMEM supplemented with 100 units/ml Penicillin/Streptomycin (P/S), 10\% (BS) ). Total incubation time starting with the addition of DNA was 55-72 hours. After aspirating the medium, cells were washed with $10 \mathrm{ml}$ PBS buffer and then scratched off the plates in $2 \mathrm{ml}$ PBS buffer containing benzamidine $(0.005 \%)$ and PMSF $(0.7 \mathrm{mM})$ per plate. Subsequent manipulations were performed on ice.

\subsubsection{Expression of rhodopsin in HEK293 cells}

\subsubsection{Construction of rhodopsin expression plasmid using pACMV-tetO}

$\mathrm{N} 15 \mathrm{~S}$ and P23H mutant opsin genes were created in the pMT4 vector as described in Section 2.1.2.1. These opsin genes were moved from the pMT4 vector to the pACMV-tetO vector for stable transfection in HEK 293 cells. The pACMV-tetO vector, was originally developed by Dr. Philip Reeves (Reeves et al., 2002a, 2002b). (Figure 2.1). A tetracycline (tet)regulated gene expression system, in mammalian cells was developed by fusing the bacterial tet repressor (TetR) with the activating domain of viral protein 16 (VP-16) (Gossen and Bujard, 1992), a tetracycline controlled transactivator was generated that enhanced transcripton of a gene from CMV-tetO promoter sequences. In another system developed by Yao et al. (Yao et al., 1998), a full-length CMV promoter is fused to two tetO sequences in tandem, which is tetracycline inducible and is based on TetR repression. Reeves et al. (Reeves et al., 2002) modified this system to generate a new vector pACMV-tetO, to express mutant opsin genes. 
Figure 2.1 - pACMV-tetO.

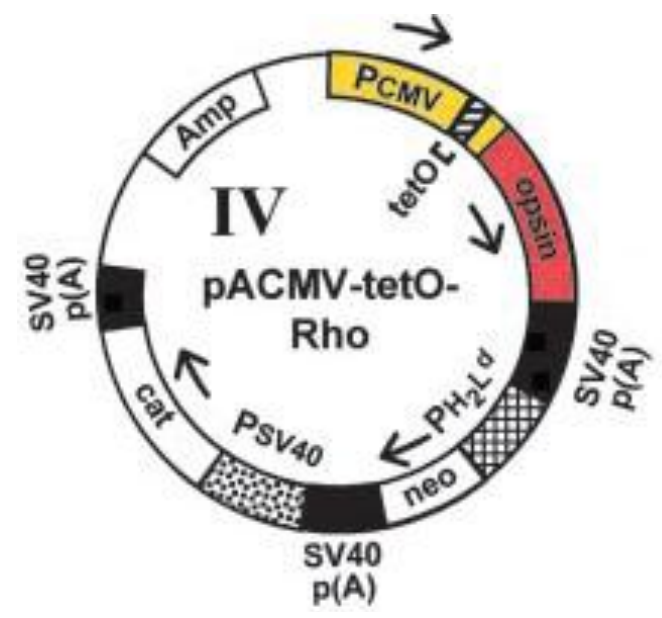

The figure shows the promoter (CMV-tetO) and other important regions in the vector.

SOURCE: Figure is taken from (Reeves et al., 2002). With permission.

In order to excise the opsin gene from the pMT4 vector, two unique restriction sites were required in pMT4 and pACMV vectors. Both the vectors had NotI and KpnI sites in common. However, the unique KpnI site in pMT4 is a few bp away from the starting codon within the opsin gene fragment and thus had to be moved to the start of the opsin gene by site-directed mutagenesis. The QuickChange site directed mutagenesis kit from Stratagene (Cat No 200518-5) was used to do so. This enabled excising the opsin gene by digesting with KpnI and NotI and then ligating into pACMV-tetO vector which was also cut by the same two enzymes. For the restriction digestion reactions, sequential digestion was set up with KpnI followed by NotI as per the reaction mix provided by NEB for each of these enzymes. For the ligation reaction, different vector to insert ratios of 1:6, 1:9 and 1:12 were tested for each mutant. Quick ligase enzyme (NEB) was added to the ligation mix and was kept at RT for 1 hour and then transformed into DH5 $\alpha$ cells and grown on agar plates overnight in a $37^{\circ} \mathrm{C}$ incubator.

Colonies from the above step were picked and used to grow a $1.5 \mathrm{ml} \mathrm{LB}$ media culture containing $100 \mu \mathrm{g} / \mathrm{ml}$ ampicillin. After growth at $37^{\circ} \mathrm{C}$ for 14 hours, DNA was purified from the cultures using Qiaprep spin miniprep kit (Qiagen, MD). The steps for purification were followed exactly as given in the instruction manual and purified DNA was dissolved in sterile water. The purified DNA was subjected to restriction digestion with KpnI and NotI. The clones showing fragments of appropriate size corresponding to opsin and the vector backbone were selected and their sequences were confirmed by the core sequencing facility at the University of Pittsburgh. 


\subsubsection{Expression of HEK293 stable cell lines containing the opsin mutant genes}

pACMV-tetO vectors containing the mutant opsin genes were used to stably transfect HEK293 cells. Routine growth, splitting and trypsinization, long-term storage and reviving from frozen stocks were as described for COS-1 cells in Section 2.1.2(b). HEK293S cells were transfected by the method of Chen and Okayama (Chen and Okayama, 1987) as modified by O’Mahoney and Adams (O'Mahoney and Adams, 1994). Stable cell lines expressing the opsin genes at high levels were selected using elevated concentrations of geneticin $(2-3 \mathrm{mg} / \mathrm{ml})$ as described (Reeves et al., 1996).

Frozen HEK 293 cells were revived in buffer (DMEM/F12: Ham's F-12/DME High Glucose (GIBCO) supplemented with 2 mM L-glutamine, 100 units/ml Penicillin, 100 units/ml Streptomycin, 10\% fetal bovine serum (FBS) media as described above for COS-1 cells and grown to $90 \%$ confluence on a $10 \mathrm{~cm}$ dish. The day before the transfection, this plate was split 1:10 in DMEM buffer (DMEM-F12 supplemented with 100 units/ml P/S, 10\% FBS). On the second day (approx. 25 hours later) in a $15 \mathrm{ml}$ Falcon tube the following was mixed: $30 \mu \mathrm{g}$ of DNA, $450 \mu \mathrm{l}$ of water, $50 \mu \mathrm{l}$ of $2.5 \mathrm{M} \mathrm{CaCl}_{2}, 500 \mu 1$ of BES.

After the addition of BES, the mixture was incubated for exactly 1 minute prior to addition of the solution to the plate. After gentle distribution of the solution on the cells, plates were incubated at $35{ }^{\circ} \mathrm{C}, 3 \% \mathrm{CO}_{2}$ for 19 hours. After 1 hour the efficiency of the transfection was checked by light microscopy. If the transfection was successful, the DNA precipitate looked like fine sand between the cells and sometimes red crystals formed after longer incubation. However, large colorless crystals formed in between the cells during the overnight incubation, were indicative of a failed or inefficient transfection. After 19 hours incubation, the medium was removed and the plate was washed twice with $10 \mathrm{ml}$ of buffer (DMEM/F12: Ham's F-12/DME High Glucose (GIBCO) supplemented with $2 \mathrm{mM}$ L-glutamine, 100 units/ml Penicillin, 100 units/ml Streptomycin, 10\% FBS). The cells were then fed with the same buffer and the cells were returned to $37{ }^{\circ} \mathrm{C}, 5 \% \mathrm{CO}_{2}$. At this stage and for all further operations, the pipettes were changed for each mutant and plate thus as to prevent any contamination of different transfected mutants with each other. After further 23 hours, the plate was washed with PBS buffer, trypsinized and split 1:10. 6 plates for each transfected plate were prepared. To $1 \mathrm{ml}$ of $1 \mathrm{x}$ trypsin 
(0.05\%), $9 \mathrm{ml}$ of buffer (DMEM/F12: Ham's F-12/DME High Glucose (GIBCO) supplemented with $2 \mathrm{mM}$ L-glutamine, 100 units/ml Penicillin, 100 units/ml Streptomycin, 10\% FBS) was added and 10x pipetted up and down such that the cells did not clump. This was important for avoidance of mixed clones. $1 \mathrm{ml}$ of this was diluted with $9 \mathrm{ml}$ of PBS buffer. Of this diluted cell suspension, $40 \mu \mathrm{l}$ was mixed with $40 \mu \mathrm{l}$ of Trypan Blue. $10 \mu \mathrm{l}$ was pipetted under a glass plate to a hemacytometer. Approximately 2-5 single cells should be counted under the microscope, corresponding to $0.5-1 \times 106$ cells per plate. After further 20 hours, three of the plates were fed with buffer (DMEM/F12: Ham's F-12/DME High Glucose (GIBCO) supplemented with 2 mM L-glutamine, 100 units/ml Penicillin, 100 units/ml Streptomycin, 10\% FBS) media containing $2 \mathrm{mg} / \mathrm{ml}$ geneticin (G418) and three containing $3 \mathrm{mg} / \mathrm{ml}$ geneticin. Media were replaced every 23 days with fresh media until colonies formed and reached workable size (in about 2 weeks). The medium was aspirated from a plate where the colonies that were being picked had been circled. Per plate, 8 colonies could be picked. Cloning rings together with vacuum grease had been autoclaved. Using sterile forceps, the cloning rings were dipped in vacuum grease until the ring was completely covered from the bottom and were then placed on the colony. $40 \mu 1$ of $1 \mathrm{x}$ trypsin $(0.05 \%)$ was added to each of the 8 colonies in cloning rings. 1 minute after the addition of trypsin to the first cloning ring, $80 \mu \mathrm{l}$ of buffer (DMEM/F12: Ham's F-12/DME High Glucose (GIBCO) supplemented with $2 \mathrm{mM}$ L-glutamine, 100 units/ml Penicillin, $100 \mathrm{units} / \mathrm{ml}$ Streptomycin, 10\% FBS) media was added to each cloning ring in the same sequence as the trypsin had been added. Using a $200 \mu$ l eppendorf pipette, the solutions were pipetted up and down 5 times before being transferred to a 24 well plate containing $1 \mathrm{ml}$ of complete media with the appropriate amount of geneticin ( 2 or $3 \mathrm{mg} / \mathrm{ml}$, see above). As soon as confluence was reached, the cells were trypsinized and transferred into two chambers in a 6-well dish with a difference in cell number of 1:4. The 4 times higher concentrated chamber was used to perform western blot analysis.

Western blot positive clones were split to one $10 \mathrm{~cm}$ dish and one 6-well chamber. After feeding the plates on day 3 or 4, the $10 \mathrm{~cm}$ dish was grown until confluent and harvested with $10 \mathrm{ml}$ PBS buffer. Unlike COS-1 cell harvesting, HEK293 cell harvesting does not require scraping of the cells off the plates. Rather, cells were detached by pipetting. The cells were spun down for 10 minutes at $5000 \mathrm{rpm}$ at $4{ }^{\circ} \mathrm{C}$ in $15 \mathrm{ml}$ Falcon Tubes. Quantitation of the amount of rhodopsin was by difference spectroscopy as described below in Section 2.4.1. If the amount of 
rhodopsin per $10 \mathrm{~cm}$ dish was around $10 \mu \mathrm{g}$ or more the corresponding 6-well chamber was split to one $15 \mathrm{~cm}$ dish. Upon reaching confluence, this plate was split into 5 plates, three of which contained 2 or $3 \mathrm{mg} / \mathrm{ml}$ geneticin, while two only contained $0.5 \mathrm{mg} / \mathrm{ml}$ geneticin. The three first plates were used to generate freeze downs for long-term storage. One of the $0.5 \mathrm{mg} / \mathrm{ml}$ geneticin containing plate was split 1:5, while the other plate was fed until confluence was reached. This plate was used for quantitation of rhodopsin production as described for the $10 \mathrm{~cm}$ dish, except that $1 \mu \mathrm{l}$ of $10 \mathrm{mM} 11$-cis retinal was used per plate and the cell pellet was solubilized in $1 \mathrm{ml}$ of PBS buffer containing 1\% DM.

\subsubsection{Expression of rhodopsin in HEK293 cells}

Cells were kept frozen at $-70{ }^{\circ} \mathrm{C}$. Growing cells were obtained as described for COS-1 cells (Section 2.1.2(b)). After approximately 3 days the confluent cells were split typically 1:5. After removal of the medium, plates are washed with $25 \mathrm{ml}$ PBS buffer (15 cm dishes). $2 \mathrm{ml}$ of 0.05\% Trypsin-EDTA was added and the cells were left to detach from the plates. Cells were then transferred into the appropriate volume (25 $\mathrm{ml}$ medium per plate) of buffer (DMEM supplemented with $10 \%$ BS, 100 units $/ \mathrm{ml} \mathrm{P/S).} \mathrm{When} \mathrm{cells} \mathrm{were} \mathrm{about} 90 \%$ confluent, induction was performed with DMEM buffer with tetracycline and sodium butyrate (DMEM supplemented with $10 \% \mathrm{BS}, 100$ units/ml P/S, $2 \mu \mathrm{g} / \mathrm{ml}$ tetracycline, $5 \mathrm{mM}$ sodium butyrate. Note: When establishing stable cell lines FBS was used instead of BS medium. Cells were confluent after approximately 3 days for a 1:5 dilution.

Normally, stable cell lines were set up as suspension cultures in spinner flasks. However, since the mutants $\mathrm{P} 23 \mathrm{H}$ and $\mathrm{N} 15 \mathrm{~S}$ formed aggregates after induction, cells were grown in normal 10 or $15 \mathrm{~cm}$ plates, following the same protocol as COS-1 cells previously described 2.1.2(b).

\subsubsection{Reconstitution of the opsin expressed in mammalian cells with 11-cis retinal}

Opsin produced in COS-1 or HEK293 cells requires reconstitution with 11-cis retinal. Cells harvested from plates were resuspended in $2 \mathrm{ml}$ PBS buffer containing benzamidine $(0.005 \%)$ and PMSF $(0.7 \mathrm{mM})$ per plate. 11 -cis retinal was added to give $5 \mu \mathrm{M}$ concentration and the suspension was incubated at $4{ }^{\circ} \mathrm{C}$ for at least 1.5 hours. The concentration of reconstituted 
mutant rhodopsin was estimated by UV/Vis absorbance difference spectrum analysis of an aliquot solubilized in PBS buffer containing 1\% DM. 11-cis-Retinal was a gift from Dr. Rosalie Crouch, University of South Carolina, and the National Eye Institute of the National Institutes of Health, U. S. Public Health Services.

\subsubsection{Reconstitution of opsin expressed in mammalian cells with 9-cis retinal}

The procedure for reconstitution with 9-cis retinal was the same as described in Section 2.1.4 for 11-cis retinal. 9-cis retinal was purchased from Sigma-Aldrich (cat. number: R-5754).

\subsubsection{Reconstitution of opsin during synthesis in HEK293 cells with 9- and 11-cis retinal}

When confluent, cells were induced by adding tetracycline and sodium butyrate (DMEM supplemented with $10 \% \mathrm{BS}, 100$ units $/ \mathrm{ml} \mathrm{P/S,} 2 \mu \mathrm{g} / \mathrm{ml}$ tetracycline, $5 \mathrm{mM}$ sodium butyrate). After induction, 9-cis or 11-cis retinal was added directly to the tissue culture dish during synthesis of the protein to give a concentration of $20 \mu \mathrm{M}$ total, at 2 time points, 2 and 24 hours after induction (10 $\mu \mathrm{M}$ at each time point) (Krebs et al., 2010). Including and from this point on all steps were performed in the dark. Harvesting of the cells was done 48 hours after induction. Purification was performed as described in Sections 2.1.8(b) and 2.1.8(c).

\subsubsection{Solubilization of cell pellets containing rhodopsin using detergents}

For solubilization in DM, the rhodopsin containing cells or retinae were end-over-end mixed for 1-1.5 hours in buffer containing 1\% DM. HEK293 and COS-1 cells were solubilized at $2 \mathrm{ml}$ buffer/plate. Unsolubilized material was removed by centrifugation for 30 minutes at $4{ }^{\circ} \mathrm{C}$, 35,000 rpm, and rotor 70Ti Beckmann Ultra Centrifuge. The supernatant was added to 1D4Sepharose beads as described below in Section 2.1.8(b). 


\subsubsection{Affinity chromatography of rhodopsin using 1D4-sepharose}

\subsubsection{Preparation of 1D4-sepharose}

$\mathrm{CNBr}$-activated sepharose 4B was prepared from $\mathrm{CNBr}$ and sepharose 4B (Kumel et al., 1979), based on the rapid reaction of cyanogen halides with the hydroxyl groups of carbohydrates at high $\mathrm{pH}$ to form cyanate esters (Figure 2.2). The monoclonal anti-rhodopsin antibody 1D4 (Oprian et al., 1987) was then coupled to $\mathrm{CNBr}$-activated sepharose 4B via its amino groups (Oprian et al., 1987) with minor modifications.

Figure 2.2 - Activation of sepharose by $\mathrm{CNBr}$ and coupling of protein to the activated gel.

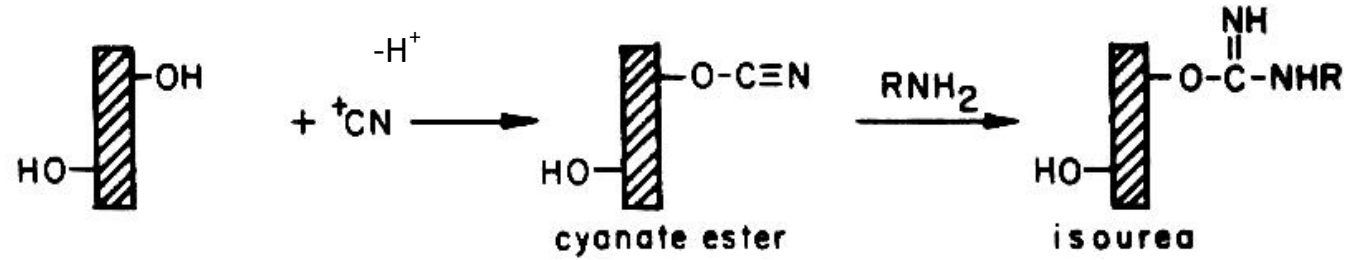

SOURCE: Figure is taken from (Kohn and Wilchek, 1978). With permission.

Prior to coupling, 1D4 was purified from a myeloma cell line provided by R.S. Molday (University of British Columbia) as follows. $1500 \mathrm{mg}$ of 1D4 antibody (stored at $-20{ }^{\circ} \mathrm{C}$ ) were thawed. $300 \mathrm{ml}$ of Protein A-sepharose 4B were packed and equilibrated with 5 times the bed volume of buffer $(1.5 \mathrm{M}$ glycine, $3 \mathrm{M} \mathrm{NaCl})$. All flow rates were between 0.5-1.5 ml/minute, unless otherwise stated. The sample was diluted with 5 times its original volume of buffer $(1.5 \mathrm{M}$ glycine, $3 \mathrm{M} \mathrm{NaCl})$. The sample was loaded, followed by washing with buffer (1.5 M glycine, 3 $\mathrm{M} \mathrm{NaCl}$ ) until a straight baseline at $\mathrm{A}_{280}$ was reached. The sample was eluted with approximately $300 \mathrm{ml}$ of buffer $(0.1 \mathrm{M}$ citric acid, $0.15 \mathrm{M} \mathrm{NaCl}(\mathrm{pH} 3))$. Fractions of $4 \mathrm{ml}$ each were collected while monitoring $\mathrm{A}_{280}$. In order to store the column for future use, it was regenerated with $300 \mathrm{ml}$ buffer $\left(6 \mathrm{M}\right.$ guanidinium- $\mathrm{HCl}$ ) at a flow rate of $0.5 \mathrm{ml} /$ minute. The column was stored at $4{ }^{\circ} \mathrm{C}$ after washing with 2-10 times the bed-volume of buffer (50 mM Na $2 \mathrm{HPO}_{4} / \mathrm{NaH}_{2} \mathrm{PO}_{4}(\mathrm{pH} 7)$, $0.01 \%$ thimerosal). Due to the poor stability of $1 \mathrm{D} 4$ at $\mathrm{pH} 3$, the $\mathrm{pH}$ of the fractions was immediately after elution adjusted to $\mathrm{pH} 8.3$ using $0.5 \mathrm{M} \mathrm{NaHCO}_{3} \mathrm{pH} 10$. Fractions containing 1D4 sepharose were combined and used directly for coupling, as long as the concentration of 1D4 was above $3 \mathrm{mg} / \mathrm{ml}$. If the concentration was lower, the solution was first concentrated to up 
to $10 \mathrm{mg} / \mathrm{ml}$ before coupling. The concentration of antibody was determined using $\mathrm{A}_{280}\left(\mathrm{~A}_{280}=\right.$ 1.383 is equal to a concentration of $1 \mathrm{mg} / \mathrm{ml}$ ). Dialysis tubes (Spectrapor, $14 \mathrm{kD}$ cut-off, but could be higher) were washed thoroughly with aqua distillate and filled with the 1D4 solutions. Dialysis was against 5-10 liters of buffer $\left(0.25 \mathrm{M} \mathrm{NaHCO}_{3}, 0.5 \mathrm{M} \mathrm{NaCl}(\mathrm{pH} 8.3)\right)$ for 4 hours to overnight. Dialysis buffer was subsequently changed at least 3 times. After dialysis, the concentration was determined using $\mathrm{A}_{280}$ as above.

The purified 1D4 antibody was coupled to $\mathrm{CNBr}$ sepharose as described below. First, $20 \mathrm{ml} \mathrm{CNBr-Sepharose} \mathrm{was} \mathrm{prepared.} \mathrm{100-200} \mathrm{mg} \mathrm{of} \mathrm{1D4} \mathrm{antibody} \mathrm{can} \mathrm{be} \mathrm{bound} \mathrm{to} \mathrm{this} \mathrm{amount}$ of CNBr-Sepharose. If $100 \mathrm{mg}$ of 1D4 was coupled the capacity of the final product was typically $1 \mathrm{mg}$ rhodopsin/ml of 1D4 sepharose. $500 \mu \mathrm{l}$ acetonitrile was added per $1 \mathrm{~g}$ of BrCN (Sigma). The volume increased after dissolving the $\mathrm{BrCN}$, so that the final concentration was $1 \mathrm{~g} / \mathrm{ml}$. This stock solution could be stored at $-20{ }^{\circ} \mathrm{C}$. Sepharose 4B slurry (20 g) (Sigma) was washed three times with water in a filter funnel. Where, $20 \mathrm{~g}$ of sepharose corresponds to $30 \mathrm{ml}$ of slurry. Water was removed by vacuum and the sepharose was added to $30 \mathrm{ml}$ of buffer $\left(3.3 \mathrm{M} \mathrm{K}_{3} \mathrm{PO}_{4}\right.$ ( $\mathrm{pH}$ of 11.9 at 10 -fold dilution)). While stirring vigorously with a regular stirring bar under the hood, $1 \mathrm{ml}$ of BrCN-solution was added and stirred for exactly 2 minutes. The suspension was transferred quickly to the filter funnel that contained ice-cold water and was vacuum-filtered immediately. The beads were washed with $300 \mathrm{ml}$ of ice-cold buffer $\left(0.25 \mathrm{M} \mathrm{NaH} \mathrm{PO}_{4} / \mathrm{Na}_{2} \mathrm{HPO}_{4}\right.$ (pH 6)) followed by $500 \mathrm{ml} 1 \mathrm{mM} \mathrm{HCl}$. All the washes were carried out extremely rapidly, i.e. such that vacuum-filtering of $100 \mathrm{ml}$ took less than 30 seconds. Thus prepared CNBr-sepharose could not be stored. Coupling to 1D4 had to follow immediately after preparation, ideally within 2 minutes. If CNBr-sepharose was purchased from Pharmacia, $1 \mathrm{~g}$ of the dry powder $\mathrm{CNBr}$ sepharose was equivalent to approx. $3.5 \mathrm{ml}$ gel slurry. The dry $\mathrm{CNBr}$ sepharose was washed on a sintered glass filter with $500 \mathrm{ml}$ of $1 \mathrm{mM} \mathrm{HCl}$. All subsequent steps were the same for commercially available or self-made $\mathrm{CNBr}$ sepharose.

The coupling to $1 \mathrm{D} 4$ to $\mathrm{CNBr}$ sepharose was carried out at 3-10 mg 1D4 per $1 \mathrm{ml} \mathrm{CNBr-}$ sepharose 4B. Slurry $(20 \mathrm{ml})$ was added to the concentrated sample of 1D4 containing $100 \mathrm{mg}$ $1 \mathrm{D} 4$ in buffer (0.25 $\left.\mathrm{M} \mathrm{NaHCO}_{3}, 0.5 \mathrm{M} \mathrm{NaCl}(\mathrm{pH} 8.3)\right)$. Coupling was allowed to proceed by endover-end mixing at until the supernatant after spinning down the beads for 5 minutes contained less than 5\% of the total protein (after 4-5 hours at RT). The supernatant was discarded. A volume equal to that of the original supernatant of blocking agent (1 M ethanolamine $\mathrm{pH} 8)$ was 
added. Blocking was allowed to proceed for 2 hours at RT or overnight at $4{ }^{\circ} \mathrm{C}$. The beads were washed on a sintered glass filter 4 times with alternating solutions of buffer $\left(0.25 \mathrm{M} \mathrm{NaHCO}_{3}\right.$, $0.5 \mathrm{M} \mathrm{NaCl}(\mathrm{pH} \mathrm{8.3)})$ and buffer (0.1 M NaOAc, $0.5 \mathrm{M} \mathrm{NaCl}(\mathrm{pH} 4))$. PBS buffer containing $0.05 \% \mathrm{NaN}_{3}$ was added in equal volume as the beads. The coupled 1D4-sepharose was stored at $4{ }^{\circ} \mathrm{C}$.

The capacity of 1D4-Sepharose was determined as follows. $500 \mu \mathrm{g}$ ROS membranes were solubilized using DM as described in Section 2.1.1 and the exact concentration was determined spectroscopically (as described, Section 2.3.1). $200 \mu \mathrm{l}$ of 1D4-sepharose were added and rhodopsin was purified as described in 2.1.8(b) and 2.1.8(c). The capacity was calculated using the ratio between the rhodopsin purified and the amount of rhodopsin originally solubilized. Typically the capacity was $1 \mathrm{mg}$ rhodopsin per $1 \mathrm{mg}$ 1D4-sepharose if $100 \mathrm{mg}$ of $1 \mathrm{D} 4$ was coupled to $20 \mathrm{ml}$ of $\mathrm{CNBr}$-sepharose. A capacity of up to $1.7 \mathrm{mg} / \mathrm{ml}$ could be obtained if correspondingly larger amounts of 1D4 were used.

\subsubsection{Binding of solubilized rhodopsin to 1D4-sepharose}

After centrifugation of non-solubilized material (Section 2.1.1 above), the supernatant was added to 1D4-Sepharose. The amount of beads necessary to bind quantitatively the rhodopsin present in the supernate could be calculated from the 1D4 sepharose binding capacity, usually $1 \mathrm{mg}$ of rhodopsin per ml settled beads. About $10 \%$ excess of 1D4-Sepharose over rhodopsin content was used. After end-over-end mixing for at least 6 hours at $4{ }^{\circ} \mathrm{C}$, the suspension was packed into a column. 100-500 $\mu \mathrm{g}$ rhodopsin amounts were purified using BIORAD disposable chromatographic columns. The packed beads were washed at RT with at least 50 column volumes of PBS buffer with $0.05 \%$ DM. This was followed by further washes with 10 bed volumes of $2 \mathrm{mM}$ sodium phosphate $\mathrm{pH} 6$ with $0.05 \%$ DM. The flow rate was 0.5 $1 \mathrm{ml} /$ minute. 


\subsubsection{Elution of rhodopsin from 1D4-sepharose}

Rhodopsin was eluted from 1D4-Sepharose using buffer $2 \mathrm{mM} \mathrm{Na} \mathrm{HPO}_{4} / \mathrm{NaH}_{2} \mathrm{PO}_{4}$ (pH 6) containing $70 \mu \mathrm{M}$ epitope nonapeptide at a flow rate of $0.3-0.35 \mathrm{ml} / \mathrm{minute}$, the effluent being monitored by UV/Vis absorption spectroscopy. Complete elution of the rhodopsin mutants usually required about 5 column volumes of elution buffer. The elutions, where the $\mathrm{A}_{280} / \mathrm{A}_{500}$ ratio was within the range $1.6-2.2$, were considered for further studies ensuring proper folding of rhodopsin.

\subsection{ANIMAL PROCEDURES}

All experiments were conducted in accordance with the ARVO Statement on the Use of Animals in Ophthalmic and Vision Research. P23H (line 3) and S334ter (line 4) homozygous animals for breeding were kindly provided by Matthew LaVail, PhD (UCSF School of Medicine, Beckman Vision Center). Homozygous animals carry two copies of the mutant transgene. Experimental rats should carry only one copy of the mutant transgene. To produce rats for experiments, the homozygous rats were bred against normal Sprague-Dawley (SD) rats, producing litters in which all the animals are affected, but carrying only one copy of the mutant transgene (hemizygotes) in addition to the normal two WT opsin alleles. Lines 3 and 4 of P23H and S334ter, respectively, were chosen because they present a similar pattern and degree of retinal degeneration over time (http://www.ucsfeye.net/mlavailRDratmodels.shtml). The retinal degeneration of these lines is already significant at the age of 8 weeks, which allows for an easy comparison between different experimental treatments at early or later stages.

\subsubsection{Breeding and maintenance of WT rats, homozygous $\mathrm{P} 23 \mathrm{H}$ and S334ter rats}

WT SD, P23H and S334ter rats were bred, born and reared at the animal house facility of the Department of Cell and Developmental Biology, at the University of São Paulo, were they were kept on a $12 / 12$ hours light/dark cycle at $25{ }^{\circ} \mathrm{C}$ with food and water ad libitum, until they

reached the correct age for experimental use. Adult $\mathrm{P} 23 \mathrm{H}$ and $\mathrm{S} 334$ ter rats were used for 
breeding against adult albino SD at the time of experimental use. During and after treatment, rats were maintained at same conditions, unless otherwise stated.

2.2.2 Light-induced degeneration after treatment with DMTU and chlorin e6 (Ce6)

Two month old SD rats were intraperitoneally injected with DMTU (500 mg/kg) or Ce6 (2, 10 or $20 \mathrm{mg} / \mathrm{kg}$ ), 24 and 1 hour before exposure to light. Control rats were not injected. Rats were dark-adapted for 16 hours before exposure to constant light in a reflective cage. The pupils of the rats were dilated with 5\% cyclopentolate (Allergan, São Paulo, Brazil) immediately before stimulation.

Rats were then exposed to a cool white fluorescent light of 5000 lux (TLD 36W, OSRAM), intensity of radiation of $0.01 \mathrm{Watts} / \mathrm{cm}^{2}$, for 6 hours inside the cage that was covered with glass. Light exposure started at 10am.

After light exposure, the rats were returned to dim cyclic light that consisted of 12/12 hours dark/light cycle for 7 days. At this point, rats were sacrificed and the eyes were removed for retinal analysis.

\subsubsection{Long-time treatment with Ce6}

Ce6, $2 \mathrm{mg} / \mathrm{kg}$, was administered by intraperitoneal injections (Washington et al., 2007) every second day for $\mathrm{P} 23 \mathrm{H}$ and S334ter rats. Controls $\mathrm{P} 23 \mathrm{H}$ and S334ter rats were treated only with $0.1 \mathrm{M}$ phosphate buffer $\mathrm{pH} 7.4$ (a master solution of $0.2 \mathrm{M}$ phosphate buffer was initially prepared: $21.8 \mathrm{~g} \mathrm{Na}_{2} \mathrm{HPO}_{4}, 6.4 \mathrm{~g} \mathrm{NaH}_{2} \mathrm{PO}_{4}$ in $1000 \mathrm{ml} \mathrm{H} 2 \mathrm{O}$. From this solution, dilutions were made accordingly with the protocol used). Treatment started at postnatal day 20 (P20) and animals were sacrificed at P60, P90 and P150. Control animals were sacrificed at the same time points. 


\subsubsection{Extraction and fixation of rat eyes}

Rats were given deep anesthesia by intramuscular injection of ketamine $(80-100 \mathrm{mg} / \mathrm{Kg})$ and xylazine $(5-10 \mathrm{mg} / \mathrm{kg}$ ) and transcardial perfusion was performed with $0.9 \% \mathrm{NaCl}$ in $0.1 \mathrm{M}$ phosphate buffer followed by fixation with a solution of $2 \%$ paraformaldehyde (PFA) in $0.1 \mathrm{M}$ phosphate buffer $\mathrm{pH}$ 7.4. For preparation of $100 \mathrm{ml}$ of $2 \%$ PFA, $2 \mathrm{~g}$ of PFA was added to $48 \mathrm{ml}$ $\mathrm{H}_{2} \mathrm{O}$, heated to dissolve. $\mathrm{NaOH}$ was added until the solution was clear. Then, $50 \mathrm{ml}$ of $0.2 \mathrm{M}$ phosphate buffer $\mathrm{pH} 7.4$ was added. The solution was filtered and kept on ice. Eyes were enucleated and kept for 15 minutes in $2 \%$ PFA in $0.1 \mathrm{M}$ phosphate buffer $\mathrm{pH} 7.4$ and cryoprotected in $30 \%$ sucrose in $0.1 \mathrm{M}$ phosphate buffer $\mathrm{pH} 7.4$ for at least 24 hours at $4{ }^{\circ} \mathrm{C}$. Cornea, lens and vitreous humor were then removed and the eyes were embedded in OCT (optimum cutting temperature) compound (Compound Sakura Finetek CRYO-OCT).

\subsubsection{Sectioning, storage and processing of rat eyes}

Retinas were sectioned perpendicularly to the vitreal surface in a cryostat $(12 \mu \mathrm{m}$ thick slices). Samples of temporal, central and nasal areas were collected and stored at $-20{ }^{\circ} \mathrm{C}$. The total number of glass slides stored was 10-12 per rat, each containing samples of 6 regions of the eye from nasal to temporal area. Figure 2.3 shows a schematic drawing of a glass slide where the collected samples are stored. The different areas of the eye are also schematically displayed.

Figure 2.3 - Schematic diagram of a glass slide containing the retinal sections.

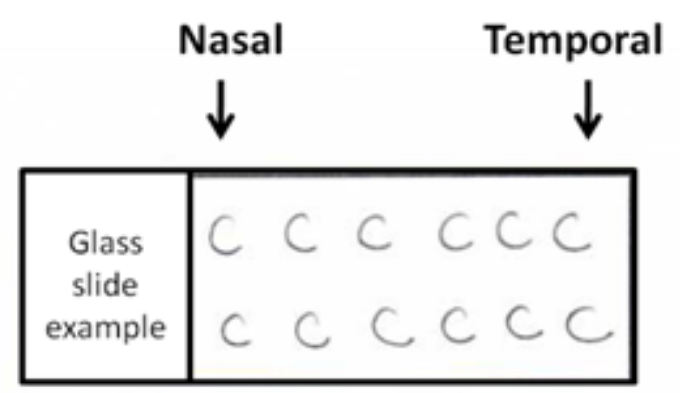

Nasal and temporal areas are marked. Nasal refers to the area of the eye that is closest to the nose and temporal refers to the opposite side, closest to the ear. Sections in the middle represent sampling of the whole eye between nasal and temporal areas. Samples of both right and left eyes were collected.

SOURCE: Balen, 2012 
Tissue sections were then prepared for immunohistochemistry. Samples were taken out from the freezer and the excess of OCT that was around the slices was cleaned using a swab and marked with a wax pen (Liquid Blocker, Super Pap Pen, Daido Sangyo, Japan) to delineate the desired sections and dried in a hot plate for 45 minutes at $37^{\circ} \mathrm{C}$. Sections were then washed 3 times with $0.1 \mathrm{M}$ phosphate buffer $\mathrm{pH} 7.4$ and blocked with blocking buffer $(0.3 \%$ Triton $\mathrm{X}$ $100,3 \%$ normal donkey serum (NDS), $1 \%$ BSA in $0.1 \mathrm{M}$ phosphate buffer $\mathrm{pH} 7.4$ ). Incubation with primary antibodies (rabbit anti-PKC 1:750 (Sigma, P4334), rabbit anti-synaptophysin 1:250 (Dako Ctomation, A0010), goat anti-vimentin 1:250 (Chemicon, AB1620), mouse anti-calbindin 1:500 (Sigma, D-28K, clone CB 955), and anti-1D4 1:40000) overnight, followed by secondary antibodies (donkey anti rabbit FITC or TRICT, Jackson Labs and Alexa 488, Molecular probes) for 2 hours was done in a humidified plate at room temperature. Sections were washed 3 times with $0.1 \mathrm{M}$ phosphate buffer $\mathrm{pH} 7.4$ and incubated with nucleus marker, Propidium Iodide (red) or DAPI (4',6-diamidino-2-phenylindole, blue), dilution 1:1000 for 10 minutes. After washing, sections were mounted with Vecta Shield (Vector Labs).

\subsection{SPECTROSCOPIC AND MICROSCOPIC TECHNIQUES}

\subsubsection{Absorbance Spectroscopy}

A Perkin-Elmer $\lambda$-25 spectrophotometer, equipped with a multiple water-jacketed cuvette holder was used to record absorption spectra. All spectra were measured at $20{ }^{\circ} \mathrm{C}$ unless otherwise specified. All measurements were recorded in the $250 \mathrm{~nm}$ to $650 \mathrm{~nm}$ range, using a $10 \mathrm{~mm}$ path length cell, bandwidth of $1 \mathrm{~nm}$, with a response time of $1 \mathrm{~s}$ and scan speed of $960 \mathrm{~nm} /$ minute.

Rhodopsin concentrations were calculated using a molar extinction coefficient for the $500 \mathrm{~nm}$ chromophore absorption of 40,600 $\mathrm{M}^{-1} \mathrm{~cm}^{-1}$ (Wald and Brown, 1953). A 500 was determined either directly from absorbance spectra of pure rhodopsin solutions, or for solutions with a high absorbance background, i.e. solubilized membranes, by absorbance difference spectroscopy. First, the dark spectrum was recorded. The rhodopsin solution was illuminated and

the spectrum was again recorded. The difference between the two spectra allowed estimation of $\mathrm{A}_{500}$ and the concentration of rhodopsin was calculated. 
For light-activating rhodopsin, the samples were illuminated with a $150-\mathrm{W}$ fiber optic light (Fiber Lite A-200; Dolan-Jenner, Woburn, MA) equipped with a > $495 \mathrm{~nm}$ long-pass filter for 30 seconds.

Thermal stability experiments were performed either at $50{ }^{\circ} \mathrm{C}$ or $55^{\circ} \mathrm{C}$. Both sample and buffer were placed in the cuvette holders and the first data point was obtained at $20{ }^{\circ} \mathrm{C}$ (room temperature). Samples were then taken out and kept on ice until the temperature of the machine, which is equipped with a water circulated temperature control (water jacket), reached the desired final temperature, $50{ }^{\circ} \mathrm{C}$ or $55^{\circ} \mathrm{C}$. To assure the temperature, an external thermometer was used. For that, a second pair of cuvettes filled with water was placed on the cuvettes holder, and the temperature was manually verified until it reached the desired temperature and was stabilized.

\subsubsection{Fluorescence Spectroscopy}

Tryptophan fluorescence of rhodopsin was recorded using a Varian Cary Eclipse instrument (Varian Inc., Palo Alto, CA). In all experiments, the data acquisition parameters were similar to those described previously (Farrens and Khorana, 1995): excitation and emission wavelengths were at $295 \mathrm{~nm}$ and $330 \mathrm{~nm}$, with slit widths of $5 \mathrm{~nm}$ and $10 \mathrm{~nm}$ or $5 \mathrm{~nm}$ and $5 \mathrm{~nm}$,

as described in the figures, respectively. Samples contained $0.25 \mu \mathrm{M}$ or $0.5 \mu \mathrm{M}$ (as indicated) of purified rhodopsin in $0.05 \% \mathrm{DM}$, either in the absence or presence of additives and the fluorescence were measured in the dark and after illumination. The sample temperature was kept at $20{ }^{\circ} \mathrm{C}$ unless otherwise specified.

For thermal denaturation experiments, the temperature was raised to $55{ }^{\circ} \mathrm{C}$ and changes in fluorescence were measured over time at this temperature.

\subsubsection{Circular Dichroism Spectroscopy}

CD spectra were acquired using a JASCO J-810 spectropolarimeter. All spectra were recorded from $195 \mathrm{~nm}$ to $260 \mathrm{~nm}$, using a bandwidth of $1 \mathrm{~nm}$, scan speed of $100 \mathrm{~nm} /$ minute, time constant of 1 second. An average of ten scans was acquired for each spectrum. Reference spectra were subtracted from the subsequently recorded samples containing protein. The presence of nonapeptide in rhodopsin samples was not corrected as its contribution to the ellipticity at $222 \mathrm{~nm}$ 
was estimated to be less than 5\%. The thermal denaturation of rhodopsin in the presence of metal ions and $\mathrm{Ce} 6$ were recorded by increasing the temperature from $5{ }^{\circ} \mathrm{C}$ to $100{ }^{\circ} \mathrm{C}$, with intervals of $5{ }^{\circ} \mathrm{C}$, taking around 4 hours for the whole experiment to be complete. Additionally, the spectra recorded for rhodopsin without and with $\mathrm{Zn}^{2+}$ and $\mathrm{Ce} 6$ were subsequently decreased from $100{ }^{\circ} \mathrm{C}$ to $5{ }^{\circ} \mathrm{C}$ to test for reversibility. Rhodopsin, $\mathrm{Ce} 6$ and divalent metal ion concentrations used were $2.5 \mu \mathrm{M}, 100 \mu \mathrm{M}$ and $100 \mu \mathrm{M}$, respectively. The samples were placed in a $1 \mathrm{~mm}$ quartz cell (Hellma Analytics, type 110-QS). Qualitative estimates of helix content were obtained from the CD spectra by spectral deconvolution using CDPro software (Sreerama and Woody, 2000). Protein reference set 10, which includes membrane proteins, was used.

\subsubsection{Total Reflectance Fourier Transform Infrared (ATR/FT-IR) spectroscopy}

Samples were measured at the University of Bochum, in the laboratory of Professor Klaus Gerwert, using a FTIR spectrometer. A trapezoid germanium internal reflection element (IRE) was used in a vertical, variable angle ATR setup (Specac), in a Bruker IFS 66 FTIR spectrometer equipped with a liquid nitrogen cooled mercury cadmium telluride (MCT) detector, as described elsewhere (Ollesch et al., 2007). The IRE was cleaned with sulfuric acid, rinsed thoroughly with distilled water. The IRE was held in a customized, streamoptimized flow-through cuvette with non-grease silicone sealing. The protein solution was introduced into the system and excess protein was rinsed out with excess amounts of buffer. The spectra were continuously recorded prior to Fourier transformation.

To estimate protein secondary structures, a calibrated amide I band decomposition was performed (Ollesch et al., 2007). Briefly, amide I bands of WT, N15S and P23H spectra recorded using PBS buffer plus $0.05 \%$ DM either in $\mathrm{H}_{2} \mathrm{O}$ or $\mathrm{D}_{2} \mathrm{O}$ were decomposed into a set of Cauchy (a symmetric and bell shaped distribution) curves. The decomposition was carried out to obtain a resulting set that showed (i) a high quality band decomposition for all bands and (ii) a minimum deviation of the secondary structure fractions from the calibration data. The initialization parameters needed as input for the decomposition were generated based on the X-ray crystal structure of rhodopsin (PDB ID: 1L9H) using STRIDE secondary structure analysis (Frishman and Argos, 1995). Missing residues in the PDB file were assigned to be in random coil conformation. The relative contents of different secondary structure fractions were estimated as 
the ratio between the band integrals corresponding to specific secondary structure fractions and the whole area of the amide I band region.

\subsubsection{Confocal Microscopy}

\subsubsection{Collagen coating coverslips}

Coverslips were immersed individually in $90 \%$ ethanol and then air-dried in a culture dish for a few minutes. Enough $(200 \mu \mathrm{l} /$ coverslip) collagen $(0.3 \mathrm{mg} / \mathrm{ml}$ dissolved in water; BD Biosciences, catalogue number: 354259) was added to cover the surface of the coverslips and left for 1 hour. After 1 hour, the collagen was aspirated and kept to dry overnight under ultraviolet light.

\subsubsection{Growing and preparing cells on coverslips for imaging}

A confluent $150 \mathrm{~mm}$ cell culture plate of COS-1 cells or HEK293S stable cell lines was split 1:10 and the cells were added to a $150 \mathrm{~mm}$ cell culture dish with collagen coated coverslips in it. The cells are usually 50-60\% confluent 32-48 hours after plating. At this time, transfection was carried out in the case of COS-1 cells using the DEAE-transfection method (Oprian et al., 1987) as described in Section 2.1.2.2. The HEK293S stable cells were induced as described in Section 2.1.3.3 (Chen and Okayama, 1987; O'Mahoney and Adams, 1994). Immunostaining of the cells was carried out 48-55 hours post-transfection or 48 post-induction, respectively.

\subsubsection{Immunostaining}

A Leica TCS SL and Olympus Fluoview 1000 (inverted) confocal fluorescence microscope at the Centre of Biological Imaging (CBI) at the University of Pittsburgh (http://www.cbi.pitt.edu/) were used for imaging the cells. To prepare cells for imaging, a confluent $150 \mathrm{~mm}$ cell culture plate was split 1:10 and the cells were added to a $150 \mathrm{~mm}$ plate or 24 well cell culture dish containing collagen coated coverslips. The cells were induced when they were 50-60\% confluent (32-48 hours after plating). Immunostaining of coverslips was carried out 
48-55 hours post transfection or induction depending on the system chosen. The coverslips with cells were carefully transferred to a 24 well plate with PBS, when necessary. PBS in the wells was aspirated and the wells were washed 3 times with $1 \mathrm{ml}$ PBS. To fix the cells, 2\% paraformaldehyde in PBS was added to each well $(500 \mu 1 /$ well $)$, which were then incubated for 15 minutes. The cells were then permeabilized with $0.1 \%$ Triton $\mathrm{X}$ in PBS for 15 minutes and rehydrated with three $1 \mathrm{ml}$ washes with PBS. Cells were then washed five times with $0.5 \%$ BSA in PBS and then blocked with 2\% BSA in PBS for 45 minutes. Cells were washed again five times with $0.5 \%$ BSA in PBS. The cells were then incubated with primary antibody prepared as a 1:1000 dilution of $2 \mathrm{mg} / \mathrm{ml}$ 1D4 antibody in 0.5\% BSA in PBS for 60 minutes. 1D4 antibody was obtained from Dr. Robert Molday of the University of British Columbia, Vancouver, BC, Canada. After incubation, the cells were washed 5 times with $0.5 \%$ BSA in PBS. $500 \mu 1 /$ well of $0.5 \% \mathrm{BSA}$ in PBS containing 1:500 dilution of $2 \mathrm{mg} / \mathrm{ml}$ Alexa fluor 488 goat anti-mouse secondary antibody (Invitrogen, A11029), 1:250 dilution of $\sim 6.6 \mu \mathrm{M}$ Rhodamine-Phalloidin (Invitrogen, R415) and 1:1000 dilution of $5 \mathrm{mM}$ DRAQ-5 (Biostatus, DR50201) were added. After 60 minutes incubation, the cells were washed five times with 0.5\% BSA in PBS, followed by another five washes with PBS. The coverslips were then mounted on clear glass slides. A small drop of gelvatol (obtained from CBI, University of Pittsburgh) was applied on a clean slide and the coverslips were placed with cells facing down on the gelvatol drop. Excess gelvatol was cleaned on the sides with a tissue paper. Cover slips were dried overnight at $4{ }^{\circ} \mathrm{C}$ in the dark before taking images.

\subsubsection{Electroretinography}

For ERG measurements, the animals were dark adapted overnight and all subsequent preparation was carried out under dim red light illumination. Animals were anaesthetized intraperitoneally with $80-100 \mathrm{mg} / \mathrm{kg}$ ketamine and 5-10 mg/kg xylazine. The pupils were dilated with a drop of tropicamida (Tropinom, Laboratory Ophthalmos, São Paulo) followed by a drop of methylcellulose (Laboratory Ophthalmos, São Paulo) to prevent the drying of the eyes. Three electrodes were placed on the animal; a Gold Ring electrode on the cornea of the eye and two needle electrodes were placed under the skin of the head and tail for reference and ground, respectively. 
For ERG measurements, the animals were placed in a Faraday cage $(60 \times 60 \mathrm{~cm})$. The rat was positioned lying on its front, looking down, on top of a tupperware containing warm water in order to maintain the normal body temperature. The light stimuli were generated by a GRASS stimulator (PS33 PLUS - Photic Flash Visual Stimulator with a flash lamp bulb - model PST2100, Grass Technologies), and presented by a Ganzfeld (LKC 25038) situated inside the Faraway cage, which was fully controlled by a computer. ERG responses were recorded from one eye that was randomly chosen by coin flipping. The ERG signals were amplified and digitized (NI-DAQ, National Instruments) and analyzed by the data acquisition software (MONODAQ), donated by Steve Nusinowitz and John Ramirez, University of California.

The International Society for Clinical Electrophysiology of Vision (ISCEV) protocol (Harazny et al., 2009) was followed. Measurements consisted of 3 parts:

1. Scotopic response: light emission consisted of 10 flashes at 5 different intensities: 0.003; $0.03 ; 0.3 ; 3$ and $30 \mathrm{~cd} \cdot \mathrm{s} / \mathrm{m}^{2}$ (luminance is produced by a xenon-light source). Intervals between flashes were 5, 8, 10, 13 and 15 seconds, respectively.

2. Cone response: following the scotopic response recording, animals were light adapted for 2 minutes $\left(30 \mathrm{~cd} \cdot \mathrm{s} / \mathrm{m}^{2}\right)$. Under this background light, 10 flashes at $30 \mathrm{~cd} \cdot \mathrm{s} / \mathrm{m}^{2}$ were emitted with inter-flash intervals of 5 seconds.

3. Flicker response: just after the cone response recording, 50 flashes at $30 \mathrm{~cd} \cdot \mathrm{s} / \mathrm{m}^{2}$ were emitted on the $30 \mathrm{~cd} / \mathrm{m}^{2}$ background at 12, 18, 24 and $30 \mathrm{~Hz}$.

\subsubsection{Fluorescence Microscopy}

Retinal sections were inspected visually and images were obtained by using a Nikon E100 fluorescent microscope with a digital camera attached or a Nikon PCM2000 confocal microscope. Figures were prepared with Adobe Photoshop CS5.1 (Deneba), and measurements were performed off-line using the Image $\mathbf{J}$ program (National Institute of Health, USA). Manipulation of the images was restricted to threshold and brightness adjustments to the whole image. 


\subsection{ANALYTICAL PROCEDURES}

\subsubsection{Protein quantification}

Protein concentrations were determined using the Bradford assay (BIO-RAD Protein Assay cat. num. 500-0006). A calibration curve was obtained by mixing 0, 10, 20, 30, 40 and $50 \mu \mathrm{l}$ of a $0.1 \mathrm{mg} / \mathrm{ml} \mathrm{BSA} \mathrm{stock} \mathrm{solution} \mathrm{with} \mathrm{water} \mathrm{such} \mathrm{that} \mathrm{the} \mathrm{final} \mathrm{volume} \mathrm{was} 100 \mu 1$. The sample solution was also adjusted to $100 \mu \mathrm{l}$ at appropriate dilutions. The Bradford reagent (BIORAD) was diluted 5-fold and $1 \mathrm{ml}$ was added to each Eppendorf tube. After mixing and incubation for 5 minutes, the $595 \mathrm{~nm}$ absorbance was measured in a disposable plastic cuvette. The concentration was determined using the bovine serum albumin standard curve.

\subsubsection{Meta II decay}

The rate of Meta II decay was measured, after illumination of the samples by following the rate of fluorescence increase, which corresponds to the rate of retinal release (Farrens and Khorana, 1995). The samples were bleached at $20{ }^{\circ} \mathrm{C}$ for 30 seconds and the fluorescence increase was measured. The excitation and emission wavelengths were $295 \mathrm{~nm}$ (slit width = $5 \mathrm{~nm}$ ) and $330 \mathrm{~nm}$ (slit width $=10 \mathrm{~nm}$ ), respectively. Purified rhodopsin $0.25 \mu \mathrm{M}-0.5 \mu \mathrm{M}$ in $0.05 \% \mathrm{DM}$ and $2 \mathrm{mM}$ sodium phosphate buffer at $\mathrm{pH} 6$ were used. The concentration of Ce6 was varied as indicated in the graphs.

For the Meta II decay studies of rhodopsin in presence of metal ions, a concentration of $0.5 \mu \mathrm{M}$ and $25 \mu \mathrm{M}$ for rhodopsin and metal ions, respectively. Samples were prepared in the dark

prior to placing them in the fluorimeter. Around 30 seconds after the samples were placed in the machine (equilibration time), the experiment was initiated. 


\subsubsection{Binding of small molecules monitored by fluorescence spectroscopy}

Binding of either $\mathrm{C} 3 \mathrm{G}$, Ce6 or metal ions was quantified by measuring the fluorescence of $0.5 \mu \mathrm{M}$ of rhodopsin in $500 \mu \mathrm{l}$ of $2 \mathrm{mM}$ sodium phosphate $\mathrm{pH} 6,0.05 \% \mathrm{DM}$ as described (Farrens and Khorana, 1995). C3G and Ce6 were added prior to illumination or temperature increase from a $100 \mathrm{mM}$ DMSO stock solution or its dilutions, at the final concentrations indicated. The difference in total fluorescence between rhodopsin in the absence and presence of various concentrations of ligands was used to estimate the affinity of interaction.

For the studies of metal ions, $\mathrm{Ce} 6$ and both combined, the molecules were added to the rhodopsin samples prior to illumination or increasing the temperature in the dark, from stocks of $100 \mathrm{mM}$ in water for metal ions and $100 \mathrm{mM}$ DMSO stock for Ce6, or its dilution as required.

For all the experiments that required illumination or temperature variation a first data point was collected at $20^{\circ} \mathrm{C}$ and then the experiment was continued.

\subsubsection{Thermal denaturation}

Thermal stability experiments using absorbance spectroscopy were performed at $50{ }^{\circ} \mathrm{C}$ using $0.5 \mu \mathrm{M}$ of both rhodopsin and C3G. Samples were placed in the cuvette holder and the first spectrum was recorded at $20{ }^{\circ} \mathrm{C}$. Subsequently, the temperature was raised to $50{ }^{\circ} \mathrm{C}$ and the corresponding spectra were recorded every 5 minutes. All experiments with anthocyanins were carried out at three different $\mathrm{pH}$ values of 4,6 and 8. The data was analyzed by fitting the increase and decrease observed at $500 \mathrm{~nm}$ absorbance for regeneration studies and stability experiments respectively, to a non-linear regression function using Sigmaplot 10.0 scientific graphing software.

All thermal decay experiments of rhodopsin and metal ions were performed using a concentration of $0.5 \mu \mathrm{M}$ and $100 \mu \mathrm{M}$, respectively. The temperature was ramped at a rate of $0.5{ }^{\circ} \mathrm{C}$ per minutes for thermal melting experiments as needed. 


\subsubsection{Deglycosylation using PNGase-F}

For cleavage of oligosaccharide chains from rhodopsin glycosylation sites, purified WT and mutant rhodopsins were deglycosylated with PNGase F (0.5 units per $\mu \mathrm{g}$ of rhodopsin) from New England Biolabs. The purified proteins were first incubated with denaturing buffer $(0.5 \mathrm{M}$ sodium phosphate $\mathrm{pH}$ 7.5, 0.5\% SDS, $40 \mathrm{mM}$ DTT) for 1 hour, PNGase F and 1\% NP-40 were added and the reaction mixture further incubated for 6 hours at room temperature. Deglycosylated rhodopsin was analyzed by SDS PAGE as described in Section 2.4.8.

\subsubsection{Retinal Layer Thickness}

The protocol used for Outer Nuclear Layer (ONL) measurements was based on previous protocols (Faktorovich et al., 1990; Lewin et al., 1998). Samples of treated and untreated animals at 60, 90 and 150 days of age were used for the measurements. Mean ONL thickness was obtained from a single section of each animal that was nuclear stained with either Dapi or Ethidium bromide dyes, 1:1000 dilution as described in Section 2.2.6. As shown in Figure 2.4, both superior and inferior hemispheres were measured (measurements were done on both sides of the nerve). A total of 36 to 48 measurements of the ONL were made at 12-16 continuous fields around the entire retinal section ( 3 measurements per field). The total measurements were either averaged to provide a single value for each retina to allow for comparison between treated and non-treated groups, or plotted as a distribution of thickness across the retina. To start the

measurements, first the optical nerve was localized and the first set of measurements was obtained just after the head of the nerve (nerve head is characterized by an opening point where there is no photoreceptor). All the other measurements were taken more peripherally continuously.

Images were taken as described in Section 2.3.6. 
Figure 2.4 - Structure of the eye, showing how the retinal thickness measurements were performed.

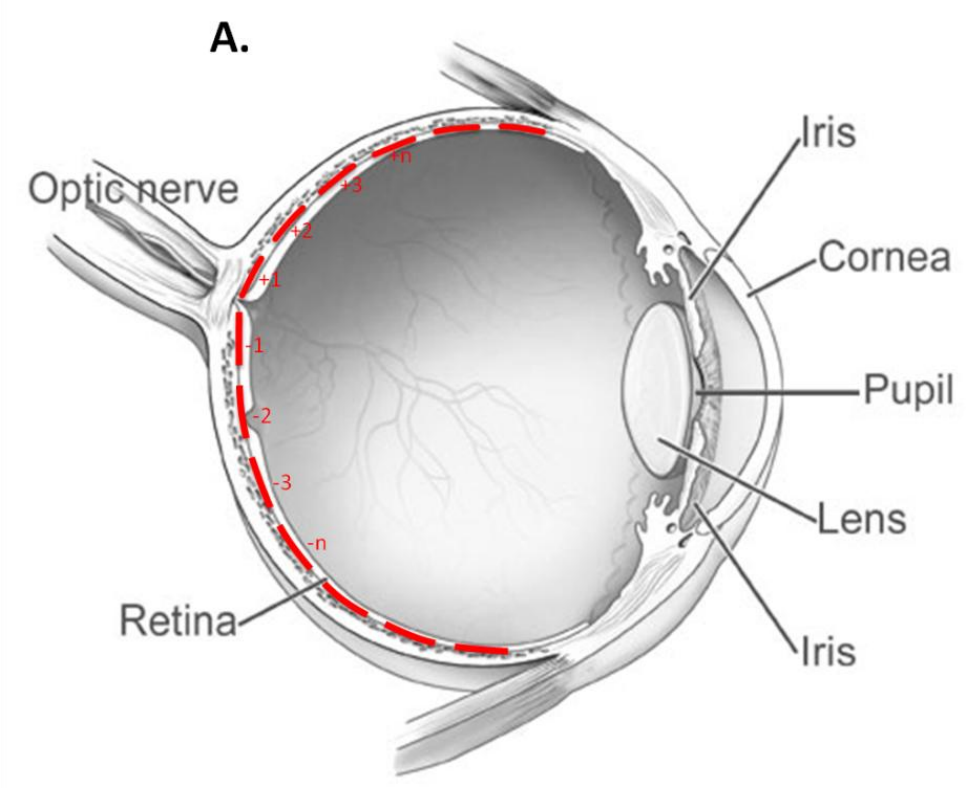

B.

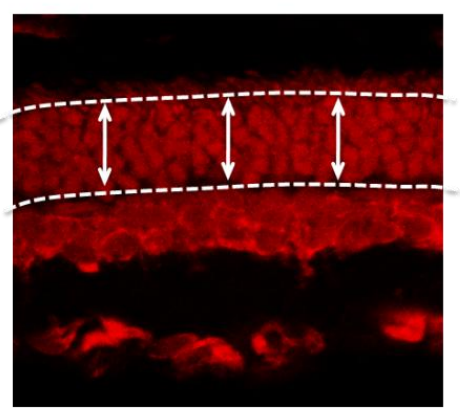

At first, the optical nerve was localized and the first picture was obtained just after the head of the nerve as shown in the figure, from there all other pictures were taken successively (A). Measurements were later done in three areas of each picture, and averaged in order to get one value for each area of the eye (B).

SOURCE: Eye diagram (A) is adapted from de National Eye Institute, National Institutes of Health. With permission. (B) Balen, 2012.

\subsubsection{TUNEL assay}

The TUNEL assay was used for detection of apoptotic cells (In Situ Cell Death Detection Kit, Roche Applied Science). Retinal sections were washed 3 times for 5 minutes in $0.05 \mathrm{M}$ phosphate buffer ( $\mathrm{pH} 7.4$ ), and incubated with $0.1 \%$ Na-citrate, $0.1 \%$ Triton-X 100 in $0.05 \mathrm{M}$ phosphate buffer ( $\mathrm{pH}$ 7.4) for 2 minutes in ice tray. After 3 washes with $0.05 \mathrm{M}$ phosphate buffer (pH7.4) for 5 minutes, the method for detecting DNA fragmentation by labeling the terminal end 
of the nucleic acids using the TUNEL kit was used. This consisted of incubation, for 1 hour at $37^{\circ} \mathrm{C}$, with the terminal deoxynucleotidyl transferase enzyme (TdT) and fluoroscein. DNA is reactive to fluorescein. Therefore, this procedure labels apoptotic cells. Reaction was stopped by washing with a solution of $4 \mathrm{mM}$ Tris- $\mathrm{HCl} \mathrm{pH} 8$ and $5 \mathrm{mM}$ EDTA for 5 minutes. Sections were again washed with $0.05 \mathrm{M}$ phosphate buffer $\mathrm{pH} 7.4$ for 5 minutes each, mounted with Vecta Shield (Vector Labs, Burlingame, CA) and analyzed using a fluorescence microscope.

\subsubsection{SDS PAGE Analysis}

SDS-Polyacrylamide gel electrophoresis (PAGE) was carried out according to (Laemmli, 1970). Resolving gels (10\% acrylamide) were poured after mixing $4 \mathrm{ml}$ of $30 \%$ acrylamide, $3 \mathrm{ml}$ of $1.5 \mathrm{M}$ Tris- $\mathrm{HCl} \mathrm{pH} 8.8,4.8 \mathrm{ml}$ of water, $120 \mu \mathrm{l}$ SDS (10\%), $80 \mu \mathrm{l}$ APS (10\%) and $6 \mu 1$ TEMED. The gels were poured in glass panes and overlaid with ultra-pure water to keep avoid contact with air and to allow the top of the gel to polymerize in a straight line. The gels were allowed to settle for $45-60$ minutes. Complete polymerization was evident by the appearance of a sharp line due to the refractive index change between the gel and the overlay. The overlay was replaced with stacking gel with the comb in place. Stacking gels (5\% acrylamide) were poured after mixing $680 \mu \mathrm{l}$ of $30 \%$ acrylamide, $1 \mathrm{ml} 0.5 \mathrm{M}$ Tris- $\mathrm{HCl} \mathrm{pH} \mathrm{6.8,2.2} \mathrm{ml} \mathrm{water,} 40 \mu \mathrm{SDS}$ (10\%), $80 \mu \mathrm{l}$ APS (10\%) and $4 \mu \mathrm{l}$ TEMED. Laemmli protein loading dye (Sigma, S-3401) was used in 1:1 ratio to load proteins on the gel. Gels were run at $200 \mathrm{~V}$ till the dye front reached the bottom of the gel.

Coomassie staining was done by heating the polyacrylamide gel with $45 \%$ methanol, $9 \%$ acetic acid, $0.2 \%$ Coomassie blue buffer for three times (10 minutes each) in a microwave. The gel was then de-stained with $25 \%$ methanol, $10 \%$ acetic acid. Silver staining was done following the instruction sheet in SilverSNAP Stain kit (Thermo Scientific, Waltham, MA). 


\subsubsection{Western Blot}

The protein samples were mixed with SDS-PAGE buffer and were separated by electrophoresis. Normally it was run for $200 \mathrm{~V}$ for 1-1.5 hours or until all the visual bands are on the bottom of the cassette, on a 10\% SDS-PAGE gel (running buffer was from BIO-RAD: $100 \mathrm{mM}$ Tris, $100 \mathrm{mM}$ Tricine and $0.1 \%$ SDS). Proteins were transferred from the gels to polyvinylidene difluoride membranes (PVDF, BIO-RAD). The PVDF membrane was activated by soaking in methanol. The cassette was assembled in a container with transfer buffer $(5 \mathrm{mM}$ Tris, $192 \mathrm{mM}$ glycine, and 20\% methanol) by making a sandwich with sponge/tissue paper/gel/membrane/tissue/sponge with the membrane facing the anode side of the cassette. This was then placed in the blotting container and the blot was run at $100 \mathrm{~V}$ for 1 hour. The membranes were then processed immediately to detect proteins.

To process the membranes, a common procedure for detecting proteins on the membranes that are transferred from gels or for membranes from dot blots was used. The membranes were blocked in blocking buffer: PBS buffer with $0.05 \%$ Tween 20 and 5\% dry milk powder at room temperature for $30-60$ minutes or at $4{ }^{\circ} \mathrm{C}$ if left over night. The blots were then incubated for 1 hour at room temperature in 1D4 primary antibody diluted to 1:25000 in PBS buffer with 0.05\% Tween 20. The blots were washed three times wherein each wash was for 15 minutes in wash buffer (PBS buffer with 0.05\% Tween 20). Later, the blots were incubated in Horseradish

peroxidase conjugated goat anti-mouse secondary antibodies (BIO-RAD) diluted 1:50000 in wash buffer for 30 minutes at room temperature. The blots were washed thrice for 15 minutes in wash buffer. The blot was then incubated with $6 \mathrm{ml}$ of West Pico Chemiluminescent Substrate from Pierce, then exposed to film in the dark room and developed.

\subsubsection{Dot Blot}

Dot blots were used to evaluate relative levels of protein expression in cells or membranes. Dilutions of the samples were prepared in a 96 well plate as desired in PBS buffer. Pre-wet $0.2 \mu \mathrm{m}$ nitrocellulose membrane in PBS buffer. (Note: PVDF membranes are not recommended). The dot blot apparatus was set up with the blot. Using a multichannel pipette, $200 \mu \mathrm{l}$ of PBS buffer was added and the blot was washed once. 10 - $20 \mu \mathrm{l}$ of sample was 
transferred on to the blot using the multichannel pipette. The sample was left on the blot for at least 5 minutes. After this, just enough vacuum was applied to drain the samples. The wells were washed two times with $200 \mu$ of PBS buffer. Minimal vacuum was applied to drain the buffer. The blot was then removed and the membrane was developed as a regular Western blot. 


\section{CHAPTER 3: MOLECULAR CHARACTERIZATON OF WT, N15S AND P23H MUTANTS OF RHODOPSIN IN MAMMALIAN CELLS}

\subsection{SUMMARY}

To date, more than 150 mutations associated with RP are known in rhodopsin. Most of these mutations cause misfolding of rhodopsin in vitro, and it has been hypothesized that molecular instability of the rhodopsin structure is responsible for disease severity in patients. Here, two RP mutations, N15S and P23H, located in the extracellular domain of rhodopsin were studied to test this hypothesis. Both mutations have been shown to misfold in cells (Kaushal and Khorana, 1994; Tam and Moritz, 2009). In this study, a quantitative comparison of molecular properties was done using thermal denaturation, total reflectance Fourier Transform Infrared (ATR/FT-IR) and fluorescence spectroscopy, SDS-PAGE mobility, glycosylation studies, and subcellular localization using confocal microscopy. The study reveals that the two mutations differ in characteristics and severity of defects, despite the global classification into the same classes. N15S is only slightly defective in structure, stability and cellular localization, as seen by its expression levels, stability studies at $37{ }^{\circ} \mathrm{C}$ and $55{ }^{\circ} \mathrm{C}$, glycosylation pattern and in cell localization. $\mathrm{P} 23 \mathrm{H}$, on the other hand, is severely impaired at both molecular and cellular levels. Its expression level was less than WT rhodopsin and stability studies at $37{ }^{\circ} \mathrm{C}$ and $55{ }^{\circ} \mathrm{C}$ indicate an approximately 4-fold decrease in stability, measured by the loss of $500 \mathrm{~nm}$ chromophore rate. Characterization using FTIR also showed that N15S is less misfolded and more WT-like as compared to $\mathrm{P} 23 \mathrm{H}$. $\mathrm{P} 23 \mathrm{H}$ presented an increased percentage of random coil fractions at the cost of $\alpha$-helix, $\beta$-sheet and turn folded fractions.

\subsection{INTRODUCTION}

Rhodopsin as the first identified genetic cause of the RP disease is a primary potential target for treating RP. Mutations in the rhodopsin gene $(R H O)$ are the primary cause for autosomal dominant RP (ADRP). Clinical and functional in vitro and in vivo studies have led to a classification of RHO mutants (Cideciyan et al., 1998; Kaushal and Khorana, 1994; Stojanovic and Hwa, 2002; Sung et al., 1991, 1993): 
(a) Clinical classification divides ADRP into two classes - classes A and B (Cideciyan et al., 1998). Class A is characterized by severe rod function abnormalities based on electroretinogram (ERG) and perimetry measurements. This class consistently results in early night blindness, while there is measurable cone-mediated activity even later in life. On the other hand, Class B shows measurable rod function (partial ERG preservation) and well preserved cone function. It is characterized by variable onset of night blindness.

(b) In vitro classification of $\mathrm{ADRP}$ mutants has been carried out based on their molecular phenotype. Three classes (I-III) have been proposed (Kaushal and Khorana, 1994). Class I resembles WT rhodopsin where the mutants fold correctly, bind the 11-cis retinal chromophore normally, but are inefficient in activation of transducin. Class II mutants do not traffic out of the endoplasmic reticulum, and do not bind 11-cis retinal. Class III mutants are expressed at low levels, poorly form rhodopsin chromophore, and are also retained in the endoplasmic reticulum, presenting high mannose glycosylation.

Bridging the gap between the classifications of mutants based on disease phenotype in patients and the classifications based on in vitro biochemical properties of RHO mutations requires a detailed comparison between the clinical manifestations of the mutations and the molecular properties of the proteins. Towards this goal, several class B ADRP patients, carrying mutations in different domains of rhodopsin, namely in the extracellular domain, in the second intradiscal loop (P180A and G188R), and in the cytoplasmic domain, at the end of helix III (R135L and R135W) were previously studied (Iannaccone et al., 2006). The degrees of severity and progression rates in patients correlated with the computationally predicted and experimentally confirmed molecular properties of the respective rhodopsin protein (Iannaccone et al., 2006). Here these studies are extended to two additional mutations in the extracellular domain, namely N15S and P23H mutations at the N-terminus of rhodopsin (Figures 4.1 and 4.2). 
Figure 3.1 - Secondary structure model of rhodopsin.

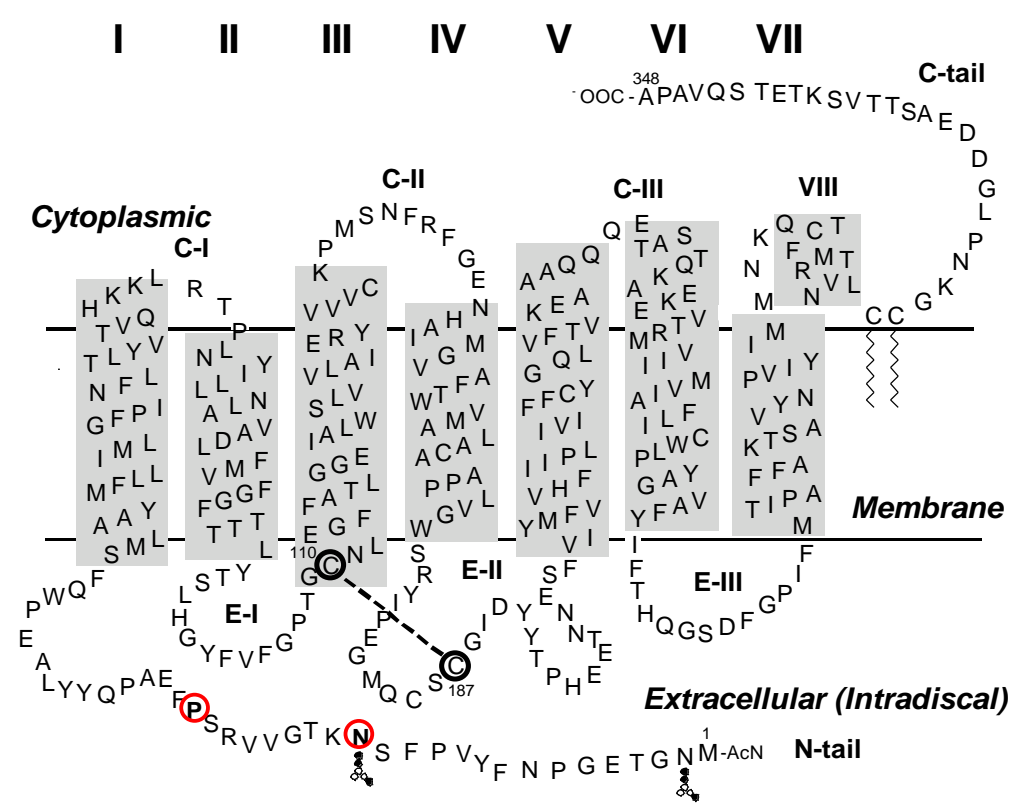

The two mutations investigated in this chapter, $\mathrm{N} 15 \mathrm{~S}$ and $\mathrm{P} 23 \mathrm{H}$, are showed in red.

SOURCE: Balen, 2012

Figure 3.2 - Tertiary structure of rhodopsin $(1 \mathrm{L9H})$.

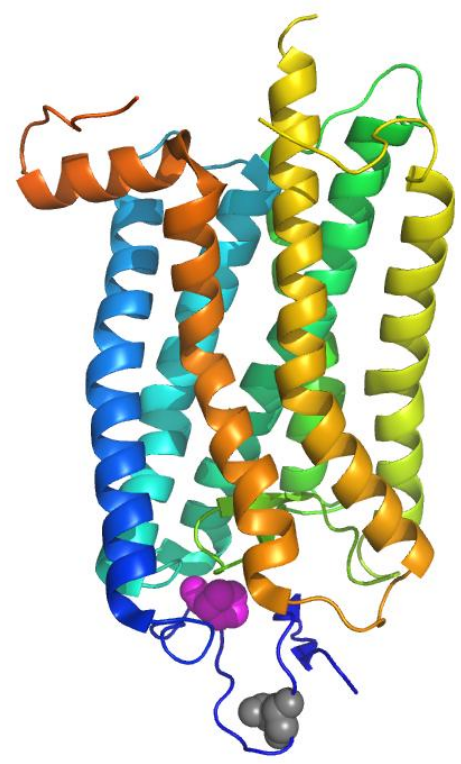

N15 (grey) and P23 (magenta) residues are depicted in the bottom of the figure.

SOURCE: Balen, 2012 
P23H was first identified as an ADRP mutation by Dryja and co-workers (Dryja et al., 1990). It was historically the first single change in the human rhodopsin gene associated with ADRP. $\mathrm{P} 23 \mathrm{H}$ is one of the most frequent mutations observed in ADRP patients. Its clinical phenotype is class B (Cideciyan et al., 1998) and its molecular phenotype class III based on the observation that it yields much less chromophore with 11-cis retinal than WT. It also displays aberrant trafficking to the cell surface and its bleaching behavior is abnormal (Kaushal and Khorana, 1994). Severe impairment of folding and 11-cis retinal binding was confirmed by a number of studies (Krebs et al., 2010; Liu et al., 1996; Tam and Moritz, 2009).

N15 is one of the two glycosylation sites of rhodopsin (Hargrave, 1977). In addition to the previously reported mutations, $\mathrm{T} 4 \mathrm{~K}$ and $\mathrm{T} 17 \mathrm{M}$, which are located in proximity of the glycosylated sites, N15S is the third rhodopsin mutation causing ADRP by affecting an essential residue at one of the two glycosylation sites (Kranich et al., 1993). The non-glycosylated WT is able to bind 11-cis retinal, but is reduced in its ability to activate transducin. Furthermore, mutations at this site have shown poor folding and the mutant proteins were defective in transport to the cell surface (Kaushal et al., 1994). In addition, although the mutant with N15 substitution is able to bind 11-cis retinal, the extent of retinal binding is lower than WT; the ratio A280/A500 was 6.2 instead 1.8 of that of WT rhodopsin deglycosylated (Kaushal et al., 1994), indicating a folding impairment. Even though this mutation was not studied in the original classification of ADRP mutations (Kaushal and Khorana, 1994), its molecular phenotype fits the description of class III ADRP mutations.

Thus, prior studies established that both mutations, N15S and $\mathrm{P} 23 \mathrm{H}$, located at about the same region of rhodopsin, namely the N-terminus in the extracellular domain, are grouped in the same clinical phenotype class and molecular properties class (Figures 3.1 and 3.2). This provides us with an opportunity to test the hypothesis that clinical phenotype and molecular properties are related at a finer grained level than the previous study (Iannaccone et al., 2006). To this end, a molecular characterization was performed here. These studies included subcellular localization using confocal microscopy, SDS-PAGE mobility, glycosylation studies, thermal stability and secondary structure content using ATR/FT-IR. It was found that despite the global classification into the same classes both from a clinical and a molecular perspective, a quantitative comparison of molecular properties reveals that the two mutations differ in molecular characteristics and phenotype severity. 


\subsubsection{Expression and purification of mutant and wt rhodopsin}

\subsubsection{Transient transfection of WT, N15S and P23H mutants of rhodopsin}

The N15S and $\mathrm{P} 23 \mathrm{H}$ mutations were expressed using transient transfection of COS-1 cells, as described in Section 2. Figure 3.3 shows the UV/Vis absorption spectrum of rhodopsin WT and mutants, which after purification were reconstituted with 11-cis retinal. The protein in each case was eluted with $0.05 \%$ (w/v) $\mathrm{DM}$ in $2 \mathrm{mM} \mathrm{Na} 2 \mathrm{HPO}_{4} / \mathrm{NaH}_{2} \mathrm{PO}_{4}(\mathrm{pH}$ 6.0) containing $70 \mu \mathrm{M}$ C-terminal nonapeptide or $0.05 \%(\mathrm{w} / \mathrm{v}) \mathrm{DM}$ in PBS as indicated. Since the rhodopsin mutants present both folded and unfolded portions, purification of the mutants was done first with PBS in order to quantify the total amount of protein (including folded and misfolded portion). The $\mathrm{A}_{280}: \mathrm{A}_{500}$ absorbance ratio and their standard deviations in PBS were 1.86 \pm 0.79 , $13.39 \pm 2.60$ and $5.67 \pm 0.3$ for $\mathrm{WT}, \mathrm{P} 23 \mathrm{H}$ and N15S, respectively.

Figure 3.3 - Purification profile of WT rhodopsin (Blue) and mutants P23H (Red) and N15S (Green).

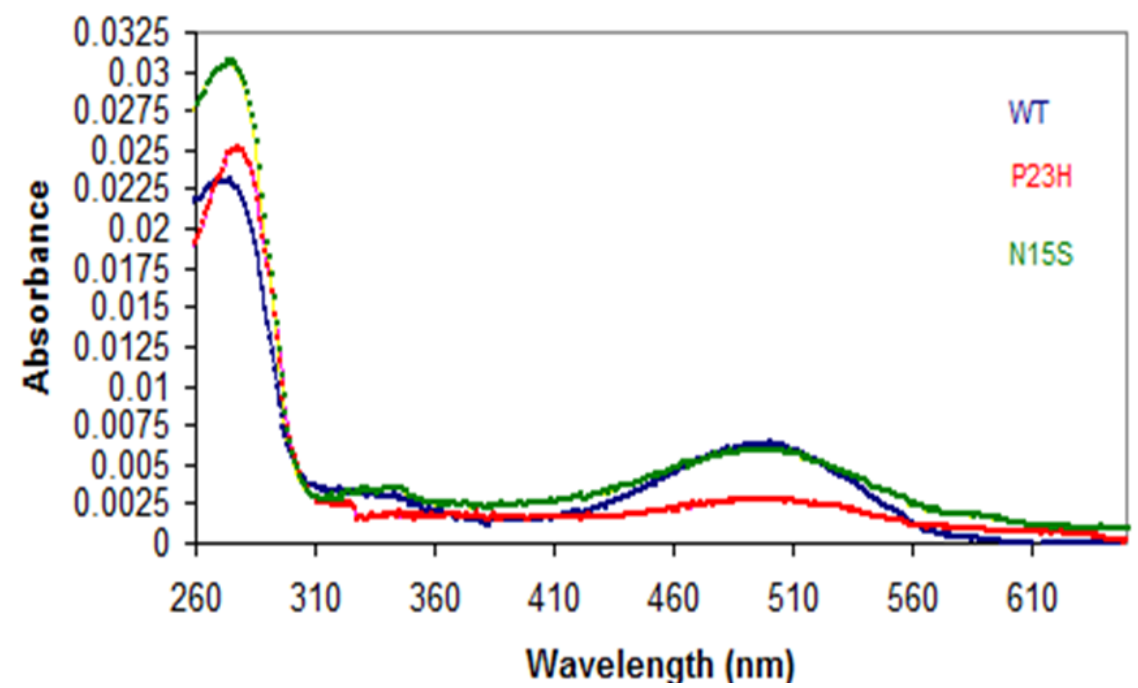

SOURCE: Balen, 2012

The stability of rhodopsin in $2 \mathrm{mM}$ sodium phosphate buffer (Napi) pH6, 0.05\% DM was estimated by measuring the loss of $500 \mathrm{~nm}$ chromophore absorbance over time (Figure 3.4). WT 
rhodopsin showed a $10 \%$ decrease over a period of 1 hour, while for $\mathrm{N} 15 \mathrm{~S}$ and $\mathrm{P} 23 \mathrm{H}$, which where purified with PBS as the buffer, the decrease was $20 \%$ and $80 \%$, respectively.

Figure 3.4 - Rhodopsin stability measured by loss of $500 \mathrm{~nm}$ chromophore over time at $55{ }^{\circ} \mathrm{C}$.

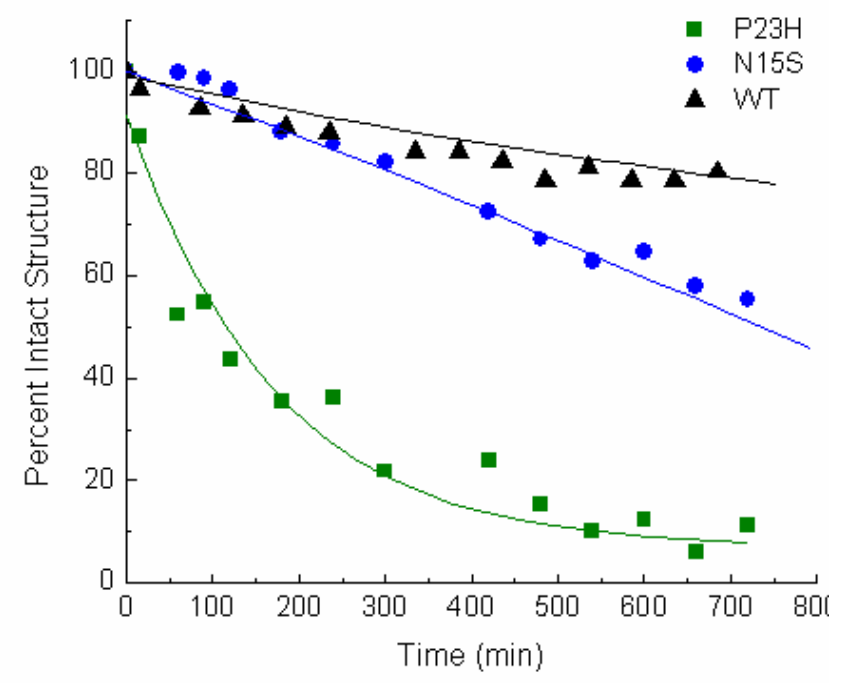

Absorbance spectra were measured in time intervals and the decrease in absorbance at $500 \mathrm{~nm}$ was expressed at percent of intact structure.

SOURCE: Balen, 2012

\subsubsection{Creation of stable cell lines of mutant N15S and P23H}

The yields of protein using transient transfections were low. To obtain larger amounts of purified and folded rhodopsin necessary to perform many of the biophysical techniques that would allow the characterization of the mutant proteins, stable cell lines of the two mutants were created using HEK293 cells (as described in Section 2).

\subsubsection{Reconstitution of mutant and WT rhodopsin with 9-cis or 11-cis retinal}

Initially, the mutant stable cell lines were treated and reconstituted with 9-cis or 11-cis retinal after expression immediately prior to purification as it was performed for transient transfected COS-1 cells. The yield of folded purified rhodopsin improved. However, the amounts were still not sufficient for most biophysical studies. Therefore, another protocol was tested (for 
details see Section 2), where reconstitution of rhodopsin was done during the synthesis of the protein. The addition of retinal at this earlier point had proven to be efficient to help the correct folding of many rhodopsin mutations, including P23H (Krebs et al., 2010).

\subsubsection{Addition of 9-cis or 11-cis retinal to cells during synthesis}

Following the protocol used by Krebs and collaborators (Krebs et al., 2010), 9-cis or 11cis retinal were added directly to the cells growing on dishes during synthesis of the protein. This protocol made possible the purification of almost WT-like amounts of protein for both mutants.

After purification, the thermal stability of N15S and P23H mutants, in comparison to WT purified from HEK293 cells expressing rhodopsin in the presence of retinal, was investigated by following the loss of $500 \mathrm{~nm}$ absorbance as a function of time at $37{ }^{\circ} \mathrm{C}$. The result is shown in Figure 3.5. The decrease in absorbance is expressed as percent of initial absorbance (at $t=0)$. At

this temperature, there is no loss of chromophore for WT rhodopsin. The thermal stability of N15S was noticeably less as compared to that of WT, while P23H was highly unstable. The halflife and standard deviation of the $\mathrm{N} 15 \mathrm{~S}$ and $\mathrm{P} 23 \mathrm{H}$ were $101.0 \pm 27.6$ and $52.6 \pm 13.1 \mathrm{~min}$, respectively. Since WT rhodopsin is stable at this temperature and there is no significant decay, the half life was not calculated. Similar relative stability results were observed at higher temperature $\left(55^{\circ} \mathrm{C}\right)$, when rhodopsin transiently expressed and reconstituted from COS-1 cells at harvesting was investigated for comparison (Figure 3.4). These results demonstrate that despite the expression level and folding improved upon addition of retinal during expression, the purified proteins show the same stability pattern regardless the source. Both mutants are less stable than WT, with P23H being much less stable than N15S.

The increased amount of folded chromophore-containing mutant rhodopsin was quantified through purification and spectral analysis. Expression of the N15S and P23H mutants in HEK293 stable cell lines in the presence of 9-cis retinal during opsin expression instead of adding it at the point of reconstitution, as it is typically done, showed a large improvement in the yield of folded protein. This was judged by the difference in absorbance spectra of dark and illuminated detergent-solubilised membranes, as well as after purification using 1D4-sepharose. The yields of correctly folded protein were now typically WT-like (data not shown). This confirms that both 9-cis and 11-cis can work as pharmacological rescue agents for correctly 
folding the protein (Krebs et al., 2010). The previous observation was extended here to the case of N15S.

Figure 3.5 - Rhodopsin stability measured by loss of $500 \mathrm{~nm}$ chromophore over time at $37^{\circ} \mathrm{C}$.

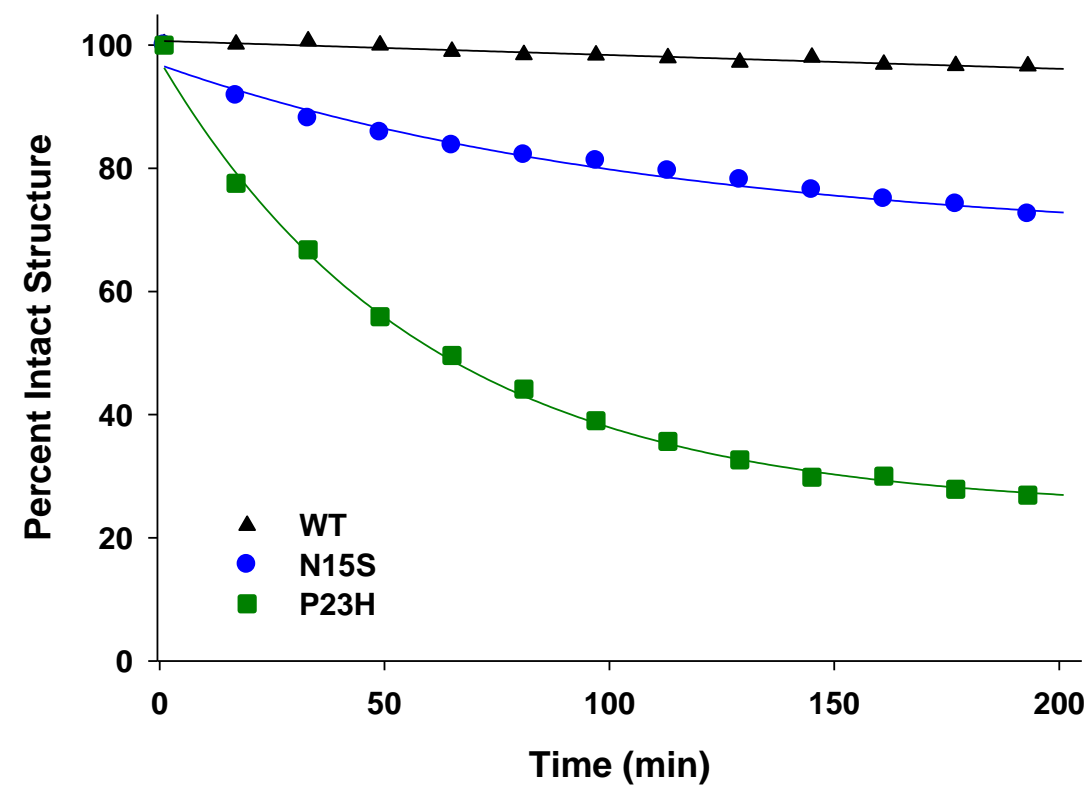

Absorbance spectra of rhodopsin, WT, and rhodopsin mutants, N15S and P23H expressed in the presence of 9-cis retinal at $37{ }^{\circ} \mathrm{C}$ were measured over time, and the decrease in absorbance at $500 \mathrm{~nm}$ was expressed at percent of intact structure.

SOURCE: Balen, 2012

\subsubsection{Molecular characterization of WT, N15S and PS23H mutants rhodopsin}

\subsubsection{Glycosylation and aggregation of WT, N15S and P23H in COS-1 cells}

As described above, the amino terminus of rhodopsin carries two glycosylation sites, N2 and N15 (Hargrave, 1977). For cleavage of oligosaccharide chains from rhodopsin glycosylation sites, treatment with the enzyme PNGase F (Peptide: N-Glycosidase F) was performed. This enzyme is a native glycoaminidase that cleaves the link between asparagine and $\mathrm{N}$ acetylglucosamines, allowing the study of $\mathrm{N}$-linked carbohydrates in a single protein. Previous studies with rhodopsin have shown the difference in apparent molecular weight of rhodopsin and different mutants on SDS-PAGE and Western blot (Iannaccone et al., 2006). Glycosylation was 
assessed before (-) and after (+) treatment with PNGase F. The results are shown in Figure 3.6. WT rhodopsin displays the expected highly diffuse bands corresponding to monomeric and dimeric rhodopsin before PNGase F (-) treatment due to heterogeneous glycosylation. Distinct bands were observed after the treatment with PNGase F (+), as expected.

Figure 3.6 - Western-blot of WT and rhodopsin mutants (N15S and P23H) expressed transiently in COS-1 cells.

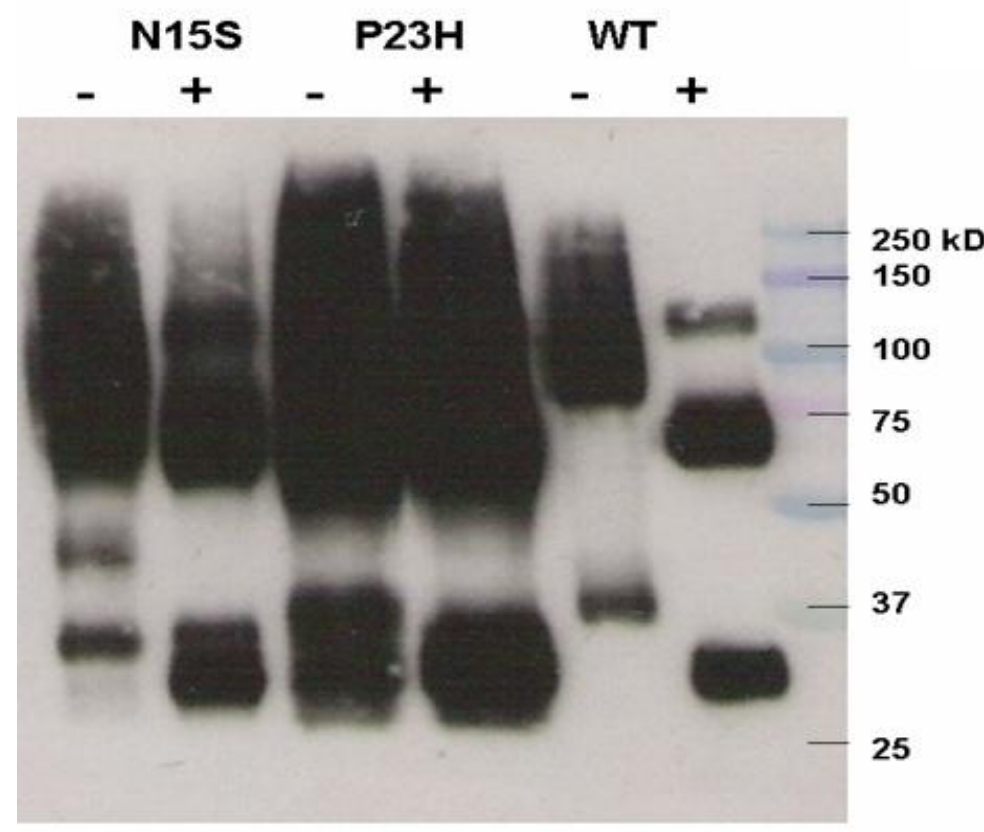

Protein concentrations were adjusted according to expression level and approximately $1 \mu \mathrm{g}$ was loaded. In each case, equivalent amounts were analyzed before (-) and after (+) treatment with PNGaseF.

SOURCE: Balen, 2012

In the case of N15S, already in the absence of PNGase F, there was evidence for unglycosylated species at about $35 \mathrm{kD}$ as well as a distinct band at about $45 \mathrm{kD}$, consistent with the retention of some protein in the ER and subsequent trafficking differently from WT. The bands are more distinct than the WT because one glycosylation site is absent in this mutant, reducing the complexity of heterogeneous glycosylation in this mutant. The intensity of aggregated bands was significantly higher in N15S than in WT. After PNGase F treatment, the protein appeared essentially WT-like, demonstrating the relatively mild phenotype of this mutant.

In the case of $\mathrm{P} 23 \mathrm{H}$, the proportion of unglycosylated species was much higher than in N15S, with glycosylated and non-glycosylated monomeric species being similar in intensity. 
Aggregation was higher in $\mathrm{P} 23 \mathrm{H}$ than in N15S. Thus, the degree of aggregation and aberrant glycosylation in $\mathrm{P} 23 \mathrm{H}$ was more severe than that of N15S.

\subsubsection{Intracellular localization studies using confocal microscopy}

To directly compare subcellular localization of $\mathrm{N} 15 \mathrm{~S}$ and $\mathrm{P} 23 \mathrm{H}$ mutants, immunofluorescence of these mutants was carried out in transient transfections of COS-1 cells and in stable HEK293 cells inducibly expressing WT, N15S and P23H mutants of rhodopsin. The results are shown in Figure 3.7. The cells transfected with WT rhodopsin show high levels of receptor expression, which is distributed all over the cells in both transiently and stably expressing cells (Figure 3.7A). Rhodopsin is trafficked to the plasma membrane as evident from the characteristic orange color which is a result of the co-localization of the actin (red) and rhodopsin (green) at the cell boundaries. The localization of $\mathrm{P} 23 \mathrm{H}$ is restricted to the cytoplasm (Figure 3.7C) confirming the previously suspected misfolding and trafficking problems of this mutant (Saliba et al., 2002). The majority of $\mathrm{P} 23 \mathrm{H}$ is localized adjacent to the nucleus as aggresomes, in agreement with previous observations (Saliba et al., 2002). The N15S mutant also shows aggregation as seen in the $\mathrm{P} 23 \mathrm{H}$ mutant but with some fraction trafficked onto the membrane (Figures 3.7B and 3.7D). These results are in agreement with previous studies of a N15D mutation (Kaushal et al., 1994). 
Figure 3.7 - Localization of WT and mutant rhodopsin.

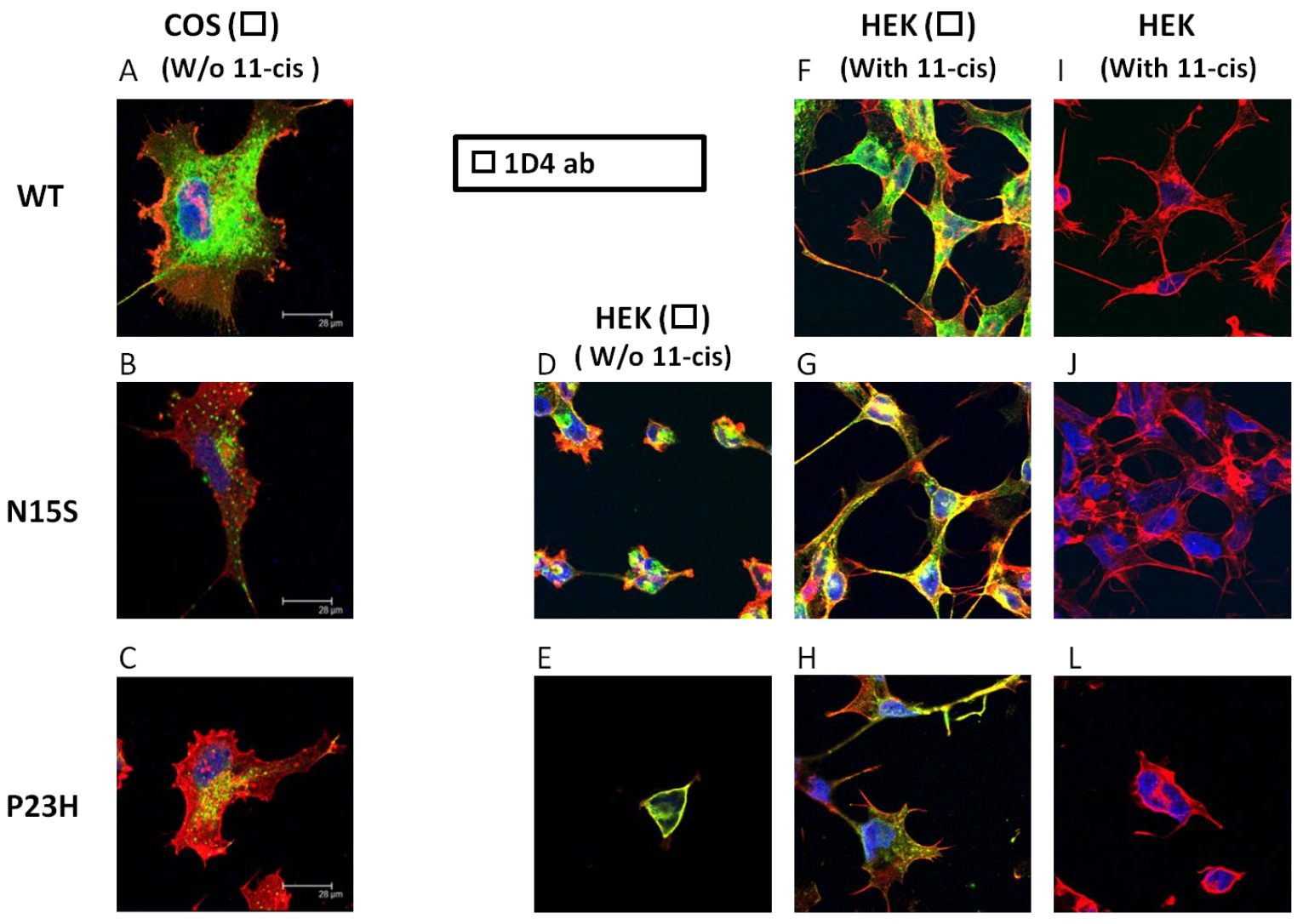

COS-1 cells transfected with WT, P23H and N15S rhodopsin mutants (A-C) and HEK293-stable cells WT, P23H and N15S mutants (D-L). Open square: 1D4 ab. Cells are stained with anti-rhodopsin C-terminal antibody 1D4 (Green), actin marker - Rhodamine-Phalloidin (Red) and nuclear stain - DRAQ-5 (Blue). The actin and nuclear markers help delineate the individual cells and their boundaries. In COS-1 cells, WT rhodopsin is observed only on the surface while $\mathrm{P} 23 \mathrm{H}$ was only observed in aggresomes and $\mathrm{N} 15 \mathrm{~S}$ was present both on the surface and as aggresomes. For HEK293 cells, WT rhodopsin and the mutants P23H and N15S were observed to be at the surface of the cells. Aggresomes were also observed in the mutants.

SOURCE: Balen, 2012

\subsubsection{Rescue of chromophore in N15S and P23H mutants by the addition of retinal}

Since it was previously shown that misfolded $\mathrm{P} 23 \mathrm{H}$ rhodopsin can be pharmacologically rescued by the addition of 11-cis or 9-cis retinal to cell cultures (Krebs et al., 2010), the subcellular localization of the mutants after treatment with 11-cis retinal was investigated. In contrast to the abnormal cell morphology and receptor distribution of N15S and P23H mutants in HEK293 cells without the addition of 11-cis retinal, on addition of 11-cis retinal, N15S and $\mathrm{P} 23 \mathrm{H}$ cells showed WT-like receptor expression and distribution. These results suggest that N15S and $\mathrm{P} 23 \mathrm{H}$ mutants are rescued by the addition of 11-cis retinal. However, the rescue of N15S appears 
to be better than $\mathrm{P} 23 \mathrm{H}$, as evidenced by the overall uniform distribution in the cell (Figure 3.7F and 3.7G). As controls, background fluorescence of WT, N15S and P23H treated with 11-cis retinal were immunostained without the primary (1D4) antibody, shown in Figures 3.7I-3.7L. Furthermore, uninduced control cells treated with 11-cis retinal showed normal cell morphology and cell distribution (data not shown).

\subsubsection{Structural characterization using FTIR spectroscopy analysis of WT, N15S and P23H mutants}

The above analyses demonstrate that the two mutants differ in folding, retinal-protein interactions and stability. To further corroborate these findings, a detailed analysis of protein secondary structure using FTIR spectroscopy was carried out. Deconvoluted ATR-FTIR spectra in the amide I region $\left(1600-1700 \mathrm{~cm}^{-1}\right)$ with center at around $1650 \mathrm{~cm}^{-1}$, corresponding mainly to $\mathrm{C}=\mathrm{O}$ stretching of the peptide backbone, are shown in Figure 3.8. The percentages of the secondary structures, estimated by integrating the intensities for each secondary structure element and expressing them as a fraction of the total amide I region, are listed in Table 3.1. Overall, the amide I bands of N15S and P23H appear broader as compared to WT rhodopsin (Figure 3.8). In WT, the content of $\alpha$-helix, $\beta$-sheet, random coil and turns in aqueous conditions were $63 \%, 16 \%$, $12 \%$ and $9 \%$, respectively. The observed estimates are in good agreement with the estimated secondary structure content from the X-ray crystal structure of rhodopsin. Using PDB ID 1L9H, $64 \%, 3 \%, 12 \%$ and $22 \%$ are calculated for $\alpha$-helix, $\beta$-sheet, random coil and turns, respectively. In the case of the N15S mutant, a decrease in $\alpha$-helix content (42\%), and an increase in $\beta$-sheet (25\%), random coil $(21 \%)$ and turns (12\%) was observed when compared to WT. The P23H mutant exhibited even more severe structural defects when compared to WT: a strong increase in the random coil fraction (52\%) were observed, and the $\alpha$-helix, $\beta$-sheet, and turn fractions decreased to $32 \%, 10 \%$, and $6 \%$, respectively. These results indicate that the structure of N15S is more folded as compared to $\mathrm{P} 23 \mathrm{H}$, based on the increase in helical fraction and a decrease in random coil content.

Next, the reasons why the N15S mutant showed an increase in $\beta$-sheet and turn contents as compared to both, WT and the $\mathrm{P} 23 \mathrm{H}$ mutant, was investigated. To overcome the limitation that the bands corresponding to $\alpha$-helix, random coil and $\beta$-sheet severely overlap and are thus 
difficult to separate under aqueous condition, ATR-FTIR spectra of the samples were recorded in $\mathrm{D}_{2} \mathrm{O}$. Backbone $\mathrm{H} / \mathrm{D}$ exchange leads to shifts in the bands corresponding to secondary structure elements in the amide I region, with bands originating from helices shifting less than random coil bands resulting in a skewed amide I band (Figures 3.8 D-F). The re-estimated secondary structure analysis of WT rhodopsin showed a decrease in $\alpha$-helix (59\%) and $\beta$-sheet (12\%) along with an increase in random coil (19\%) and turns (10\%). An increase in $\alpha$-helix (48\%) and turns (19\%) and a decrease in $\beta$-sheet $(18 \%)$ and random coil (15\%) fraction were observed for N15S and for $\mathrm{P} 23 \mathrm{H}$ an increase in $\beta$-sheet $(30 \%)$ and turns (13\%), a decrease in random coil (24\%) and a similar fraction of $\alpha$-helical fraction (33\%) were observed. These results indicate that structural features of the N15S mutant were more similar to WT structural features than those of the P23H mutant. The overall increase in $\beta$-sheet and random coil fractions and a decrease in $\alpha$-helix content clearly suggest that the $\mathrm{P} 23 \mathrm{H}$ mutant is less folded as compared to N15S and WT. Further, an increase of $18 \%$ in $\beta$-sheet content compared to WT (30\% vs $12 \%$ ) also indicates that this could be due to aggregation of the $\mathrm{P} 23 \mathrm{H}$ mutant. These results support the conclusion that the N15S mutant is less misfolded and more WT-like as compared to the P23H mutant. 
Figure 3.8 - The ATR / FTIR amide I band region along with the de-convoluted spectra corresponding to the different secondary structure fractions of (A) WT, (B) N15S and (C) P23H mutants of rhodopsin in $\mathrm{H}_{2} \mathrm{O}$ and (D) WT, (E) N15S and (F) P23H mutants of rhodopsin in $\mathrm{D}_{2} \mathrm{O}$.

A.

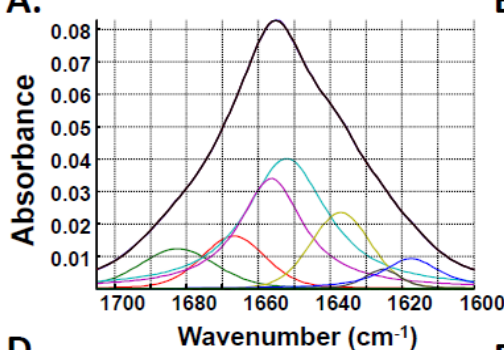

D.

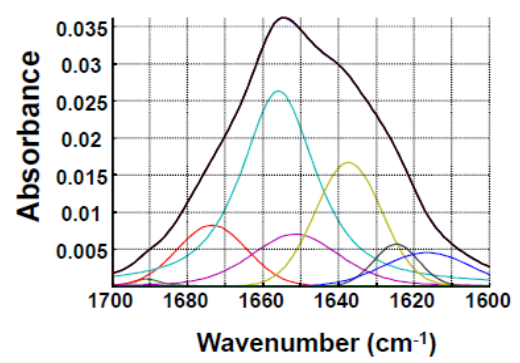

B.

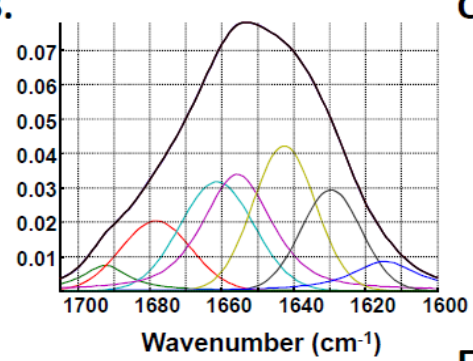

E.

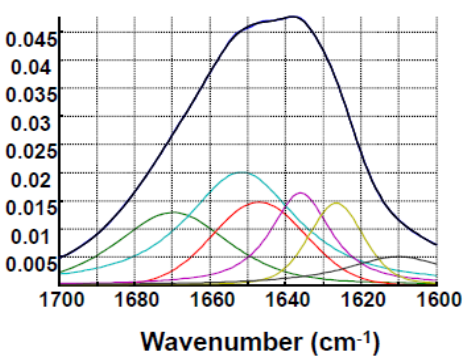

C.

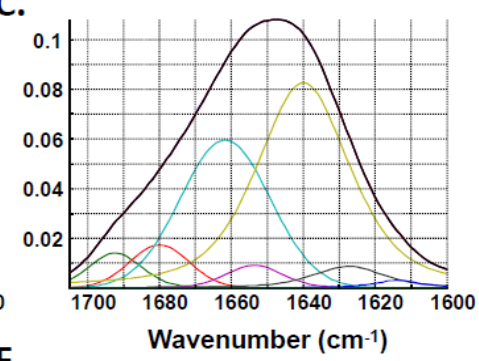

F.

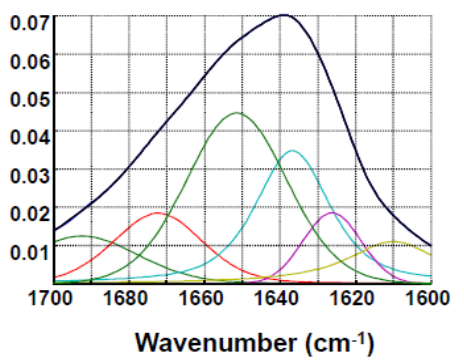

The bands between AA-BB cm- 1 were assigned to $\beta$-sheets, CC-DD cm- 1 to turns, EE-FF cm- 1 to $\alpha$-helices, and GG-HH cm-1 to random coil.

SOURCE: Balen, 2012

Table 3.1 - The table shows the calculated secondary structure content of the WT and the mutants N15S and P23H before and after H/D exchange from the ATR-FTIR spectra recorded in the amide I region.

\begin{tabular}{ccccccccc}
\hline $\begin{array}{c}\text { Secondary Structure Details } \\
\boldsymbol{\%}\end{array}$ & \multicolumn{2}{c}{ WT_PBS } & N15S_PBS & P23H_PBS & \multicolumn{2}{c}{ P23H_NaPi } \\
\hline & $\mathbf{H}_{\mathbf{2}} \mathbf{0}$ & $\mathbf{D}_{\mathbf{2}} \mathbf{0}$ & $\mathbf{H}_{\mathbf{2}} \mathbf{0}$ & $\mathbf{D}_{\mathbf{2}} \mathbf{O}$ & $\mathbf{H}_{\mathbf{2}} \mathbf{0}$ & $\mathbf{D}_{\mathbf{2}} \mathbf{O}$ & $\mathbf{H}_{\mathbf{2}} \mathbf{0}$ & $\mathbf{D}_{\mathbf{2}} \mathbf{O}$ \\
Helix & 63 & 59 & 42 & 48 & 32 & 33 & 62 & 48 \\
Beta sheet & 16 & 12 & 25 & 18 & 10 & 30 & 25 & 27 \\
Random coil & 12 & 19 & 21 & 15 & 52 & 24 & 8 & 18 \\
Turns & 9 & 10 & 12 & 19 & 6 & 13 & 5 & 8 \\
\hline
\end{tabular}

Increased beta sheet and decreased helix content was observed for the P23H mutant, while N15S was WT-like.

SOURCE: Balen, 2012 


\subsubsection{Comparison of Meta II decay rates}

As the stability of $\mathrm{P} 23 \mathrm{H}$ and $\mathrm{N} 15 \mathrm{~S}$ was less comparable to WT rhodopsin both from COS-1 and HEK293 cells, the difference in stability was further assessed after light activation. The decay of Meta II to free retinal and opsin (Meta II decay) at $20{ }^{\circ} \mathrm{C}$ was quantified using fluorescence spectroscopy (Farrens and Khorana, 1995). The increase in fluorescence upon lightactivation was fitted to a non-linear regression function in order to calculate the Meta II half-life $\left(t_{1 / 2}\right)$ for WT, N15S and P23H (Figure 3.9). The estimated $t_{1 / 2}$ values and standard deviation for a set of 2 experiments (WT-retina, plotted here for comparison), 3 experiments (WT and mutants) were $13.37 \pm 0.47,15.2 \pm 0.99,31.33 \pm 5.32$ and $45.00 \pm 15.72$ for WT-retina, WT, P23H and N15S, respectively. The fluorescence intensity at saturation is less than that of WT for both mutants, which is most likely caused by aggregation of the bleached mutant proteins.

Figure 3.9 - Rhodopsin meta-II decay monitored by the use of fluorescence spectroscopy

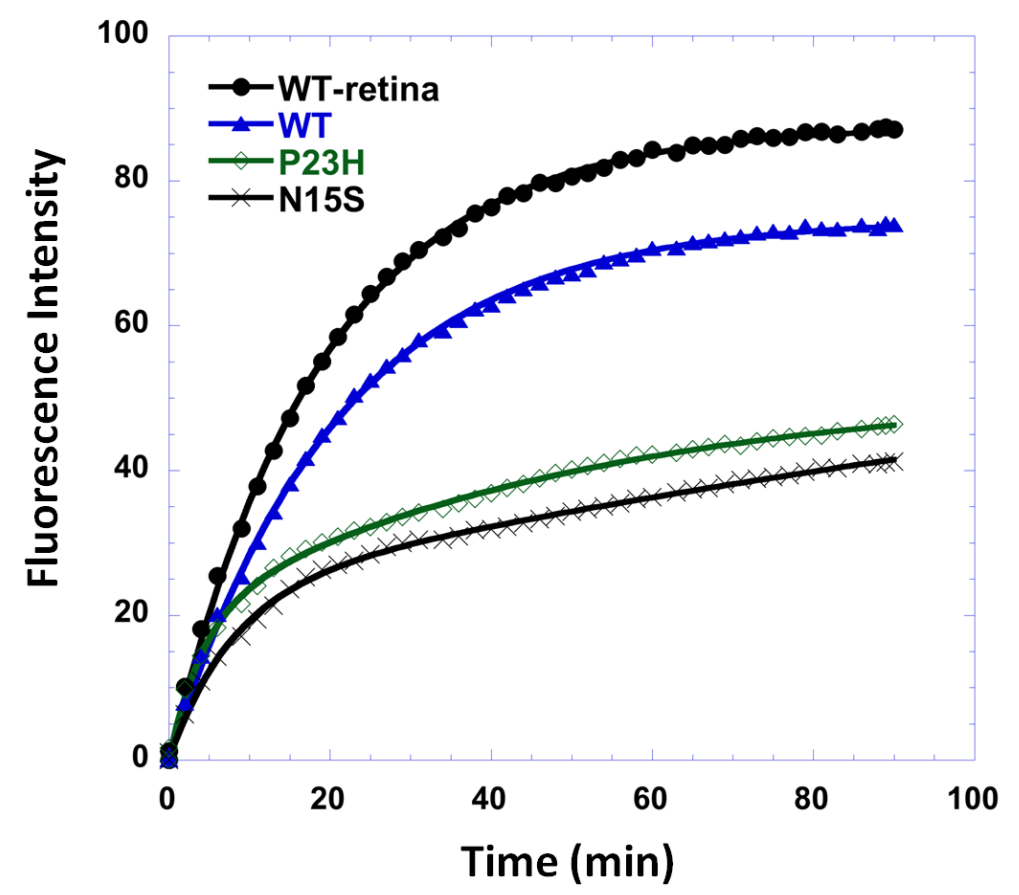

Fluorescence spectra of rhodopsin meta-II decay of rhodopsin WT purified from retina, WT, and mutants N15S and $\mathrm{P} 23 \mathrm{H}$ expressed in the presence of 9-cis retinal. Excitation and emission wavelengths $295 \mathrm{~nm}$ and $330 \mathrm{~nm}$ and slit widths of $5 \mathrm{~nm}$ and $10 \mathrm{~nm}$. The data was fitted with a double exponential rise to maximum equation using the Kaleidoscope program.

SOURCE: Balen, 2012 


\subsection{DISCUSSION}

Misfolding of rhodopsin mutants that are associated with the retinal degenerative disease ADRP has been suggested as a possible cause for the disease (Kaushal and Khorana, 1994; Sung et al., 1991, 1993). However, a direct causal relationship has not been demonstrated. It is therefore critical to better understand both the clinical as well as molecular consequences of the mutations. Previously, the molecular and clinical phenotypes of RP mutants located in different domains of rhodopsin and classified in different classes were compared (Iannaccone et al., 2006). Here, the in vitro analysis of two RP mutants, $\mathrm{P} 23 \mathrm{H}$ and $\mathrm{N} 15 \mathrm{~S}$, with same classification but different disease severity, is reported in detail, with respect to structure, folding, stability, glycosylation and in cell localization of the mutant proteins.

P23H and N15S have been shown previously to misfold (Kaushal and Khorana, 1994; Tam and Moritz, 2009). Furthermore, trafficking of human N15S expressed in Xenopus laevis was localized both at the rod outer segments and as aggregated fractions in the Golgi membranes (Tam and Moritz, 2009). P23H is also found primarily in aggresomes (Saliba et al., 2002). However, since these studies were carried out independently, a quantitative comparison was not possible. Here, we conducted all studies for the two mutants and the WT in parallel using proteins expressed in COS-1 and HEK293 cells. The results confirmed the previous observations; both mutants expressed in COS-1 cells appear to reside in aggresomes inside the cell and show signs of misfolding in vitro, including aggregation, problems in retinal-protein interactions and aberrant glycosylation. Going beyond the previous studies, we studied the possibility to rescue both mutants with retinal treatment based on previous studies with $\mathrm{P} 23 \mathrm{H}$ and other RP mutants such as T4R, T17M, P23A, P23L, and C110Y; or the nonpathogenic variants F220L and A299S (Krebs et al., 2010). Indeed, addition of 11-cis retinal during the time the protein is being synthesized to the cells prevented misfolding and helped proper trafficking of both mutants expressed in HEK293 cells. These results show the importance of 11-cis retinal in the biosynthesis and trafficking of rodopsin in cells and are in agreement with published results (Gearhart et al., 2010; Krebs et al., 2010; Maeda et al., 2006). The rescue of folded rhodopsin allowed us to carry out a larger number of in-depth biophysical characterizations of the purified mutant proteins than was previously possible. These were quantitatively compared for the two different mutants side by side. Each of these experiments consistently demonstrates that the N15S 
molecular phenotype is much milder as compared to that of P23H. FTIR suggested differences in secondary structure content between both mutants and the WT. N15S demonstrated lower levels of misfolding as judged by its overall localization on the surface of the cell. Its secondary structure resembles WT and differs from the $\mathrm{P} 23 \mathrm{H}$ mutant that presented a high degree of misfolding. N15S was more stable than $\mathrm{P} 23 \mathrm{H}$ as judged by the thermal decay rate at $37{ }^{\circ} \mathrm{C}$ that was half of that of $\mathrm{P} 23 \mathrm{H}$. Glycosylation was impaired in both mutants, however to different degrees. N15S appears normally glycosylated at the site not mutated, while $\mathrm{P} 23 \mathrm{H}$ presented higher degree of aggregation and aberrant glycosylation.

\subsection{CONCLUSIONS}

In summary, the ability to quantitatively compare the characteristics of the two mutants allowed study of the extent of severity in molecular and functional terms. The expression profiles in cell culture and molecular/biophysical in vitro studies of the purified proteins all suggest that the phenotype of N15S is misfolded but is less so than $\mathrm{P} 23 \mathrm{H}$. These molecular and cellular properties strongly correlate with patient data for these two mutants - as will be discussed in Chapter 7. 


\section{CHAPTER 4: SMALL MOLECULE BINDING TO WT RHODOPSIN PURIFIED FROM BOVINE RETINAE}

\subsection{SUMMARY}

Rhodopsin's function in vision critically depends on the binding of a small molecule ligand, 11-cis retinal, which isomerizes to all-trans retinal. Other retinal isomers are also able to bind in the same orthosteric ligand pocket. Apart from these well-established orthosteric retinal ligands, there is increasing evidence that other small molecules also bind to rhodopsin, at allosteric sites. In particular, the chlorophyll derivative chlorine e6 (Ce6) (Isayama et al., 2006; Washington et al., 2004), anthocyanin compounds, such as cyanidin-3-glucoside (C3G) (Matsumoto et al., 2003), and metal ions, in particular zinc $\left(\mathrm{Zn}^{2+}\right)$ (Park et al., 2007; Shuster et al., 1992; Stojanovic et al., 2004; Valle et al., 2003) have been implicated as putative allosteric ligands. Of these, only $\mathrm{Zn}^{2+}$ binding sites have so far been directly demonstrated (Okada et al., 2002; Palczewski et al., 2000). In the present work, thermal denaturation, circular dichroism (CD) and fluorescence spectroscopy studies, were used to systematically compare the effects of $\mathrm{Zn}^{2+}$ and other divalent cations, as well as Ce6 and C3G on rhodopsin. Specifically, detailed studies of the thermal stability of secondary and tertiary structure of rhodopsin in the presence and absence of $\mathrm{Ce} 6$ alone and together with the bivalent metal ions $\mathrm{Zn}^{2+}, \mathrm{Cu}^{2+}, \mathrm{Fe}^{2+}, \mathrm{Ni}^{2+}, \mathrm{Mg}^{2+}$ and $\mathrm{Mn}^{2+}$ over a temperature range from $5{ }^{\circ} \mathrm{C}$ to $100{ }^{\circ} \mathrm{C}$ were conducted using circular dichroism and fluorescence spectroscopy. A pronounced increase in the thermal stability of the overall secondary structure content of rhodopsin is observed when both $\mathrm{Ce} 6$ and $\mathrm{Zn}^{2+}$ are present, as compared to rhodopsin with either compound alone. This thermal stability increase is also noted with $\mathrm{Cu}^{2+}$, but not when other metal ions and Ce6 are combined. Similar studies conducted in the presence of $\mathrm{C} 3 \mathrm{G}$ support the notion that $\mathrm{C} 3 \mathrm{G}$ exerts a destabilizing effect on rhodopsin structure. It modestly alters the rates at which the light-activated Metarhodopsin II state decays to opsin and free retinal. These results indicate that the mechanism of $\mathrm{C} 3 \mathrm{G}$ enhanced regeneration may be based on changes in rhodopsin structure upon illumination, promoting access to the retinal binding pocket. In contrast, binding of $\mathrm{Ce} 6$ and divalent metal ions keep the helical bundle closed, preventing access to the retinal binding pocket. 


\subsection{INTRODUCTION}

Several studies have aimed at elucidating the molecular consequences of $\mathrm{Zn}^{2+}$ binding to rhodopsin with considerable dissent about the question whether $\mathrm{Zn}^{2+}$ has stabilizing or destabilizing effects on rhodopsin (Park et al., 2007; Stojanovic et al., 2004; Valle et al., 2003). It has been found that $\mathrm{Zn}^{2+}$ alters the rate at which retinal leaves the rhodopsin binding pocket at elevated temperatures, which appears to be predominantly increased in the presence of added $\mathrm{Zn}^{2+}$ (Stojanovic et al., 2004; Valle et al., 2003), denoting a destabilizing effect. Single molecule atomic force microscopy (AFM) measurements have shown that the presence of $\mathrm{Zn}^{2+}$ did not affect the location of stable structural segments of rhodopsin, but increased the force necessary to unfold the segments (Park et al., 2007). This result suggests that $\mathrm{Zn}^{2+}$ stabilizes the overall structure of the protein. Furthermore, $\mathrm{Zn}^{2+}$ enhances the extent of rhodopsin phosphorylation (Shuster et al., 1996) and slightly inhibits G protein activation (Sheikh et al., 1996).

In Chapter 1, it was shown that there are other potential ligands for rhodopsin, such as anthocyanins $(\mathrm{C} 3 \mathrm{G})$ and porphyrins (Ce6). Regeneration studies showed that $\mathrm{C} 3 \mathrm{G}$ and other anthocyanins are able to enhance the regeneration of rhodopsin (Matsumoto et al., 2003, 2006). Ce6 and other phorphyrins may act as photosensitizers for detection of red light to which rhodopsin is normally not very sensitive (Douglas et al., 1998, 1999; Isayama et al., 2006; Washington et al., 2004, 2007). In vivo studies have shown that Ce6 successfully enhances vision in different animals (Isayama et al., 2006; Washington et al., 2007). In parallel to my thesis work, further evidence was gathered supporting the notion that both Ce6 and C3G physically bind to rhodopsin and modulate its structure and function in vitro (Yanamala, 2009).

In this thesis, I aimed to stratify the discrepancy on whether the effect of $\mathrm{Zn}^{2+}$ is stabilizing or destabilizing. To this end, biophysical measurements of thermal stability of secondary and tertiary structure in the presence and absence of the bivalent metal ions, $\mathrm{Zn}^{2+}$, $\mathrm{Cu}^{2+}, \mathrm{Fe}^{2+}, \mathrm{Ni}^{2+}, \mathrm{Mg}^{2+}$ and $\mathrm{Mn}^{2+}$ were systematically compared. I extended these studies to the other putative ligands, $\mathrm{Ce} 6$ and $\mathrm{C} 3 \mathrm{G}$, and tested whether there are additive or synergistic effects of multiple ligands binding simultaneously. 


\subsubsection{Ce6 interaction with rhodopsin}

\subsubsection{Effects of Ce6 binding on secondary structure of rhodopsin}

As a first step, the effects of Ce6 on the stability of secondary structure of rhodopsin were studied through direct biophysical measurements. Thermal stability experiments were performed, where the temperature was varied from $5{ }^{\circ} \mathrm{C}$ to $100{ }^{\circ} \mathrm{C}$. Rhodopsin secondary structure loss was monitored over this temperature range using $\mathrm{CD}$ spectroscopy in $5{ }^{\circ} \mathrm{C}$ intervals. The total time of the experiment was approximately 4 hours. Representative CD spectra are shown for rhodopsin alone (Figure 4.1A), and for rhodopsin combined with Ce6 (Figure 4.1B). The mean residue elipticity (MRE) at $222 \mathrm{~nm}$ is characteristic of $\alpha$-helix, and the MRE values at $222 \mathrm{~nm}$ extracted from the full-range spectra shown in Figure 4.1A and B are normalized and plotted in Figure 4.1C to allow comparison between presence and absence of Ce6. Quantitative analysis of the curves from Figure 4.1C is provided in Table 4.2. The maximal percent decrease in MRE at 222 $\mathrm{nm}$ is reported. Even though these numbers are qualitative, for reference, the minimum $\alpha$-helix content remaining at the end of the thermal cycle, and the mid-point transition temperatures values were calculated by fitting the normalized $\alpha$-helix content data to a nonlinear logistic 4parameter regression function (Materials and Methods, Chapter 2).

The effect of Ce6 (Figure 4.1C) is to shift the temperature at which rhodopsin unfolds to higher values. Furthermore, the end-point of the transition at $100^{\circ} \mathrm{C}$ is reduced; the decrease in the MRE at $222 \mathrm{~nm}$ in the presence of Ce6 is $\sim 40 \%$, where in the absence it is $\sim 50 \%$. Using CDPro to deconvolute the spectra, this decrease is estimated to correspond to a $\sim 40 \%$ loss of helix content as compared to $\sim 60 \%$ in the absence of $\mathrm{Ce} 6$, qualitatively indicating a stabilization of helix structure by Ce6. 
Figure 4.1 - Thermal denaturation studies of rhodopsin in the presence of Chlorin e6 (Ce6) using circular dichroism.
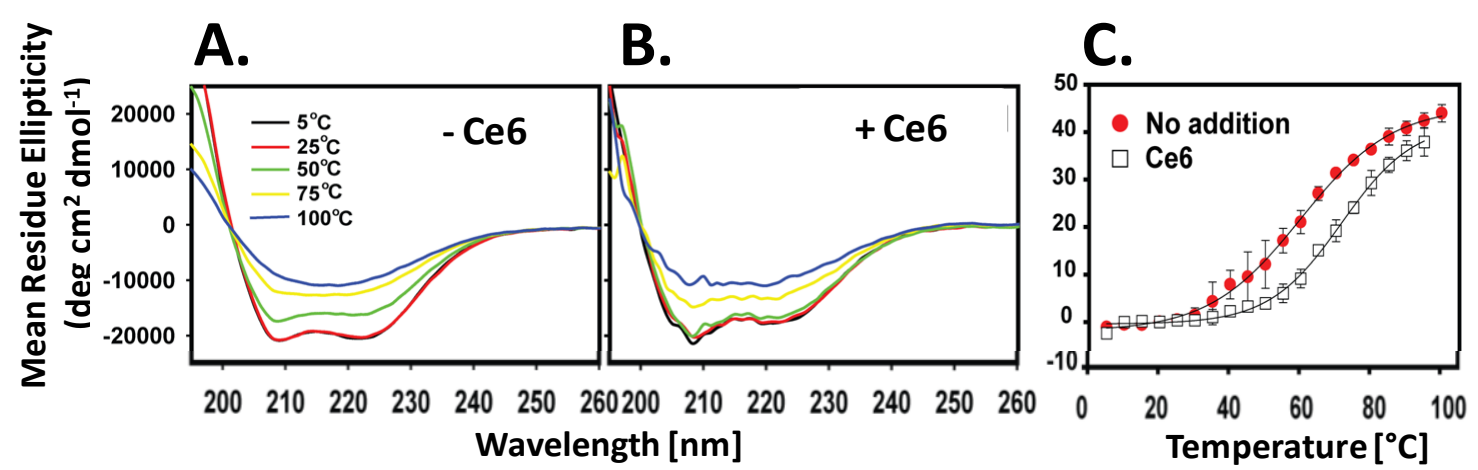

CD melting spectra of rhodopsin $(2.5 \mu \mathrm{M})$. B. CD melting spectra of rhodopsin $(2.5 \mu \mathrm{M})$ in the presence of Ce6 $(100 \mu \mathrm{M})$. C. Percent decrease in mean residue ellipticity at $222 \mathrm{~nm}$ in the presence and absence of Ce6 as a function of temperature. White square: rhodopsin + Ce6. Red circle: rhodopsin alone.

SOURCE: Figure is adapted from (Balen, 2009). With permission.

\subsubsection{Effects of Ce6 binding on tertiary structure of rhodopsin}

To better understand the structural transitions occurring during thermal denaturation of rhodopsin, intrinsic tryptophan fluorescence was measured as a function of temperature. The fluorescence traces of rhodopsin were recorded by increasing the temperature gradually from $5{ }^{\circ} \mathrm{C}$ to $100{ }^{\circ} \mathrm{C}$ with and without $\mathrm{Ce} 6$ (Figure 4.2). There are two major transitions resulting in increases in tryptophan fluorescence, one occurring at around $20{ }^{\circ} \mathrm{C}$ to $40{ }^{\circ} \mathrm{C}$ and another, at around $50{ }^{\circ} \mathrm{C}$ to $70{ }^{\circ} \mathrm{C}$. The presence of $\mathrm{Ce} 6$ quenches rhodopsin tryptophan fluorescence, indicating that the interaction between rhodopsin and Ce6 is stable over the course of the experiment and that $\mathrm{Ce} 6$ remains bound, even at the highest temperature measured. 
Figure 4.2 - Thermal melting curves of rhodopsin.

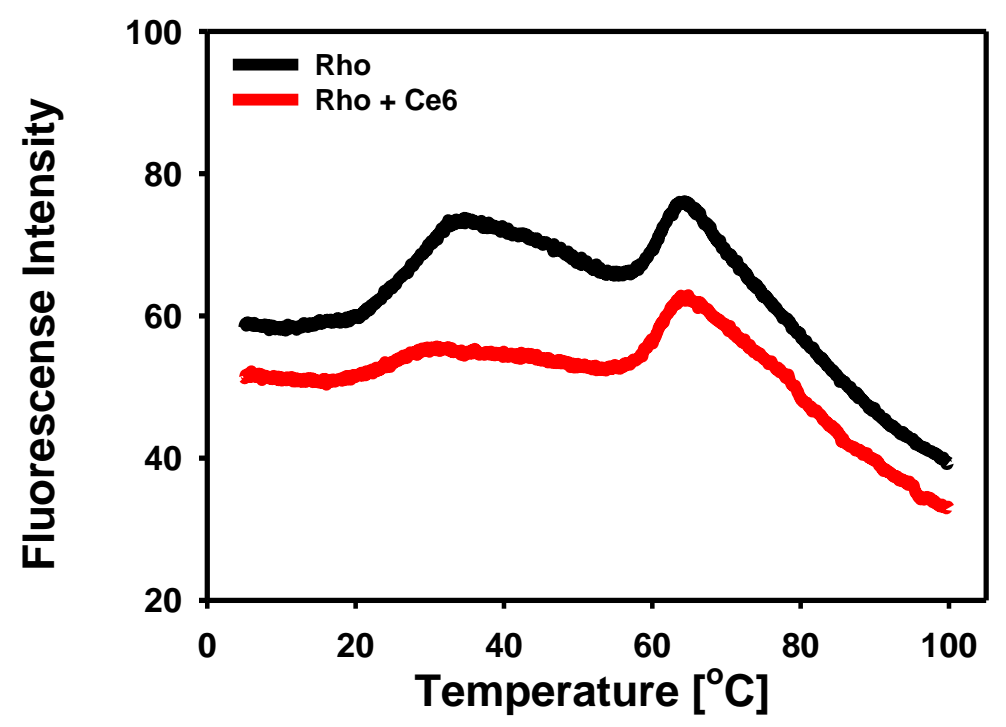

Thermal denaturation measured using fluorescence spectroscopy. Samples were placed in a cuvette holder and the temperature was constantly increased from $5{ }^{\circ}$ to $100{ }^{\circ} \mathrm{C}$. Fluorescence traces were obtained at an excitation wavelength of $280 \mathrm{~nm}$, and emission wavelength of $330 \mathrm{~nm}$ and slit widths of $5 \mathrm{~nm}$ and $10 \mathrm{~nm}$. Black line: rhodopsin alone. Red line: rhodopsin in the presence of Ce6. A concentration of $2.5 \mu \mathrm{M}$ for rhodopsin and $1.25 \mu \mathrm{M}$ of Ce6 were used.

SOURCE: Figure is adapted from (Tirupula, 2009). With permission.

Tryptophan fluorescence was also recorded as a function of time at $55{ }^{\circ} \mathrm{C}$ (Figure 4.3). The increase in temperature causes thermally activated retinal release, which requires breakage of non-covalent tertiary interactions between the protein and the retinal, and hydrolysis of the retinal Schiff base which covalently anchors the retinal to Lys296. After retinal has left, tryptophan fluorescence can be quenched independently of the retinal if the protein retains some level of compact structure resulting in protein-based quenching. Only if the protein is fully unfolded, will one obtain the full maximum of fluorescence that one would obtain with free tryptophan. Thus, there are multiple processes - retinal dependent and independent ones - that are induced by increasing the temperature. All of these processes are tertiary-contact dependent, therefore fluorescence spectroscopy was applied here as a qualitative indicator for changes in tertiary interactions. Rhodopsin in the presence of Ce6 shows a small increase in response to the temperature change and no visible change upon illumination, while an increase in fluorescence is observed in the absence of $\mathrm{Ce} 6$ in response to temperature change and light illumination. 
Figure 4.3 - Effects of Chlorin e6 (Ce6) on thermal stability of rhodopsin at $55^{\circ} \mathrm{C}$ using fluorescence spectroscopy.

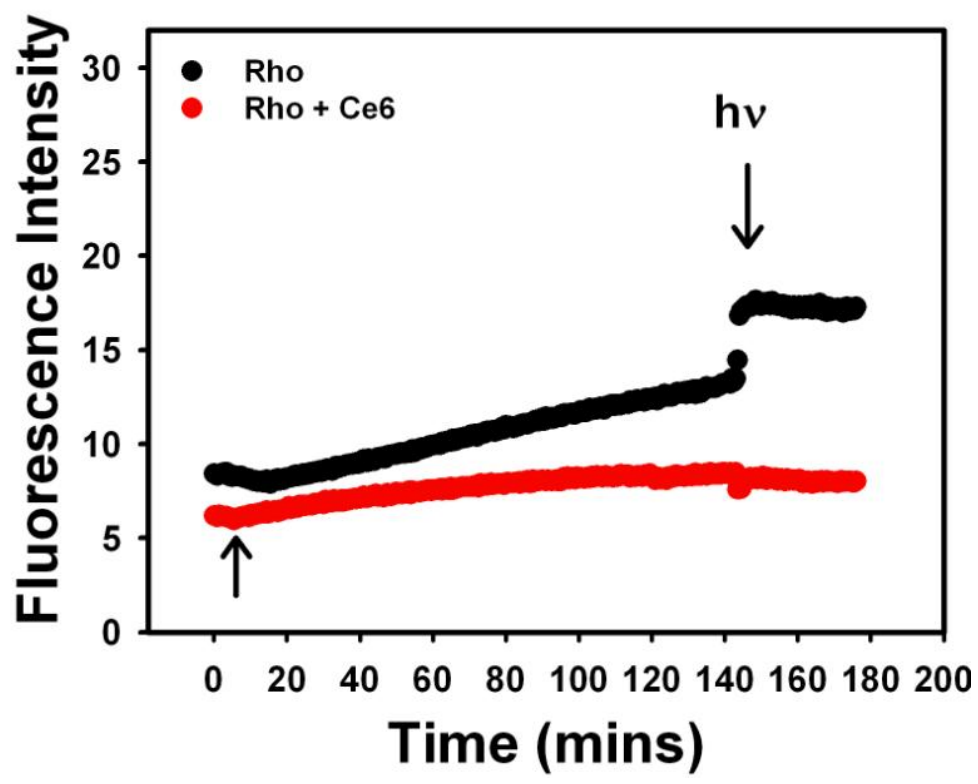

Concentrations used were: Rhodopsin $0.5 \mu \mathrm{M}$ and Ce6 $1.25 \mu \mathrm{M}$. An excitation slit-width of $5 \mathrm{~nm}$ and emission slitwidth of $5 \mathrm{~nm}$ was used to record the fluorescence traces. Samples were first recorded at $20{ }^{\circ} \mathrm{C}$ for 5 minutes and then the temperature was changed to $55{ }^{\circ} \mathrm{C}$ (indicated by an upward arrow). After 140 minutes the samples were illuminated using a $>495 \mathrm{~nm}$ light source (indicated by a downward arrow).

SOURCE: Balen, 2012

\subsubsection{Effect of Ce6 binding on the Meta II decay of rhodopsin}

To investigate whether the stability of Meta II itself is changed, its decay to free retinal and opsin at $20{ }^{\circ} \mathrm{C}$ using fluorescence spectroscopy was measured. There are five tryptophan residues in rhodopsin and their fluorescence to a large extent is quenched in the dark. Upon illumination, an increase in fluorescence is observed over time as a result of the retinal leaving the binding pocket (Farrens and Khorana, 1995). Figure 4.4 shows that the fluorescence is quenched in the dark as well in the light-activated state, indicating the binding of Ce6 and rhodopsin. Meta II decay half-lives of rhodopsin in the absence and presence of Ce6 at various concentrations are shown in Table 4.1. Based on the $t_{1 / 2}$ values it was found that rhodopsin tryptophan fluorescence is strongly quenched by $\mathrm{Ce} 6$, especially in the light. 
Figure 4.4 - Effects on rhodopsin Meta II determined by fluorescence spectroscopy.

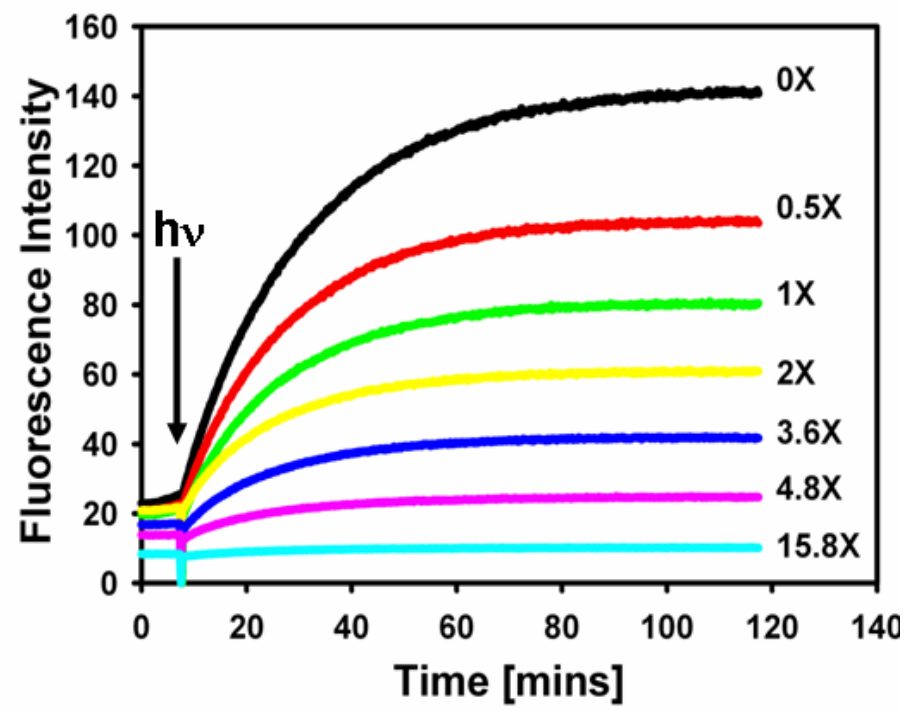

Samples were first recorded at $20{ }^{\circ} \mathrm{C}$ then illuminated for 60 seconds (indicated by the downward arrow). Fluorescence traces obtained at wavelengths for excitation of $280 \mathrm{~nm}$ and for emission of $330 \mathrm{~nm}$ in the presence of different concentrations of Ce6. Slit widths of $5 \mathrm{~nm}$ and $10 \mathrm{~nm}$. Rhodopsin concentration: $0.5 \mu \mathrm{M}$.

SOURCE: Cortesy of Naveena Yanamala (Yanamala, 2009).

Table 4.1 - Meta II decay half-lives of rhodopsin in the absence and presence of Ce6 at various concentrations indicated.

\begin{tabular}{cc}
\hline Ce6 Concentration & Meta II decay Half-life \\
\hline $0.0 \mu \mathrm{M}$ & $13.51 \pm 0.3$ \\
$0.5 \mu \mathrm{M}$ & $12.86 \pm 0.76$ \\
$1.0 \mu \mathrm{M}$ & $12.47 \pm 0.75$ \\
$1.8 \mu \mathrm{M}$ & $12.28 \pm 0.78$ \\
$2.4 \mu \mathrm{M}$ & $11.57 \pm 2.81$ \\
$7.93 \mu \mathrm{M}$ & Cannot be estimated \\
\hline
\end{tabular}

All samples contained a rhodopsin concentration of $0.5 \mu \mathrm{M}$.

SOURCE: Balen, 2012 
4.2.2 Metal ion effects on rhodopsin in the presence and absence of Ce6

\subsubsection{Effects of Ce6 and metal ion binding on secondary structure of rhodopsin}

In order to study the effects of metal ions on the secondary structure of rhodopsin, thermal stability experiments were performed, using CD spectroscopy as described above in Section 4.2.1.1. The divalent metal ions compared were $\mathrm{Zn}^{2+}, \mathrm{Cu}^{2+}, \mathrm{Fe}^{2+}, \mathrm{Mn}^{2+}, \mathrm{Mg}^{2+}$ and $\mathrm{Ni}^{2+}$. Representative CD spectra are shown for rhodopsin alone (Figure 4.5A, panel I), rhodopsin plus Ce6 (Figure 4.5B, panel I), and in the presence of divalent metal-ions without (Figure 4.5A, panels II-IV) and with Ce6 (Figure 4.5B, panels II-IV). The mean residue ellipticity (MRE) at $222 \mathrm{~nm}$ is particularly characteristic for $\alpha$-helix, and the MRE values extracted from the spectra depicted in Figure 4.5A and B are shown in Figure 4.5C to allow comparison of the different buffer conditions. Quantitative analysis of the curves shown in Figure 4.5C is provided in Table 4.2. The maximal percent decrease in the MRE at $222 \mathrm{~nm}$ is reported. Increasing the temperature from $5{ }^{\circ} \mathrm{C}$ to $100{ }^{\circ} \mathrm{C}$ resulted in a general decrease in magnitude of MRE at $222 \mathrm{~nm}$, which is indicative of an overall loss of secondary structure content. Rhodopsin in the absence of any additives is shown in all panels for reference as red circles. 
Figure 4.5 - Thermal denaturation studies of rhodopsin in the presence of different metal ions and Ce6 using circular dichroism.
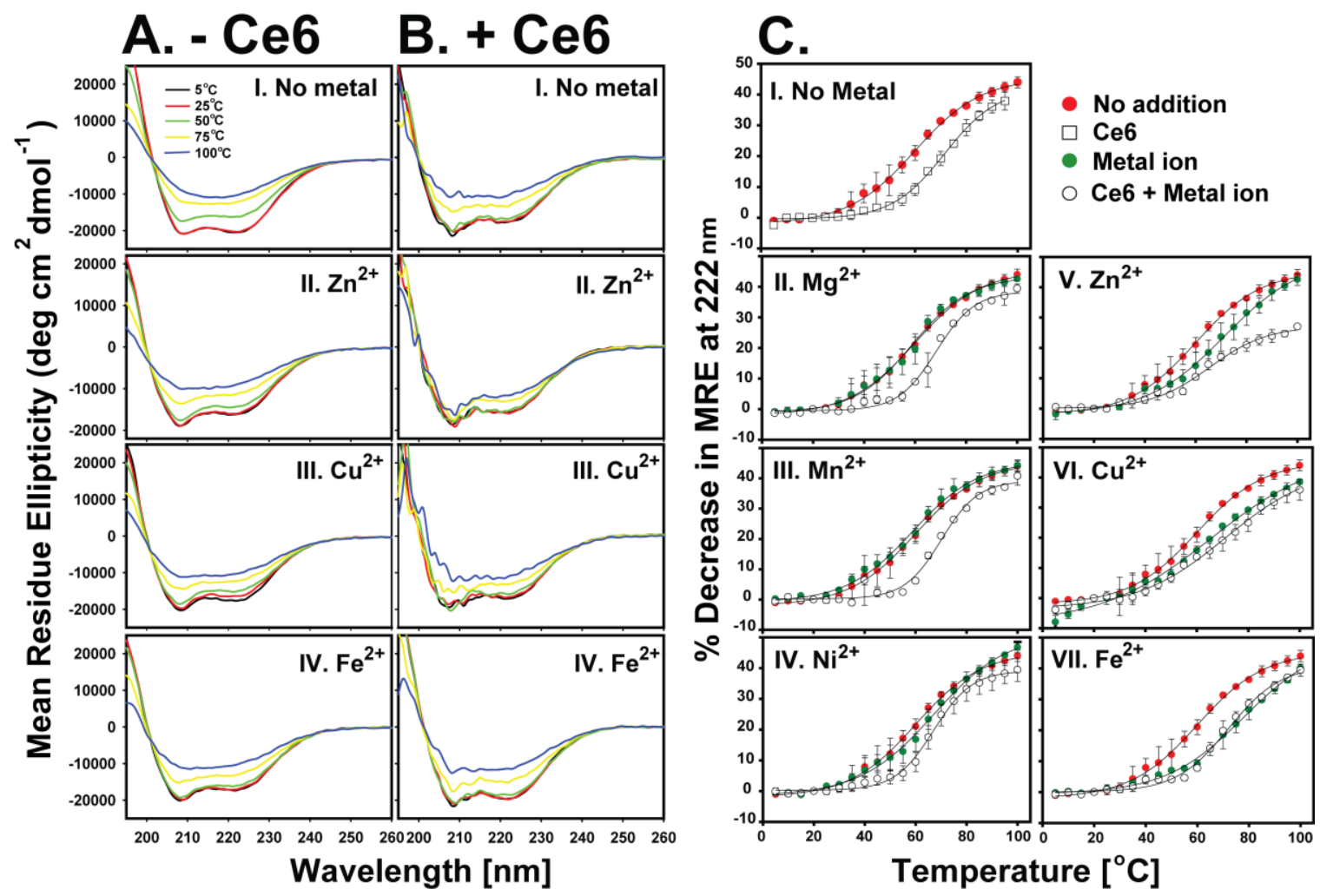

A. CD melting spectra of rhodopsin $(2.5 \mu \mathrm{M})$ in the presence of divalent metals. The concentration of divalent metals was $100 \mu \mathrm{M}$. Panel I, no metal added. Panel II, in the presence of $\mathrm{Zn}^{2+}$. Panel III, in the presence of $\mathrm{Cu}^{2+}$. Panel IV, in the presence of $\mathrm{Fe}^{2+}$. B. CD melting spectra of rhodopsin $(2.5 \mu \mathrm{M})$ in the presence of Ce6 $(100 \mu \mathrm{M})$ and divalent metals $(100 \mu \mathrm{M})$. Panels as in A. C. Percent decrease in mean residue ellipticity at $222 \mathrm{~nm}$ in the presence and absence of divalent metal ions and Ce6 as a function of temperature. White square: rhodopsin + Ce6. Circles: red: rhodopsin alone; green: rhodopsin + metal ion; white: rhodopsin + $\mathrm{Ce} 6+$ metal ion. Panel I, no metal added. Panel II, in the presence of $\mathrm{Mg}^{2+}$. Panel III, in the presence of $\mathrm{Mn}^{2+}$. Panel IV, in the presence of $\mathrm{Ni}^{2+}$. Panel V, in the presence of $\mathrm{Zn}^{2+}$. Panel VI, in the presence of $\mathrm{Cu}^{2+}$. Panel VII, in the presence of $\mathrm{Fe}^{2+}$.

SOURCE: Figure is adapted from (Balen, 2009). With permission.

The effect of bivalent metal ions was dependent on the chemical nature of the ion studied. Each metal ion displayed a stabilizing effect on rhodopsin secondary structure, as evidenced by the increase of the MRE at $222 \mathrm{~nm}$ and also either by a shift of the transition mid-point to higher temperatures or by an increase in final $\alpha$-helix content. The most significant changes were observed with $\mathrm{Zn}^{2+}, \mathrm{Fe}^{2+}$ and $\mathrm{Cu}^{2+}$. In the case of $\mathrm{Zn}^{2+}$ and $\mathrm{Fe}^{2+}$, the midpoint of the transition was shifted from $74 \pm 3{ }^{\circ} \mathrm{C}$ for rhodopsin alone to $83 \pm 3{ }^{\circ} \mathrm{C}$ in the presence of $\mathrm{Zn}^{2+}$ and $84 \pm 3{ }^{\circ} \mathrm{C}$ in the presence of $\mathrm{Fe}^{2+}$. In the case of $\mathrm{Cu}^{2+}$, the decrease in absolute MRE magnitude was only $39 \%$ as compared with rhodopsin alone, which was $50 \%$. 
The presence of Ce6 in each case further enhances the stabilizing effects provided by the metal ions alone. Table 4.2 shows that in the presence of Ce6 the decrease in MRE at $222 \mathrm{~nm}$ was in general smaller. Furthermore, the estimated $\alpha$-helix content left at the end of the thermal denaturation $\left(100{ }^{\circ} \mathrm{C}\right)$ in the presence of $\mathrm{Ce} 6$ is about $1.3-2.4$ fold higher as compared to the rhodopsin controls containing only the metal ions (with the exception of $\mathrm{Mg}^{2+}$, where the minimum $\alpha$-helix content remained comparable). The maximum difference was observed for $\mathrm{Zn}^{2+}$. The decrease in the magnitude of MRE at $222 \mathrm{~nm}$ was $43 \%$ for $\mathrm{Zn}^{2+}$ alone and $27 \%$ in the presence of $\mathrm{Ce} 6$ and $\mathrm{Zn}^{2+}$. Furthermore, the estimated minimum $\alpha$-helix content increased 2.2 fold from 30 to 65 when $\mathrm{Zn}^{2+}$ and $\mathrm{Ce} 6$ were present together as compared to $\mathrm{Zn}^{2+}$ alone. The mid-point of the transition was shifted to such high temperatures that it could not be determined accurately (Figure $4.5 \mathrm{C}$, panel V). In conclusion, $\mathrm{Zn}^{2+}$ and $\mathrm{Ce} 6$ is the best combination to stabilize overall secondary structure of rhodopsin. 
Table 4.2 - Percent decrease in mean residue ellipticity at $222 \mathrm{~nm}$ at $100{ }^{\circ} \mathrm{C}$, minimum $\alpha$-helix content present at $100{ }^{\circ} \mathrm{C}$ after thermal denaturation, and midpoint transition temperatures of rhodopsin in the presence of different metal ions and Ce6.

\begin{tabular}{|c|c|c|c|}
\hline & $\begin{array}{c}\text { Percent } \\
\text { decrease in } \\
\text { MRE at } \\
222_{\mathrm{nm}}\end{array}$ & $\begin{array}{l}\text { Minimum } \\
\text { helix } \\
\text { content }\end{array}$ & $\begin{array}{c}\text { Midpoint of } \\
\text { transition }\end{array}$ \\
\hline Rho & $50 \pm 2$ & $25 \pm 5$ & $74 \pm 3$ \\
\hline Rho + Ce6 & $40 \pm 3$ & $58 \pm 1$ & $73 \pm 0$ \\
\hline $\mathrm{Rho}+\mathrm{Zn}^{2+}$ & $43 \pm 2$ & $30 \pm 8$ & $83 \pm 3$ \\
\hline $\mathrm{Rho}+\mathrm{Ce} 6+\mathrm{Zn}^{2+}$ & $27 \pm 1$ & $65 \pm \mathrm{ND}$ & ND \\
\hline $\mathrm{Rho}+\mathrm{Fe}^{2+}$ & $40 \pm 0$ & $39 \pm 6$ & $84 \pm 3$ \\
\hline $\mathrm{Rho}+\mathrm{Ce} 6+\mathrm{Fe}^{2+}$ & $39 \pm 2$ & $56 \pm 4$ & $80 \pm 2$ \\
\hline $\mathrm{Rho}+\mathrm{Cu}^{2+}$ & $39 \pm 1$ & $51 \pm 2$ & $72 \pm 1$ \\
\hline $\mathrm{Rho}+\mathrm{Ce} 6+\mathrm{Cu}^{2+}$ & $36 \pm 4$ & $73 \pm 3$ & $71 \pm 3$ \\
\hline $\mathrm{Rho}+\mathrm{Ni}^{2+}$ & $47 \pm 2$ & $40 \pm 3$ & $73 \pm 2$ \\
\hline $\mathrm{Rho}+\mathrm{Ce} 6+\mathrm{Ni}^{2+}$ & $39 \pm 4$ & $59 \pm 3$ & $70 \pm 2$ \\
\hline $\mathrm{Rho}+\mathrm{Mg}^{2+}$ & $46 \pm 1$ & $41 \pm 3$ & $69 \pm 2$ \\
\hline $\mathrm{Rho}+\mathrm{Ce} 6+\mathrm{Mg}^{2+}$ & $39 \pm 2$ & $43 \pm 7$ & $77 \pm 4$ \\
\hline $\mathrm{Rho}+\mathrm{Mn}^{2+}$ & $44 \pm 2$ & $39 \pm 4$ & $69 \pm 2$ \\
\hline $\mathrm{Rho}+\mathrm{Ce} 6+\mathrm{Mn}^{2+}$ & $41 \pm 3$ & $65 \pm 4$ & $69 \pm 3$ \\
\hline
\end{tabular}

SOURCE: Table is adapted from (Balen, 2009). With permission.

While the thermal denaturation experiments reported above serve to qualitatively differentiate the ability of different ligands to stabilize rhodopsin, they cannot be used to extract quantitative thermodynamic information. This is shown in Figure 4.6, where it is clear that the 
thermal transitions recorded are irreversible. The figure shows thermal denaturation of secondary structure $\left(5-100{ }^{\circ} \mathrm{C}\right)$ and putative renaturation $\left(100-5^{\circ} \mathrm{C}\right)$ using $\mathrm{CD}$ spectroscopy. The MRE at $222 \mathrm{~nm}$ of the denaturation (filled symbols) and renaturation (open symbols) cycles in the absence (triangles) and presence (circles) of both $\mathrm{Zn}^{2+}$ and $\mathrm{Ce} 6$ are shown. As mentioned above, thermal denaturation results in a decrease in absolute MRE at $222 \mathrm{~nm}$. Attempts to refold rhodopsin, showed a further decrease in the MRE in the absence of additives. In contrast, almost no changes were observed in the presence of $\mathrm{Ce} 6$ and $\mathrm{Zn}^{2+}$. While this is an improvement over the cold-denaturation (further decrease in helicity when going back from $100{ }^{\circ} \mathrm{C}$ to $5{ }^{\circ} \mathrm{C}$ ) observed in the absence of additives, it does not constitute a reversible transition. In both cases, thermal denaturation presumably leads to irreversible misfolding of the protein in DM, from which the folded state cannot be recovered.

Figure 4.6 - Thermal melting curves of rhodopsin.

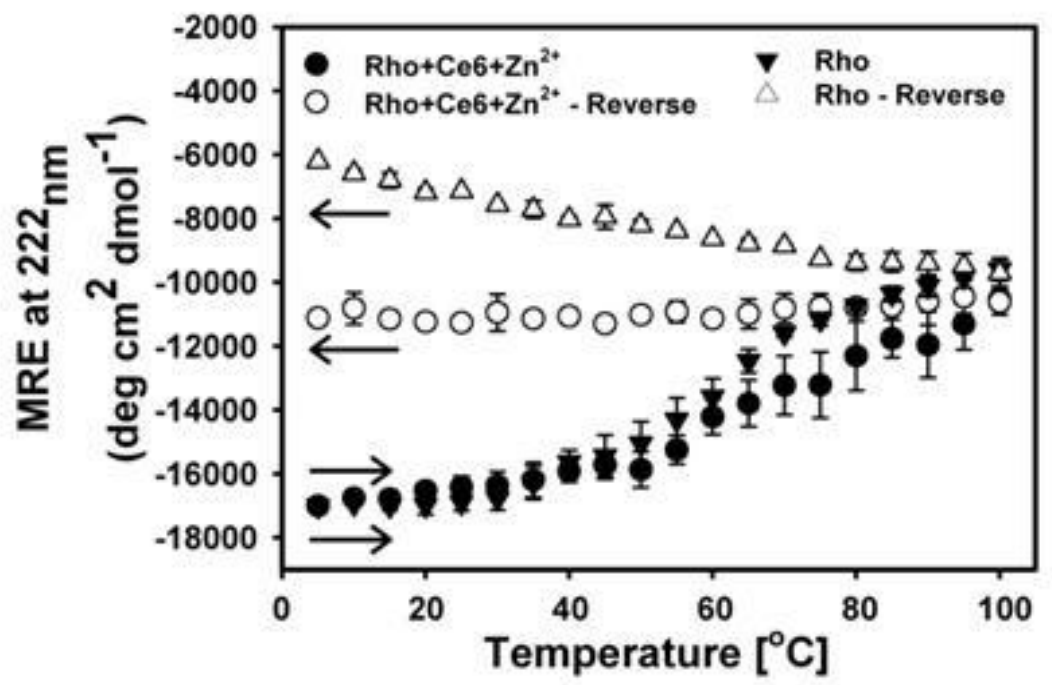

Thermal denaturation measured by circular dichroism. Closed symbols are for heating cycle $\left(5{ }^{\circ} \mathrm{C}-100{ }^{\circ} \mathrm{C}\right)$. Open symbols are for cooling cycle $\left(100{ }^{\circ} \mathrm{C}-5{ }^{\circ} \mathrm{C}\right)$. Triangles: rhodopsin alone. Circles: rhodopsin, in the presence of Ce6 and $\mathrm{Zn}^{2+}$. A concentration of $2.5 \mu \mathrm{M}$ for rhodopsin, $100 \mu \mathrm{M}$ for $\mathrm{Zn}^{2+}$ and $100 \mu \mathrm{M}$ for $\mathrm{Ce} 6$ was used.

SOURCE: Figure is adapted from (Balen, 2009). With permission. 


\subsubsection{Effects of Ce6 binding on tertiary structure of rhodopsin}

Previous studies have shown that the rate of retinal leaving the protein at elevated temperature $\left(55^{\circ} \mathrm{C}\right.$ ) was enhanced in the presence of exogenously added $\mathrm{Zn}^{2+}$ (Stojanovic et al., 2004; Valle et al., 2003). The thermally induced disruption of retinal-chromophore interactions can be measured as a function of time, by following the loss in $500 \mathrm{~nm}$ chromophore and appearance of a $380 \mathrm{~nm}$ peak, which represents free retinal in absorbance spectra of rhodopsin. To ensure consistency of the experiments with these previous studies, constant temperature thermal chromophore decay of rhodopsin was recorded with and without $\mathrm{Zn}^{2+}$ at $55{ }^{\circ} \mathrm{C}$ by UV/Vis absorbance spectroscopy (Figure 4.7). A decay rate of 0.025 minutes $^{-1}$ and 0.104 minutes ${ }^{-1}$ was observed for rhodopsin in the absence and presence of $\mathrm{Zn}^{2+}$, respectively. The corresponding $\mathrm{t}_{1 / 2}$ values calculated are 27.0 minutes for rhodopsin alone and 6.6 minutes for rhodopsin in the presence of $\mathrm{Zn}^{2+}$. These results are in good agreement with those reported previously (Stojanovic et al., 2004; Valle et al., 2003).

Figure 4.7 - Effects of $\mathrm{Zn}^{2+}$ binding on chromophore stability.

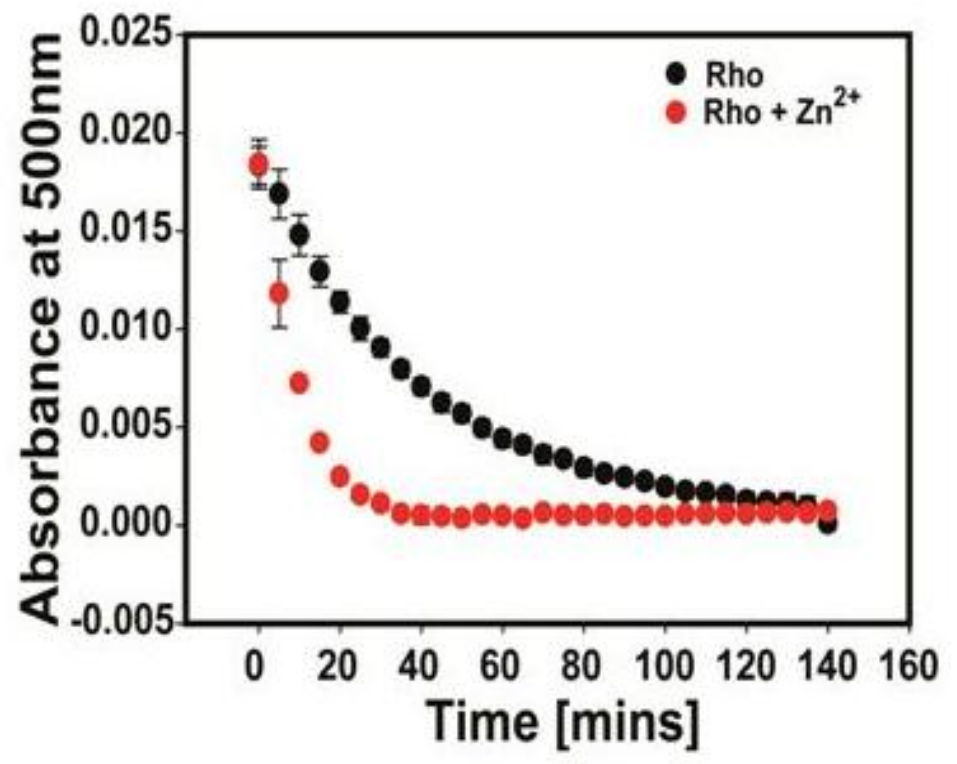

Thermal stability of rhodopsin in the absence (black circles) and presence of $\mathrm{Zn}^{2+}$ (red circles), at $55^{\circ} \mathrm{C}$. Stability of rhodopsin was measured as a function of the decay of the absorbance at $500 \mathrm{~nm}$ over time.

SOURCE: Figure is adapted from (Balen, 2009). With permission. 
To better understand the structural transitions occurring during thermal denaturation of rhodopsin, intrinsic tryptophan fluorescence was measured as a function of temperature. The fluorescence traces of rhodopsin were recorded by increasing the temperature gradually from 5 ${ }^{\circ} \mathrm{C}$ to $100{ }^{\circ} \mathrm{C}$ with and without $\mathrm{Zn}^{2+}$ and $\mathrm{Ce} 6$, and both of them together (Figure 4.8). There are two major transitions that result in increases in tryptophan fluorescence, one occurring around 20 ${ }^{\circ} \mathrm{C}$ to $40{ }^{\circ} \mathrm{C}$ and another around $50{ }^{\circ}$ to $70^{\circ} \mathrm{C}$ as described above (Section 4.2.1.2). The first transition was essentially unaffected by $\mathrm{Zn}^{2+}$. In contrast, the increase in the tryptophan fluorescence of the second transition state was much higher in the presence of $\mathrm{Zn}^{2+}$ as compared to rhodopsin alone. This indicates an opening of the helical bundle and/or increased rate of retinal-protein dissociation by $\mathrm{Zn}^{2+}$ at high temperatures. The presence of Ce6 quenches rhodopsin tryptophan fluorescence, both in the presence and absence of $\mathrm{Zn}^{2+}$ (Figure 4.8, yellow and green lines, respectively), indicating that the interaction between rhodopsin and Ce6 is stable over the course of the experiment and Ce6 remains bound, even at the highest temperatures. Because of the quenching of tryptophan fluorescence by $\mathrm{Ce} 6$, it is not possible to ascertain whether or not the changes induced in rhodopsin structure by $\mathrm{Zn}^{2+}$ are altered by the presence of Ce6. 
Figure 4.8 - Thermal denaturation measured using fluorescence spectroscopy.

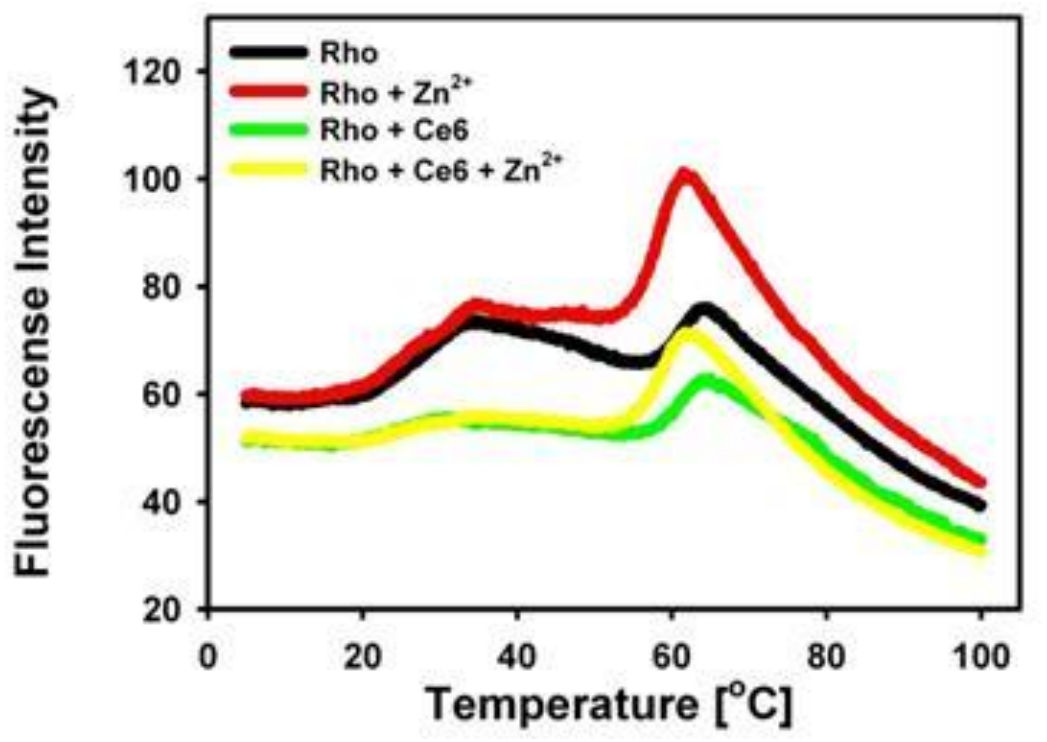

Fluorescence traces were obtained at an excitation wavelength of $280 \mathrm{~nm}$, and emission wavelength of $330 \mathrm{~nm}$. Slit widths of $5 \mathrm{~nm}$ and $10 \mathrm{~nm}$. Black line: rhodopsin alone. Red: rhodopsin, in the presence of $\mathrm{Zn}^{2+}$. Green: rhodopsin, in the presence of Ce6. Yellow: rhodopsin, in the presence of $\mathrm{Ce} 6$ and $\mathrm{Zn}^{2+}$. A concentration of $2.5 \mu \mathrm{M}$ for rhodopsin and $100 \mu \mathrm{M}$ for $\mathrm{Zn}^{2+}$ was used. A Ce6 concentration of $1.25 \mu \mathrm{M}$ was used.

SOURCE: Balen, 2012

Finally, to allow comparison of fluorescence data with the results from absorbance spectroscopy shown in Figure 4.7, tryptophan fluorescence was recorded as a function of time at $55{ }^{\circ} \mathrm{C}$ (Figure 4.9). The fluorescence increase with time was dramatically enhanced by the presence of $\mathrm{Zn}^{2+}$. This, in part, can be explained by the enhanced rate of retinal release suggested also by absorbance spectroscopy. However, the changes in fluorescence and absorbance occur on different time scales. For example, in the presence of $\mathrm{Zn}^{2+}$, there is no further decrease in $500 \mathrm{~nm}$ absorbance after 40 minutes, while the fluorescence is still increasing. Furthermore, after 140 minutes, there is a small fraction of rhodopsin that is resistant to thermal decay: when illuminated (Figure 4.9 downward arrow), a change in tryptophan fluorescence was observed, indicating that a small amount of retinal is still bound to rhodopsin. Remarkably, in the presence of $\mathrm{Zn}^{2+}$ (Figure 4.9, red and yellow traces), there is a decrease in fluorescence upon illumination, while in the absence of $\mathrm{Zn}^{2+}$, an increase in fluorescence is observed. This finding and the differences in kinetics between fluorescence and absorbance spectroscopy support the notion that $\mathrm{Zn}^{2+}$ not only alters retinal dissociation kinetics but also rhodopsin tertiary structure. 
Figure 4.9 - Effects of Chlorin e6 (Ce6) and $\mathrm{Zn}^{2+}$ on thermal stability of rhodopsin at $55{ }^{\circ} \mathrm{C}$ using fluorescence spectroscopy.

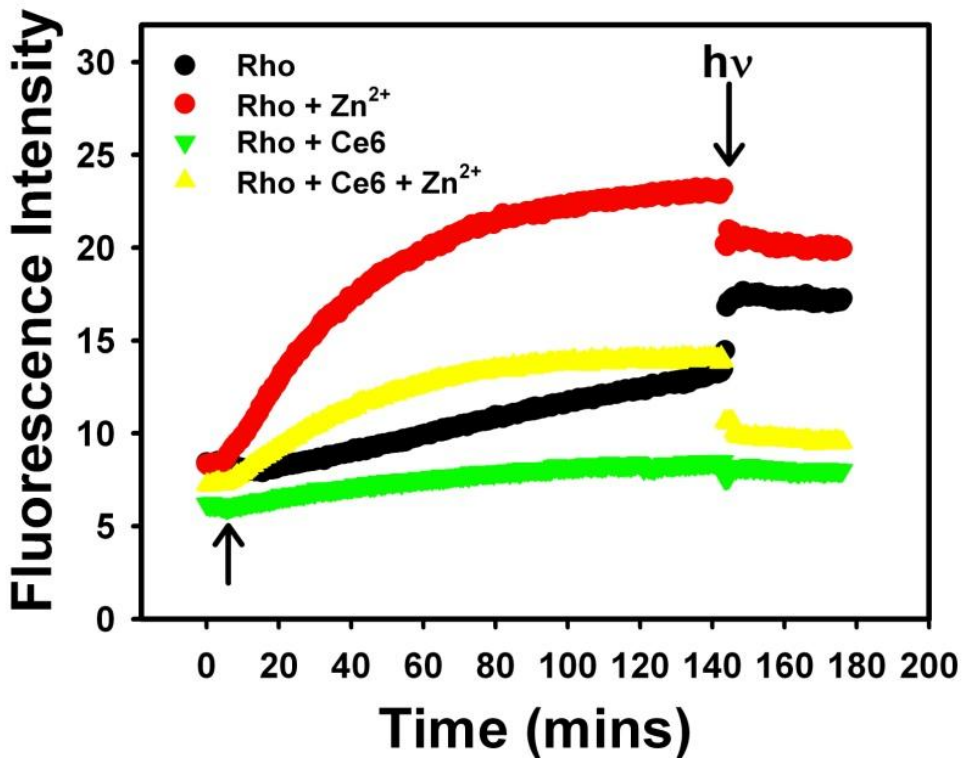

Concentrated used were: Rhodopsin $0.5 \mu \mathrm{M}$, Ce6 $1.25 \mu \mathrm{M}$ and $\mathrm{Zn}^{2+} 100 \mu \mathrm{M}$. An excitation slit-width of $5 \mathrm{~nm}$ and emission slit-width of $5 \mathrm{~nm}$ was used to record the fluorescence traces shown here. Samples were first recorded at $20{ }^{\circ} \mathrm{C}$ for 5 minutes and then the temperature was changed to $55^{\circ} \mathrm{C}$ (indicated by an upward arrow). After 140 minutes the samples were illuminated using a $>495 \mathrm{~nm}$ light source (indicated by a downward arrow).

SOURCE: Balen, 2012

\subsubsection{Effects of Ce6 and bivalent metal ions binding on the Meta II decay of rhodopsin}

It was previously shown that the formation of the light-activated Meta II state of rhodopsin is unaffected by the addition of $\mathrm{Zn}^{2+}$ (Valle et al., 2003). Fluorescence traces of rhodopsin in the presence and absence of $\mathrm{Zn}^{2+}$ are shown in Figure 4.10 (red and black lines, respectively). At 50 -fold excess $(25 \mu \mathrm{M})$ of $\mathrm{Zn}^{2+}$ over rhodopsin $(0.5 \mu \mathrm{M})$, there was no change in the fluorescence in the dark. Upon light-activation, a slight decrease in maximal tryptophan fluorescence was observed in the presence of $\mathrm{Zn}^{2+}$ and the rate of formation of maximal fluorescence after light-activation was also decreased. This indicates that global properties of opsin structure and the stability of the Meta II state in the presence of $\mathrm{Zn}^{2+}$ may have been altered.

Previously, it was found that $\mathrm{Cu}^{2+}$ is able to displace $\mathrm{Zn}^{2+}$ from rhodopsin (Shuster et al., 1992) and induces similar destabilizing effects on the dark-state chromophore as $\mathrm{Zn}^{2+}$ (Shuster et al., 1992; Valle et al., 2003). Therefore it was tested if other metal ions in addition to $\mathrm{Zn}^{2+}$ would also alter tryptophan fluorescence after light activation. The results obtained with $\mathrm{Fe}^{2+}, \mathrm{Cu}^{2+}$, 
$\mathrm{Ni}^{2+}$ and $\mathrm{Mg}^{2+}$ are shown in comparison to $\mathrm{Zn}^{2+}$ and rhodopsin alone in Figure 4.10. Upon illumination, metal ions exhibited differential effects on the rhodopsin maximal fluorescence signal. A decrease in tryptophan fluorescence similar to that observed in the presence of $\mathrm{Zn}^{2+}$ was seen with $\mathrm{Fe}^{2+}$ and $\mathrm{Cu}^{2+}$, with $\mathrm{Cu}^{2+}$ displaying the largest change amongst all metal ions tested. In contrast, $\mathrm{Ni}^{2+}$ and $\mathrm{Mg}^{2+}$ showed a nominal increase in fluorescence as compared to rhodopsin alone. The increase in fluorescence upon light activation was fitted to a non-linear regression function to calculate the decay rates and half-life values in each case. The results are shown in Table 4.3. The stability of the Meta II state in the presence of $\mathrm{Fe}^{2+}$, like $\mathrm{Ni}^{2+}$, was similar to Meta II alone. Rhodopsin in the presence of $\mathrm{Zn}^{2+}$ and $\mathrm{Mg}^{2+}$ exhibited increased Meta II half-lives. $\mathrm{Cu}^{2+}$ was the only metal ion that greatly affected the stability of the Meta II state. The decay rate increased approximately by a factor of 2 . It was not possible to fit the data reliably in the presence of $\mathrm{Cu}^{2+}$ because of the competing decrease in fluorescence at later times in the presence of this metal ion. 
Figure 4.10 - Effect of metal ions on rhodopsin Meta II decay monitored using fluorescence spectroscopy.

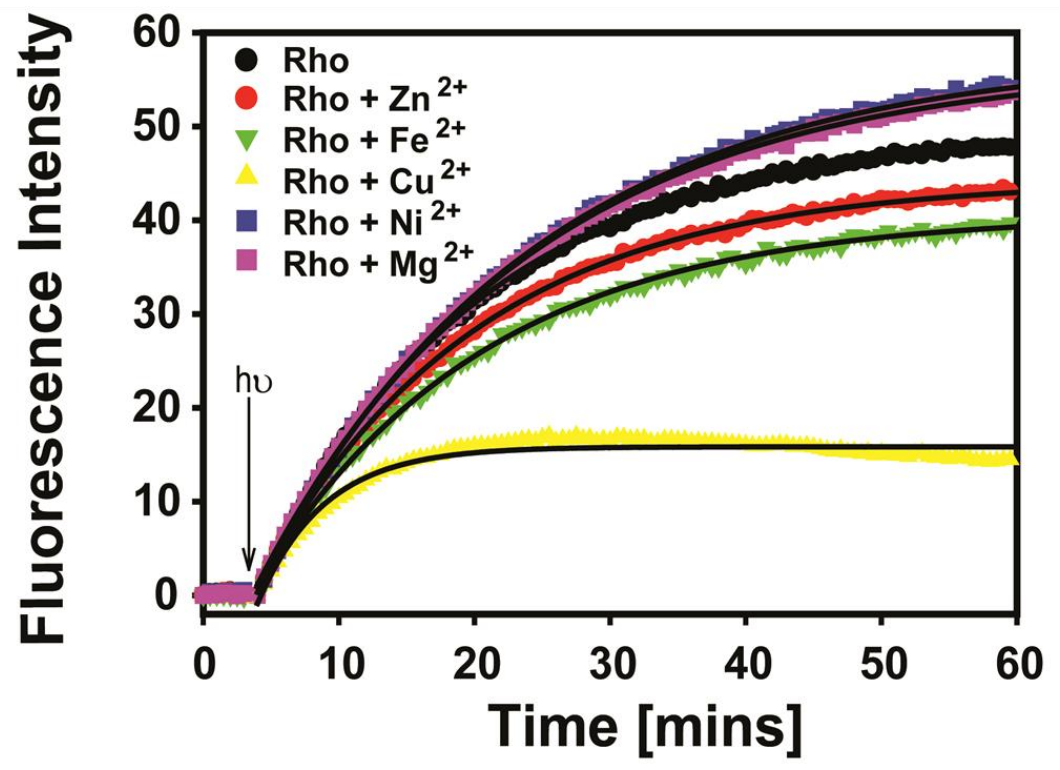

The illumination point is indicated by an arrow. Fluorescence spectra of rhodopsin Meta II decay in the absence (black circles) and presence of $\mathrm{Zn}^{2+}$ (red circles), $\mathrm{Fe}^{2+}$ (green triangles), $\mathrm{Cu}^{2+}$ (yellow triangles), $\mathrm{Ni}^{2+}$ (blue squares), and $\mathrm{Mg}^{2+}$ (purple squares). Excitation and emission wavelengths of $280 \mathrm{~nm}$ and $330 \mathrm{~nm}$ and slit widths of $5 \mathrm{~nm}$ and $10 \mathrm{~nm}$ were used. The data was fitted using the exponential rise to maximum function in Sigmaplot 10.0.

SOURCE: Figure is adapted from (Balen, 2009). With permission.

Table 4.3 - The half-lives and Meta II decay rates of rhodopsin in the absence and presence of metal ions.

\begin{tabular}{ccc}
\hline & $\mathrm{t}_{1 / 2}(\mathrm{~min})$ & $\begin{array}{c}\text { Decay rate } \\
\left(\mathrm{min}^{-1}\right)\end{array}$ \\
\hline Rho & $13.7 \pm 0.2$ & $0.051 \pm 0.001$ \\
$\mathrm{Rho}+\mathrm{Zn}^{2+}$ & $15.5 \pm 2.5$ & $0.046 \pm 0.008$ \\
$\mathrm{Rho}+\mathrm{Fe}^{2+}$ & $13.0 \pm 0.2$ & $0.053 \pm 0.001$ \\
$\mathrm{Rho}+\mathrm{Cu}^{2+}$ & $5.4 \pm 1.4$ & $0.134 \pm 0.036$ \\
$\mathrm{Rho}+\mathrm{Ni}^{2+}$ & $14.7 \pm 1.3$ & $0.047 \pm 0.004$ \\
& & \\
$\mathrm{Rho}+\mathrm{Mg}^{2+}$ & $15.2 \pm 0.4$ & $0.046 \pm 0.001$ \\
\hline
\end{tabular}

SOURCE: Balen, 2012 


\subsubsection{Cyanidin-3-Glucoside (C3G) interaction with rhodopsin}

The term "anthocyanin" refers to a broad spectrum of compounds with many derived species. These derivatives do not only present a variation in color, which is due to differences in the chemical structures but also due to changes in $\mathrm{pH}$, solvent and presence or absence of copigments (Brouillard and Dubois, 1977; Malien-Aubert et al., 2001; Veitch and Grayer, 2008). Previous studies have characterized $\mathrm{C} 3 \mathrm{G}$ species at different $\mathrm{pH}$ values. These studies are described in detail in the $\mathrm{PhD}$ theses of Naveena Yanamala (Yanamala, 2009) and of Kalyan Tirupula (Tirupula, 2011) and are published in (Tirupula et al., 2009; Yanamala et al., 2009). This chapter shows the molecular effects of $\mathrm{C} 3 \mathrm{G}$ binding to rhodopsin at different $\mathrm{pH}$ values, via stability studies using CD, fluorescence and absorbance spectroscopy.

\subsubsection{Effects of C3G binding on secondary structure of rhodopsin}

Binding of ligands often enhances the thermal stability of the proteins they are binding to (Vedadi et al., 2006). We therefore tested if $\mathrm{C} 3 \mathrm{G}$ affects the thermal stability of rhodopsin. Figure 4.11 shows the effect of $\mathrm{C} 3 \mathrm{G}$ on the thermal stability of secondary structure of the rhodopsin measured using circular dichroism (CD) spectroscopy. The MRE was followed at 222 $\mathrm{nm}$ for $1.5 \mu \mathrm{M}$ rhodopsin at $\mathrm{pH} 4, \mathrm{pH} 6$ and $\mathrm{pH} 8$ with temperature increasing from $5{ }^{\circ} \mathrm{C}$ to 100 ${ }^{\circ} \mathrm{C}$. In the absence of $\mathrm{C} 3 \mathrm{G}$ (Figure 4.11A), the overall decrease in MRE as a function of temperature is indistinguishable for $\mathrm{pH} 4$ and $\mathrm{pH} \mathrm{8}$, and both are slightly higher as compared to rhodopsin at $\mathrm{pH}$ 6. The mid-point transition temperatures decrease with increasing $\mathrm{pH}: 69 \pm 4{ }^{\circ} \mathrm{C}$ at $\mathrm{pH} 4,64 \pm 5{ }^{\circ} \mathrm{C} \mathrm{pH} 6$ and $57 \pm 2{ }^{\circ} \mathrm{C}$ at $\mathrm{pH}$ 8. The results were quite different when $\mathrm{C} 3 \mathrm{G}$ was present. Overall, there was a small but significant and reproducible decrease in helicity in the presence of a 10 -fold excess $(15 \mu \mathrm{M}) \mathrm{C} 3 \mathrm{G}$ over rhodopsin $(1.5 \mu \mathrm{M})$, the extent of decrease in MRE at $\mathrm{pH} 4$ and $\mathrm{pH} 6$ being higher as compared to $\mathrm{pH} 8$ (Figure 4.11B). The normalized overall difference between MRE at $5{ }^{\circ} \mathrm{C}$ and $100{ }^{\circ} \mathrm{C}$ was the same in the absence and presence of $\mathrm{C} 3 \mathrm{G}$. The mid-point transition temperature values in the presence of $\mathrm{C} 3 \mathrm{G}$ at $\mathrm{pH} 4, \mathrm{pH} 6$ and $\mathrm{pH}$ 8 are $73 \pm 5{ }^{\circ} \mathrm{C}, 65 \pm 3{ }^{\circ} \mathrm{C}$, and $55 \pm 4{ }^{\circ} \mathrm{C}$, respectively. These studies indicate that $\mathrm{pH}$ affects the stability of secondary structure in rhodopsin, with a significant decrease in mid-point transition temperatures towards higher $\mathrm{pH}$ values. While $\mathrm{C} 3 \mathrm{G}$ did not alter this trend, its presence did have 
small but significant effects on the shapes of the thermal melting curves at the different $\mathrm{pH}$ values.

Figure 4.11 - The mean residue ellipticity at $222 \mathrm{~nm}$ of rhodopsin $(1.5 \mu \mathrm{M})$ at $\mathrm{pH} \mathrm{4,6}$ and 8 as a function of temperature in the (A) absence and (B) presence of a 10-fold excess of C3G $(15 \mu \mathrm{M})$ over rhodopsin.

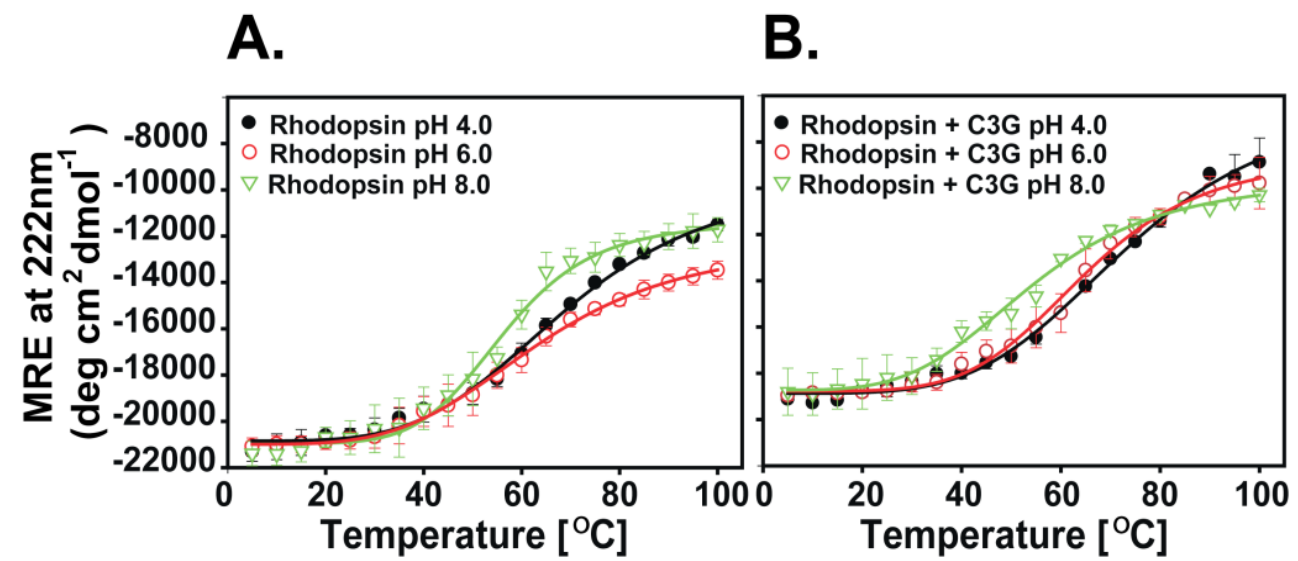

All the samples contained a final DM concentration of $0.05 \%$. The results obtained at $\mathrm{pH} 4,6$ and 8 are represented as red open circles, black solid circles and green triangles, respectively.

SOURCE: Figure is adapted from (Tirupula, 2009). With permission.

\subsubsection{Effects of C3G binding on tertiary structure of rhodopsin}

To test for putative effects of $\mathrm{C} 3 \mathrm{G}$ on tertiary structure of rhodopsin it was investigated whether $\mathrm{C} 3 \mathrm{G}$ binding quenches rhodopsin fluorescence. Figure 4.12 shows the fluorescence traces of rhodopsin in the absence and presence of different concentrations of C3G. There was a concentration-dependent quenching of fluorescence of rhodopsin in both dark and light-activated samples. There was no signal when the ratio of $\mathrm{C} 3 \mathrm{G}$ over rhodopsin was 750 -fold excess, similar to $\mathrm{C} 3 \mathrm{G}$ alone (background signal), suggesting that $\mathrm{C} 3 \mathrm{G}$ binds to both dark and light-activated states of rhodopsin. The data was fitted using a non-linear regression function to estimate binding affinity of interaction. The affinity of interaction of $\mathrm{C} 3 \mathrm{G}$ with rhodopsin was calculated as $90 \mu \mathrm{M}$ for the Meta II state and $450 \mu \mathrm{M}$ for the dark state. 
Figure 4.12 - Binding of C3G to rhodopsin in the dark and in the light as monitored by fluorescence spectroscopy.

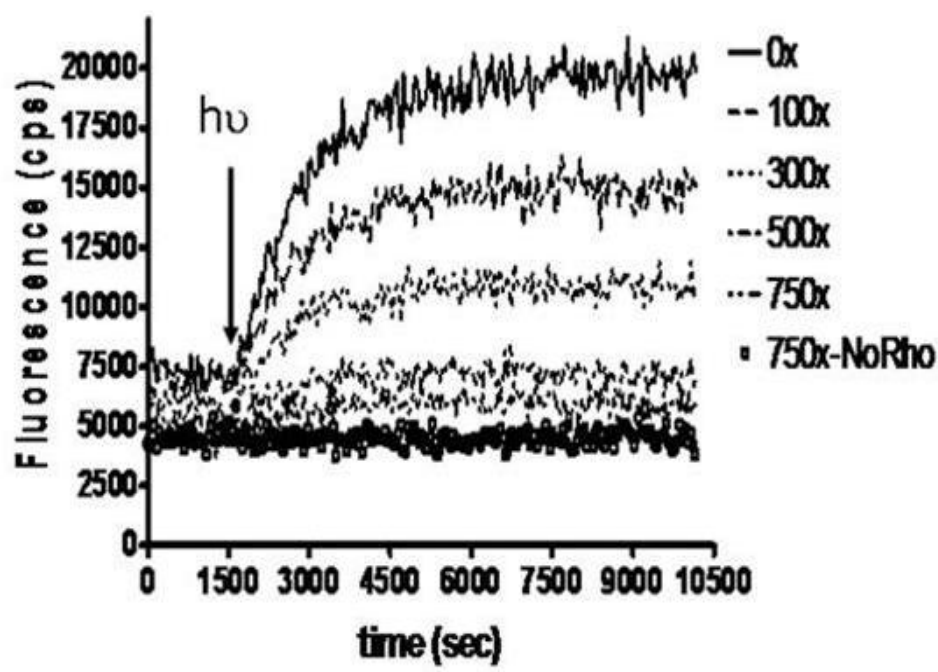

Fluorescence traces obtained at wavelengths for excitation of $280 \mathrm{~nm}$ and for emission of $330 \mathrm{~nm}$ in the presence of increasing amounts of $\mathrm{C} 3 \mathrm{G}$.

SOURCE: Image courtesy of Naveena Yanamala (Yanamala, 2009).

\subsubsection{3 $\mathrm{pH}$ dependent alteration of structure and stability of rhodopsin in the absence and presence of $C 3 G$}

Thermal stability of rhodopsin tertiary structure investigated by fluorescence spectroscopy is shown in Figure 4.13 and 4.14. Figure 4.13 shows the thermal denaturation of rhodopsin alone and in the presence of $\mathrm{C} 3 \mathrm{G}$ at $\mathrm{pH} 4,6$ and 8 . In the absence of $\mathrm{C} 3 \mathrm{G}$, an overall increase in rhodopsin fluorescence is observed at $\mathrm{pH} 4$ and 8 as compared to that of $\mathrm{pH}$. At $\mathrm{pH}$ 6 no difference in the rhodopsin fluorescence traces in the absence and presence of $\mathrm{C} 3 \mathrm{G}$ was observed. Although there was no change in the overall profile of the fluorescence traces at $\mathrm{pH} 4$ and 8, a decrease in fluorescence intensity was noted. The major transition in all cases appears to be complete at $60{ }^{\circ} \mathrm{C}$, after which the fluorescence decreases, presumably due to aggregation. 
Figure 4.13 - Fluorescence traces of thermal denaturation of rhodopsin in the absence (black lines) and presence (red lines) of $\mathrm{C3G}$ at (A) $\mathrm{pH} \mathrm{4,} \mathrm{(B)} \mathrm{pH} 6$ and (C) pH 8.
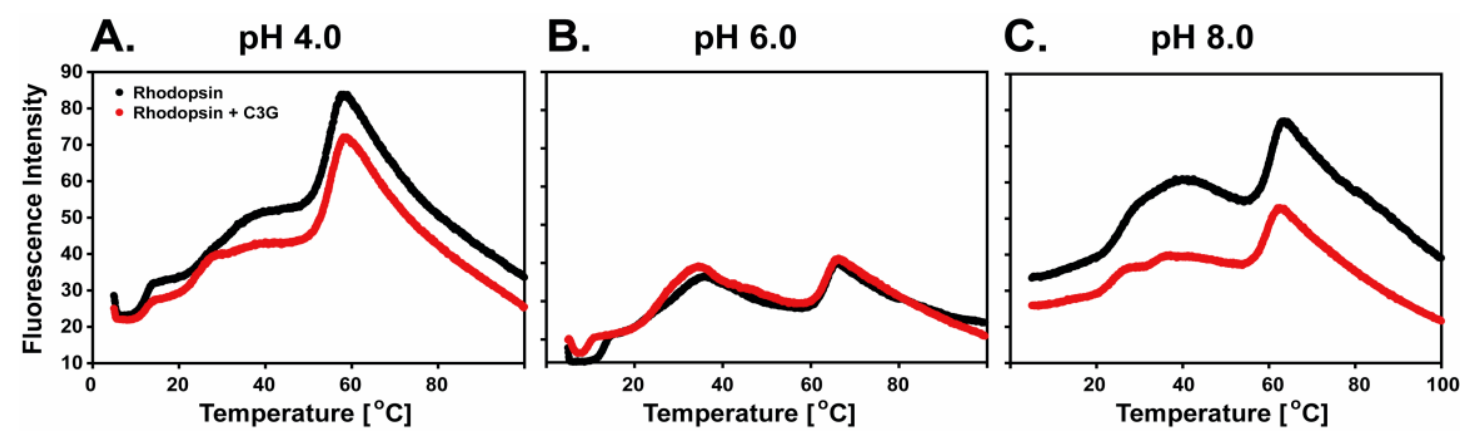

Temperature was increased from $5{ }^{\circ} \mathrm{C}$ to $100{ }^{\circ} \mathrm{C}$. The total time of the experiment is approximately 4 hours. Because the absorbance of $\mathrm{C} 3 \mathrm{G}$ changes with $\mathrm{pH}$, the actual fluorescence counts in the presence and absence of $\mathrm{C} 3 \mathrm{G}$ are slightly different for different $\mathrm{pH}$ values. Correction for these intensity effects in the fluorescence traces shown were not done, as the primary interest was the shape of the curves. Excitation and emission wavelengths of $280 \mathrm{~nm}$ and $330 \mathrm{~nm}$ and slit widths of $5 \mathrm{~nm}$ and $10 \mathrm{~nm}$ were used.

SOURCE: Figure is adapted from (Tirupula, 2009). With permission.

Figure 4.14 displays the effects of $\mathrm{C} 3 \mathrm{G}$ on changes in tryptophan fluorescence at elevated temperatures, $55{ }^{\circ} \mathrm{C}$, over time. Here, the thermal stability of the retinal-protein interactions is measured, without illuminating the samples. Therefore, especially the time dependence of fluorescence changes at $55{ }^{\circ} \mathrm{C}$, and the mid-point of the transition just before reaching maximal fluorescence, was investigated in more detail.

Samples were first kept at $20{ }^{\circ} \mathrm{C}$ for 5 minutes, after which the temperature was raised to $55{ }^{\circ} \mathrm{C}$ and the increase in fluorescence was monitored over time. The fluorescence traces of rhodopsin in the absence and presence of $\mathrm{C} 3 \mathrm{G}$ at $\mathrm{pH} 4$ are shown in Figure 4.14A, 6 in Figure 4.14B and 8 in Figure 4.14C. Maximal fluorescence of rhodopsin in the absence of C3G was always higher (black lines) as compared to rhodopsin in the presence of $\mathrm{C} 3 \mathrm{G}$ (grey lines) due to the light intensity decrease as a result of $\mathrm{C} 3 \mathrm{G}$ absorption. Because the major results of this experiment are the rates at which maximal fluorescence was reached, the decrease in fluorescence in the traces shown in Figure 4.14 was not corrected. At pH 6 (Figure 4.15B), the thermal decay rate of rhodopsin in the presence of $\mathrm{C} 3 \mathrm{G}$ was accelerated approximately 2-fold as compared to rhodopsin alone. The $t_{1 / 2}$ values in the presence and absence of $\mathrm{C} 3 \mathrm{G}$ were $73 \pm 6$ minutes and $111 \pm 11$ minutes, respectively. At $\mathrm{pH} 4$ and $\mathrm{pH} 8$, the stability of rhodopsin tertiary structure was greatly decreased as compared to $\mathrm{pH}$ 6. At $\mathrm{pH} 4$ (Figure 4.14A), a $t_{1 / 2}$ value of 
4.9 \pm 0.9 minutes and at $\mathrm{pH} 8$ (Figure $4.14 \mathrm{C}$ ) a $t_{1 / 2}$ value of $15.7 \pm 0.7$ minutes was observed. In the presence of $\mathrm{C} 3 \mathrm{G}$, the corresponding $t_{1 / 2}$ values were $4.5 \pm 0.1$ minutes and $13.5 \pm 2.2$ minutes, respectively. These results are in agreement to those described above for regeneration: rhodopsin is destabilized at extremely low and high $\mathrm{pH}$ values and $\mathrm{C} 3 \mathrm{G}$ has negligible effects on stability under these conditions. In contrast, at $\mathrm{pH} 6$, where stability is highest, $\mathrm{C} 3 \mathrm{G}$ does show a destabilizing effect on rhodopsin tertiary structure.

Figure 4.14 - Stability of rhodopsin $(0.5 \mu \mathrm{M}$ ) in the absence (black lines) and presence of $5 \mu \mathrm{M}$ C3G (gray lines) at $55^{\circ} \mathrm{C}$ measured by fluorescence spectroscopy at (A) pH 4, (B) $\mathrm{pH} 6$ and (C) $\mathrm{pH} 8$.

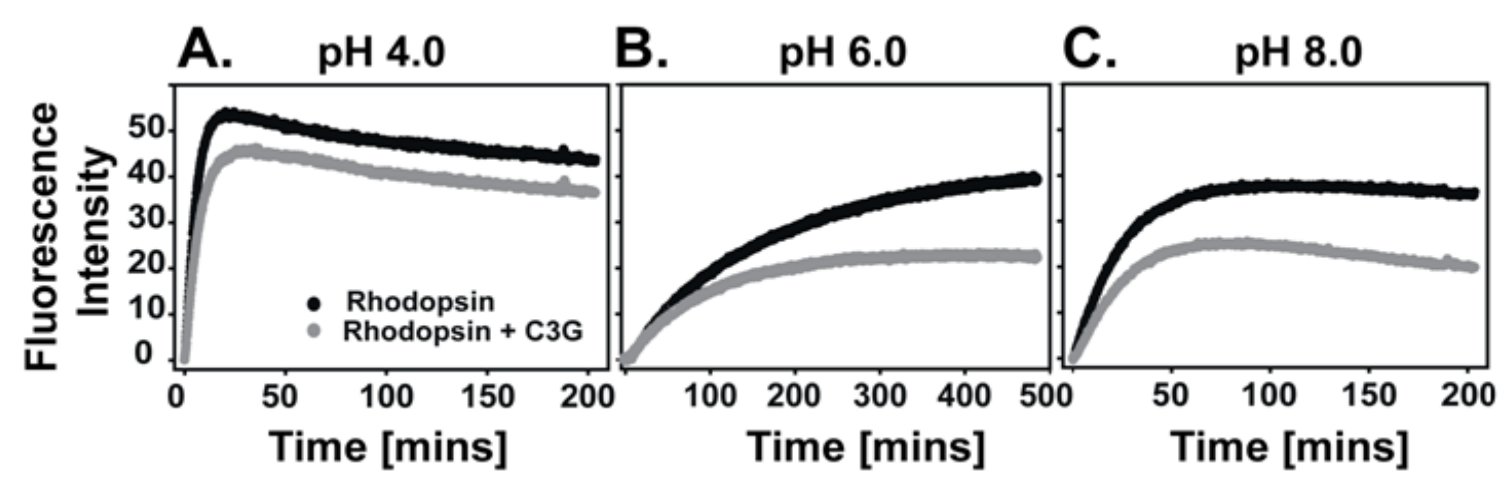

A total DM concentration of $0.05 \%$ was used in all experiments. The experiment was monitored for 200 minutes at $\mathrm{pH} 4$ and 8 and for 500 minutes at pH 6. Excitation and emission wavelengths of $280 \mathrm{~nm}$ and $330 \mathrm{~nm}$ and slit widths of $5 \mathrm{~nm}$ and $10 \mathrm{~nm}$ were used.

SOURCE: Figure is adapted from (Tirupula, 2009). With permission.

In Figure 4.15, the thermal decay of chromophore-protein interactions at $50{ }^{\circ} \mathrm{C}$ as measured by UV/Visible absorbance spectroscopy are shown. The loss of $500 \mathrm{~nm}$ chromophore and the increase of the $380 \mathrm{~nm}$ free retinal peak were followed over time. Interestingly, there was a certain tendency to sigmoidal shape in the decay curves that is not as apparent at $\mathrm{pH} 6$ in the absence of $\mathrm{C} 3 \mathrm{G}$. Nevertheless, the data was analyzed using exponential decay functions for consistency with previous analysis of thermal decay data (Stojanovic et al., 2004; Valle et al., 2003). At $\mathrm{pH} 4$ (Figure 4.15A) a decay rate of $0.077 \pm 0.004$ minutes $^{-1}$ and $0.110 \pm 0.004$ minutes $^{-1}$ was observed for rhodopsin in the absence and presence of $\mathrm{C} 3 \mathrm{G}$ respectively, with $t_{1 / 2}$ values of $9.0 \pm 0.4$ minutes and $6.3 \pm 0.3$ minutes respectively, showing a decrease in stability in the presence of $\mathrm{C} 3 \mathrm{G}$ at this $\mathrm{pH}$. The effect is even more pronounced at the higher $\mathrm{pH}$ values, $\mathrm{pH} 6$ (Figure 4.15B) and $\mathrm{pH} 8$ (Figure 4.15C). The rates of decay at $\mathrm{pH} 6$ were accelerated approximately 3 - 
fold in the presence of $\mathrm{C} 3 \mathrm{G}$, with observed rates of $0.025 \pm 0.003$ minutes $^{-1}$ and $0.066 \pm 0.001$ minutes $^{-1}$, corresponding to a $t_{1 / 2}$ of $27.7 \pm 1.7$ minutes and $10.5 \pm 0.3$ minutes for rhodopsin without and with $\mathrm{C} 3 \mathrm{G}$, respectively. At $\mathrm{pH} 8$, the presence of $\mathrm{C} 3 \mathrm{G}$ accelerated the decay rate 6fold. The corresponding decay rates in the absence and presence of $\mathrm{C} 3 \mathrm{G}$ were $0.034 \pm 0.003$ minutes $^{-1}$ and $0.205 \pm 0.039$ minutes $^{-1}$ with $t_{1 / 2}$ of $20.3 \pm 1.5$ minutes and $3.4 \pm 0.7$ minutes, respectively. These results show that $\mathrm{C} 3 \mathrm{G}$ destabilizes rhodopsin-retinal interactions in the dark at $50{ }^{\circ} \mathrm{C}$, and the effect is more pronounced at higher $\mathrm{pH}$ values.

Figure 4.15 - Effects of $\mathrm{C} 3 \mathrm{G}$ binding on chromophore stability at $50{ }^{\circ} \mathrm{C}$ monitored by UV/Visible Spectroscopy, (A) at $\mathrm{pH} \mathrm{4,} \mathrm{(B)} \mathrm{at} \mathrm{pH} \mathrm{6,} \mathrm{and} \mathrm{(C)} \mathrm{at} \mathrm{pH} 8$.
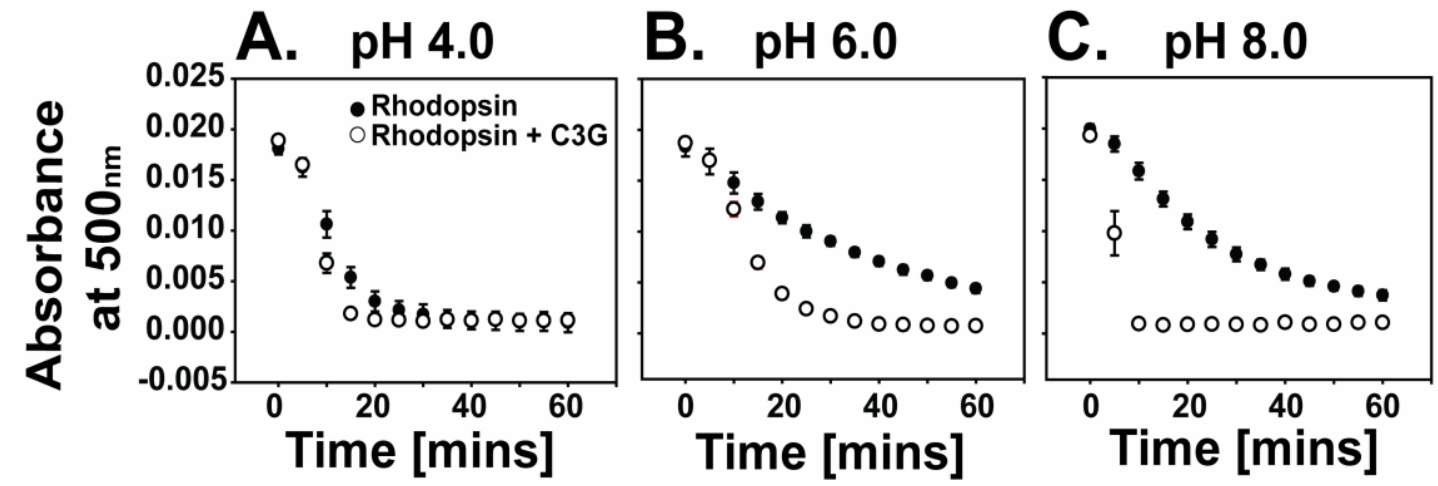

Rhodopsin $(0.5 \mu \mathrm{M})$ in the absence and presence of $\mathrm{C} 3 \mathrm{G}(0.5 \mu \mathrm{M})$ is shown using solid and open circles, respectively.

SOURCE: Figure is adapted from (Tirupula, 2009). With permission.

\subsection{DISCUSSION}

The goal of this study was to investigate whether the different small molecules that have been implicated as putative allosteric ligands of rhodopsin have direct effects on rhodopsin structure and stability. In a systematic approach, the thermal stability of rhodopsin secondary and tertiary structure in vitro was repeated, in the absence or presence of other molecules, using classical biophysical approaches, namely CD, UV/Visible and fluorescence spectroscopy. 


\subsection{1 $\mathrm{Zn}^{2+}$ and other metal effects}

The first molecule studied was $\mathrm{Zn}^{2+}$. The deficiency of $\mathrm{Zn}^{2+}$ is detrimental to retinal function, however $\mathrm{Zn}^{2+}$ supplementation has yielded contradictory effects in patients affected with age-related macular degeneration (AMD) disease (Lengyel and Peto, 2008; Lengyel and Peto, 2008). Thus, it is critical to investigate the molecular actions of $\mathrm{Zn}^{2+}$. Previous studies have established the visual photoreceptor rhodopsin as a target for $\mathrm{Zn}^{2+}$ and a modulation of rhodopsin stability has emerged as its predominant effect (Stojanovic et al., 2004; Valle et al., 2003). However, there has been discrepancy on whether these effects are stabilizing or destabilizing (Park et al., 2007; Stojanovic et al., 2004; Valle et al., 2003). Binding of ligands is known to stabilize their protein partners (Bullock et al., 2005; Vedadi et al., 2006) and the discrepancy is therefore surprising. Therefore, a detailed investigation of the effects of bivalent metal ions on stability of rhodopsin was done here. Using Circular Dichroism, UV/Vis- and fluorescence spectroscopy it was shown that $\mathrm{Zn}^{2+}$ exerts a dual effect on rhodopsin thermal stability in vitro. On the one hand, fluorescence spectroscopy suggests that $\mathrm{Zn}^{2+}$ loosens tertiary structure, providing a mechanism for retinal to leave more easily as seen by the faster thermal decay of the retinal chromophore. This, for the first time, provides a plausible molecular mechanism for the previously observed increases in rates of chromophore absorbance loss (Stojanovic et al., 2004; Valle et al., 2003). On the other hand, $\mathrm{Zn}^{2+}$ stabilizes secondary structure, indicating a global stabilization of protein structure. The studies represent the first direct measurements of thermal protein stability by analysis of secondary structure, a bulk biophysical technique. The findings support the conclusions reached by single molecule AFM spectroscopy measurements (Park et al., 2007) and show that previous "destabilizing" effects (Stojanovic et al., 2004; Valle et al., 2003) are not destabilizing the rhodopsin structure as a whole but rather destabilize retinalprotein interactions alone.

Other divalent metal ions $\mathrm{Cu}^{2+}, \mathrm{Fe}^{2+}, \mathrm{Ni}^{2+}, \mathrm{Mg}^{2+}$ and $\mathrm{Mn}^{2+}$ were also investigated. Each of these exhibits some effects on rhodopsin thermal stability. The most dramatic effects were observed with $\mathrm{Zn}^{2+}, \mathrm{Cu}^{2+}$ and $\mathrm{Fe}^{2+}$. These results are consistent with previous studies where it was shown that $\mathrm{Cu}^{2+}$ was most similar to $\mathrm{Zn}^{2+}$ in the extent of destabilization of retinalchromophore interactions (Valle et al., 2003). $\mathrm{Cu}^{2+}$ was also shown to compete best with $\mathrm{Zn}^{2+}$ in binding to rhodopsin (Shuster et al., 1992), among a set of metal ions tested including $\mathrm{Cd}^{2+}, \mathrm{Ca}^{2+}$, 
$\mathrm{Co}^{2+}, \mathrm{Mg}^{2+}$ and $\mathrm{Mn}^{2+}$ (Shuster et al., 1992). Furthermore, it was found that $\mathrm{Cu}^{2+}$ alone significantly alters the stability of Meta II and probably leads to its aggregation indicated by the decrease in tryptophan fluorescence observed in the presence of $\mathrm{Cu}^{2+}$ at prolonged incubation periods.

\subsubsection{Ce6 effects with and without bivalent metal ions}

In addition to binding of $\mathrm{Zn}^{2+}$ to rhodopsin, that of other nutrients was also studied, with the following rationale: The effects of nutrients on the visual system are widely recognized, including trace metals, vitamins, and pigments. Often nutrients work additively. For example, both Vitamin $\mathrm{A}$ and $\mathrm{Zn}^{2+}$ supplements are necessary to gain improvements in electroretinograms of rats deprived of $\mathrm{Zn}^{2+}$ and/or vitamin A (Kraft et al., 1987). Therefore it was hypothesized that $\mathrm{Zn}^{2+}$ may show enhanced effects if combined with other nutrients. The molecule that was chosen to test this effect was the chlorophyll-derivative Ce6 because of its known role in visual photosensitization, which has recently been confirmed in mouse models (Washington et al., 2007).

The binding of Ce6 to rhodopsin can be seen by the quenching of rhodopsin's tryptophan fluorescence. Measurements of CD spectra in the presence of Ce6 show a shift in the MRE at $222 \mathrm{~nm}$, indicating a stabilization of its $\alpha$-helix content, even over a wide range of temperatures $\left(5{ }^{\circ} \mathrm{C}\right.$ to $\left.100{ }^{\circ} \mathrm{C}\right)$. The combined effects of $\mathrm{Ce} 6$ and $\mathrm{Zn}^{2+}$ on rhodopsin thermal stability were investigated by monitoring the loss of secondary and tertiary structure as a function of time at constant temperature, as well as over the $5{ }^{\circ} \mathrm{C}$ to $100{ }^{\circ} \mathrm{C}$ temperature range. The results indicate an additive nature of these two compounds. In particular, the degree of $\alpha$-helix content lost in the presence of $\mathrm{Ce} 6$ and $\mathrm{Zn}^{2+}$ as a function of temperature was smaller compared to rhodopsin alone or in the presence of either compound alone. On reversing the thermal cycle $\left(100{ }^{\circ} \mathrm{C}\right.$ to $\left.5{ }^{\circ} \mathrm{C}\right)$, no changes on the total $\alpha$-helical content was observed, as opposed to the further decrease in helicity that was observed for rhodopsin alone. The combined effect was also investigated with all other metal ions, previously described, but the additive effects with Ce6 were most pronounced for $\mathrm{Zn}^{2+}$ and to lesser extent for $\mathrm{Cu}^{2+}$. In contrast, $\mathrm{Fe}^{2+}$ showed similar thermal denaturation curves in the presence and absence of Ce6. 


\subsection{3 $\mathrm{C} 3 \mathrm{G}$}

A third class compounds, anthocyanins, was exemplified by C3G. In general, anthocyanins are known to exert potent antioxidant activity and are very beneficial for human heath (Lila, 2004). More specifically, C3G was found to enhance normal vision, to protect from visual damage and is used as treatment for visual dysfunctions (Lila, 2004; Matsumoto et al., 2001, 2003; Muth et al., 2000; Nakaishi et al., 2000). Parallel studies by PhD students Yanamala and Tirupula aimed to answer the question how anthocyanins aid in regenerating rhodopsin as had been reported by Matsumoto et al. (Matsumoto et al., 2003). They found that the effect of $\mathrm{C} 3 \mathrm{G}$ on regeneration is a direct result of the binding of $\mathrm{C} 3 \mathrm{G}$. Furthermore, $\mathrm{C} 3 \mathrm{G}$ modulates the structure of rhodopsin upon illumination and the presence of $\mathrm{C} 3 \mathrm{G}$ not only changed the rate but also the extent of regeneration (Yanamala, 2009; Yanamala et al., 2009). Studies of Meta II decay rates were performed under the same conditions, but C3G did not significantly change Meta II decay rates (Yanamala, 2009).

Here, we aimed to exploit these findings by checking if these effects on rhodopsin structure result in a stabilization. The stability of secondary and tertiary structures was studied using biophysical methods. Because of the possible $\mathrm{pH}$ dependence, all studies were performed under three different $\mathrm{pH}$ conditions, $\mathrm{pH} 4,6$ and 8. Thermal denaturation over a temperature range of $5{ }^{\circ} \mathrm{C}$ to $100{ }^{\circ} \mathrm{C}$ and at constant elevated temperature $\left(55^{\circ} \mathrm{C}\right)$ showed that, in the presence of $\mathrm{C} 3 \mathrm{G}$, there is a decrease in helix content and thermal decay of tertiary structure is faster as judged from fluorescence spectra. This indicates an overall destabilizing effect of $\mathrm{C} 3 \mathrm{G}$ on rhodopsin structure. When comparing $\mathrm{C} 3 \mathrm{G}$ effects with $\mathrm{pH}$ effects, however, the $\mathrm{pH}$ effects were more prominent as compared to the $\mathrm{C} 3 \mathrm{G}$ effects.

The results from fluorescence spectroscopy point at changes in the stability of tertiary interactions in rhodopsin in the presence of $\mathrm{C} 3 \mathrm{G}$. However, fluorescence spectroscopy measures all tertiary interactions involving tryptophan residues, including protein-protein and proteinligand interactions. To begin to differentiate between these two different types of interactions, the effects of $\mathrm{pH}$ and $\mathrm{C} 3 \mathrm{G}$ on rhodopsin chromophore stability and the rate of retinal leaving from the rhodopsin binding pocket was determined at $50{ }^{\circ} \mathrm{C}$ by $\mathrm{UV} / \mathrm{Visible}$ spectroscopy at the three different $\mathrm{pH}$ values. As previously mentioned, $\mathrm{pH}$ itself significantly altered rhodopsin properties, but here, the $\mathrm{C} 3 \mathrm{G}$ effects were more pronounced as compared to the $\mathrm{pH}$ effects: at 
every $\mathrm{pH}$ value investigated, the decay rate was drastically accelerated. The higher the $\mathrm{pH}$, the more pronounced the $\mathrm{C} 3 \mathrm{G}$ effects became. This clearly demonstrates that the retinal-protein interactions are destabilized in the presence of $\mathrm{C} 3 \mathrm{G}$, especially at higher $\mathrm{pH}$. This indicates that C3G is not a good candidate for further studies of stabilization of RP mutants.

\subsection{CONCLUSIONS}

Three classes of compounds were studied here: (a) divalent metal ions $\mathrm{Zn}^{2+}, \mathrm{Cu}^{2+}, \mathrm{Fe}^{2+}$, $\mathrm{Ni}^{2+}, \mathrm{Mg}^{2+}$ and $\mathrm{Mn}^{2+}$ (b) chlorophyll derivative $\mathrm{Ce}$, and (c) anthocyanin C3G. The interaction of these compounds exerted different effects on the protein rhodopsin.

All divalent metal ions tested presented some effect on the structure of rhodopsin. The $\mathrm{Zn}^{2+}$ effect was the most prominent, but $\mathrm{Cu}^{2+}$ and $\mathrm{Fe}^{2+}$ also displayed a very significant stabilizing effect on rhodopsin structure. Furthermore, not only metal ions, but also Ce6 was able to preserve secondary structure of rhodopsin. In fact, rhodopsin's secondary content was largely preserved by thermal denaturation in the presence of Ce6 even at the highest temperatures tested. This preservation of secondary structure was yet more evident in the presence of both, $\mathrm{Zn}^{2+}$ or $\mathrm{Fe}^{2+}$, the latest being at a lower extent, when combined with $\mathrm{Ce} 6$. In contrast, $\mathrm{C} 3 \mathrm{G}$ showed an inverse effect, destabilizing the secondary and tertiary structure of rhodopsin. This effect was also shown to be potentiated by $\mathrm{pH}$.

The observation that $\mathrm{Zn}^{2+}$ and $\mathrm{Ce} 6$ but not $\mathrm{C} 3 \mathrm{G}$ exert additive and positive effects on rhodopsin stability opens the door to investigation of potentially additive effects of $\mathrm{Zn}^{2+}$ and other nutrient supplements such as algal extracts for vision in vivo. This is what is exploited in the rest of this thesis. The stabilization obtained by $\mathrm{Ce} 6$ and divalent metal ions, can be potentially relevant to treat dysfunctions caused by nutrient deficiencies, but also for inherited diseases. 


\section{CHAPTER 5: DRUG TREATMENT OF LIGHT-DAMAGED SPRAGUE-DAWLE (SD) RATS IN VIVO}

\subsection{SUMMARY}

Having demonstrated the beneficial effects of Ce6 on stability of rhodopsin structure in vitro (Chapter 4), the next aim was to establish if Ce6 addition may have positive effects on retinal degeneration in vivo. First, the effects of Ce6 treatment on SD rats submitted to lightdamage were assessed. As a control experiment, the synthetic antioxidant DMTU was used, since it is known to prevent retinal damage in normal rats that are submitted to light-retinal damage (Organisciak et al., 1992, 2003). The results obtained in this work with DMTU fully reproduce those previously published where the retinas submitted to light degeneration were totally preserved. Treatment with Ce6, in the other hand, appears to have only a minor effect on retinal damage prevention, not being able to show positive effects such as obtained with DMTU in protecting retinal damage induced by light. Although there was an improvement on the retinal layers organization in the presence of $\mathrm{Ce} 6$, this effect was not homogeneous throughout the retina.

\subsection{INTRODUCTION}

Exposure to bright light and inherited retinal degeneration are involved in the progression and severity of the degenerative process that lead to photoreceptor apoptosis in normal or transgenic animals. Previous work has shown that the exposure of rodent eyes to bright light causes photoreceptor cell damage ( Noel and Albrecht, 1971), and may enhance the progression and severity of age related macular degeneration (AMD) and some forms of RP (Cideciyan et al., 1998; Cruickshanks et al., 1993). In both diseases, the apoptotic pathway is the main cause of light-induced cell death (Wenzel et al., 2005) and through light-induced degeneration the apoptotic cell death can be easily studied.

Many studies using animal models investigated the protection of retinal damage by different molecules. These studies described retinal degeneration results obtained using both rat and mouse animals models (Organisciak et al., 1985, 1992; Ranchon et al., 2003; Tanito et al., 
2002a, 2002b, 2007a; Tomita et al., 2005) as a way to explore new treatments for retinal degeneration.

Retinal damage caused by light exposure in rats and mice can be reduced by the use of various types of antioxidants such as ascorbate (Organisciak et al., 1985), dimethyl thiourea (Organisciak et al., 1992), thioredoxin (Tanito et al., 2002a, 2002b), phenyl-N-tert-butylnitrone (PBN) (Ranchon et al., 2003; Tanito et al., 2007a; Tomita et al., 2005) and TEMPOL (Tanito et al., 2007b).

To investigate the susceptibility of transgenic animal models of RP to light-induced retinal degeneration and test if degeneration can be prevented by antioxidants, the synthetic antioxidants dimethylthiourea (DMTU) (Organisciak et al., 1992, 2003) and tauroursodeoxycholic acid (TUDCA) (Fernandez-Sanchez et al., 2011) were tested. Both molecules showed protective effects, preventing retinal degeneration. In contrast, studies with PBN, that did have preventive effects in light-induced degenerated normal rats, showed no protection against light damage in the RP models (Ranchon et al., 2003). Therefore, the protection obtained by the use of different molecules differs according to the model tested.

In this chapter, the putative effect of $\mathrm{Ce} 6$ in protection against the light-induced retinal degeneration of Sprague Dawley rat was investigated. DMTU was used as a positive control.

\subsubsection{Protection of rat retina from light-induced degeneration with different drugs}

\subsubsection{Degeneration protocol}

Different light-induced degeneration protocols described in the literature for rats were tested and adapted.

The first protocol tested was the one that is already in use at our laboratory for studying retinal degeneration in mice. Two rats were dark adapted overnight. On the next day, the pupils of the rats were first dilated for about 10 minutes in the dark, the animals were placed for two hours in a cage that is internally covered with glass, and a 5000 lux lamp placed on top. Rats were then kept in the dark for 2 days and sacrificed. No degeneration was observed in retinas processed for TUNEL. 
A different protocol was then tested, where the time of exposure to light was increased. Instead of 2 hours, rats were now exposed to bright light for 6 and 8 hours following dark periods of 2 and 3 days, respectively. The duration of light-exposure was chosen based on study of different protocols found in the literature (Ranchon et al., 2001; Tanito et al., 2007a; Tomita et al., 2005), which report provoking a different range of photoreceptor cell apoptosis. The choice was also made in order to accommodate the light source that was already used in the previous experiment. A high density of TUNEL-positive cells in the outer nuclear layer was observed in rats degenerated for 8 hours followed by 3 days of dark adaptation. Degeneration was also detected the other group (6 hours degeneration and 2 days dark adaptation), but in a fewer number of cells (Figure 5.1). Given these results, both protocols can be used as a degeneration protocol, and the choice of protocol depends on the desired severity of retinal degeneration. However, it was surprising to see how a small variation in light exposure period results in such drastic differences in the extent of degeneration. 
Figure 5.1 - Apoptotic photoreceptors after light-induced retinal degeneration.
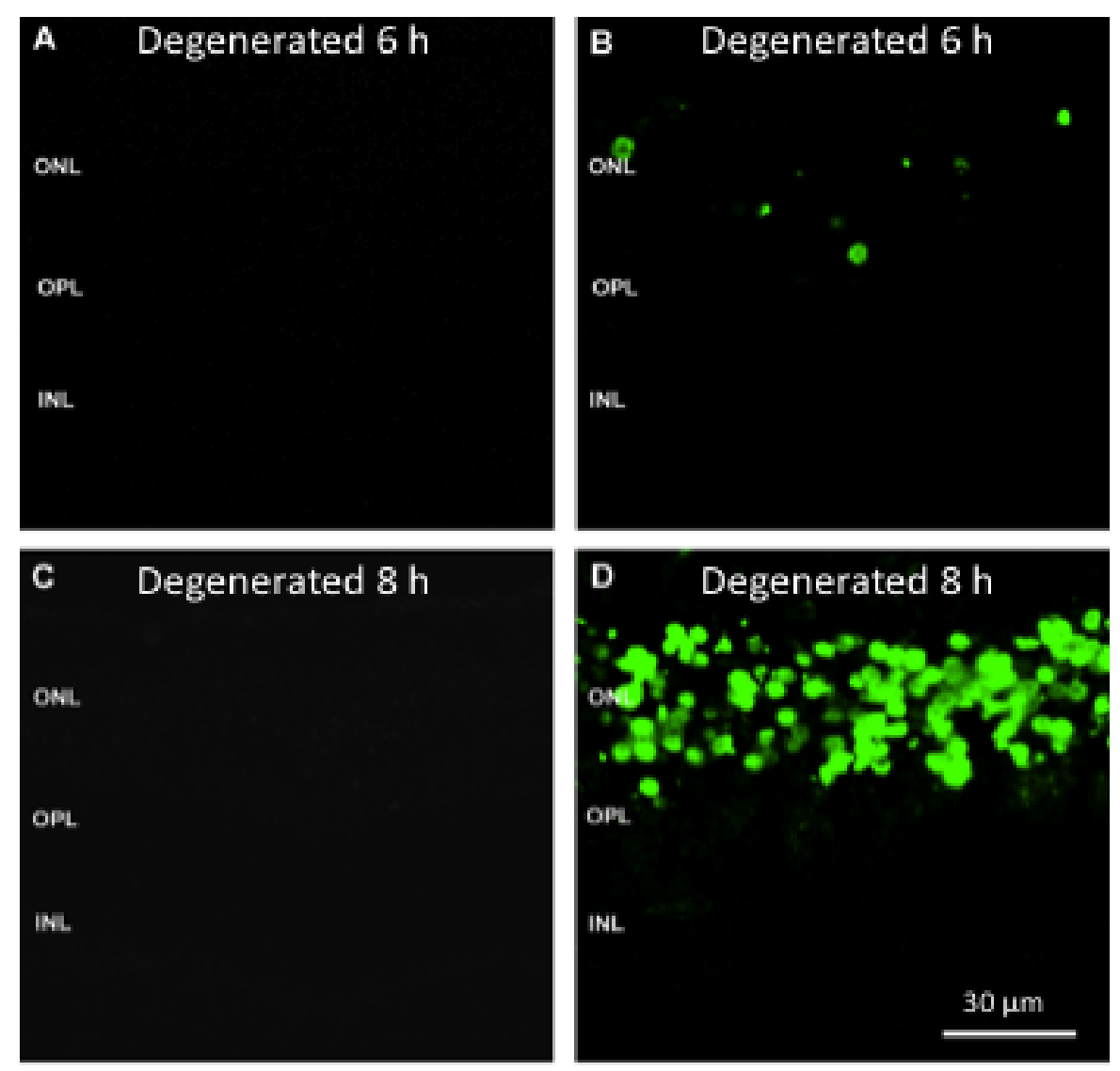

Right column, TUNEL. Left column: control (without the reagent enzyme for TUNEL). Animals were submitted to light-induced degeneration for 6 hours (A and B) or 8 hours (C and D), ONL, outer nuclear layer; OPL, outer plexiform layer; INL, inner plexiform layer. Scale $30 \mu \mathrm{m}$.

SOURCE: Balen, 2012

Because the degree of degeneration appeared highly sensitive to the protocol and was not homogeneous in the whole retinal section among the tested rats, we decided to test a third protocol (Tanito et al., 2007a) that consisted in placing the rats in a dark room for 12 hours, to dilate the pupils, and submit the animals to light-induced retinal degeneration for 6 hours at 5000 lux. After light exposure the rat was placed in a room with normal light/dark cycle (12:12) for 7 days, and then sacrificed. As the degeneration rates can differ depending on day/night time, parameters were kept the same for all experiments. For our studies, this was considered the best protocol, as the results were reproducible among different rats and through the retinas, and was used in the following experiments. Figure 5.2 shows micrographic pictures of retinal sections from a normal rat (left), and a normal but light-degenerated rat (middle and left). 
Figure 5.2 - Comparison of normal and light-induced degeneration, and TUNEL staining.
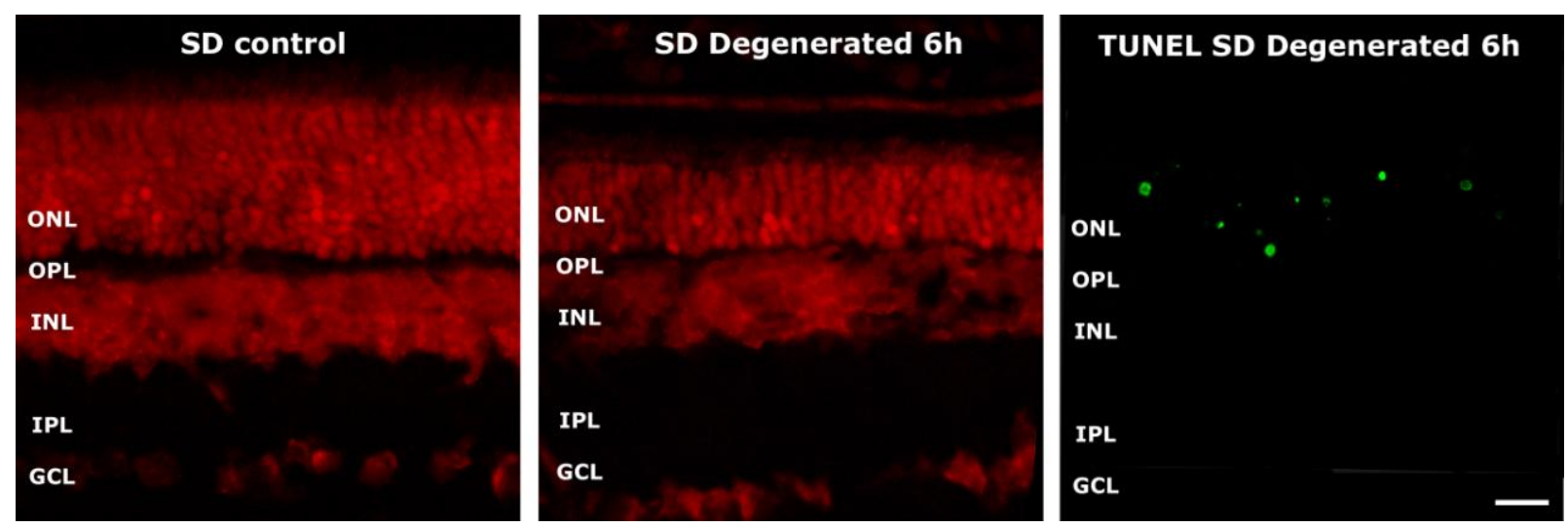

(A) Normal retina (B) light-induced degenerated retina and (C) representative of TUNEL staining of apoptotic cells after degeneration with light. ONL, outer nuclear layer; OPL, outer plexiform layer; INL, inner plexiform layer. Scale $30 \mu \mathrm{M}$.

SOURCE: Balen, 2012

\subsubsection{DMTU effect on light-induced apoptosis of SD rat retina}

To assess the protective effect of DMTU on photoreceptors, intraperitoneal injection of DMTU (500 mg/kg) was administered and rats were subjected to light-degeneration. Retinal degeneration was not homogenous. However, an overall disorganization and marked degeneration pattern could be observed in the rats that were submitted to light-degeneration. In some regions only a few rows of photoreceptors could be observed (Figure 5.3, control).

The thickness and organization of photoreceptor cell layers were very similar for DMTU treated as compared to non-degenerated rats (Figure 5.3). Thus, DMTU treatment was successfully established as a positive control to prevent light-induced retinal degeneration. 
Figure 5.3 - DMTU protects the retina against light-induced degeneration.

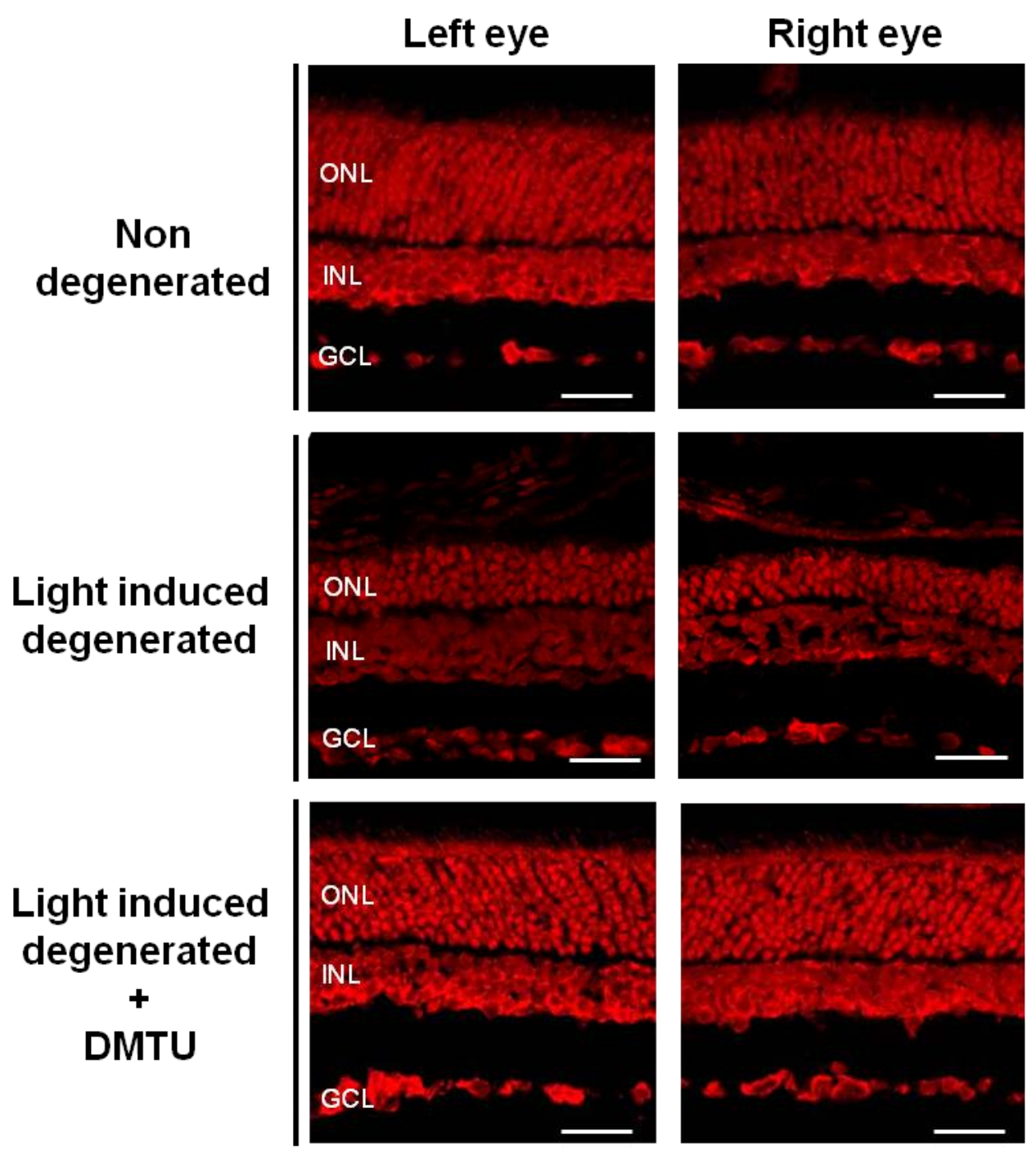

Cross sections of the retina stained with propidium iodide, a nuclear marker. Control rat non-degenerated, lightinduced degenerated rat non-treated and treated with DMTU $(500 \mathrm{mg} / \mathrm{Kg})$. ONL, outer nuclear layer; OPL, outer plexiform layer; INL, inner plexiform layer. Scale $30 \mu \mathrm{M}$.

SOURCE: Balen, 2012

\subsubsection{Ce6 effect on light-induced apoptosis of SD rat retina}

Next, an evaluation whether Ce6 would be able to prevent retinal damage in light exposed animals was performed (Figure 5.4). The protocol was the same as described above, where rats were kept in a normal dark/light cycle. Ce6 was injected for the first time 24 hours before 
degeneration, at different doses, 2, 10 and $20 \mathrm{mg}$, respectively. A second injection was performed $1 / 2$ hour prior to the exposure to light. Following the induction of degeneration, the rat was kept in the normal dark/light cycle for 7 days before being sacrificed.

Differences between treatments could not be fully assessed since the retinal morphology was very similar within different treatments. However, there seems to be a slight improvement in the retinas treated with 2 and $10 \mathrm{mg} / \mathrm{kg}$ where the organization of the photoreceptor layers appears to be more preserved over those treated with $20 \mathrm{mg} / \mathrm{kg}$, which appears to be less and more disordered. The effect was overall less pronounced and less reproducible than that seen with DMTU. 
Figure 5.4 - Effects of Ce6 in light-induced retinal degeneration.

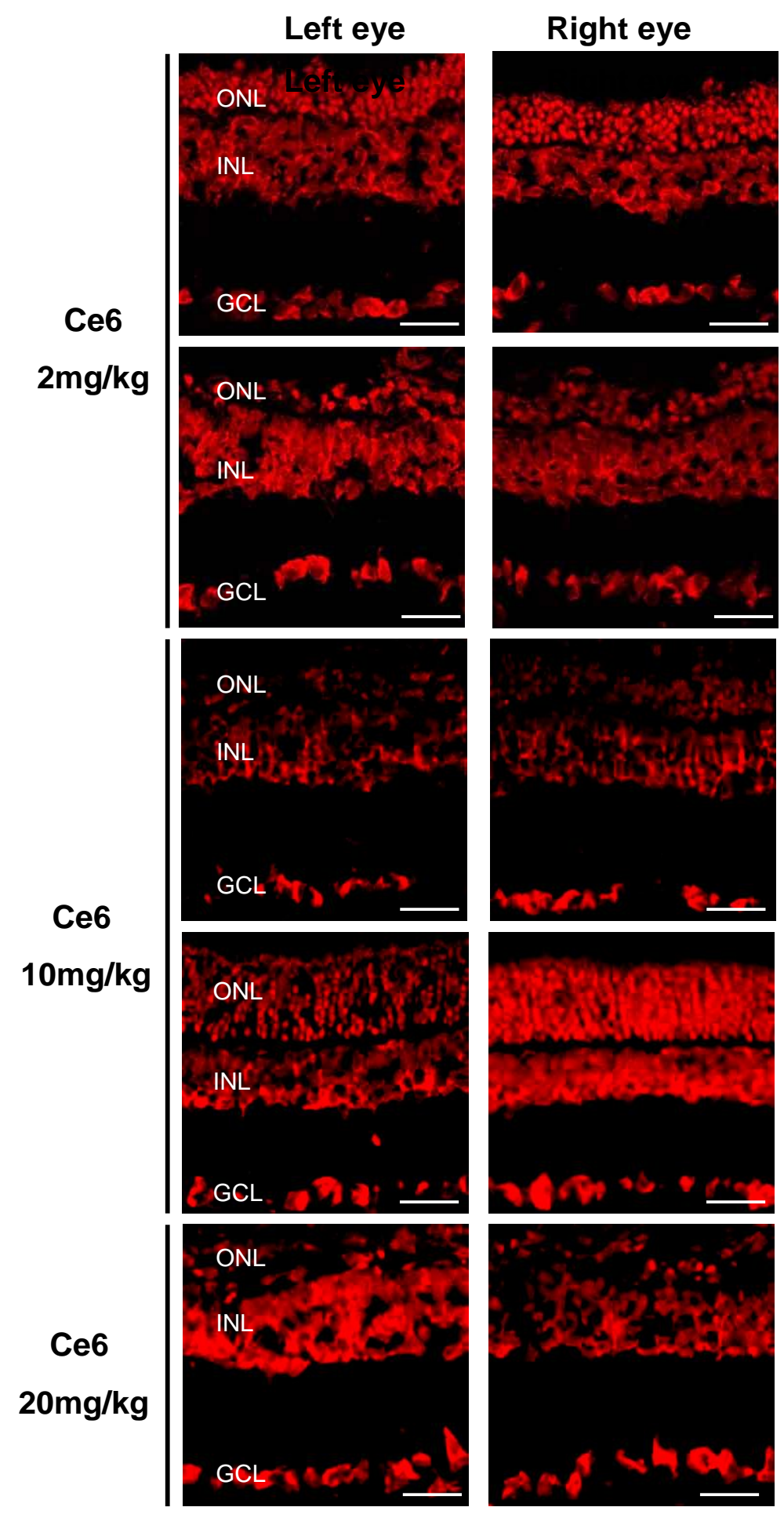

Cross sections of the retina stained with propidium iodide, a nuclear marker. SD rats treated with different concentration of Ce6 $(2(n=2), 10(n=2)$ and $20(n=1) \mathrm{mg} / \mathrm{Kg})$. ONL, outer nuclear layer; INL, inner plexiform layer; GCL, ganglionar cell layer. Scale $30 \mu \mathrm{M}$. 


\subsection{DISCUSSION}

In this chapter, the aim was to investigate whether $\mathrm{Ce} 6$ could prevent light-induced retinal degeneration. Unfortunately, treatment with $\mathrm{Ce} 6$ was not able to mimic the positive effect that DMTU has in protecting retinal damage induced by light. The results obtained with DMTU are in agreement with previous work, showing its protective effect against damage by light (Organisciak et al., 1992, 2003). The rat retinas that were submitted to light damage, but were previously treated with DMTU, showed no signs of degeneration. Retinal thickness measurements and morphology fully mimic that of rats that were not treated with light. In contrast, untreated rats not subjected to light-damage presented a strong degeneration pattern.

Unfortunately, treatment with Ce6 was not able to fully mimic the positive effect that DMTU has in protecting from retinal damage induced by light. The control was positive, i.e. DMTU prevented degeneration in all retinal sectional sections analyzed. In contrast, retinal sections of rats treated with Ce6 presented large differences between individual retinal sections analyzed. Therefore, it was not possible to unambiguously ascertain the extent of degeneration versus retinal protection by $\mathrm{Ce} 6$ in light-induced retinal degeneration. This is a common problem of this model for RP. Comparisons of transgenic with normal albino animals demonstrated that the degeneration is not homogeneous throughout the retina when degeneration is induced by light (Fernandez-Sanchez et al., 2011; Ranchon et al., 2001; Steinberg et al., 1996). The properties of light-damage in the SD rats observed here appears similar to these studies, where different regions were also more and others less affected by light exposure. With this cautionary note, however, a detailed comparison between Ce6 treated rats and non-treated rats indicates that treatment may have had an effect, though minor, on retinal damage prevention. Especially, the extent of organization of the layers is improved in the presence of Ce6. We are therefore cautiously optimistic that $\mathrm{Ce} 6$ protects partially against light-damage, with the disclaimer that Ce6 treated rats presented a better organization only in some of the sections while others were similar to that of non-treated animals. 


\section{CHAPTER 6: CE6 TREATMENT OF P23H AND S334TER MUTANT RATS IN VIVO}

\subsection{SUMMARY}

The effects of Ce6 on RP progression in vivo were evaluated by means of functional (ERG) and morphological (histological labeling) techniques in $\mathrm{P} 23 \mathrm{H}$ and S334ter RP rat models. ERG and retinal tissue analysis indicate that Ce6 exerts a small positive functional effect by slowing the rate of $\mathrm{P} 23 \mathrm{H}$ photoreceptor degeneration in vivo. In contrast, $\mathrm{Ce} 6$ increased the photoreceptor degeneration of the S334ter rat in vivo. These studies support the hypothesis that Ce6 affects RP progression in vivo and that these effects are likely linked to the binding of this molecule to rhodopsin as demonstrated in vitro.

\subsection{INTRODUCTION}

Although several studies have been carried out to characterize and understand the mechanism and evolution of retinal degeneration disease caused by mutation in either $\mathrm{P} 23 \mathrm{H}$ or S334ter animal models of RP or in human RP patients, no effective therapies have been developed to date. Therefore, therapies aimed at avoidance or at least delay of retinal degeneration is highly sought-after.

Since Ce6 has shown a positive effect on the stabilization of rhodopsin in vitro (see Chapter 3 of this thesis), an evaluation of the in vivo effects are pursued here throughout techniques that allow assessing the functional (ERG) and morphological (histology) significance of the treatment of rats with $\mathrm{Ce} 6$.

\subsubsection{Rat models}

$\mathrm{P} 23 \mathrm{H}$ and S334ter mutant rhodopsin transgenic rats are well established in vivo model systems for RP (see Chapter 1). Both rats were kindly provided by Matthew LaVail, PhD (UCSF School of Medicine, Beckman Vision Center). P23H rats carry a single amino acid substitution of a proline to histidine at position 23. S334ter rats have a termination codon at residue 334, which 
results in a $\mathrm{C}$-terminal truncated opsin protein, lacking the last 15 amino acid residues and, thus, all of the phosphorylation sites of the protein are removed. All animals were bred in our laboratory against albino Sprague-Dawley (SD) rats in order to produce P23H and S334ter heterozygous rats. These rats have a single transgene allele in addition to the normal two WT opsin alleles, and mimic the RP condition found in humans, such as disease pathology, time course and electrophysiology response, and are valuable models of human ADRP (Sekirnjak et al., 2011). Although both rat models present similar onset of degeneration, which starts between postnatal day (P) 10 and P15, almost coinciding with the time they open their eyes, P12, P23H and S334ter rats were found to present a different pattern already at P30 (Ranchon et al., 2003). In comparison to the WT, the ONL area of the $\mathrm{P} 23 \mathrm{H}$ rat was reduced to $85 \%$, while S334ter was reduced to $70 \%$. Rhodopsin content was reduced to $61 \%$ and $36 \%$ for $\mathrm{P} 23 \mathrm{H}$ and $\mathrm{S} 334$ ter, respectively. Retinal function differences were also observed, where the S334ter retinal function was more reduced than that of $\mathrm{P} 23 \mathrm{H}$.

Figure 6.1 shows the rates of retinal degeneration in 8 transgenic rat lines carrying a single copy of the transgene for $\mathrm{P} 23 \mathrm{H}$ and S334ter that are available (http://www.ucsfeye.net/mlavailRDratmodels.shtml). The rates of photoreceptor cell loss, described as the degeneration curve values for the mean outer nuclear layer (ONL) are shown for both $\mathrm{P} 23 \mathrm{H}$ and $\mathrm{S} 334$ ter genetic construction on a scale up to two months of age. Lines 3 and 4, of $\mathrm{P} 23 \mathrm{H}$ and S334ter, respectively, were chosen because they have a similar pattern and degree of retinal degeneration over time. The retinal degeneration of these lines is already significant at the age of 8 weeks (data not shown), which allows for an easy comparison between different experimental treatments at early or later stages. 
Figure 6.1 - Rates of degeneration in mutant rhodopsin transgenic rats.
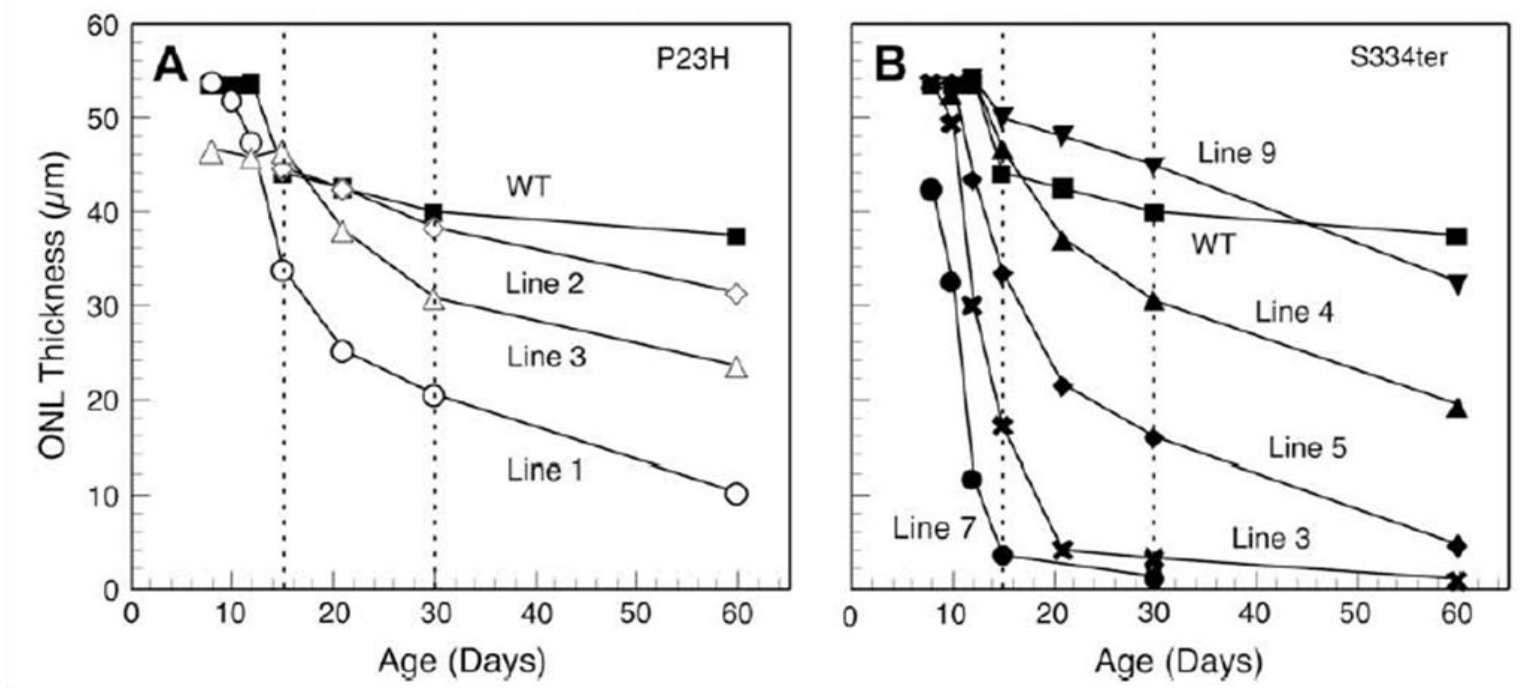

Outer nuclear layer (ONL) thickness, which is proportional to the number of photoreceptor nuclei, in the $\mathrm{P} 23 \mathrm{H}$ (left) and S334ter (right) mutant rhodopsin transgenes.

SOURCE: Figure is a courtesy of Matthew M. LaVail (Pennesi, 2008). With permission.

Representative retinal sections of the P23H-3 and S334ter-4 rats are shown in Figures 6.2 and 6.3, respectively. Samples were collected every month, starting when the rats were 30 days old until 150 days. In agreement with the degeneration rates shown in Figure 6.1, the overall photoreceptor layer decreases over time for both lines of rats. However, the degeneration rate is faster for S334ter rats, where less than three layers remains at the most preserved regions, while other regions present only one layer or even no photoreceptors at all, for 150 days old rats.

Figure 6.2 - Retinal degeneration in the mutant rhodopsin transgenic rat P23H.
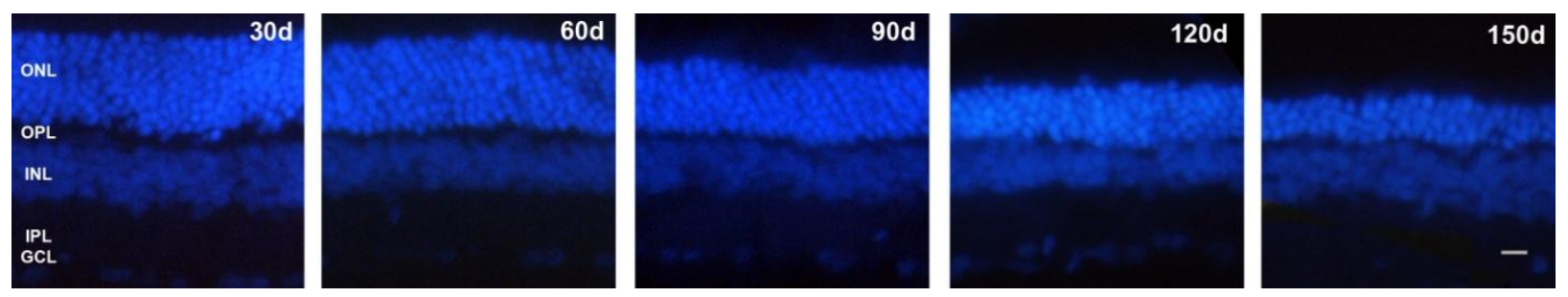

Retinal cross sections at P30, P60, P90, P120 and P150 rats stained with DAPI, a nuclear marker, to visualize the outer nuclear layer. ONL, outer nuclear layer; OPL, outer plexiform layer; INL, inner nuclear layer; IPL, inner plexiform layer; GCL, ganglion cell layer. Scale bar: $10 \mu \mathrm{m}$.

SOURCE: Balen, 2012 
Figure 6.3 - Retinal degeneration in the mutant rhodopsin transgenic rat S334ter.
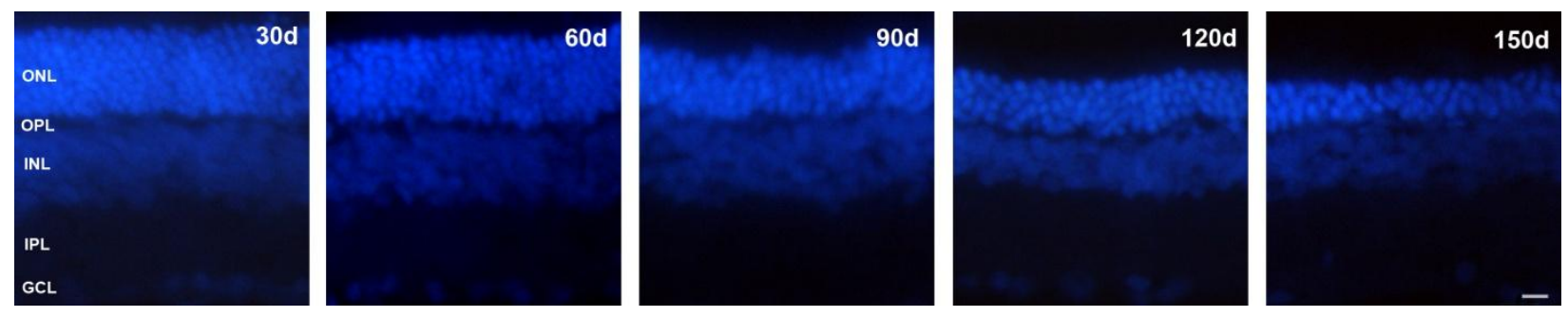

Retinal cross sections at P30, P60, P90, P120 and P150 rats stained with DAPI, a nuclear marker, to visualize the outer nuclear layer. ONL, outer nuclear layer; OPL, outer plexiform layer; INL, inner nuclear layer; IPL, inner plexiform layer; GCL, ganglion cell layer. Scale bar: $10 \mu \mathrm{m}$.

SOURCE: Balen, 2012

\subsubsection{ERG}

The electroretinogram mass electrical response (ERG) is defined as the light-induced potential change that is related to light-induced electrical activity within the retina (Perlman, 2011). ERG measures the electrical responses of various cell types in the retina, such as the photoreceptors (rods and cones) and inner retinal cells (bipolar and amacrine cells), in response to a light stimulus. The ERG consists of four waves (a, b, c, and d) (Perlman, 2011). These are dependent on the animal, state of the retina, as well the applied technique. While a-, b- and cwaves represent a response to a light stimulus, the d-wave happens after the stimulus has finished. The a-wave and the b-wave are the most sensitive and most prominent elements of an ERG (Dong and Hare, 2002). The small initial a-wave is originated by photoreceptor activity. The large positive b-wave reflects mainly ON bipolar cell depolarization (Fleisch et al., 2008).

According to the International Society for Clinical Electrophysiology of Vision (ISCEV) (Marmor et al., 1989), both the amplitude and the implicit time should be measured for a selected ERG signal. The most frequent measurements are: 1) the b-wave amplitude of the "rod response", 2) the maximal combined response and single flash "cone response", 3) the b-wave time-to-peak of the single flash "cone response" and 4) flicker response. Figure 6.4 shows the measurement procedures according to current convention. The a-wave amplitude is measured from baseline to a-wave trough, the b-wave amplitude is measured from a-wave trough to b-wave peak, and the b-wave time-to-peak is measured from the time of the flash to the peak of the wave. 
Figure 6.4 - Diagram illustrating the five basic ERG responses defined by the ISCEV (1999 update).
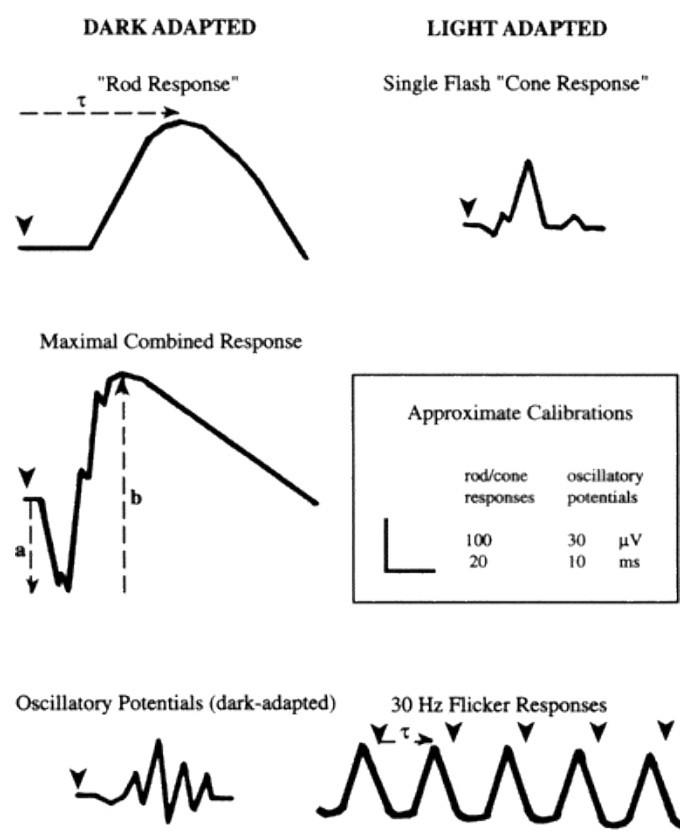

The time of stimulus (flash) is indicated by the large arrowheads. Dotted arrows exemplify how to measure time-topeak ( $t$, implicit time), a-wave amplitude and $b$-wave amplitude.

SOURCE: Figure taken from (Marmor et al., 1989). With permission.

Figure 6.5 show a detailed example of how amplitude and latency of a- and b-waves are measured. 
Figure 6.5 - Example of ERG parameters that are normally measured in the ophthalmic clinic for electrodiagnosis.

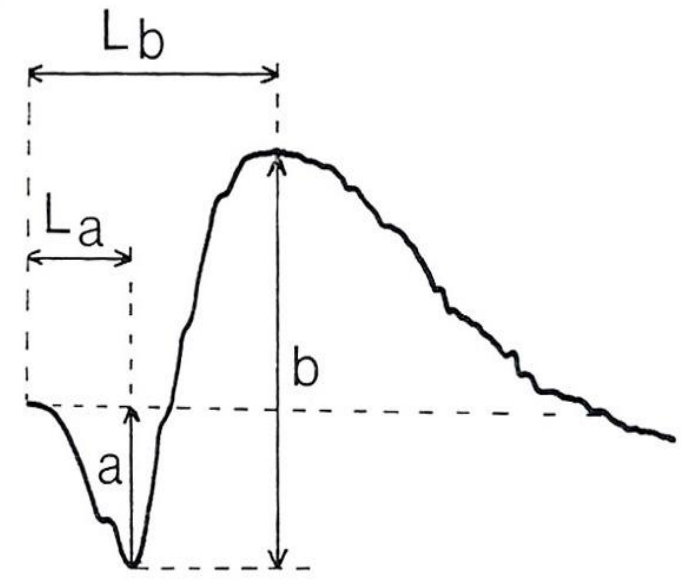

The size of a-wave is measured from the baseline to the trough of the wave. b-wave size is measured from the trough of the a-wave to the peak of the b-wave. The latency or time-to-peak for both waves, $\mathrm{La}$ and $\mathrm{Lb}$, is determined from stimulus onset to the trough or peak of the waves.

SOURCE: Figure adapted from (Perlman, 2011). With permission.

\subsubsection{Treatment of $\mathrm{P} 23 \mathrm{H}$ rats with $\mathrm{Ce} 6$}

\subsubsection{Dose dependent effect of Ce6 on photoreceptor cell death}

Different doses of Ce6 were tested $(2,10$ and $20 \mathrm{mg} / \mathrm{Kg})$ to verify whether Ce6 had an effect on retinal damage prevention and if this effect was dose-dependent. Rats were intraperitoneally treated with Ce6 every 2 days from postnatal day 35 (P35) until P60. Two groups of control rats were also treated with: a) phosphate buffered saline solution (0.1 M PBS, $\mathrm{pH}$ 7.2, negative control) and b) DMTU, which has shown to exert a positive effect on retinal damage prevention ((Organisciak et al., 1992), positive control).

Confocal micrographs of transverse retinal sections showing photoreceptor cell layers of PBS, Ce6 and DMTU treated rats are shown in Figure 6.6. Comparison of the outer nuclear layer thickness of rats subjected to different treatments suggested that Ce6 exerted an effect on the photoreceptor cells hereditary degeneration.

Treatment with Ce6 at 2 and $20 \mathrm{mg}$ has shown similar results, whereas the results of the $10 \mathrm{mg}$ group were not consistent. In DMTU treated rats, a homogeneous and thicker outer nuclear layer was observed. 
Figure 6.6 - Retinal sections of P23H rats treated with PBS, different concentrations of Ce6 and DMTU.
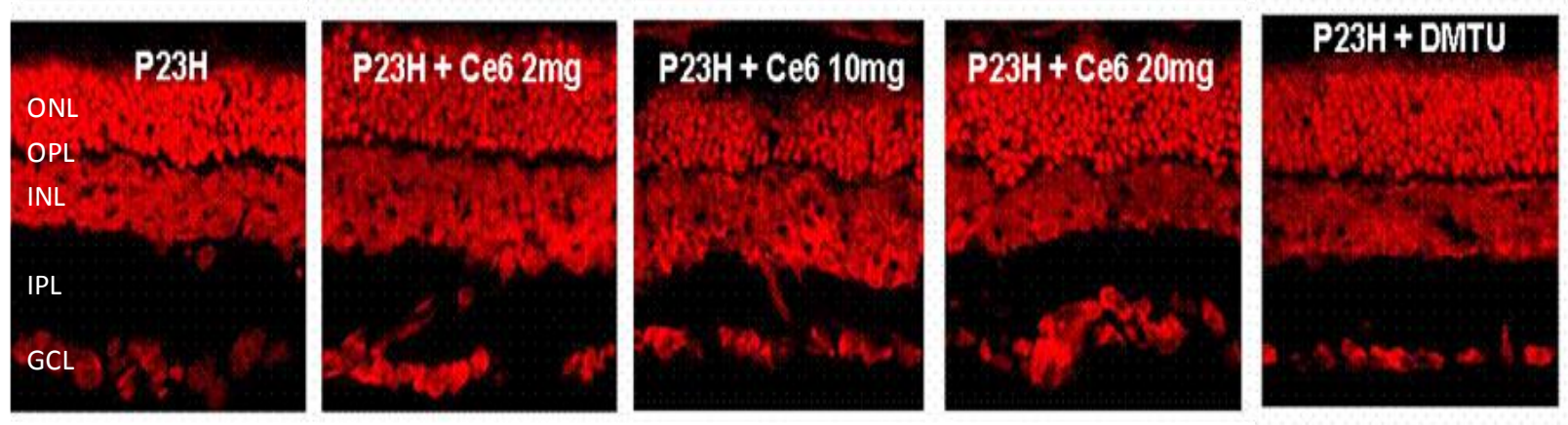

PBS treated rats $(\mathrm{n}=2)$, Ce6 treated with 2,10 and $20 \mathrm{mg} / \mathrm{kg}(\mathrm{n}=2$ for each concentration) and DMTU treated with $500 \mathrm{mg} / \mathrm{kg}(\mathrm{n}=2)$. Nuclear marker: Propidium Iodide. ONL: outer nuclear layer; OPL: outer plexiform layer; INL: inner nuclear layer; IPL: inner plexiform layer; GCL: ganglion cell layer.

SOURCE: Balen, 2012

\subsubsection{Morphological analysis}

Since treatment with Ce6 appears not to be dose dependent, at least in the range tested, all the experiments were performed using $2 \mathrm{mg} / \mathrm{kg}$. Ce6 was administered every second day from P20 until 120 days, and rats were sacrificed at three different time points (P60, P90 and P150). The histological analysis consisted in morphological comparison between rats that were treated and non-treated with Ce6 (Section 6.2.1.3 and 6.2.1.4) and ONL measurements (6.2.1.5).

Comparison between non-treated and treated DAPI-stained retinal sections over the months revealed a slight preservation of the ONL for the treated $\mathrm{P} 23 \mathrm{H}$ rats at P90 (Figure 6.7). At P60 PBS-treated rats ONL seems to have more retinal layers than Ce6 treated. 
Figure 6.7 - Comparison of P23H rats Ce6 treated and non-treated at P60, P90 and P150.
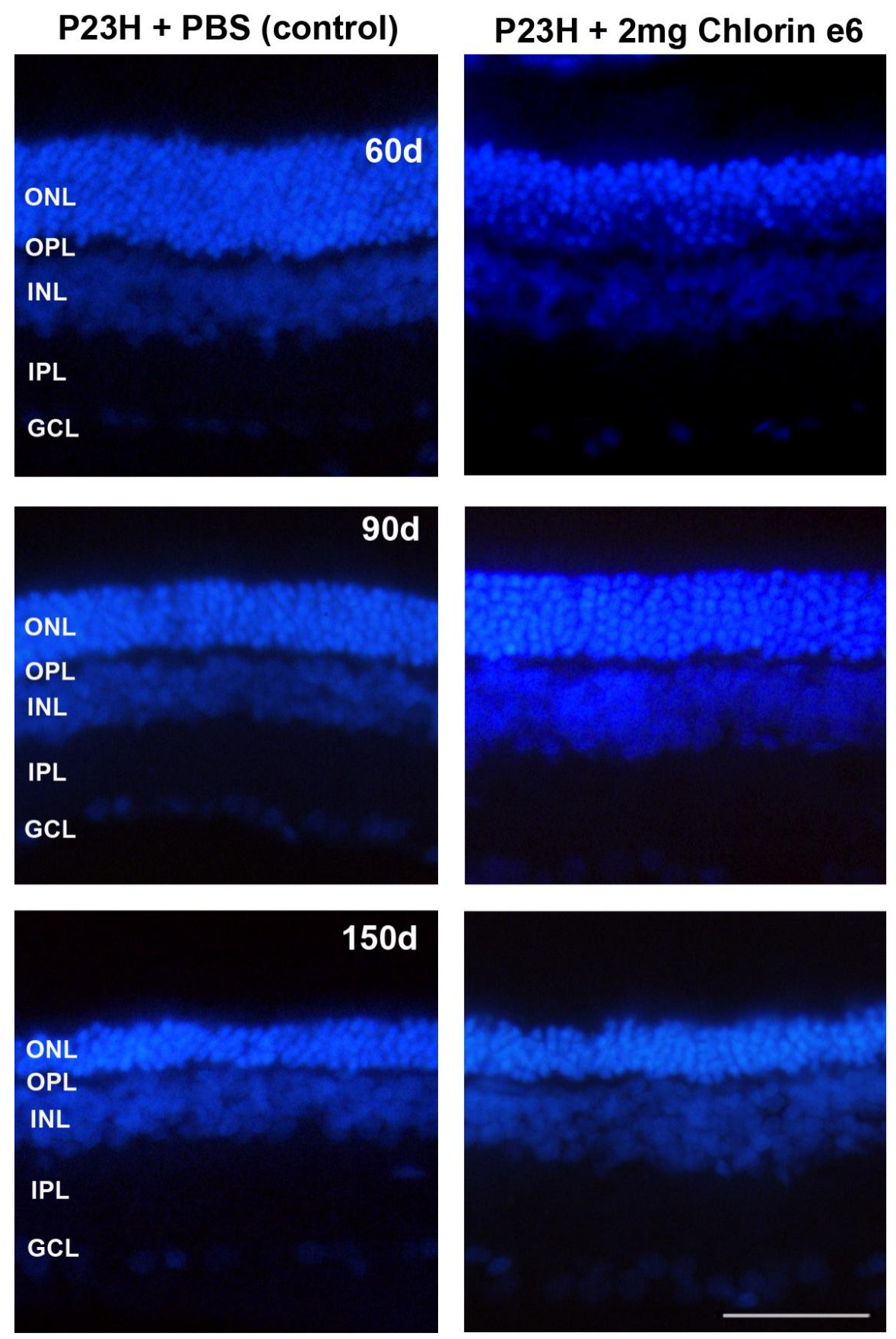

Retinal cross sections of $\mathrm{P} 23 \mathrm{H}$ rats non-treated (left side) and Ce6 treated (right side) stained with DAPI, a nuclear marker, to visualize the outer nuclear layer. ONL, outer nuclear layer; OPL, outer plexiform layer; INL, inner nuclear layer; IPL, inner plexiform layer; GCL, ganglion cell layer. Scale bar: $50 \mu \mathrm{m}$.

SOURCE: Balen, 2012 
Figure 6.8 shows the mean ONL thickness and standard deviation of non-treated and treated $\mathrm{P} 23 \mathrm{H}$ rats with $\mathrm{Ce} 6$. Measurements were obtained from all regions of the eye (see Sections 2.4.6) and averaged to give a unique value for comparison at P60, P90 and P150. In nontreated rats, P30 and P120 were also included in the analysis. At P90, despite the large standard deviation values, the retinas of treated animals were more preserved, and at P150 they appear to be similar. Retinal degeneration was smaller at the control group at P60.

Figure 6.8 - Mean ONL thickness of the Ce6 treated and non-treated P23H rats.

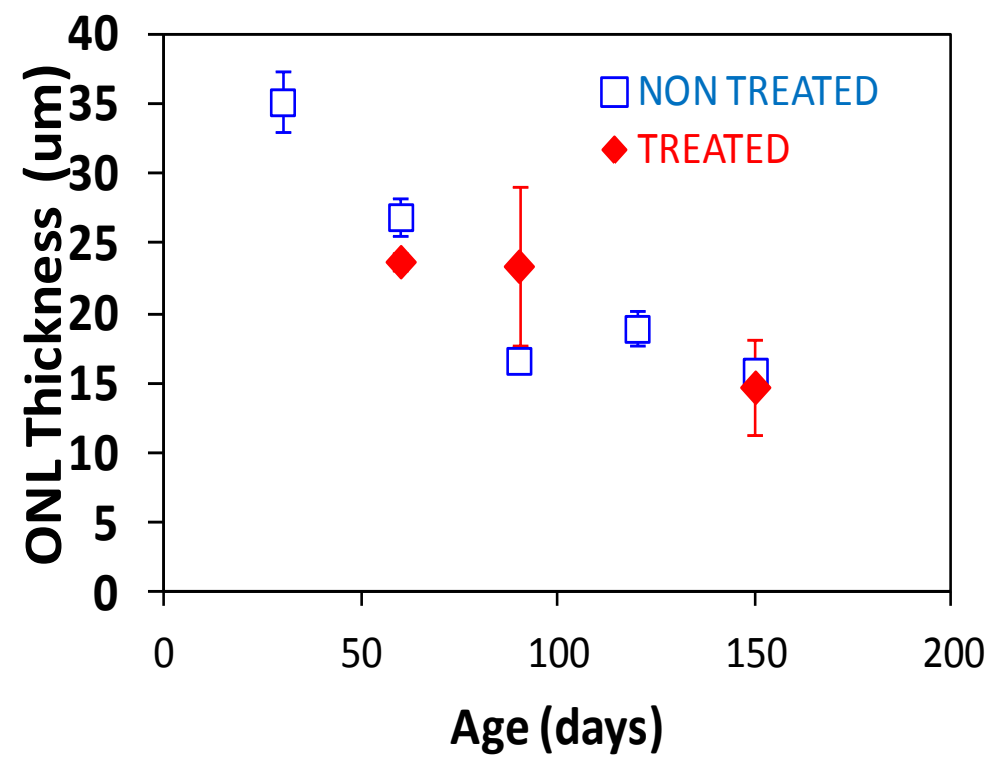

Data points are the mean including standard deviation of the total measurements of the retina. For illustration a data point of a non-treated rat at P30 and P120 was also plotted. Number of animals (n) of non-treated rats at different age: 30 (3); 60 (3); 90 (3); 120 (4) and 150 (3), and treated rats: 60 (2); 90 (4) and 150 (2).

SOURCE: Balen, 2012

Figure 6.9, shows the average of all measurements performed in the ONL of the $\mathrm{P} 23 \mathrm{H}$ rats. At P60, retinal degeneration appears to be in overall similar for the two groups. Ce6-treated retinas are more preserved than non-treated at 90 days. In addition, it can be seen that essentially no retinal degeneration has occurred for $\mathrm{Ce} 6$ treated rats from P60 to P90, contrary to that observed for non-treated rats. Treatment was stopped at P120. The final data point, P150, shows that the degeneration of both treated and non-treated was similar. 
Figure 6.9 - Measurements of ONL thickness plotted as a function of the distance from the optical nerve head.

P23H Non treated

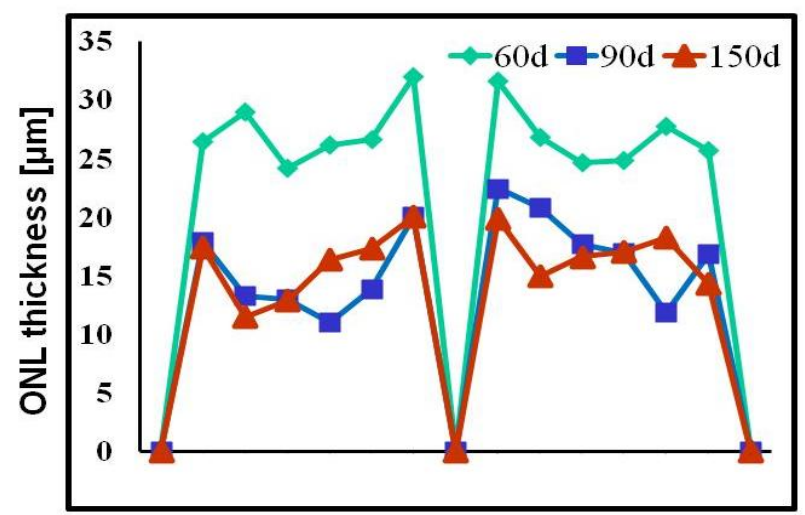

Distance from optical nerve
P23H Treated

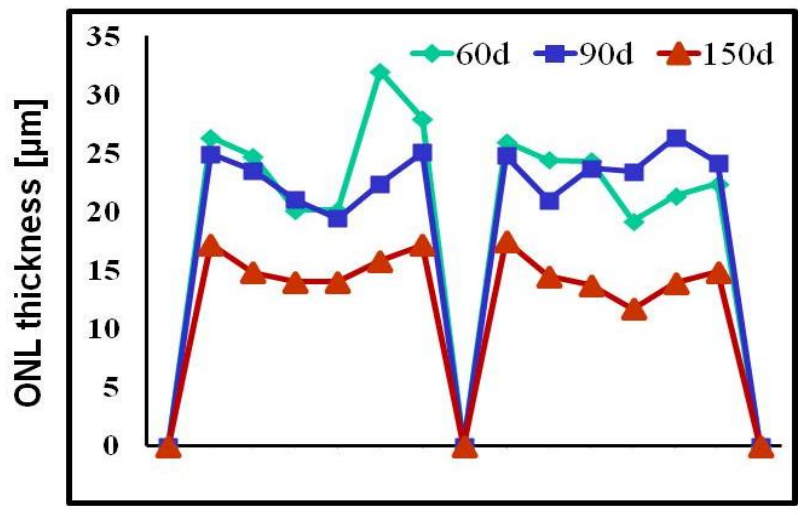

Distance from optical nerve

Left side of the optical nerve: dorsal side of eye. Right side of the optical nerve: ventral part of the eye. Values are an average of 3 measurements for each area. Number of animals (n) of non-treated rats at different age: 60 (3); 90 (3); and 150 (3), and treated rats: 60 (2); 90 (4) and 150 (2).

SOURCE: Balen, 2012

\subsubsection{Bipolar cell morphology}

The dendritic terminals of $\mathrm{ON}$ rod bipolar cells in rat retinas are connected to rod spherules through a large dendritic arbor in the OPL and their axons run into the IPL. For review (Fernandez-Sanchez et al., 2011). In order to evaluate whether Ce6 could preserve native photoreceptor morphology, cross sections of the retina were submitted to immunofluorescence with anti-PKC alpha (anti-protein kinase C), a marker for rod bipolar cells (Figure 6.10 and 6.11). All rod bipolar cells and a subtype of amacrine cells were labeled with anti-PKC. In control retinas, a general disorganization and a loss of bipolar cell bodies with retraction of their dendrites was observed, while rod bipolar cells and their dendrites were more preserved in Ce6 treated rats. 
Figure 6.10 - Cross sections of the retina showing immunofluorescence for anti-PKC alpha, a marker for rod bipolar cells in non-treated (A-C) and Ce6 treated (D-F).
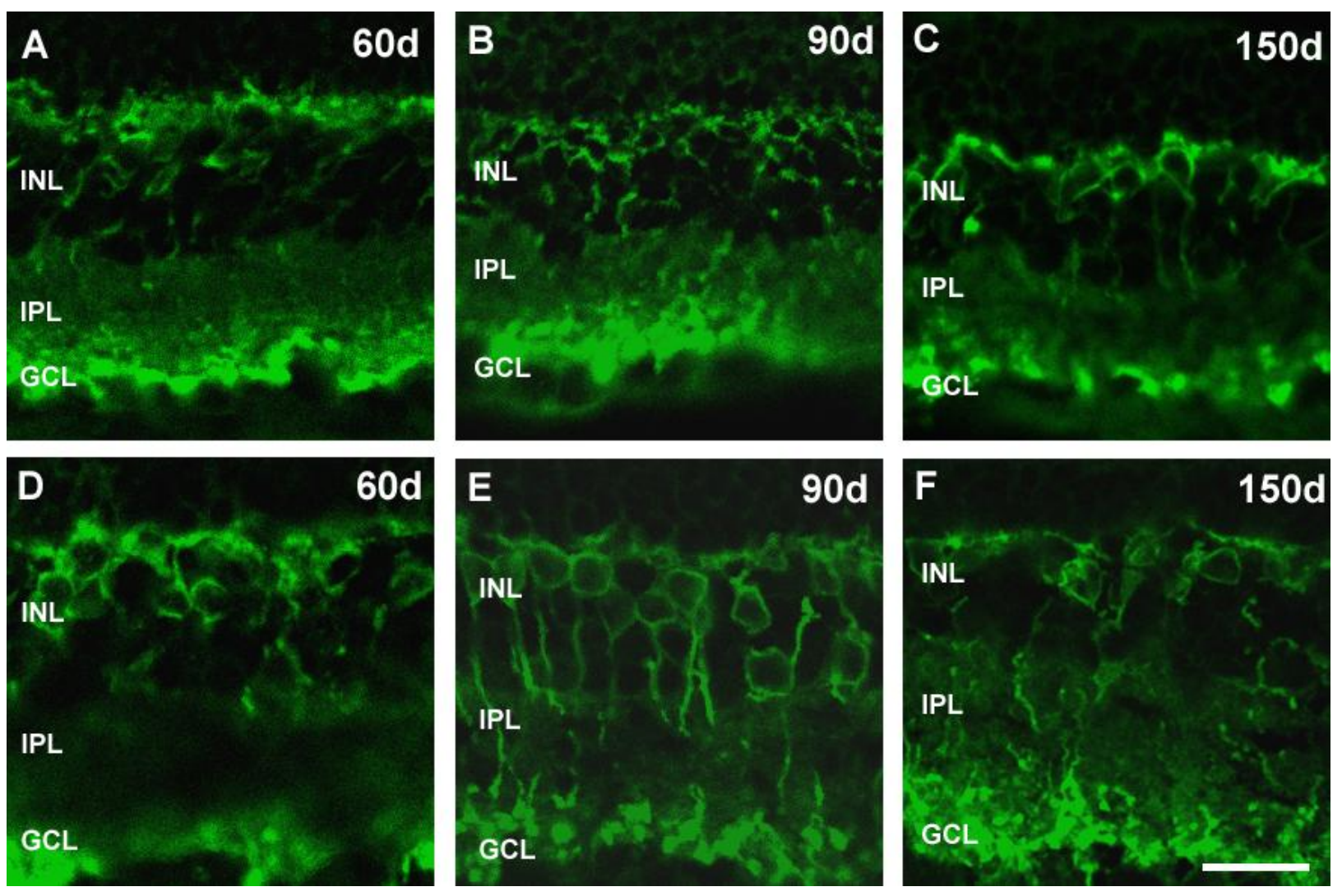

ONL, outer nuclear layer; OPL, outer plexiform layer; INL, inner nuclear layer; IPL, inner plexiform layer; GCL, ganglion cell layer. Scale: $30 \mu \mathrm{m}$.

SOURCE: Balen, 2012 
Figure 6.11 - Cross sections of the retina showing immunofluorescence for anti-PKC alpha, a marker for rod bipolar cells in non-treated (left column) and Ce6 treated (right column) at P90.
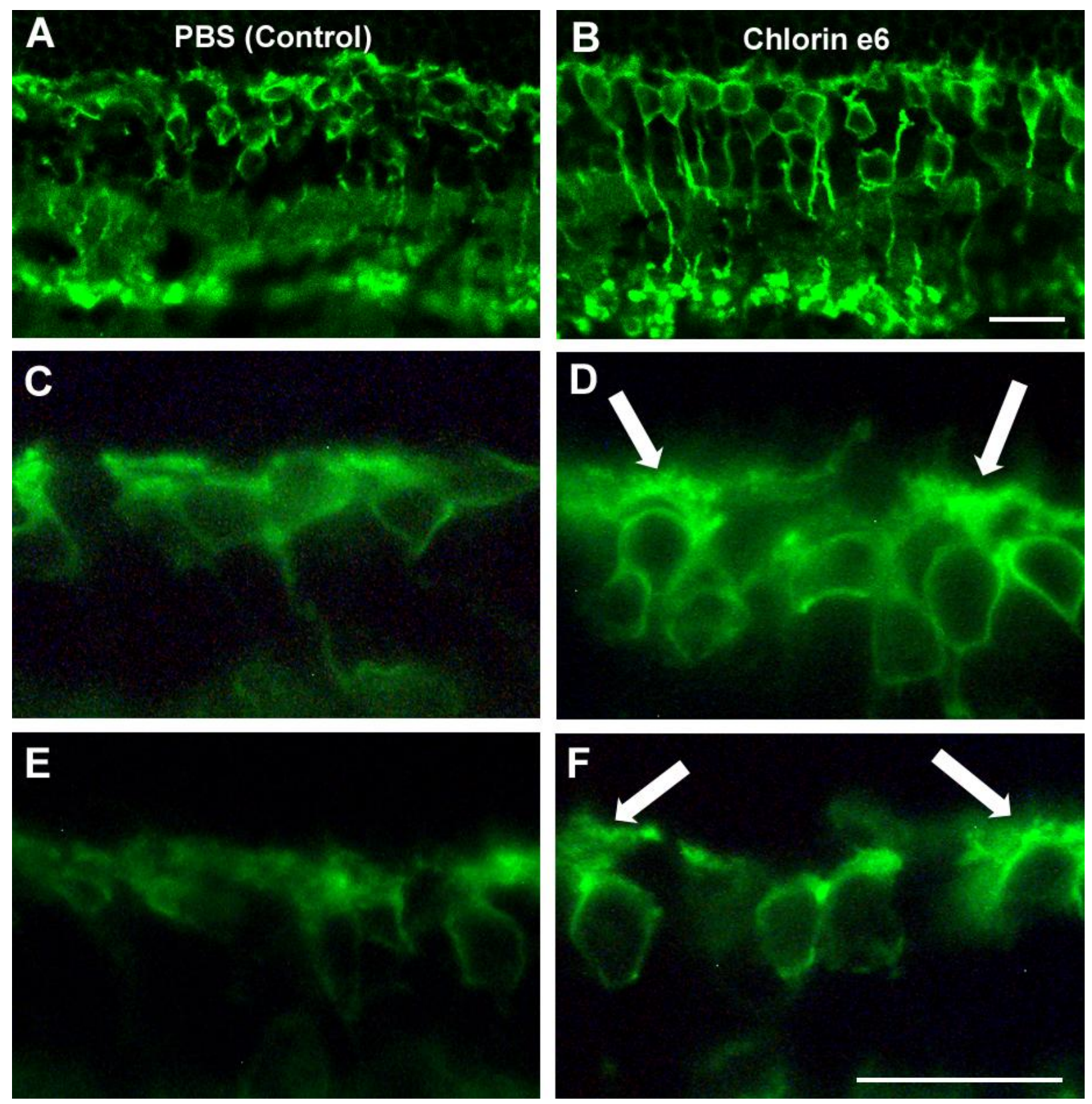

Details of the preserved rod bipolar cell arborization in the OPL can be observed (arrows). ONL, outer nuclear layer; OPL, outer plexiform layer; INL, inner nuclear layer; IPL, inner plexiform layer; GCL, ganglion cell layer. Scale: $30 \mu \mathrm{m}$.

SOURCE: Balen, 2012 


\subsubsection{Opsin localization}

In order to investigate the subcellular localization of $\mathrm{P} 23 \mathrm{H}$ opsin of rats that were treated with Ce6, the C-terminal opsin antibody 1D4 was used and the retinal sections of $\mathrm{P} 23 \mathrm{H}$ rats at ages 60 (A-C), 90 (D-F) and 150 (G-I) are shown in Figure 6.12 in comparison to untreated rats. 
Figure 6.12 - Rhodopsin immunolabeling in non-treated P23H rats.
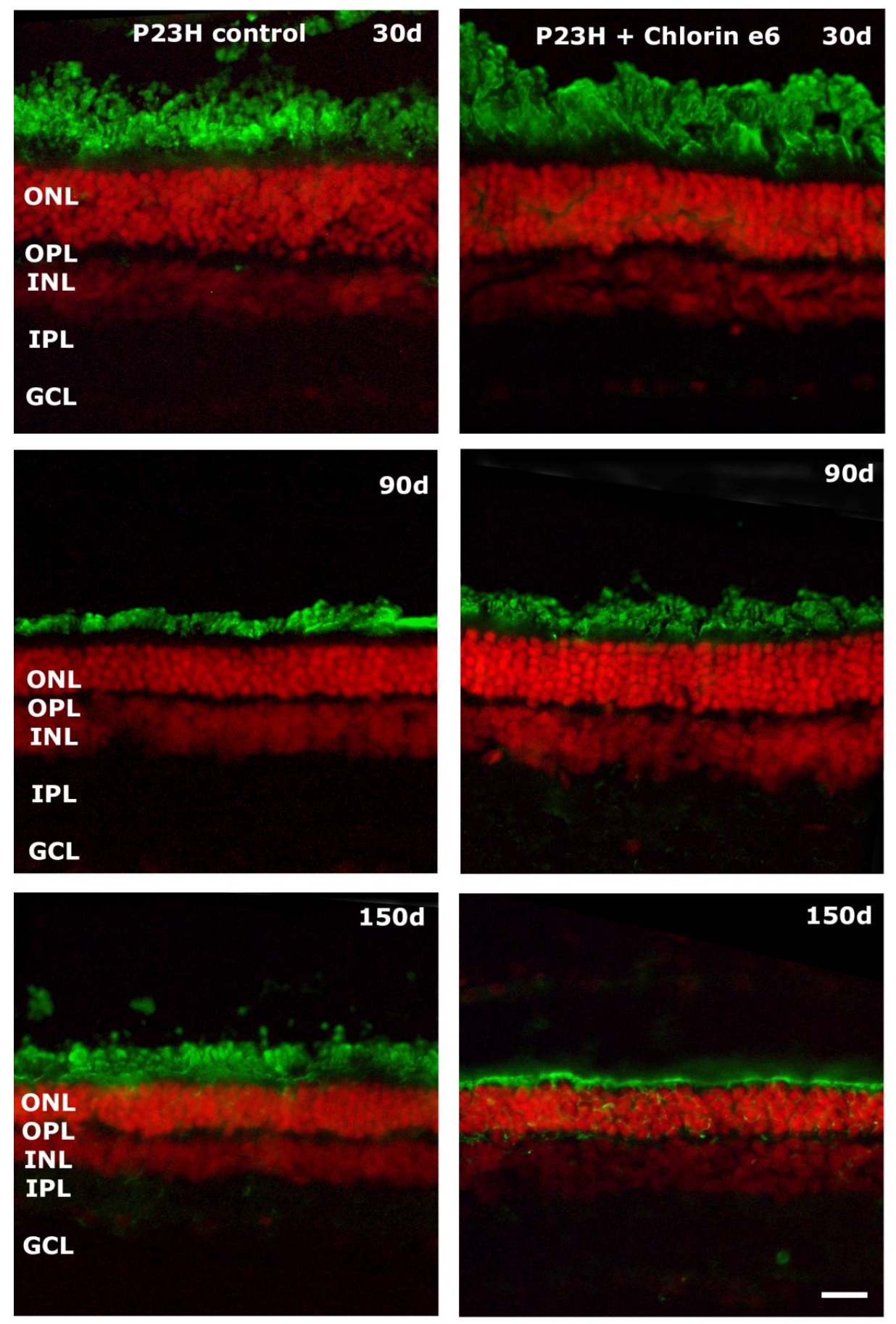

Retinal cross-sections of $\mathrm{P} 23 \mathrm{H}$ rats non-treated and treated with Ce6 were stained with the antibody 1D4, an opsin marker. The 1D4 antibody reacts with the opsin protein that is localized in the ROS. Left column represents the retinal sections of non-treated rats and right column retinal sections of Ce6 treated rats at P60, P90 and P150. Scale $30 \mu \mathrm{m}$. 


\subsubsection{ERG analysis of P23H rats after treatment with Ce6}

In order to evaluate the effect of $\mathrm{Ce} 6$ on functional activity of $\mathrm{P} 23 \mathrm{H}$ rat photoreceptors, scotopic, photopic and flicker ERG responses were recorded every month, starting at P60, followed by measurements at P90, P120 and P150 (Section 6.2.1.6). ERG measurements were performed just before the rats were sacrificed, but none of them was sacrificed at P120. No baseline control ERG measurements were performed at P20, before treatment was started.

Figure 6.13 shows an example of the ERG response obtained for one rat of each nontreated and treated groups at different ages. $\mathrm{P} 23 \mathrm{H}$ non-treated and treated rats showed a continuous decrease of ERG response over the period investigated. In general, ERG responsiveness of Ce6 treated rats appeared to be much less deteriorated than non-treated, showing a smaller decrease over the months tracked.

Figure 6.13 - Example of one rat response of ERGs total field (signal in $\mu \mathrm{V}$ ) of non-treated versus Ce6 treat animals.

P23H Non treated $\quad$ P23H Treated

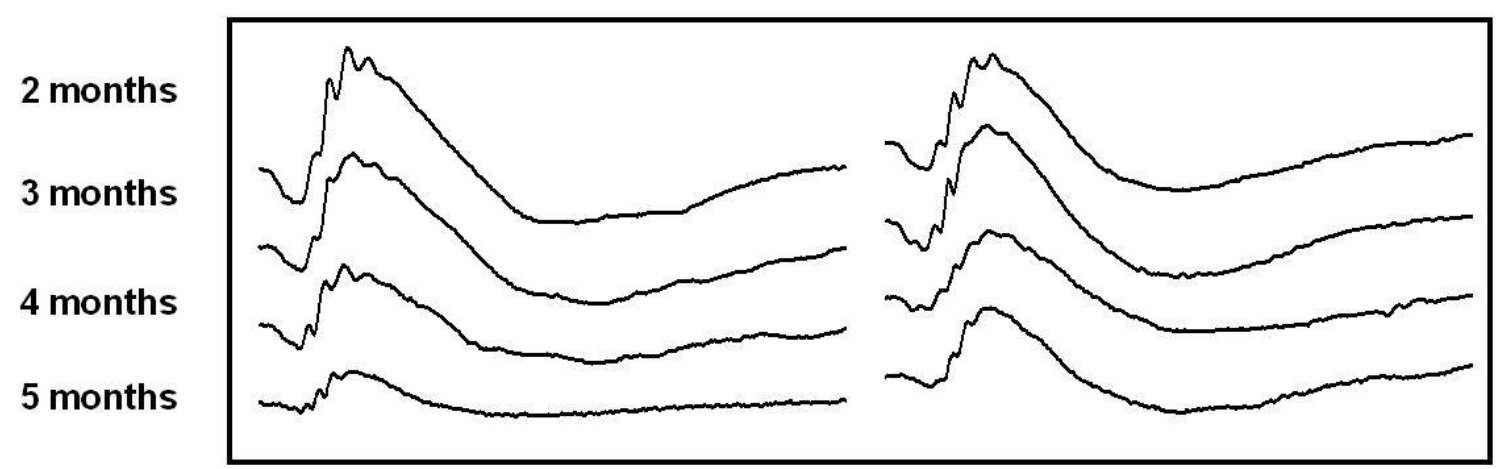

Treatment consisted of intraperitoneal injections every second day, from P20 until P120. ERG data was collected at P60, P90, P120 and at P150. Each line represents the data collected of only one rat (not average of all rats).

SOURCE: Balen, 2012

\section{a-wave:}

The scotopic ERG a-wave amplitude as a function of both light intensity, varying intensity of filters, and rat age, at the maximum intensity measured and standard deviation values, are shown in Figure 6.14 and 6.15, respectively. Figure 6.14 shows the overall ERG response at 
different light intensities. At P60 the response was higher for non-treated as compared to treated ones, but smaller at P90. At P120 and P150 the average value appears to be similar.

Figure 6.14 - ERG a-wave responses of P23H rats.

\section{Scotopic ERG - Amplitude of a-wave}
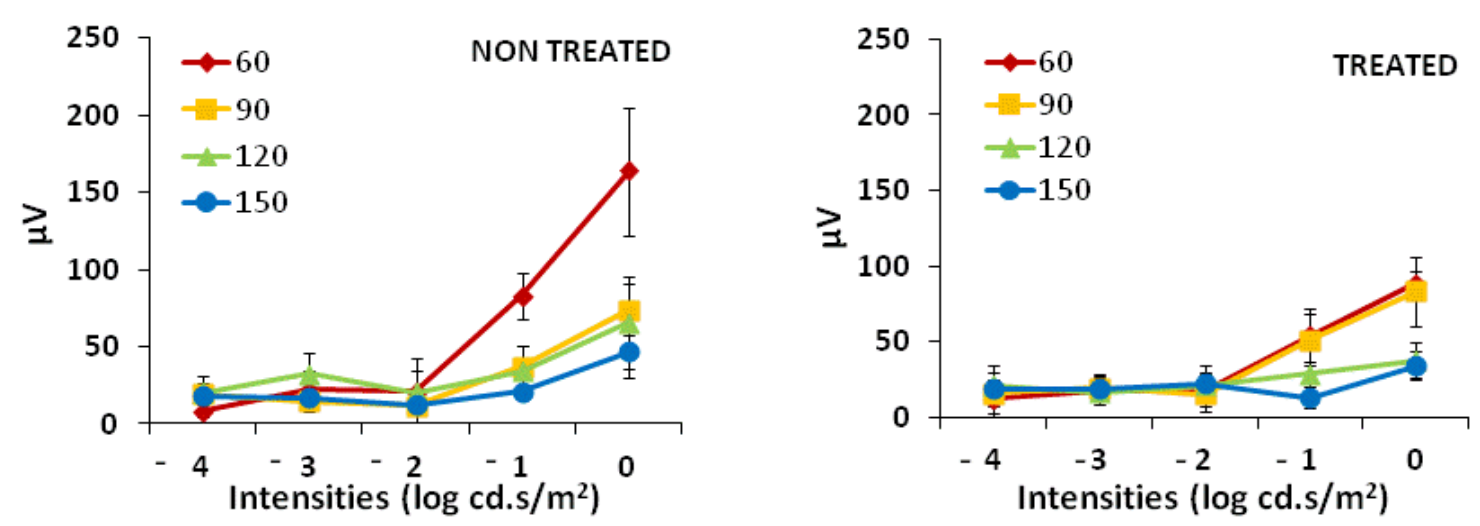

Average ERG response with standard deviation of non-treated (left) and treated (right) P23H rats as a function of light intensity at different ages, 60, 90, 120 and 150 days old. Number of animals (n) of non-treated rats at different age: 60 (5); 90 (6); 120 (4) and 150 (4), and treated rats: 60 (8); 90 (10); 120 (4) and 150 (3).

SOURCE: Balen, 2012

Measurements of the amplitude response of the $\mathrm{P} 23 \mathrm{H}$ rats treated and non-treated as a function of age at the maximum intensity measured are shown on Figure 6.15. Non-treated rats have shown a larger amplitude at the first data point collected, P60, as seen by the comparison of the averages (Figure 6.15A). However at P90, P120 and P150 the scotopic responses were similar for both treated and non-treated rats. These demonstrated that the overall decay of the a-wave was higher for the non-treated group, which suffered a larger deterioration of the a-wave as compared to treated rats from P60 to P90. 
Figure 6.15 - Average of a-wave amplitude (maximum scotopic response: $30 \mathrm{cd.s} / \mathrm{m} 2$ ).

\section{Scotopic ERG - Amplitude of a-wave}

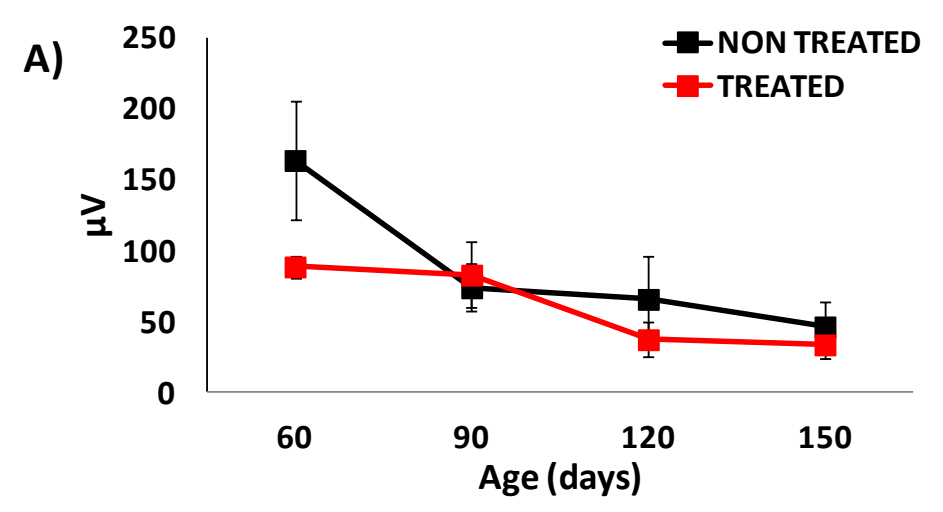

B)

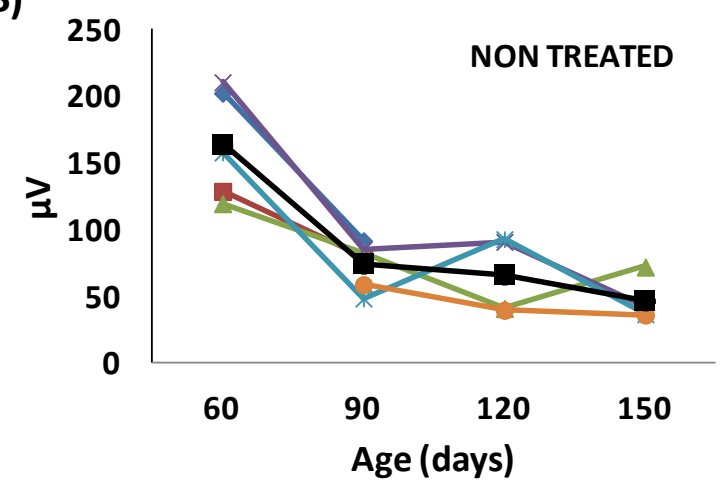

C)

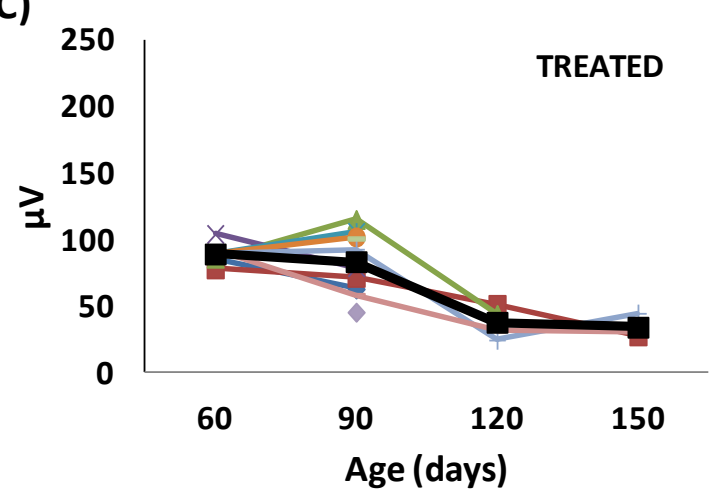

Average with standard deviation of a-wave amplitude for treated and non-treated animals (A). Distribution of each individual and average of the group (black line) for (B) non-treated and (C) treated $\mathrm{P} 23 \mathrm{H}$ rats. Number of animals (n) of non-treated rats at different age in days: 60 (5); 90 (6); 120 (4) and 150 (4), and treated rats: 60 (8); 90 (10); 120 (4) and $150(3)$.

SOURCE: Balen, 2012

The scotopic ERG latencies of $\mathrm{P} 23 \mathrm{H}$ non-treated and treated rats were also recorded as shown in Figure 6.16. No statistically significant differences were observed among different ages in each group or between non-treated and treated rats. 
Figure 6.16 - Average of a-wave latency (maximum scotopic response: $30 \mathrm{cd.s} / \mathrm{m}^{2}$ ).

\section{Scotopic ERG - Latency of a-wave}

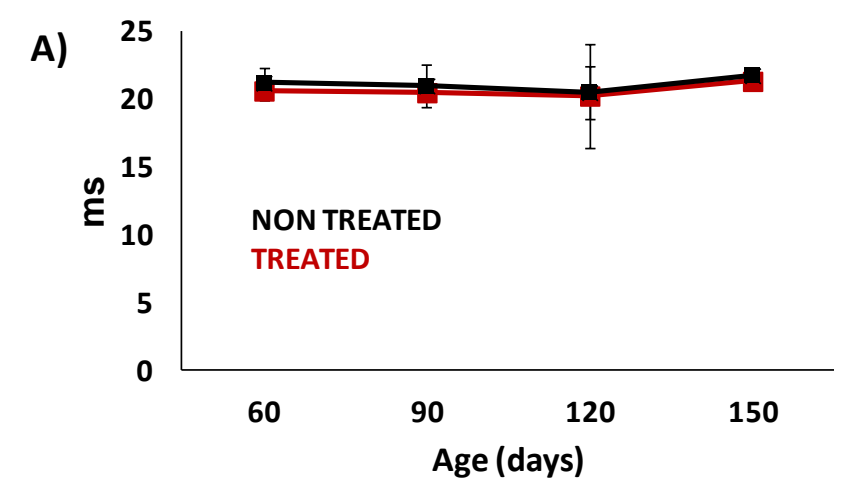

B)

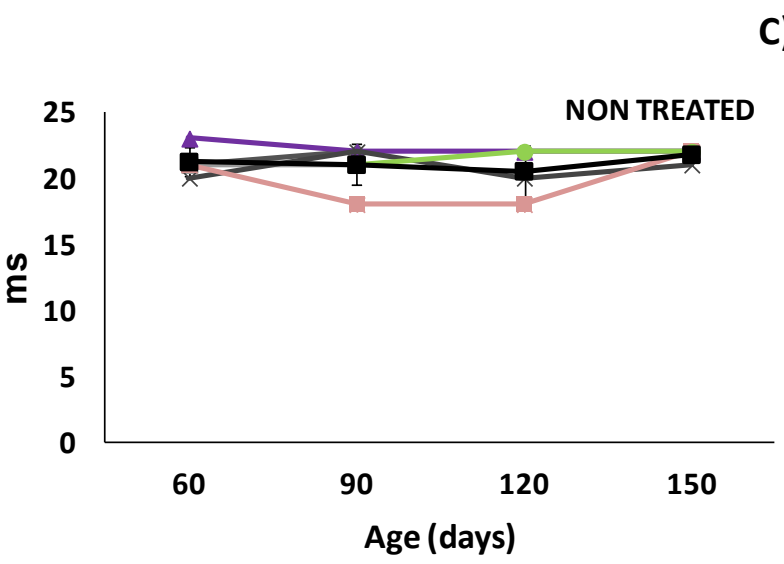

C)

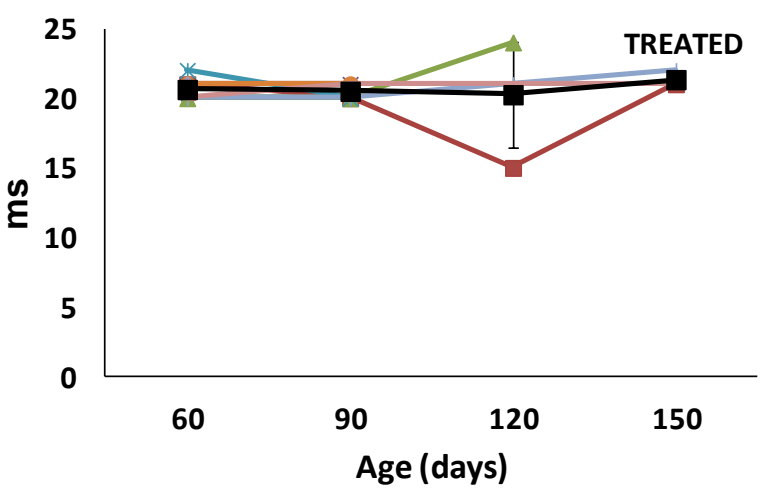

Average with standard deviation of a-wave latency for treated and non-treated animals (A). Distribution of each individual and average of the group (black line) for (B) non-treated and (C) treated $\mathrm{P} 23 \mathrm{H}$ rats. Number of animals (n) of non-treated rats at different age in days: 60 (5); 90 (6); 120 (4) and 150 (4). And treated rats: 60 (8); 90 (10); 120 (4) and $150(3)$.

SOURCE: Balen, 2012

\section{b-wave:}

The average response of scotopic ERG b-wave of non-treated and Ce6 treated rats as a function of different filters is shown in Figure 6.17. A linear decrease of b-wave ERG response for all non-treated and treated rats was observed along the different ages recorded. Similar to the a-wave, the Ce6 treated rats show lower total b-wave ERG responses at the first data point collected, P60. However, no decrease in response was observed from P60 to P90, contrarily to non-treated rats. Similar responses were observed at P120 and P150 for both groups. 
Figure 6.17 - ERG amplitude response to different filters in P23H rat.

\section{Scotopic ERG - Amplitude of b-wave}
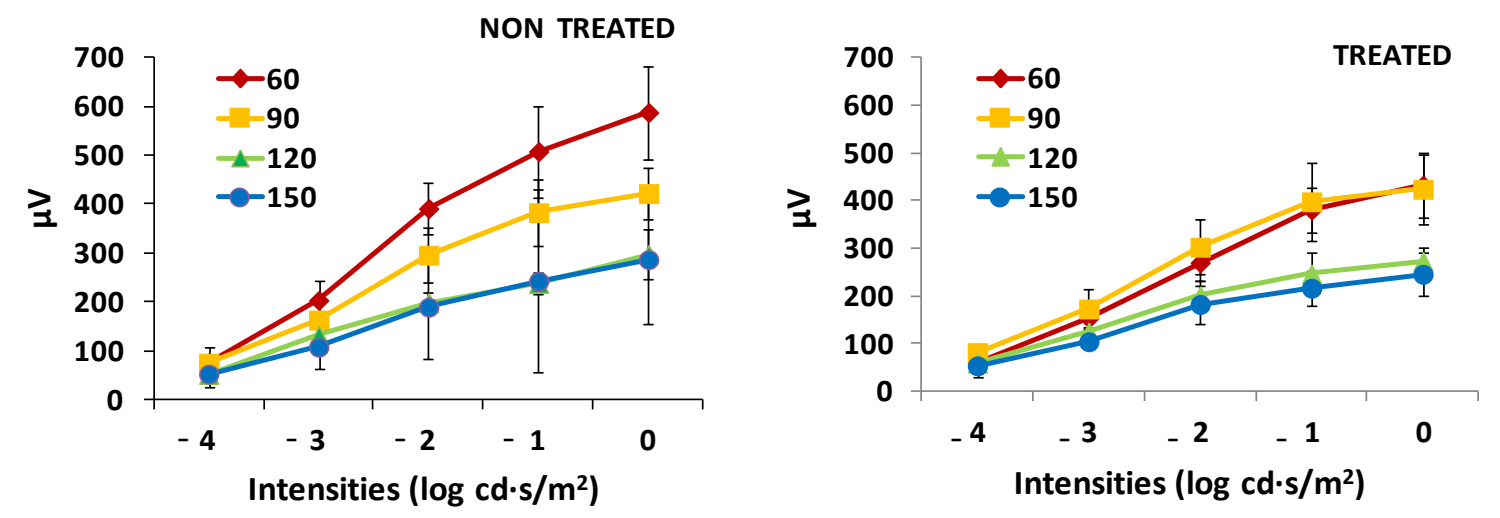

Plotted are the average with standard deviation of the b-wave amplitude of the Ce6 treated and non treated $\mathrm{P} 23 \mathrm{H}$ rats. Number of animals (n) of non-treated rats at different age in days: 60 (5); 90 (6); 120 (4) and 150 (4). And treated rats: 60 (8); 90 (10); 120 (4) and $150(3)$.

SOURCE: Balen, 2012

Figure 6.18 shows a comparison of the average b-wave amplitudes of the non-treated and treated group for the maximum light intensity measured as a function of age. The average response is higher in non-treated rats, and a linear decrease in the response occurs every month. Ce6 treated rats, presented a smaller average values than non-treated rats at the first point, P60. However there was no decrease of the response from 60 to 90 days. For P120 and P150, the average values were similar for both treated and non-treated rats. The total deterioration of the bwave response from P60 to P150 was higher in non-treated rats. 
Figure 6.18 - ERG amplitude response in P23H rat.

\section{Scotopic ERG - Amplitude of b-wave}
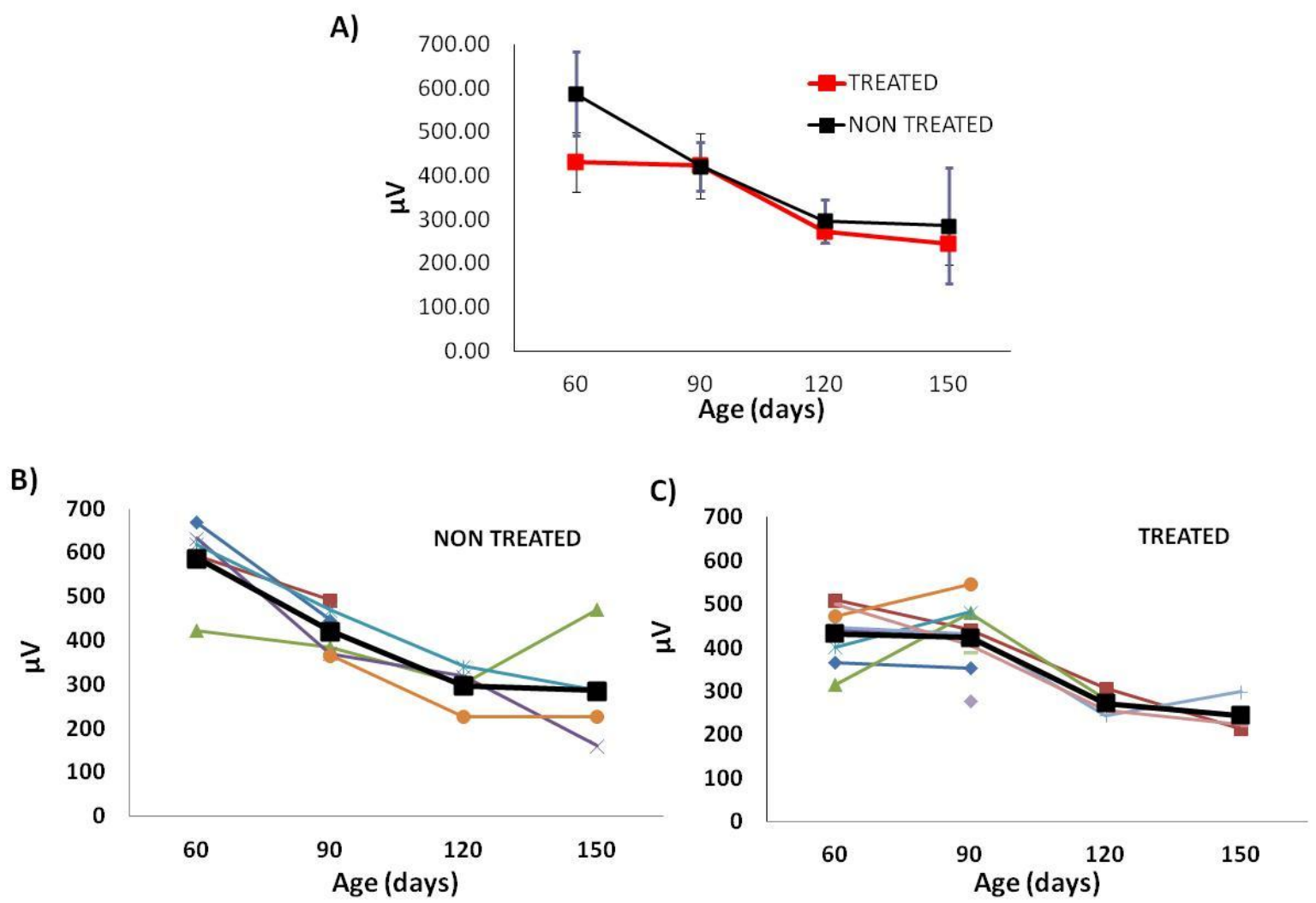

Average with standard deviation of b-wave amplitude of the Ce6 treated and non treated $\mathrm{P} 23 \mathrm{H}$ rats (A) and distribution of b-wave amplitude of each non treated (B) and Ce6 treated (C). Colored lines represent each individual tested. Black line is the average of the group. Number of animals (n) of non-treated rats at different age in days: 60 (5); 90 (6); 120 (4) and 150 (4). And treated rats: 60 (8); 90 (10); 120 (4) and 150 (3).

SOURCE: Balen, 2012

The scotopic ERG latencies of b-waves of P23H non-treated and treated rats were also recorded as shown in Figure 6.19. No difference in the latencies at 60 and 90 days were found for both, non-treated and treated rats. In addition, treated animals show larger latencies at 120 and 150 days old than non-treated ones. 
Figure 6.19 - ERG latency response in P23H rat.

\section{Scotopic ERG - Latency of b-wave}

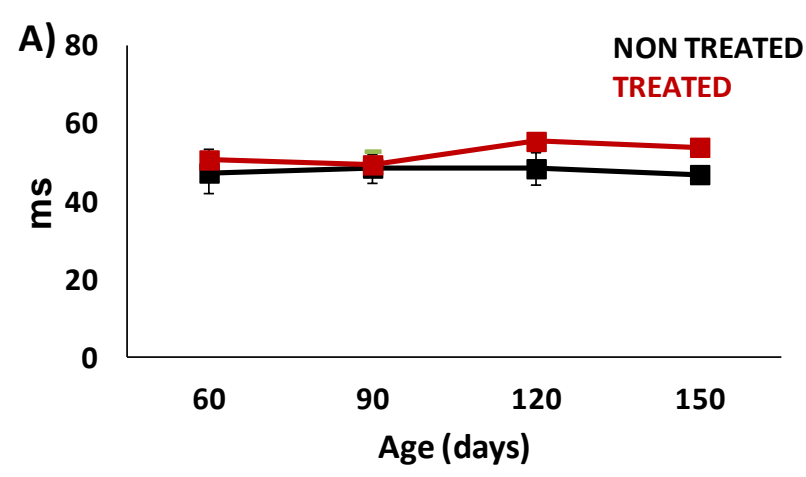

B)
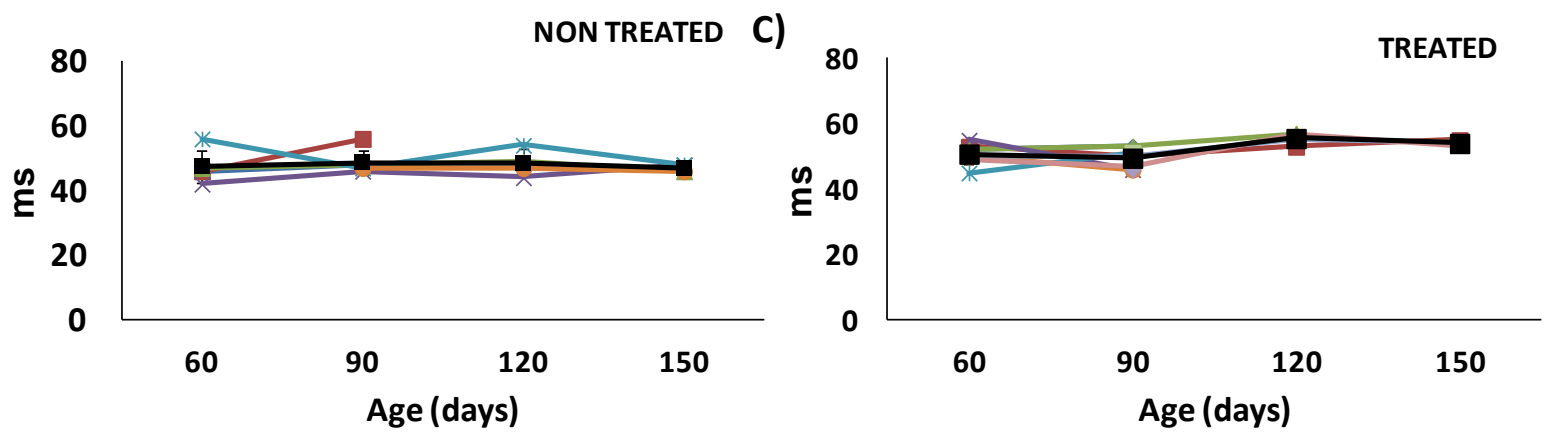

Average with standard deviation of b-wave latency of the Ce6 treated and non treated $\mathrm{P} 23 \mathrm{H}$ rats (A) average and total response of b-wave latency of non-treated rats (B) and Ce6 treated (C). Colored lines represent each individual rat tested. Black line is the average of the group. Number of animals (n) of non-treated rats at different age in days: 60 (5); 90 (6); 120 (4) and 150 (4). And treated rats: 60 (8); 90 (10); 120 (4) and 150 (3).

SOURCE: Balen, 2012

\section{Photopic ERG response:}

ERG responses in $\mathrm{P} 23 \mathrm{H}$ rats under photopic conditions (Figure 6.20) were similar for non-treated and treated rats at the first data point collected, 60 days old, while slightly reduced responses were observed for non-treated rats at ages 90 and 120 days. Rats 150 days old showed similar values independently of prior treatment. 
Figure 6.20 - Photopic ERG response in P23H rat.

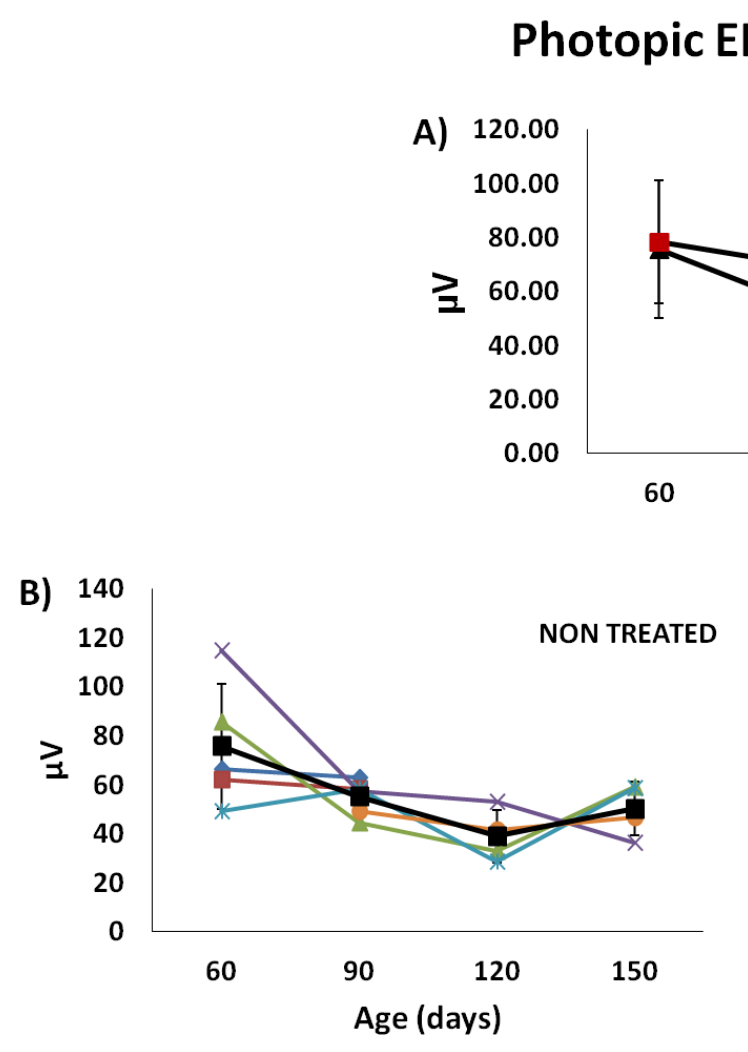

Average with standard deviation of the ERG photopic response of the Ce6 treated and non-treated $\mathrm{P} 23 \mathrm{H}$ rats (A) average and total response of non-treated rats (B) and Ce6 treated (C). Colored lines represent each individual tested. Black line is the average of the group.

SOURCE: Balen, 2012

\section{Flicker ERG response:}

Analysis of cone activity was also assessed by Flicker ERG. Figure 6.21 shows an example of the ERG flicker response amplitudes in $\mathrm{P} 23 \mathrm{H}$ rats.

Responses were larger for the treated animals in all frequencies for the 2 month, 3 month and 4 month conditions. 
Figure 6.21 - Example of Flick ERG responses at different ages.

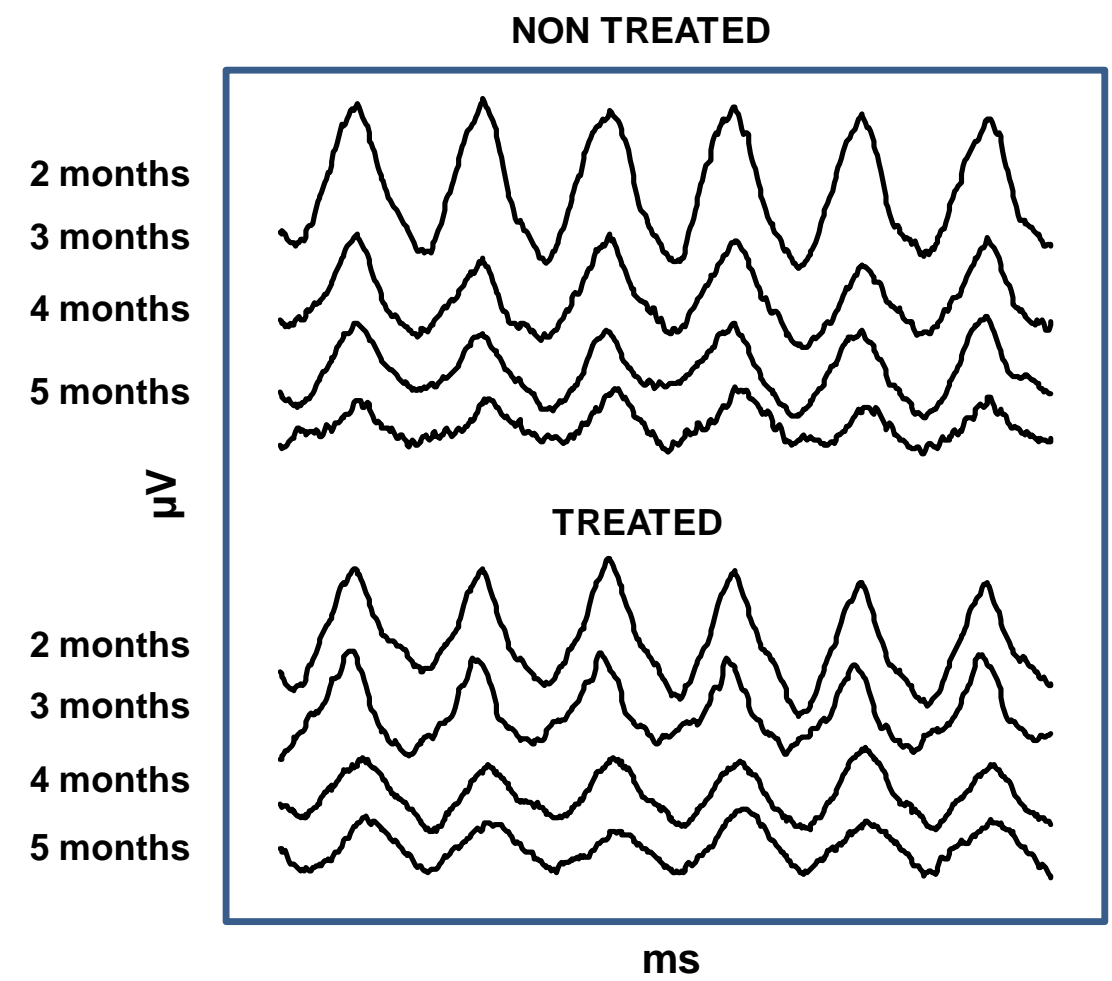

Example is from the intermittent response at $12 \mathrm{~Hz}$ frequency of non-treated and Ce6 treated rats at ages of 2, 3, 4 and 5 months old. Each line represents the data collected of only one rat (not average of all rats).

SOURCE: Balen, 2012

In Figure 6.22 the amplitudes of the $1^{\text {st }}$ harmonic measured at 12 and $30 \mathrm{~Hz}$ temporal frequencies are presented. 
Figure 6.22 - Average of $1^{\text {st }}$ harmonic response as a function of age at 12 and $30 \mathrm{~Hz}$ temporal frequencies.
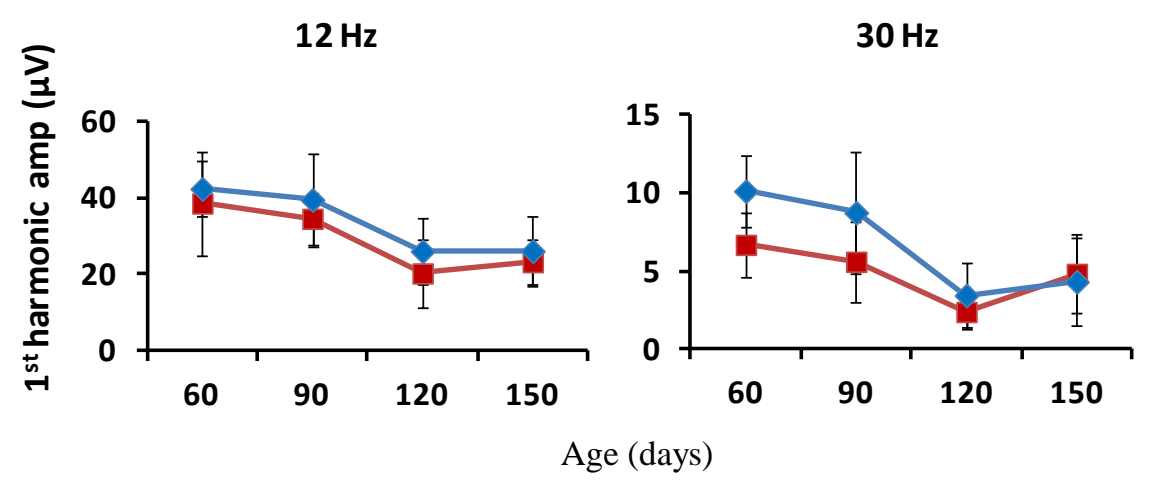

SOURCE: Balen, 2012

6.3.2 Treatment of S334ter rats with Ce6

Studies with the rat S334ter were carried out following the same protocol of $\mathrm{P} 23 \mathrm{H}$ rats.

\subsubsection{Morphological analysis}

Comparison between non-treated and treated retinal sections over the months revealed that $\mathrm{Ce} 6$ promoted a faster degeneration of the ONL (Figure 6.23). There was a reduction in the ONL thickness of Ce6 treated rats as compared to the control at P60, P90 and P150. 
Figure 6.23 - Comparison of S334ter rats treated and non-treated with Ce6 at P60, P90 and P150.
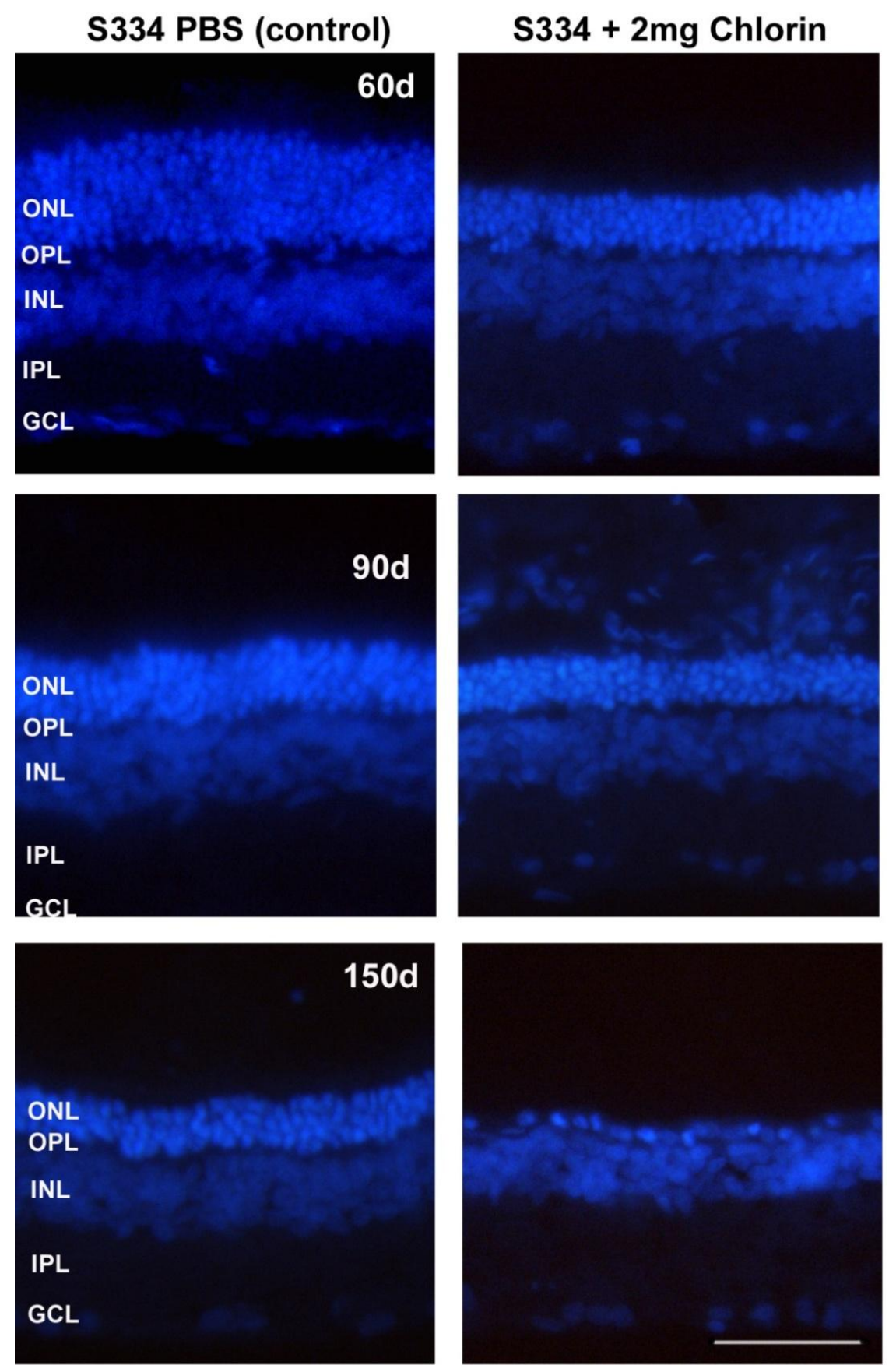

Retinal cross sections of S334ter rats non-treated (left side) and Ce6 treated (right side) stained with DAPI, a nuclear marker, to visualize the outer nuclear layer. A reduction in the ONL thickness can be observed after Ce6 treatment. At P150, a single layer of photoreceptor cell bodies can be seen in Ce6 treated retina. ONL, outer nuclear layer; OPL, outer plexiform layer; INL, inner nuclear layer; IPL, inner plexiform layer; GCL, ganglion cell layer. Scale bar: 50 $\mu \mathrm{m}$. 
SOURCE: Balen, 2012

The average ONL thickness values are shown in Figure 6.24 as a function of age for nontreated and $\mathrm{Ce} 6$ treated rats. Measurements of ONL thickness show that Ce6 treated S334ter rats retinas were more degenerated than non-treated rats. The effect occurs already at the early stages of treatment, P60, and continues through the period analyzed, P90 and P150. A plot of the ONL thickness at each area of the retina is shown in Figure 6.25.

Figure 6.24 - Mean ONL thickness of the Ce6 treated and non-treated S334ter rats.

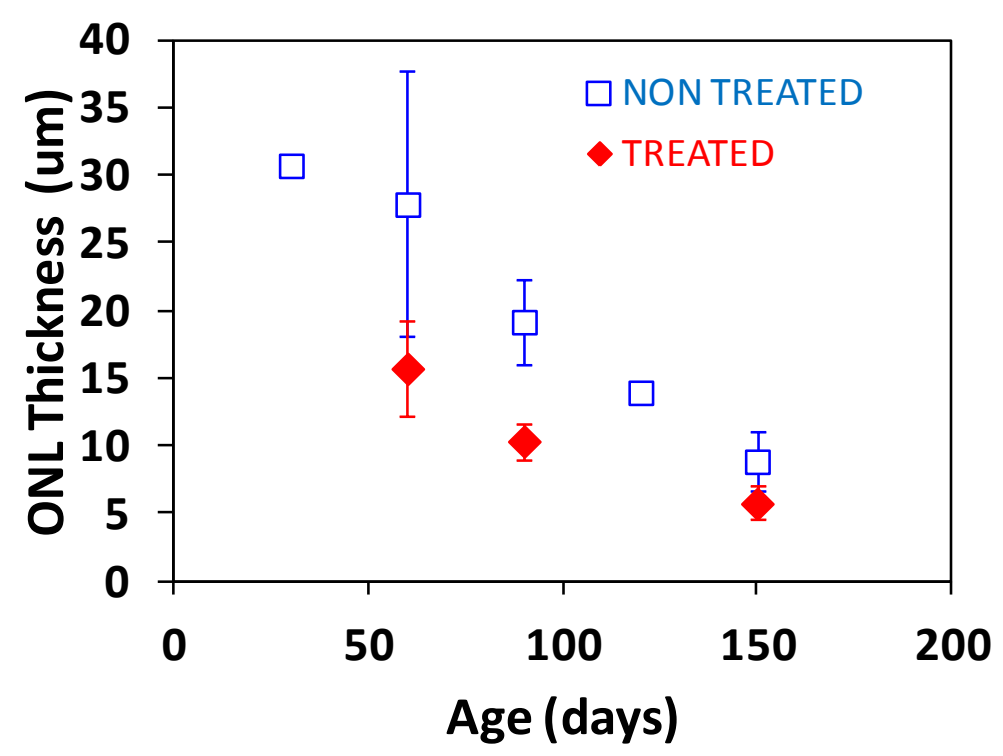

Values shown are an average of 3 measurements at each area. At P60, Ce6 treated rats have shown to have a retinal section half of the size of that of non-treated $\mathrm{P} 23 \mathrm{H}$ rats. The deterioration of the retina continued to be linear over the months, as seen by measurements at P90 and P150, for both groups. Ce6 treated rats had a larger loss of photoreceptor cell layers at the end of the measurement period. Number of animals (n) of non-treated rats at different age: 30 (3); 60 (2); 90(4); 120 (5) and 150 (3), and treated rats: 60 (3); 90 (3) and 150 (4).

SOURCE: Balen, 2012 
Figure 6.25 - Measurements of ONL thickness plotted as a function of the distance from the optical nerve head.

\section{S334ter non-treated}

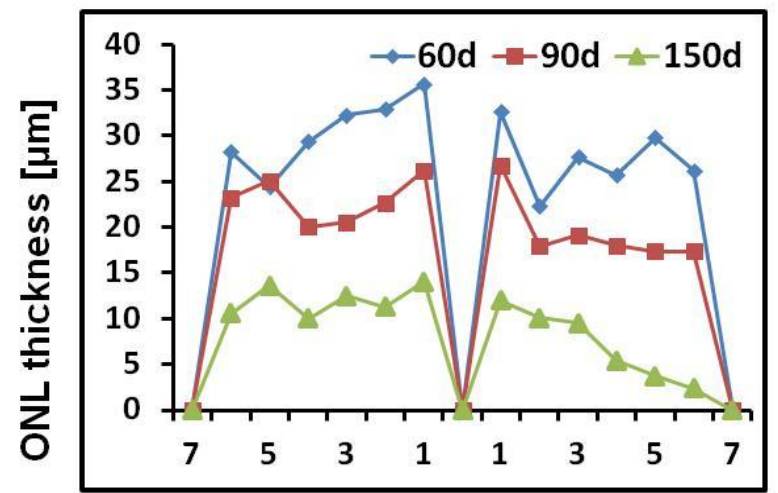

Distance from optical nerve
S334ter treated

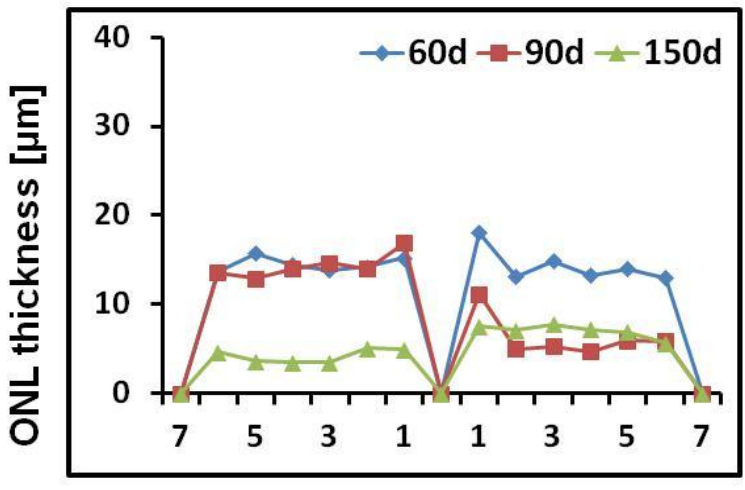

Distance from optical nerve

Left side of the optical nerve: dorsal side of eye. Right side of the optical nerve: ventral part of the eye. Values are an average of 3 measurements for each area. Number of animals (n) of non-treated rats at different age: 60 (2); 90 (4); and 150 (3), and treated rats: 60 (3); 90 (3) and 150 (4).

SOURCE: Balen, 2012

\subsubsection{ERG analysis of S334ter rats after treatment with Ce6}

To evaluate the effect of $\mathrm{Ce} 6$ on the functional activity of the S334ter rats, the ERG responses, scotopic and photophic, were recorded in both vehicle and Ce6 treated animals at different ages. Figure 6.26 illustrates the ERG responses obtained for both non-treated and treated groups at different ages. Detailed results of a- and b-waves, photopic and flicker measurements are shown in Figures 6.27 and 6.28. 
Figure 6.26 - Example of one rat response of ERGs total field (signal in $\mu \mathrm{V}$ ) of non-treated versus Ce6 treat animals $(2 \mathrm{mg} / \mathrm{Kg})$.

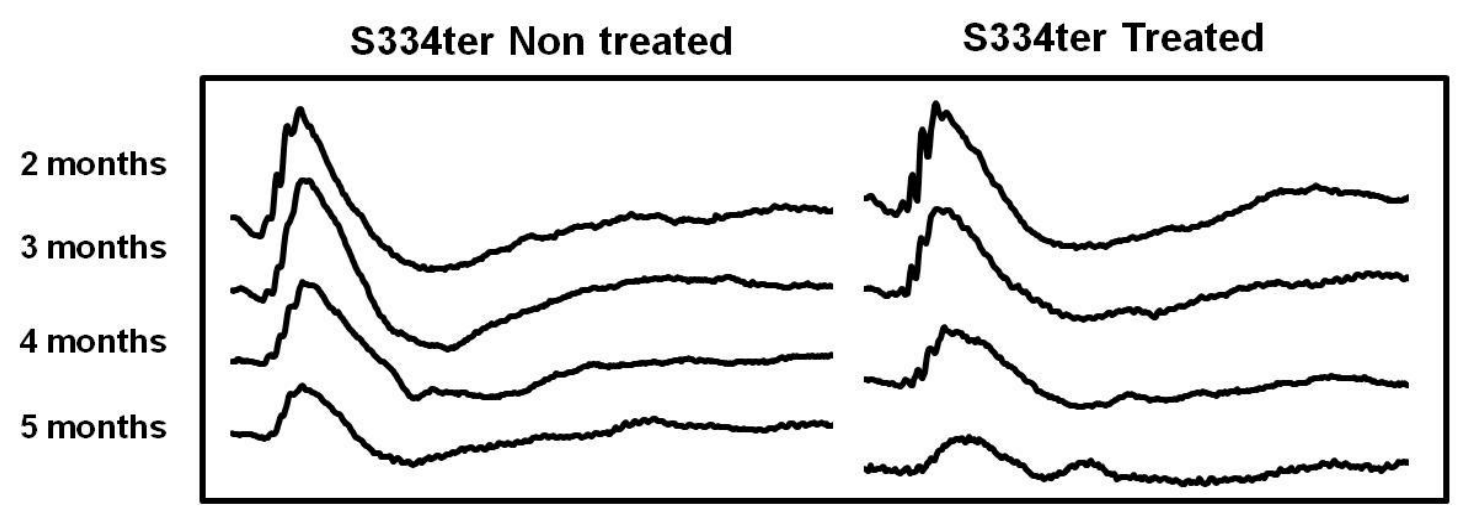

Treatment consisted of intraperitoneal injections every second day, starting at P20. ERG data was collected at P60, P90, P120 and P150. The data, for illustration, is the response of only one rat.

SOURCE: Balen, 2012

\section{a-wave:}

The scotopic a-wave amplitude response as a function of light intensity or age at the maximum intensity measured is shown in Figures 6.27 and 6.28, respectively. Figure 6.27 shows the overall ERG response at different light intensities. S334ter non-treated rats show a higher awave response at P60. For all other ages, a-wave measurements were similar between the two groups of rats. A large variation among the rats at each group was also observed, yielding a large standard deviation. On Ce6 treated S334ter rats, a-wave amplitudes of P60 and P90 rats were similar, which were different from that observed for P120 and P150 days, resulting in noisier results. 
Figure 6.27 - ERG amplitude response to different filters in S334ter rat.

\section{Scotopic ERG - Amplitude of a-wave}
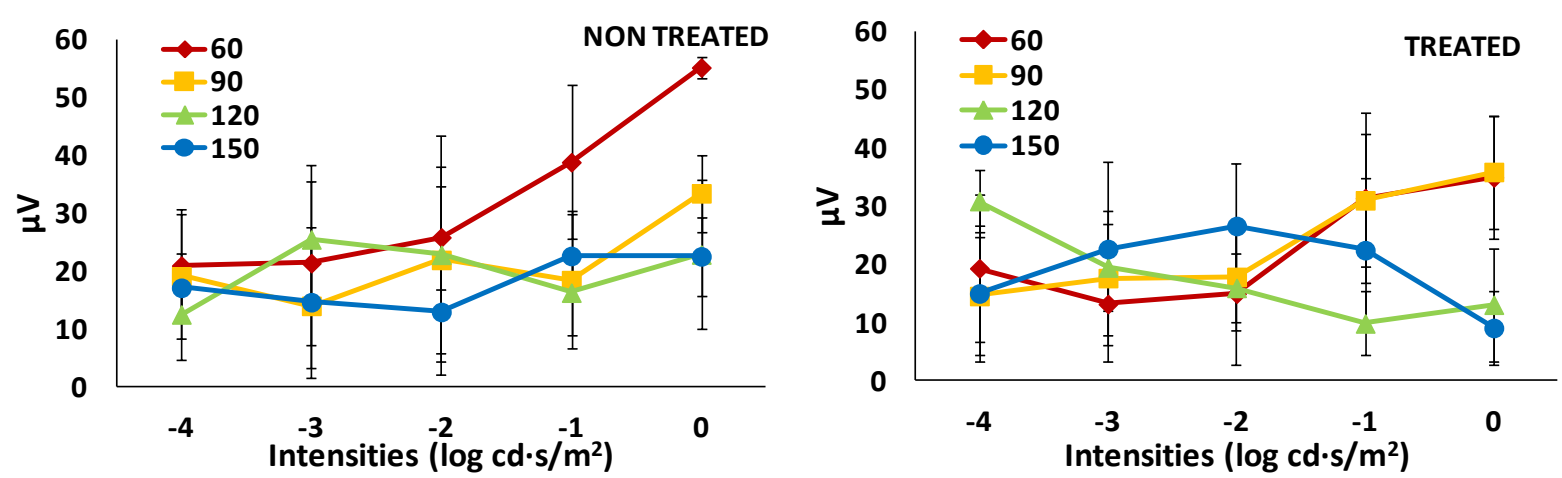

The average with standard deviation of the a-wave amplitude of the Ce6 treated and non treated S334ter rats are plotted. The number of animals (n) of non-treated rats at different age: 60 (5); 90 (5); 120 (4) and 150 (3). And treated rats: $60(8) ; 90(8) ; 120(4)$ and $150(4)$.

SOURCE: Balen, 2012

Amplitude responses of the treated and non-treated S334ter rats as a function of age at the maximum intensity was measured (Figure 6.28). Non-treated rats have shown larger amplitude than treated ones at P60, P120 and P150, whereas at P90 they appeared to be similar. Comparison of each group separately has shown that the non-treated a-wave response was larger than the treated group. The total percentage decreased from P60 to P150 animals, and it was calculated to be approximately $59 \pm 23 \%$ and $77 \pm 15 \%$ for non-treated and treated, respectively. 
Figure 6.28 - Average of a-wave amplitude (maximum scotopic response: $30 \mathrm{cd.s} / \mathrm{m}^{2}$ ).

\section{Scotopic ERG - Amplitude of a-wave}

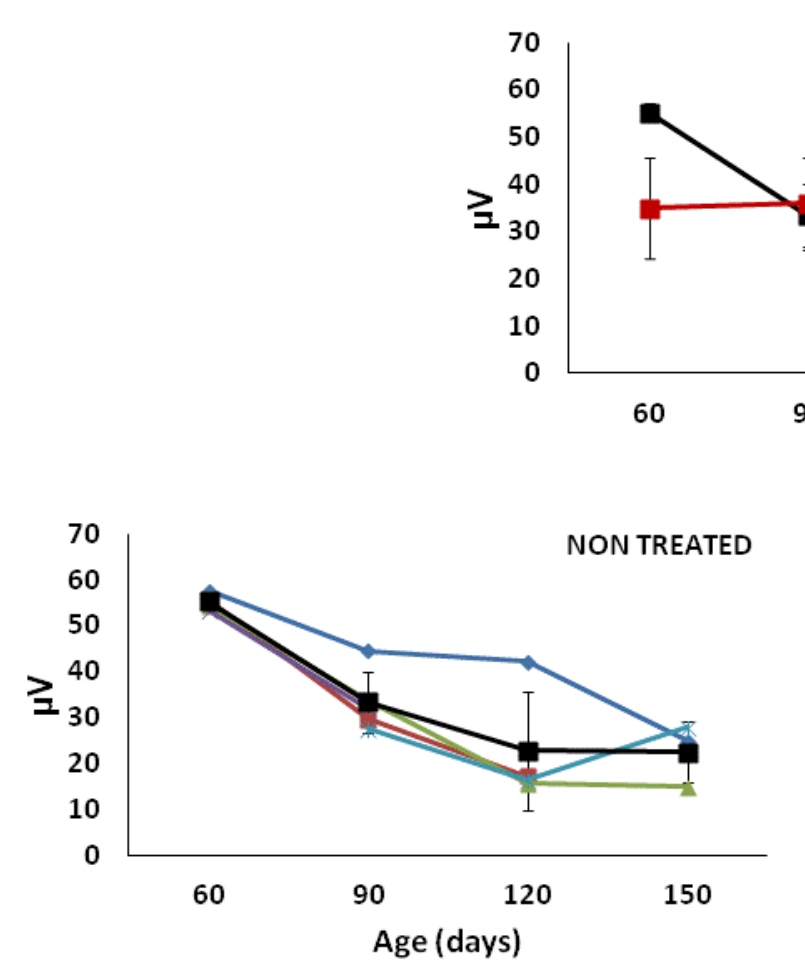

NON TREATED

TREATED

(A) Average with standard deviation of a-wave amplitude for treated and non-treated animals. Distribution of each individual and average of the group (black line) for (B) non-treated and (C) treated S334ter rats. Number of animals (n) of non-treated rats at different age: 60 (5); 90 (5); 120 (4) and 150 (3). And treated rats: 60 (8); 90 (8); 120 (4) and 150 (4).

SOURCE: Balen, 2012

The scotopic ERG latency of S334ter non-treated and treated rats was also recorded. The results are shown in Figure 6.29. Non-treated and treated rats presented around the same latency response at P60 and P90. Ce6 treated rats show a decreased response at P120 and P150, while non-treated rats display a nearly constant response over the time-range studied. 
Figure 6.29 - Average of a-wave latency (maximum scotopic response: $30 \mathrm{cd.s} / \mathrm{m}^{2}$ ).

\section{Scotopic ERG - Latency of a-wave}

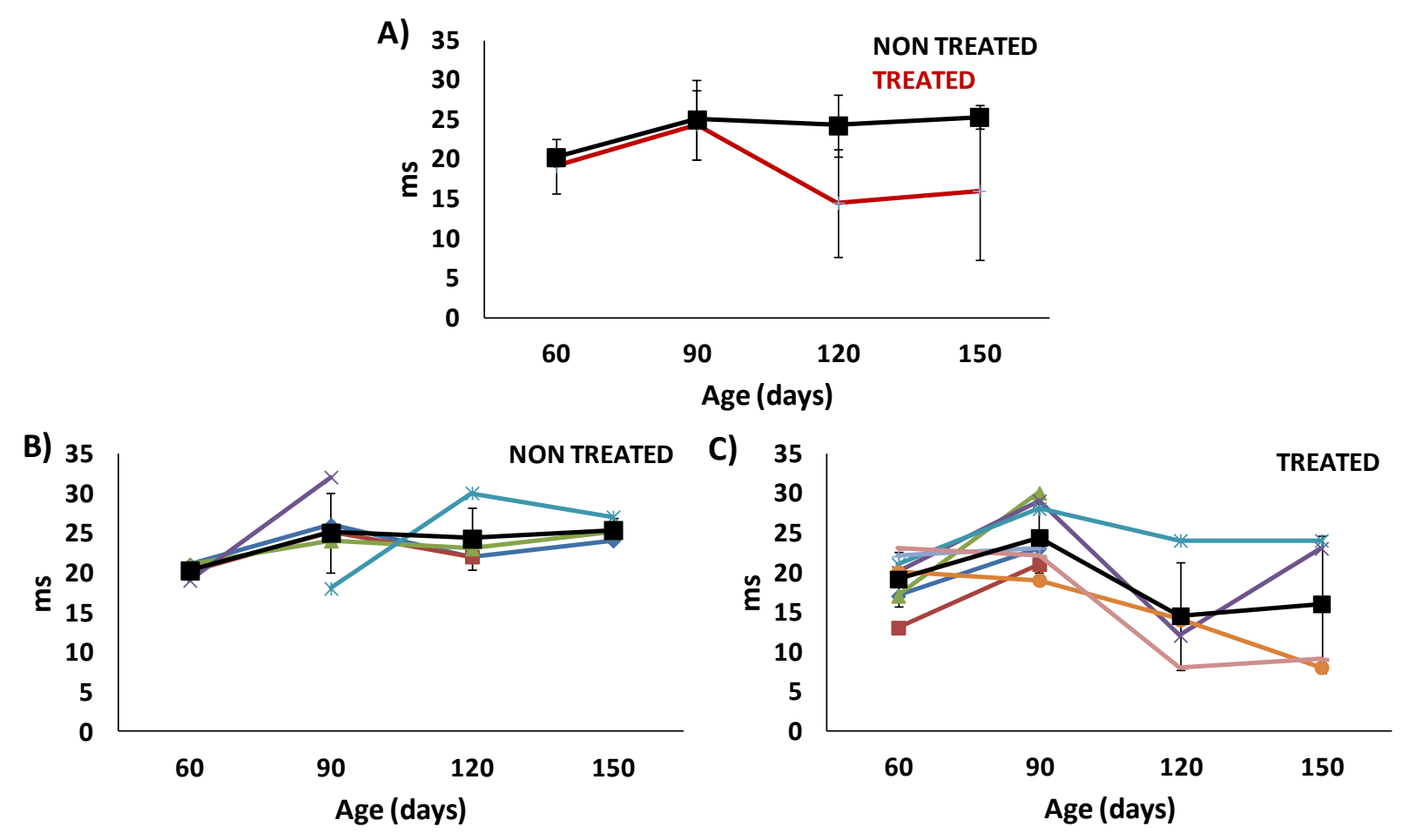

(A) Average with standard deviation of a-wave latency for treated and non-treated animals. Distribution of each individual and average of the group (black line) for (B) non-treated and (C) treated S334ter rats. Number of animals (n) of non-treated rats at different age in days: 60 (5); 90 (5); 120 (4) and 150 (3). And treated rats: 60 (8); 90 (8); $120(4)$ and $150(4)$.

SOURCE: Balen, 2012

\section{b-wave:}

Figure 6.30 shows the average of the b-wave amplitude response in all rats using different filters (see Chapter 2). A linear decrease of b-wave ERG response for all-non treated and treated rats was observed. Treated rats had a much smaller b-wave than that observed for non-treated animals. 
Figure 6.30 - ERG amplitude response as function of different filters in S334ter rats.

\section{Scotopic ERG - Amplitude of b-wave}
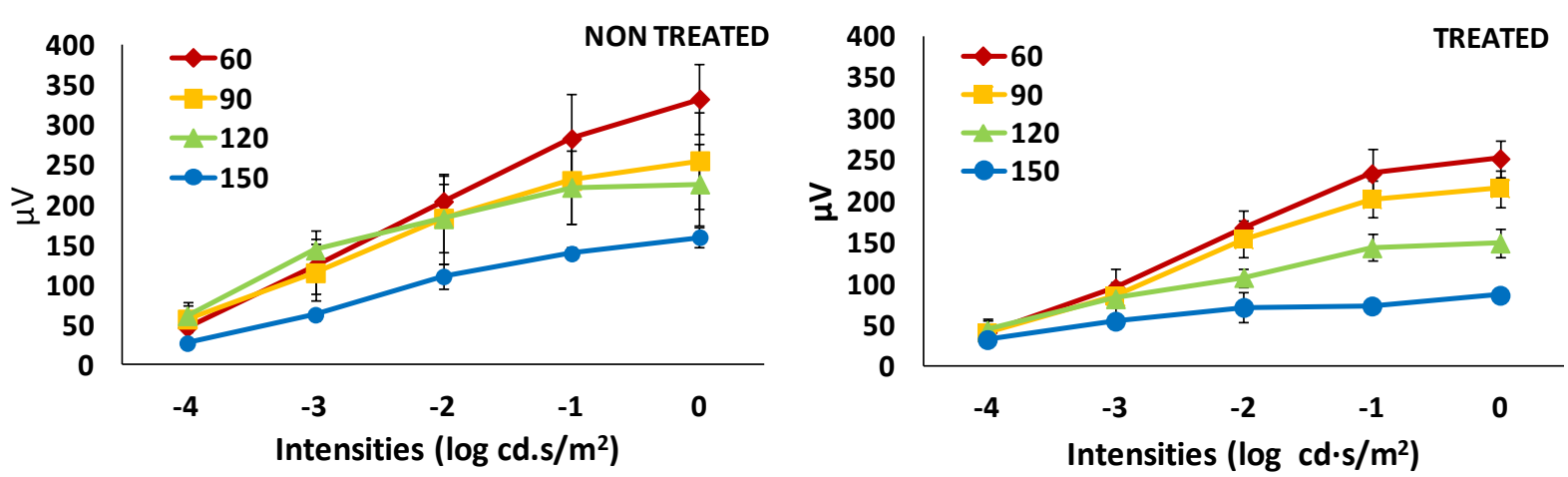

Figure shows the average with standard deviation of the b-wave amplitude of the Ce6 treated and non-treated rats. Number of animals (n) of non-treated rats at different age in days: 60 (5); 90 (5); 120 (4) and 150 (3). And treated rats: 60 (8); 90 (8); 120 (4) and $150(4)$.

SOURCE: Balen, 2012

A comparison of the average b-wave response amplitudes of the non-treated and treated rats for the maximum intensity of light measured as a function of age is shown in Figure 6.31. The average b-wave response was higher at all measured ages for non-treated as compared to Ce6-treated rats. The total decrease of the b-wave amplitude from the first time point, P60, to the last collected data point, P150, was $40 \pm 18 \%$ and $67 \pm 15 \%$ for non-treated and treated rats, respectively. 
Figure 6.31 - ERG amplitude response in S334ter rats as a function of age.

\section{Scotopic ERG - Amplitude of b-wave}
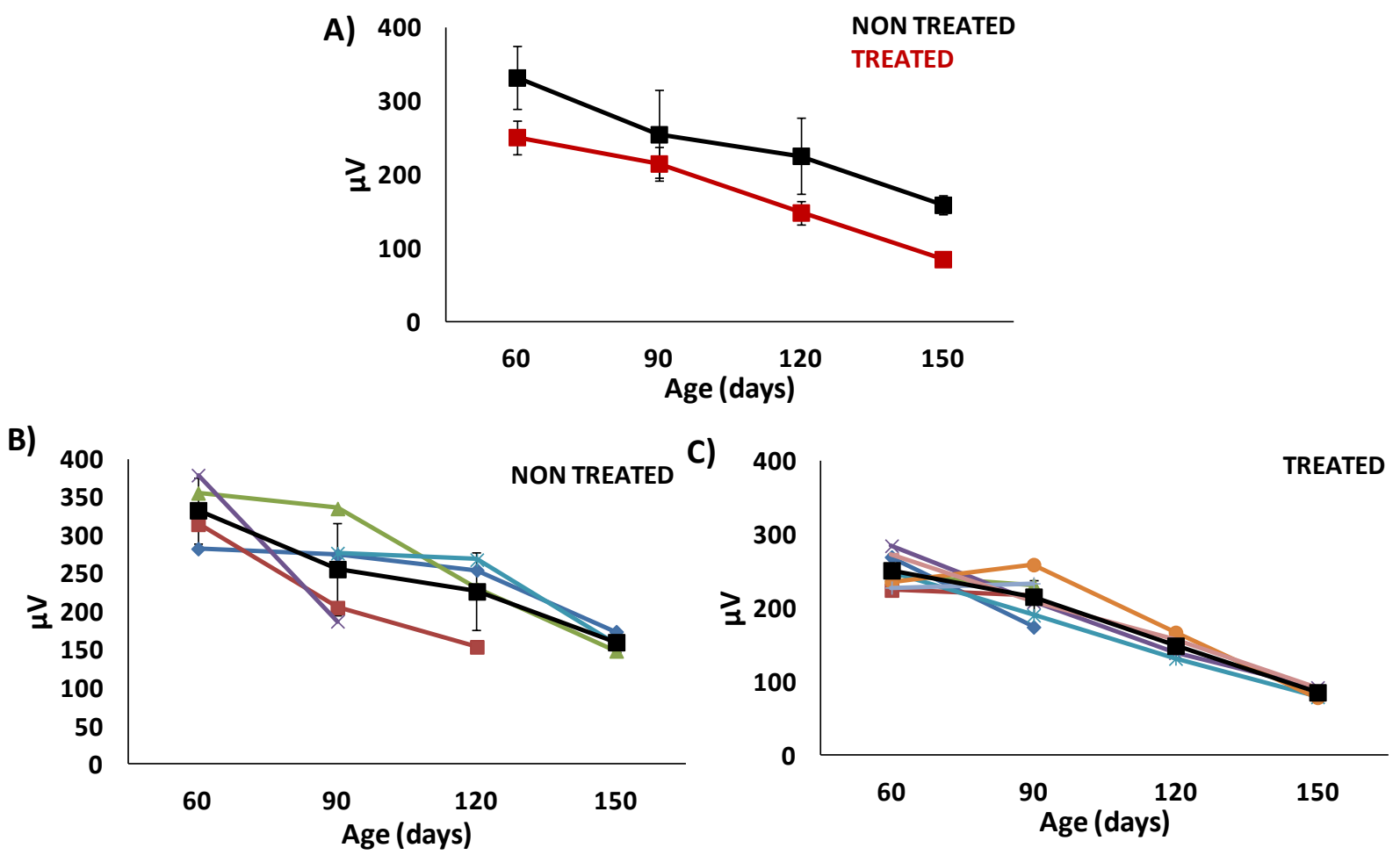

Average with standard deviation of b-wave amplitude of the ce6 treated and non-treaded S334ter rats (A) and distribution of $b$-wave amplitude of each rat non-treated (B) and treated (C). Colored lines represent each individual tested. Black line is the average of the group (B and C). Number of animals (n) of non-treated rats at different age in days: 60 (5); 90 (5); 120 (4) and 150 (3). And treated rats: 60 (8); 90 (8); 120 (4) and 150 (4).

SOURCE: Balen, 2012

The ERG latency of the b-wave response for both non-treated and treated S334ter rats at scotopic conditions was measured and the results are shown in Figure 6.32. No variability in the response at different ages or between treated and non-treated rats were found. 
Figure 6.32 - ERG latency of b-wave response in S334ter rats.

\section{Scotopic ERG - Latency of b-wave}

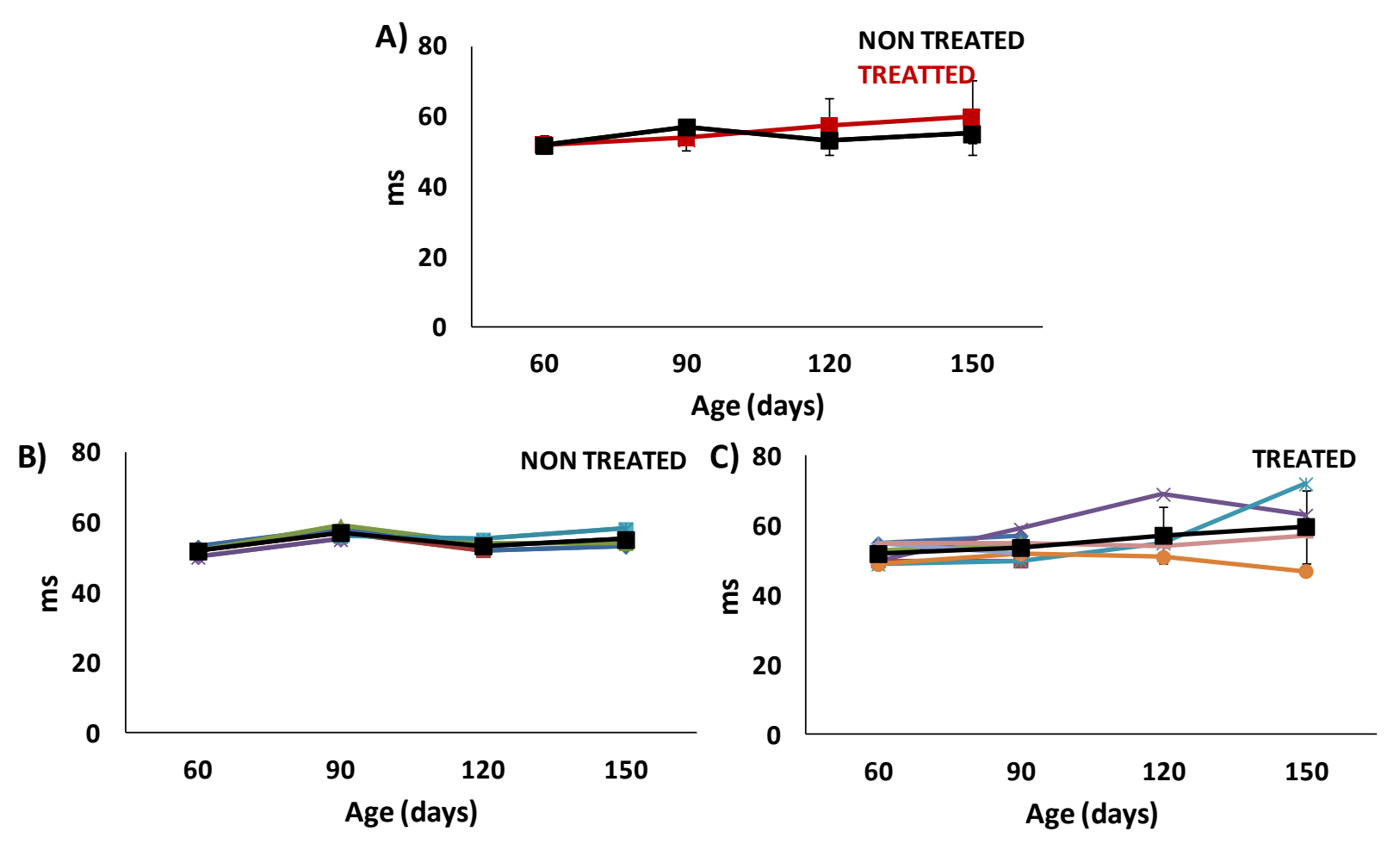

Average with standard deviation of b-wave latency of the Ce6 treated and non-treated S334ter (A), response of all rats tested together, color lines, with averages, black lines are plotted for non-treated (B) and Ce6 treated rats (C). Number of animals (n) of non-treated rats at different age in days: 60 (5); 90 (5); 120 (4) and 150 (3). And treated rats: 60 (8); 90 (8); 120 (4) and 150 (4).

SOURCE: Balen, 2012

\section{Photopic ERG response:}

Under photopic conditions, ERG responses of Ce6-treated S334ter rats in comparison to non-treated animals showed that they were larger at P60 and P90 (Figure 6.33). At P120, nontreated rats had a slightly higher average response, although at P150 average responses were similar. 
Figure 6.33 - Photopic ERG response in S334ter rat.

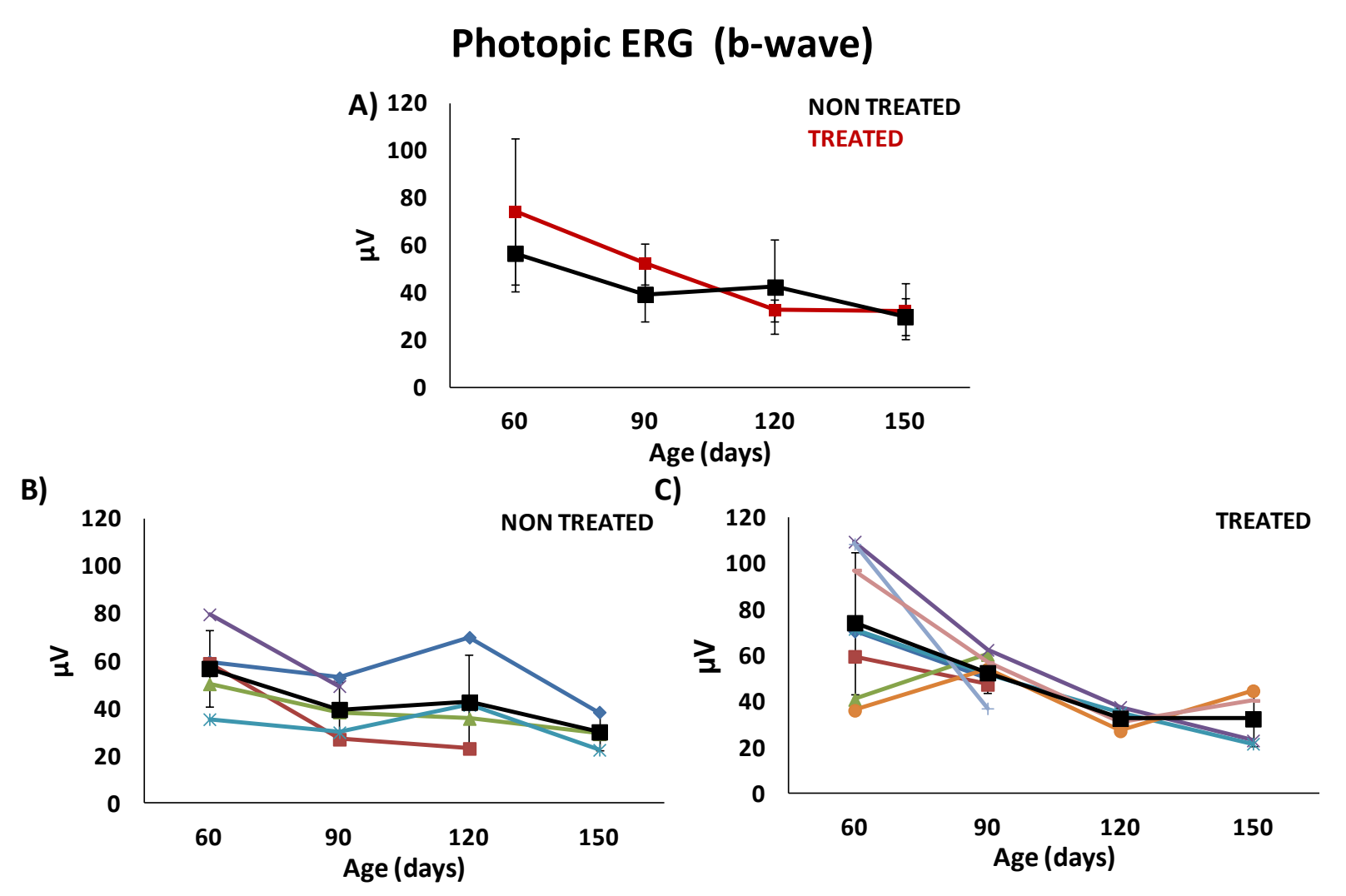

Average of the ERG photopic response of the Ce6 treated and non-treated S334ter rats (A) average and total response of non-treated rats (B) and Ce6 treated (C). Colored lines represent each individual tested. Black line is the average of the group.

SOURCE: Balen, 2012

\section{Flicker:}

The Flicker response was recorded in order to assess cone activity on S334ter rats, as shown in Figure 6.34. 
Figure 6.34 - Example of Flicker ERG responses at different ages.

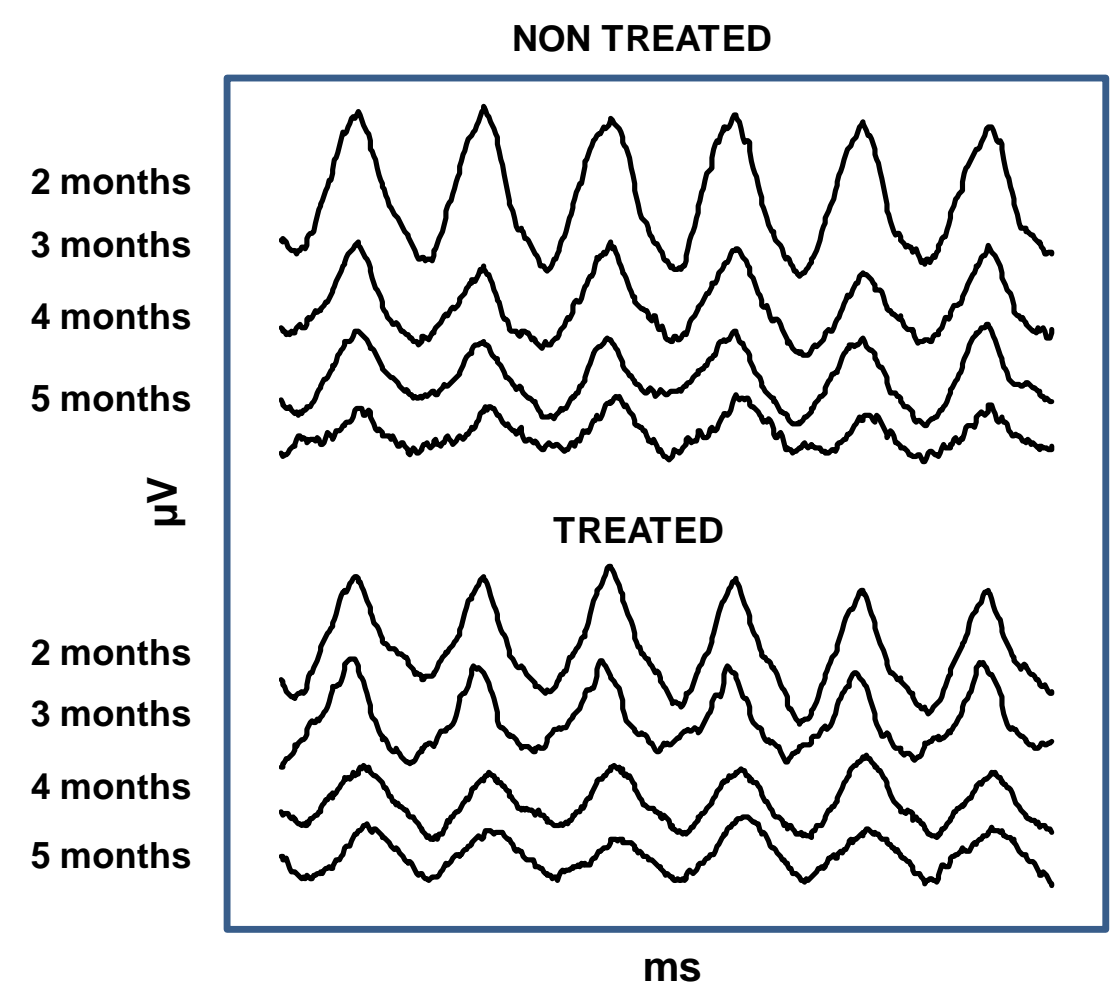

Example is from the intermittent response at $12 \mathrm{~Hz}$ frequency of non-treated and treated rats at ages of 2, 3, 4 and 5 month old animals. The data, for illustration, is the response of only one rat.

SOURCE: Balen, 2012

Figure 6.35 presents the amplitudes of the $1^{\text {st }}$ harmonics after Fourier transform of the data shown in Figure 6.34 in response to 12 and $30 \mathrm{~Hz}$ frequencies for Ce6 treated and nontreated rats. Amplitudes of the 1st harmonics diminished according to the frequency of stimulation. The maximum amplitude of the 1 st harmonic was $40 \mu \mathrm{V}$ at $12 \mathrm{~Hz}$, and around $6 \mu \mathrm{V}$ at $30 \mathrm{~Hz}$. At P60 and P120 the amplitude of both, non-treated and Ce6-treated groups presented a similar average value. At P90 and 150, Ce6-treated rats showed larger amplitude values. 
Figure 6.35 - Average of $1^{\text {st }}$ harmonic response as function of age at 12 and $30 \mathrm{~Hz}$ temporal frequencies.
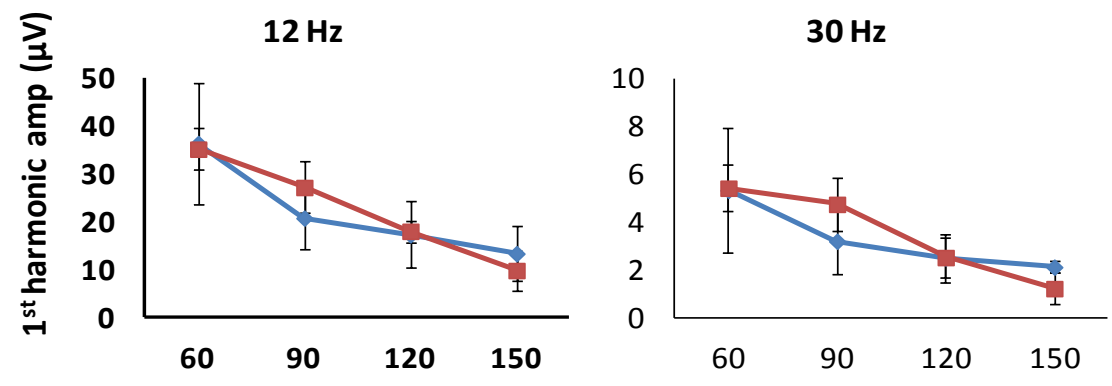

Age (days)

SOURCE: Balen, 2012

\subsection{DISCUSSION}

In this chapter the goal was to investigate whether Ce6 had an effect on the prevention of retinal degeneration in $\mathrm{RP}$ rat models $\mathrm{P} 23 \mathrm{H}$ and $\mathrm{S} 334$ ter. We found that $\mathrm{Ce} 6$ partially reduced hereditary retinal damage in the $\mathrm{P} 23 \mathrm{H}-3$ rat, whereas appears to accelerate retinal damage in the S334ter-4 rats.

The transgenic $\mathrm{P} 23 \mathrm{H}$ rat was engineered to mimic a mutation found in humans, which is the most common mutation observed in RP patients (Dryja et al., 1990). P23H rats, although initially presenting a normal cone function, develop a progressive rod dysfunction, the pattern being at some extent very similar to the pattern clinically found in humans (Pinilla et al., 2005). There are different lines of $\mathrm{P} 23 \mathrm{H}$ rat available and line 3 was chosen due to its slow retinal degeneration rate, which is the most similar to that found in humans. This choice was also important because the slow rate of degeneration allows for assessment of retinal progression at early ages. For $\mathrm{P} 23 \mathrm{H}$, degeneration is already measurable after only 120 days, but also as late as 360 days as these rats retain vision for a long time (LaVail and Battelle, 1975).

At first, to test dose dependency, Ce6 was intraperitoneal injected every second day from P35 to P60 at different concentrations: 2, 10 and $20 \mathrm{mg} / \mathrm{kg}$. Although the outer nuclear layer was more preserved in Ce6-treated rats, the effect was not dose-dependent, and for this reason the lowest dose $(2 \mathrm{mg} / \mathrm{Kg})$ was chosen for further Ce6 experiments. Apparently, at least using the present protocol, DMTU (500 mg/Kg) used in previous studies (Organisciak et al., 1992, 2003) was more efficient in preserving retinal structure. The next step was to perform a long-time 
treatment with $\mathrm{Ce} 6$ in the $\mathrm{P} 23 \mathrm{H}$ and S334ter rats. A systemic treatment started when rats were 20 days old and followed every other day until they reached 120 days. Administration of Ce6 in $\mathrm{P} 23 \mathrm{H}$ rats resulted in a positive effect on retinal cell layer preservation. Morphological analysis using immunostaining techniques showed a preservation of the photoreceptors. In the comparison of rats at 90,120 and 150 days of age, the Ce6 group shows a larger number of outer nuclear layer rows while the retina is normally more deteriorated in the control rats. Yet, at 60 days old the outer nuclear layer of the non-treated rats seemed to have more rows than those that were treated. Thickness measurements of the outer nuclear layer show that deterioration did not increase between P60 and P90 for Ce6 rats, while non-treated deteriorate much over for this timerange. Comparison at $\mathrm{P} 150$, for both groups, demonstrated that at this age the degeneration pattern was the same and that early treatment did not mediate a difference in response. No rats were sacrificed at P120, the date at which the treatment was stopped. It can be inferred from the data collected until P90 that Ce6 treatment was efficient during the administration period. As expected, after it was stopped at P120, the degeneration followed the same pattern as that of the control group.

The preservation was also evaluated by ERG measurements. Relatively larger amplitudes at P60 for non-treated rats as compared to that of treated rats were observed, in agreement with the preservation of the thickness of the outer nuclear layer. However, since ERG measurements were not performed prior the treatment started at P20, and ERG measurements present a variation among different rats, a value for P20 cannot be estimated based on a $3^{\text {rd }}$ group of rats for example. Latencies of a- and b-wave were also analyzed, but no differences were observed. Photopic and flicker response also suggest a better functional response of Ce6 treated rats. The photopic response was the equivalent for both groups at P60 and P150, while at P90 and P120 Ce6 treated rats showed a higher average response. Flicker ERG was recorded for 12, 18, 24 and $30 \mathrm{~Hz}$, in overall all of the responses of $1^{\text {st }}, 2^{\text {nd }}$ and $3^{\text {rd }}$ harmonics were larger for Ce6 treated at P60, P90 and 120. At P150, the results were similar for both groups.

In addition to the functional, morphological and photoreceptor layer preservation, details on the connectivity between photoreceptors and their postsynaptic neurons were exploited by looking at the rod bipolar cell morphology using the antibody against protein kinase $\mathrm{C}$ (PKC). In control non-treated rats, rod bipolar cells showed an apparent retraction of their dendrites and general disorganization of cell bodies at P90. By contrast, Ce6-treated $\mathrm{P} 23 \mathrm{H}$ rats presented a 
higher density of rod bipolar cells bodies and dendritic terminals and better organization. The loss of bipolar cell dendrites was reduced. This result is in direct support of the observed preservation of the photoreceptors. Horizontal cells, which are located in the outermost INL and which make connections with rod and cone photoreceptors and the presynaptic terminals of rods and cones were also investigated using the antibodies against calbindin and synaptophysin (SYP), respectively. The retinal sectionals analysis was not conclusive.

Overall, $\mathrm{Ce} 6$ appears to exert a positive effect in the $\mathrm{P} 23 \mathrm{H}$ rat model. The results strongly suggest that the effect observed, although small, on the preservation of photoreceptors morphology and function is not only observed for the photoreceptors, rods and cones, but can also be extend to other cell types of the retina, promoting the prevention of secondary degeneration and remodeling of retinal circuitries.

In contrast, administration of $\mathrm{Ce} 6$ in the S334ter rats mediated an inverse response, accelerating the rate of photoreceptor degeneration. Heterozygous transgenic S334ter rats, line 4, develop slow photoreceptor cell death due to the mutant rhodopsin gene that presents a termination at residue 334 (Glybina et al., 2010). This termination occurs at the C-terminal side of rhodopsin, a site believed to contribute majorly to binding of Ce6 in WT rhodopsin (Yanamala, 2009).

Morphological analysis of S334ter rats non-treated and treated with Ce6 showed that rats submitted to treatment suffered a larger reduction of the photoreceptor layers as compared to non-treated rats. At P60 and P90, the outer nuclear layer of Ce6 treated rats were half of the size of that of non-treated rats. At P150, around three layers were still present in non-treated rats while only one was preserved in the Ce6 treated rats. Functional assessment was done by electrophysiology measurements. Lower amplitudes of both scotopic a- and b-waves were found in Ce6 treated in comparison to control animals. Normalized data showed that non-treated rats had no decrease on a-wave amplitude from P60 to P90, and P60 to P120, while they presented a $59 \pm 23 \%$ decrease at P150. Ce6-treated animals also do not show a decrease from P60 to P90. However from P60 to P120 and P150, the decrease was $66 \pm 28 \%$ and $77 \pm 15 \%$, respectively. The non-treated rats' $b$-wave amplitude decrease was calculated to be $11 \pm 33 \%, 16 \pm 36 \%$ and $40 \pm 18 \%$ from P60 to P90, P120 and P150, respectively. Ce6-treated presented a larger decrease at all months. 
The latency of the a-wave was the same at P60 and P90, while smaller for Ce6-treated rats at P120 and P150. But no changes were observed for b-wave latencies. A higher photopic ERG response was observed in Ce6-treated animals at P60 and P90, while similar responses were observed at P120 and P150. Similar results were also observed for the flicker ERG recordings, where a slightly larger response was observed at P60 and P90 for Ce6 treated rats and a similar response for both groups was observed at P120 and P150.

Studies have shown that the chlorophyll derivative Ce6 enhances the sensitivity of rhodopsin to red light in deep-sea fish rhodopsin (Douglas et al., 1999; Isayama et al., 2006). In both, rats and rabbits, when Ce6 was administered either orally or intravenously, it localizes to the retina and retinal pigment epithelium (Washington et al., 2007). Studies with animal models in vivo, such as salamander and mouse, have confirmed that Ce6 can effectively enhance vision (Washington et al., 2007). Mice administered with Ce6, showed a two-fold increase in ERG bwave amplitudes as a response to red and blue light. 


\subsection{CONCLUSIONS}

Here, the role of $\mathrm{Ce} 6$ on the protection of retinal damage in the RP rat models, $\mathrm{P} 23 \mathrm{H}$ and S334ter, was investigated. Ce6 partially reduces hereditary retinal damage in the $\mathrm{P} 23 \mathrm{H}$ rat as evidenced by the morphological analysis, where a preservation of the photoreceptor layer was observed and by the functional response improvement shown by ERG analysis. On the other hand, Ce6 accelerated retinal damage in the S334ter rats as suggested by the morphological and functional analysis where photoreceptor layer and ERG responses were much more deteriorated than control S334ter rats. The differences in the results obtained for the two rat types can be attributed to the nature of the mutation: $\mathrm{P} 23 \mathrm{H}$ has a point mutation at the $\mathrm{N}$-terminus portion of rhodopsin, the opposite side of that Ce6 is believed to bind. S334ter expresses an opsin gene with a termination codon at residue 334, resulting in a C-terminal truncated opsin protein which cannot be phosphorylated (Martinez-Navarrete et al., 2011), and may also interfere in Ce6 bind to the protein. Thus, we hypothesize that Ce6 can no longer bind to rhodopsin and can thus not stabilize rhodopsin, in contrast to the $\mathrm{P} 23 \mathrm{H}$ mutant. This hypothesis requires future experiments to validate. 


\section{CHAPTER 7: INTEGRATION OF IN VITRO, IN VIVO RAT MODEL AND PATIENT STUDIES IN RETINITIS PIGMENTOSA AND FUTURE STUDIES}

\subsection{SUMMARY}

In parallel to our molecular and in vivo model studies, our collaborator Alexander Iannaccone at the Retinal Degeneration \& Ophthalmic Genetics Service \& Lions Visual Function Diagnostic Laboratory, Hamilton Eye Institute, Department of Ophthalmology, and University of Tennessee Health Science Center, USA, conducted patient studies of P23H and N15S mutants. This provides us with the opportunity to compare in vitro, in vivo and patient data directly. Here, I therefore present a summary of the major findings of my work integrated with the patient studies. To this end, the manifestations, variability in severity and disease progression rates obtained from patients pertaining to families carrying either the $\mathrm{N} 15 \mathrm{~S}$ or $\mathrm{P} 23 \mathrm{H}$ mutation is compared with the in vitro biochemical properties of the corresponding mutant rhodopsin. The results are integrated into a comprehensive model for the role of rhodopsin as a target for small molecules in RP degeneration and rescue. Finally, the prospects for novel approaches to prevent and treat RP in animals and patients are discussed.

\subsection{INTRODUCTION}

Based on clinical studies, ADRP is divided into two classes - class A and B (Cideciyan et al., 1998). Class A is characterized by severe rod function abnormalities based on ERG and perimetry measurement, which results in consistent early night blindness while there is measurable cone-mediated activity even later in life. On the other hand, Class B shows measurable rod function (partial ERG preservation) as well as preserved cone function and is characterized by variable onset of night blindness (some asymptomatic). Class B is further divided into subclasses B1 and B2. B1 presents regional variation of retinal damage, while B2 indicates a diffuse disease condition with no regional predilection. Therefore, in class B mutants, cone loss is spatially and temporally related to rod loss and the rate of cone loss is slower than that of rods. 
Bridging the gap between the classifications of mutants based on disease phenotype in patients and the classifications based on in vitro biochemical properties of $R H O$ mutations, requires a detailed comparison between the clinical manifestations of the mutations and the molecular properties of the proteins. Many rhodopsin mutations have been studied in vitro and are classified according to its in vitro behavior (Kaushal and Khorana, 1994; Sung et al., 1991, 1993). In vitro classification of ADRP mutants has been carried out based on their molecular phenotype, with three classes (Kaushal and Khorana, 1994): Class I, II and III. Class I resembles WT rhodopsin where the mutants fold correctly, bind the 11-cis retinal chromophore normally, but are inefficient in the inactivation of transducin, the cognate $G$ protein complex. Class II mutants do not traffic out of the endoplasmic reticulum and do not bind 11-cis retinal. Class III mutants are expressed at low levels, poorly form rhodopsin chromophore and are also retained in the endoplasmic reticulum, presenting high mannose glycosylation.

Previously, several class B ADRP patients, carrying mutations in different domains of rhodopsin, namely in the extracellular domain, in the second intradiscal loop (P180A and G188R), and in the cytoplasmic domain, at the end of helix III (R135L and R135W) were studied (Iannaccone et al., 2006). The degrees of severity and progression rates in patients correlated with the computationally predicted and experimentally confirmed molecular properties of the respective rhodopsin protein (Iannaccone et al., 2006).

In this thesis, I investigated two mutants located at the same region of rhodopsin $(\mathrm{N}-$ terminus in the extracellular domain). They are both grouped in the same class, based on clinical phenotype and molecular properties. This provided an opportunity to test the hypothesis that clinical phenotype and molecular properties are related at a finer grained level. To this end, an indepth comparison of the molecular data with the clinical characteristics studied by our collaborator is provided in this chapter.

The in vitro data described in this thesis showed that the mutant rhodopsin proteins N15S and $\mathrm{P} 23 \mathrm{H}$ are structurally impaired, but present different characteristics and severity of impairment. There is to date no rat or mouse model of N15S, so a comparison in an in vivo animal model is currently not possible. We are therefore fortunate to have access to in vivo patient data instead through our collaborator, Alex Iannacone, University of Tennessee. His in vivo patient data, summarized in this chapter, below, confirms the phenotype observed in vitro. Even though both mutations belong to the same class, the investigated patients presented 
remarkable differences. $\mathrm{P} 23 \mathrm{H}$ affected patient show a greater disease severity than the N15S patient. This supports the conclusion that the instability of the rhodopsin protein structure is linked to disease phenotype and severity. Thus, it should be possible to lessen the disease burden of patients with strategies that stabilize the rhodopsin structure. In order to improve the conditions of RP affected patients, new strategies for treatment of RP are being explored, and are at different stages of trial. Here, the use of different ligands was tested, as a new approach for the stabilization of the structure of rhodopsin. We first demonstrated the success of this strategy in vitro. Since the results in vivo were promising, especially for $\mathrm{Ce} 6$ alone and in conjunction with

$\mathrm{Zn}^{2+}$, it was also tested if $\mathrm{Ce} 6$ would also help to stabilize the mutant protein in vivo. While we cannot test binding and stabilization effects on rhodopsin in vivo, we can use the end product prevention of retinal degeneration as indirect evidence for a success in treatment. To this end, two studies were performed. First, a light-degeneration model, and secondly two inherited RP rat models. Ce6 did show some (albeit weak) evidence for a prevention of light-induced retinal degeneration, but did have a clearly positive effect in one of the models, P23H. Interestingly, a negative effect was observed in the other rat model, S334ter. These effects are likely attributable to the type of the mutation and its localization.

\subsubsection{Summary of patient data carrying $\mathrm{P} 23 \mathrm{H}$ and N15S mutations}

Clinical studies were performed by Alexander Iannaccone are briefly summarized here. Two individuals of each family were investigated, each of them presenting one of the two RP mutations studied, P23H and N15S. The genealogies of the families are shown in Figure 7.1. 
Figure 7.1 - Pedigrees of the two families investigated in this study.
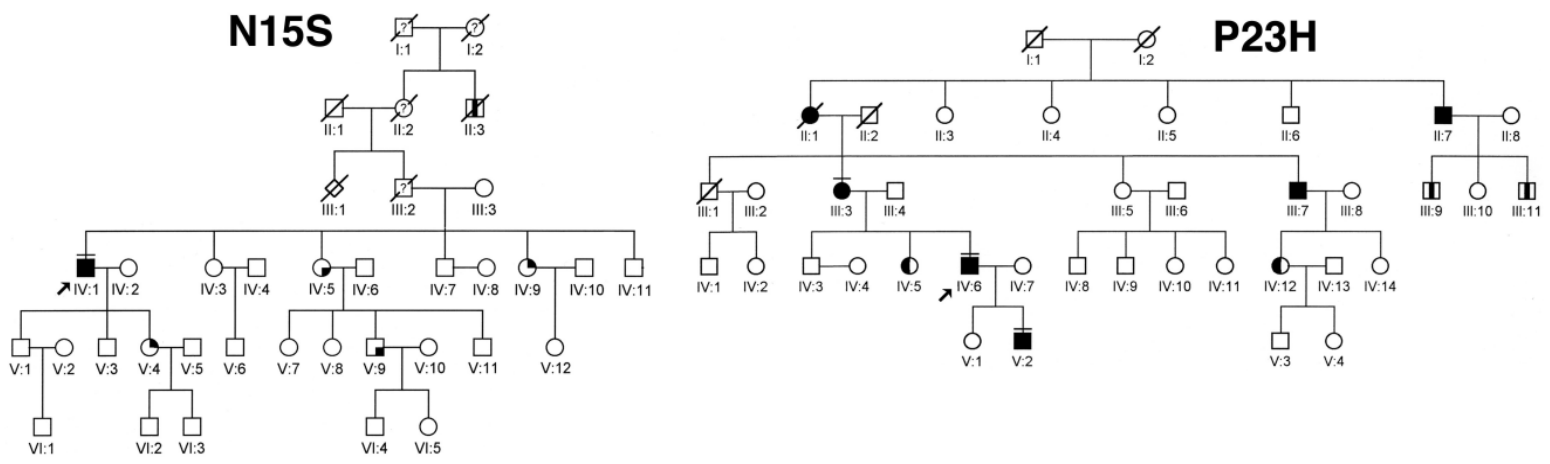

Probands from each family are showed by the arrow sign. Horizontal bar on top of the symbol correspond to subjects that were examined. Filled symbols identify affected individuals.

SOURCE: Not published. With permission.

Clinical studies included full-field flash ERG testing, Goldmann visual fields (GVFs), monochromatic automated perimetry (MAP) and dark adaptometry (DAPT). In all patients, the onset of night blindness was delayed ( $>30$ years old) and visual acuity was $\geq 20 / 30$, but disease expression varied in severity across these $R H O$ changes. Thus, both $\mathrm{N}$-terminal mutations studied here are associated with a Class B phenotype.

The N15S mutation was associated with mild and sharply demarcated inferior retinal disease. Where in the inferior fields, both rod sensitivity loss (RSL) and cone sensitivity loss (CSL) were minimal at age 62, but rod dark adaptation was markedly and unevenly delayed across the entire visual field even after exposure to moderate amounts of ambient light. At age 66, Goldmann visual fields (GVFs) were virtually unchanged, CSL had slightly increased around fixation, and RSL had increased pericentrally. At age 62, rod, mixed, and cone ERGs of the N15S patient were reduced only about $80 \%, 60 \%$ and $75 \%$ of the lowest limits of normal, respectively, configuring a cone>rod pattern of retinal dysfunction. At age 66, rod and mixed ERGs had declined to about $50 \%$ of the normal lowest limits observed, whereas cone ERGs were unchanged. Formal DAPT testing at age 66 showed markedly delayed recovery kinetics of both rods and cones. 
Figure 7.2 - Representative ISCEV-standard ERG response.

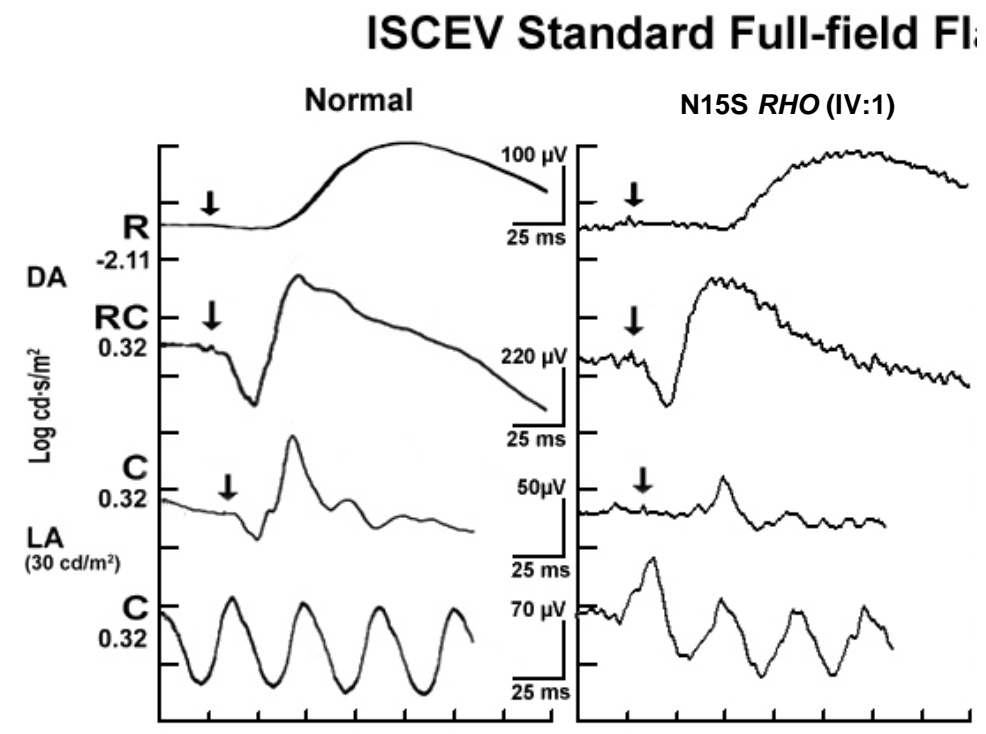

Full-field flash ERG testing of a N15S patient.

SOURCE: Not published. With permission.

Similar to the N15S mutation, $\mathrm{P} 23 \mathrm{H}$ also had regional (inferior and nasal) retinal disease predilection. This Class-B1 pattern was observed at age 12 (V:2) clinically and at the GVF as well as at the MAP level. However, unlike the N15S mutation, by age 41 (IV:6) the disease pattern transitioned to a widespread RSL>CSL (Class-B2 behaviour), followed by severe RSL and CSL with macular sparing only at age 74 (III:3). ON GVF testing, a peripheral island of nasal, inferior and temporal vision was also still present at age 74, but no regional/altitudinal pattern was evident. Also, in $\mathrm{P} 23 \mathrm{H}$ patients, rod-driven ERGs were non-recordable to standard flashes since age 12 (V:2), but could be clearly elicited with brighter stimuli and b-wave splitting into separate cone- and a rod-driven peaks was also seen (loss of rod-driven sensitivity), as previously reported in class-B phenotypes (Iannaccone et al., 2006). This is in line with the partially preserved dark adaptation rod-mediated function on MAP. At this young age, mixed responses were fairly well preserved ( 60\%) and cone ERGs were normal. By age 41, mixed, transient cone, and flicker cone ERGs were reduced to about $10 \%, 45 \%$ and $75 \%$ of the lowest normal limits, respectively. At age 74, although markedly reduced in amplitude and markedly delayed, mixed and cone-driven ERGs (recorded with HK-loop electrodes) remained clearly recordable $(\sim 8 \%, 20 \%$ and $27 \%$ of the lowest normal limits, respectively). Overall, the P23Hassociated phenotype was significantly more severe than the N15S phenotype. 
Visual acuity is well preserved in both mutants, with most patients retaining $\geq 20 / 40$, although substantial inter-individual variability is observed, both between and within mutations. ERG measurements were also performed. Although instances of non-recordable ERGs have been reported in the literature, most patients retain measurable mixed ERG b-waves. Longitudinal measurements in the N15S proband (IV:1) with Class-B1 phenotype show a yearly rate of ERG b-wave loss from baseline of about $6.25 \%$. This is very similar to that reported for of another Class-B1 mutant (P180A, 7\%/yr) (Iannaccone et al., 2006). The ERG is much better preserved in N15S than in $\mathrm{P} 23 \mathrm{H}$.

It has been suggested that cone loss is spatially and temporally related to rod loss and the rate of cone loss is slower than that of rods in Class B mutants (Cideciyan et al., 1998). The in vivo data strongly support this hypothesis. Likewise, coexistence of Class-B1 and B2 features can occur in the same family in association with the $\mathrm{P} 23 \mathrm{H}$ mutation, a transition that appears to be linked to disease stage. Despite evidence of regional disease and retention of rod function, the $\mathrm{P} 23 \mathrm{H}$ mutation causes greater and earlier rod function loss than $\mathrm{N} 15 \mathrm{~S}$, and an overall significantly greater disease severity.

The N15S phenotype is remarkable for the presence of a ring of pericentral disease, severely compromised kinetics of rhodopsin regeneration, and a cone > rod pattern of ERG dysfunction, but later in life rod disease progression continues to precede that of cones as postulated for Class-B phenotypes. 
Figure 7.3 - Representative ISCEV-standard ERG response

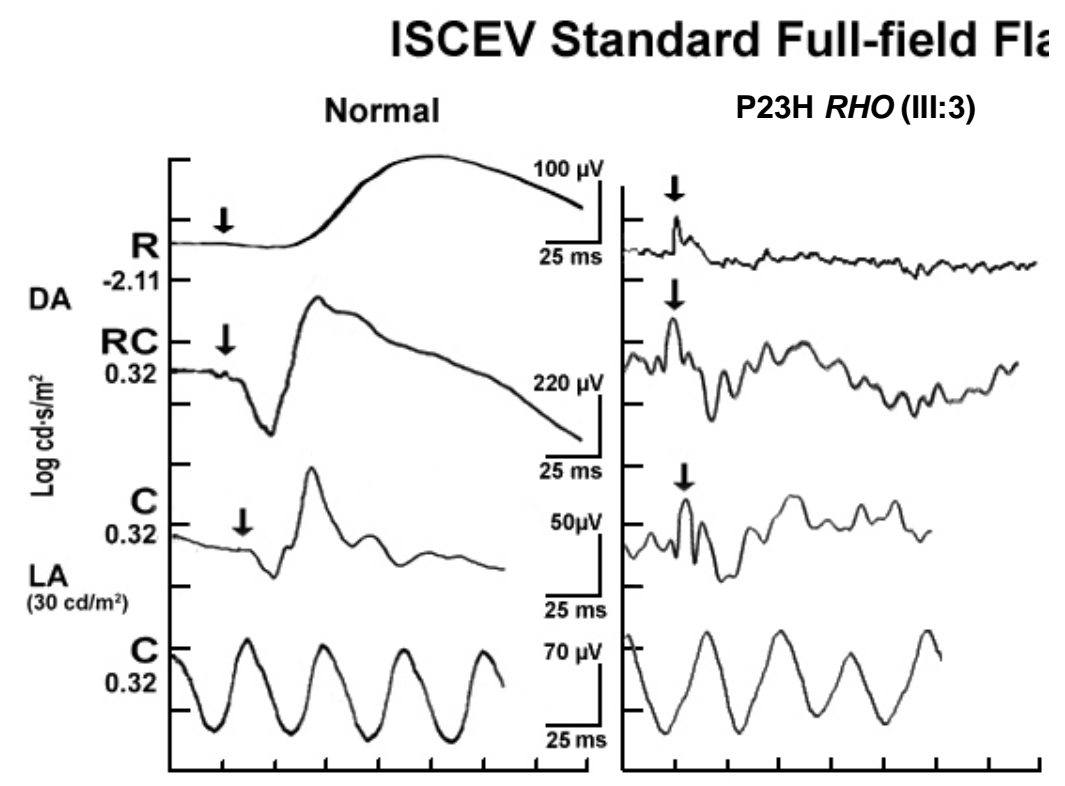

Full-field flash ERG testing of a P23H patient.

SOURCE: Not published. With permission.

\subsubsection{Comparison of $\mathrm{P} 23 \mathrm{H}$ and N15S human patient data with in vitro studies}

The clinical and functional characterizations of the two mutants are compared here to my molecular studies (subcellular localization using confocal microscopy, SDS-PAGE mobility, glycosylation studies, thermal stability and secondary structure content using spectroscopy and Attenuated Total Reflectance Fourier Transform Infrared (ATR/FT-IR), described in detail in Chapter 4). Being able to quantitatively compare the characteristics of the two mutants in vitro and in vivo allowed the study of the extent of severity in molecular and functional terms. The in vivo functional and clinical studies, expression profiles in cell culture and molecular/biophysical in vitro studies of the purified proteins all suggest that the phenotype of $15 \mathrm{~S}$ is significant but is less than that of $\mathrm{P} 23 \mathrm{H}$. The correlation between the relative severities lends support to the hypothesis that the molecular properties of the mutant proteins are directly related to the clinical manifestations in patients. Despite the global classification into the same class, both from a clinical and a molecular perspective, a quantitative comparison reveals that the two mutations differ in severity of both, clinical manifestations, and molecular properties. Although not proving causality, this observation does provide strong support for the hypothesis that the molecular 
properties of the mutant rhodopsin proteins are quantitatively linked to the phenotype observed in patients.

\subsubsection{Comparison of Ce6 effects on RP progression in vivo versus in vitro model systems}

Having established that stability of rhodopsin is related - possibly in a causal manner - to clinical phenotype of RP mutants P23H and N15S (Chapter 3) and also that stability can be enhanced by small molecules (Chapter 4), I then tested if the $\mathrm{P} 23 \mathrm{H}$ phenotype can be rescued by the small molecule stability enhancer $\mathrm{Ce} 6$ in a rat model of $\mathrm{P} 23 \mathrm{H}$ induced $\mathrm{RP}$, described in Chapter 6. The results of ERG and retinal tissue analysis indicated that Ce6 exerts a positive functional effect by slowing the rate of photoreceptor degeneration of $\mathrm{P} 23 \mathrm{H}$ rats in vivo. The studies indicated that Ce6 affects RP progression in vivo and the effects are likely linked to the binding of this molecule to rhodopsin as demonstrated in vitro.

\subsection{FUTURE WORK - PROSPECTS FOR NOVEL APPROACHES TO PREVENT AND TREAT RP IN PATIENTS}

This thesis investigated new approaches to treat RP. The emphasis was to find new treatments of those manifestations of disease caused by mutations in the photoreceptor rhodopsin. To this end the hypothesis was tested that compounds other than retinal can rescue folded rhodopsin and/or reduces photoreceptor cell death by means of exerting a direct effect on the structure and stability of rhodopsin. A conceptual view of the areas where rhodopsin can be targeted is shown schematically in Figure 7.4. 
Figure 7.4 - Schematic representation of the in vitro and in vivo experimental steps taken in this work.

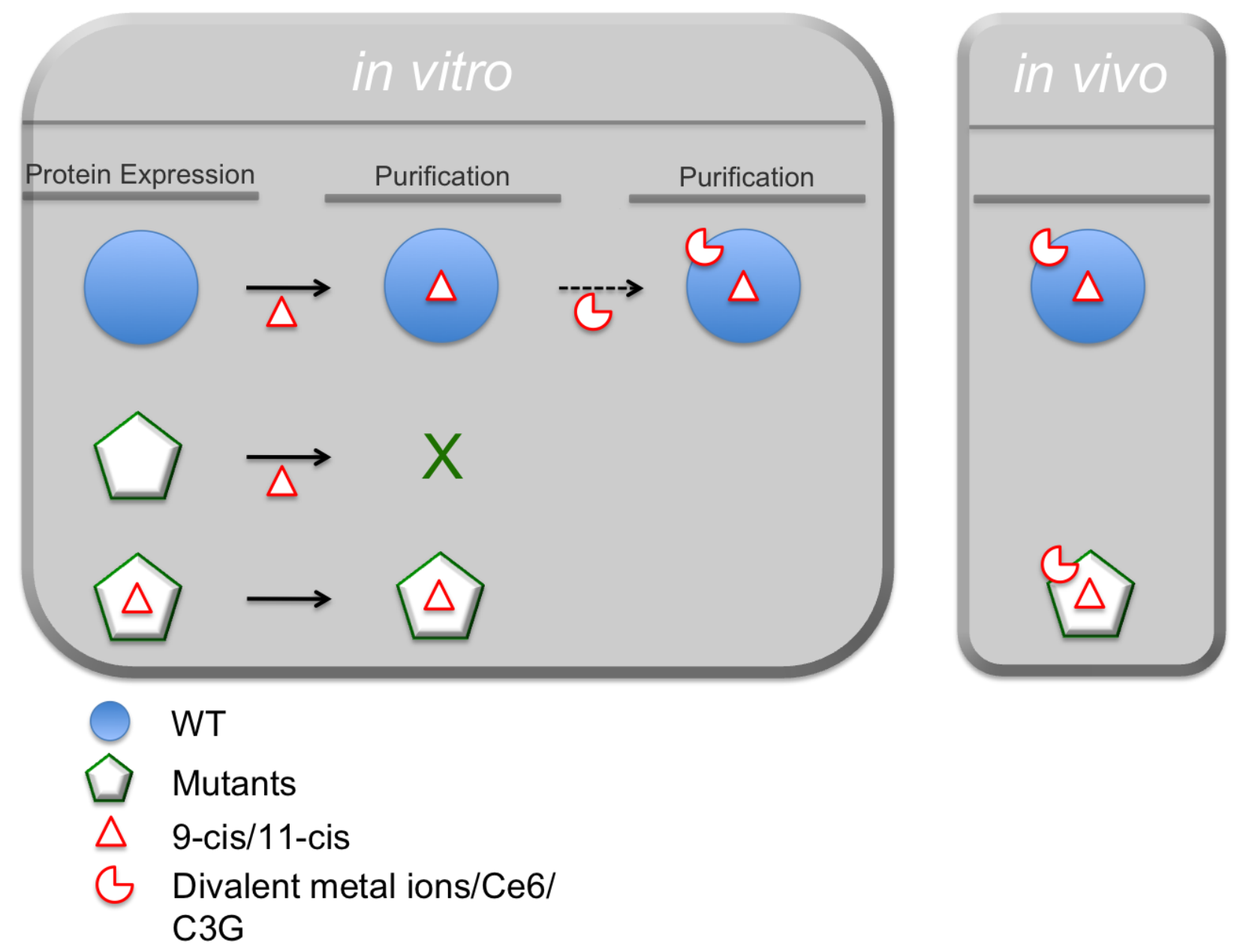

SOURCE: Balen, 2012

It was previously found and confirmed in this thesis for the particular mutants under study that the addition of 9-cis and 11-cis retinal during protein expression in cell culture improves the folding of rhodopsin mutants, increasing the amount of correctly folded rhodopsin. This in vitro result is in agreement with in vivo studies where vitamin A was administered to patients and partially slowed down the RP. However, retinals are able to only partially improve rhodopsin folding and large amounts of vitamin A are toxic. Therefore, in this thesis, other molecules were also tested in vitro and in vivo. Divalent metal ions $\left(\mathrm{Zn}^{2+}, \mathrm{Cu}^{2+}, \mathrm{Fe}^{2+}, \mathrm{Ni}^{2+}, \mathrm{Mg}^{2+}\right.$ and $\left.\mathrm{Mn}^{2+}\right)$, the anthocyanin compound $\mathrm{C} 3 \mathrm{G}$ and the Chlorophyl derivative Ce6 were tested in vitro as a mean of stabilizing the structure of rhodopsin. All these molecules exerted an effect on opsin/rhodopsin structure at different extents and directions (stabilization and destabilization). Metal ions and Ce6 
conferred a pronounced increase in the thermal stability of the secondary structure of rhodopsin. The Ce6 molecule was also tested in light-induced degeneration and hereditary rat models in vivo and has shown an effect on retinal protection. In contrast, $\mathrm{C} 3 \mathrm{G}$ exerted a destabilizing effect on rhodopsin structure while enhancing the regeneration rates and were therefore only tested in vitro. This thesis work has shown that Ce6 but not $\mathrm{C} 3 \mathrm{G}$ can be used to help in the rescue of folded rhodopsin and/or reducing photoreceptor cell death. This provides a novel avenue and a complementary one to current approaches to clinically study the disease and help patients that are affected with hereditary RP.

A number of areas for future exploitation of this finding and further consolidation of its premises are opened up by the work of this thesis. Shown in Figure 7.5 are my suggested areas of concentration for future work.

Figure 7.5 - Suggested concentration areas for future work.

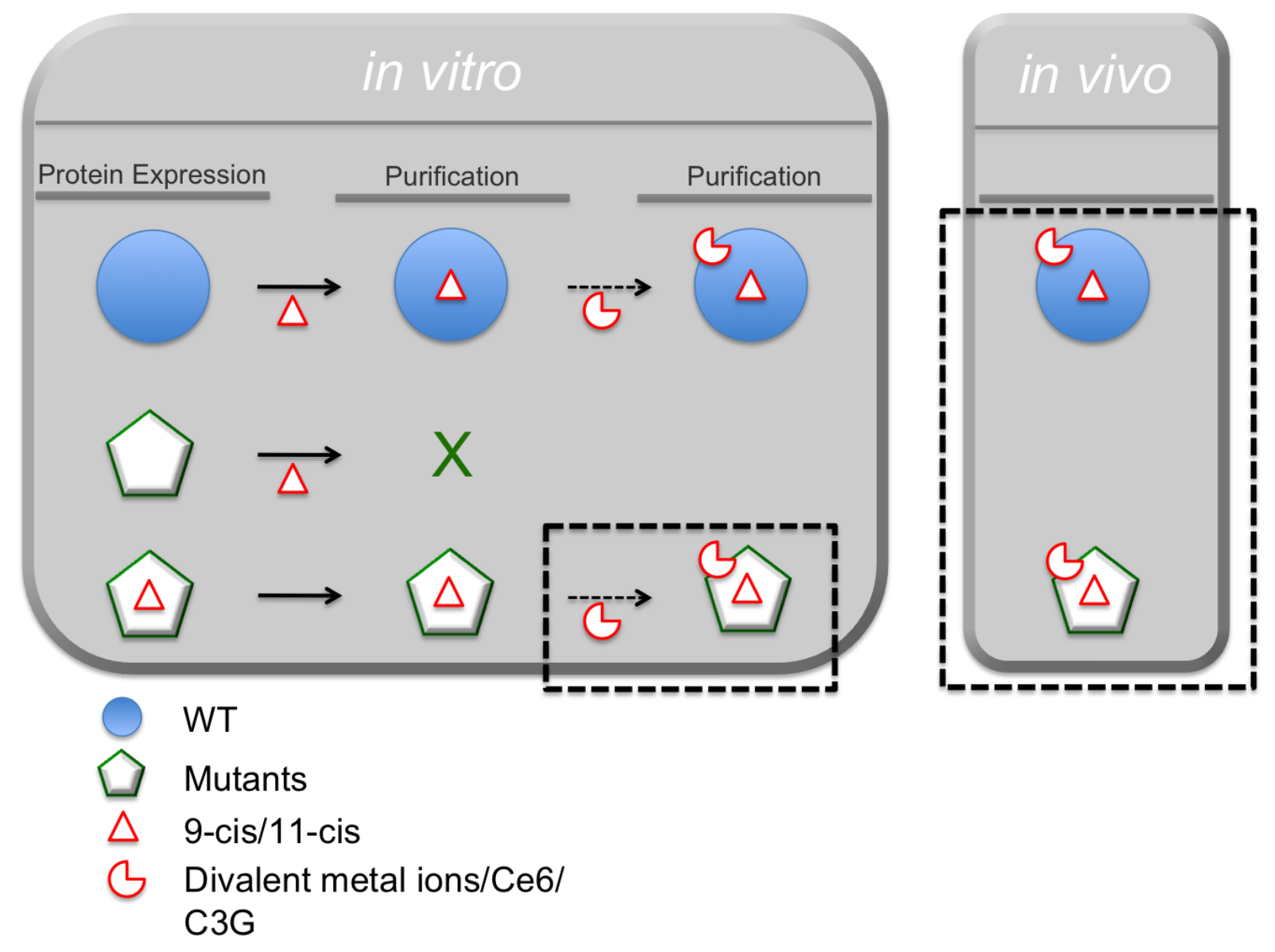

SOURCE : Balen, 2012 
In vivo experiments could be conducted to better evaluate the effect of $\mathrm{Ce} 6$ on the prevention of retinal degeneration caused by light-damage. Factors that could be exploited are the number of rats, which could be increased, dosage and frequency of treatment, as well age at which the treatment is performed that could be varied. The parameters should also be extended to RP affected rats.

In vitro studies need to fill the current gaps opened by the results of my findings. Since addition of 9-cis and 11-cis during the expression of the rhodopsin mutants, $\mathrm{P} 23 \mathrm{H}$ and $\mathrm{N} 15 \mathrm{~S}$, mediated the purification of high amounts of protein, further studies can be pursued where the addition of different molecules, such as the use of divalent metal ions alone and in conjunction with Ce6 can be investigated. To facilitate a direct comparison, the corresponding studies I have done in my thesis with WT rhodopsin need to be carried out with purified mutant proteins. Thus, all in vivo studies have to be matched with the corresponding in vitro studies. Given the promising results obtained in this thesis for WT rhodopsin in vitro for Ce6 alone and the combination of $\mathrm{Ce} 6$ and $\mathrm{Zn} 2+$, and the observation that $\mathrm{Ce} 6$ alone helps the $\mathrm{P} 23 \mathrm{H}$ rats in vivo, strongly suggests, that the proposed approach will be successful.

Finally, to understand the negative results obtained with the in vivo rat model of RP, S334ter upon treatment of Ce6, in vitro analysis could be performed in order to comprehend the mechanism behind the effects of these molecules on the S334ter mutation. Specifically, we propose that Ce6 cannot bind to S334ter. This hypothesis needs to be tested experimentally. The stability of the mutant also needs to be investigated in vitro to see if stabilization in this case by binding of small molecules would be beneficial. Given that Ce6 is not a beneficial molecule for this particular rat model, other compounds, such as $\mathrm{C} 3 \mathrm{G}$ and metal ions could be investigated. 


\subsection{IMPACT}

Analysis of the interaction of various ligands with rhodopsin, results described in Chapter 3 of this thesis, demonstrated that there are different ligands to rhodopsin, other than 11-retinal, that bind to rhodopsin. These are allosteric ligands and are believed to bind at the $\mathrm{CP}$ domain of rhodopsin (Yanamala, 2009). The interaction of these accessory ligands exerted different effects on rhodopsin. $\mathrm{Ce} 6$ and metal ions such as $\mathrm{Zn}^{2+}, \mathrm{Fe}^{2+}, \mathrm{Cu}^{2+}$ have shown to stabilize rhodopsin secondary and tertiary structure. $\mathrm{C} 3 \mathrm{G}$ binding to rhodopsin resulted in the destabilization of retinal-protein interactions, which explains its effect on enhancement of the regeneration rates of rhodopsin. Yet, it was already known that $\mathrm{Zn}^{2+}$ deficiency causes retinal dysfunctions such as night blindness and neurodegeneration. Because $\mathrm{Zn}^{2+}$ binds directly to the photoreceptor rhodopsin and alters its stability, the stabilization of rhodopsin may be key to prevention and treatment of retinal dysfunctions. These findings can be of great potential importance for the treatment of retinal degeneration diseases, where mutations in the photoreceptor rhodopsin are known to be the major disease cause in vivo.

The stabilization obtained by $\mathrm{Ce} 6$ and $\mathrm{Zn}^{2+}$ in vitro and the effect of $\mathrm{Ce} 6$ in vivo can be potentially relevant to dysfunctions caused by nutrient deficiencies, but also for inherited diseases. Retinitis Pigmentosa (RP) is a disease that reflects a large group of genetically heterogeneous disorders marked by early rod photoreceptor dysfunction and progressive degeneration of rods and cones (Lam, 2005). The initial causes for RP are heterogeneous, and mutations in many different genes can lead to RP development (Berson, 1993c; Cooper et al., 1998). The first of these genes that has been identified as a genetic cause for RP was rhodopsin. Now more than 150 mutations in rhodopsin have been identified in different RP patients (Cooper et al., 1998). 42 of these RP mutations have been studied in vitro and 31 of them (>70\%) were found to cause misfolding of rhodopsin (Rader et al., 2004). Since in vitro misfolding and cellbased retention in the endoplasmic reticulum have been proposed to be one of the molecular triggering events associated with retinal degeneration in RP in 1994 (Kaushal and Khorana, 1994) much effort has concentrated on rescuing correctly folded rhodopsin with excess Vitamin A or retinoid derivative. A clinical trial of nutritional supplements for adults with the common forms of RP (Kupfer, 1993) showed that vitamin A treatment alone cannot cure RP (Kupfer, 1993) but maybe additional means of stabilization of rhodopsin could open the way to a new and 
more direct therapeutic tool, applicable to many different mutants of rhodopsin as long as their stabilization contributes to the mechanism of RP prevention/slow-down.

The studies presented here do not only have direct impact on vision. Rhodopsin is a prototypic member of the GPCR family, which accounts for 2 percent of the human genome and is the target for approximately 50 percent of current pharmaceutical compounds (Jacoby et al., 1997). Indeed, $\mathrm{Zn}^{2+}$ has been found to bind also to other members of the GPCR family and has been shown to have physiological roles in some of these receptors. For $\beta 2$-adrenergic, dopamine, and melanocortin receptors, $\mathrm{Zn}^{2+}$ is a positive allosteric modulator of agonist binding (Holst and Schwartz, 2003; Norregaard et al., 1998; Schetz et al., 1999; Schetz and Sibley, 1997). Other studies presented experimental evidence showing that $\mathrm{Zn}^{2+}$ also acts as a signaling molecule (Hershfinkel et al., 2001). Inhibition of G protein activation was observed for rhodopsin (Sheikh et al., 1996), the $\beta 2$-adrenergic receptor and the parathyroid hormone receptor (Sheikh et al., 1999). These are only some GPCRs for which $\mathrm{Zn}^{2+}$ effects have been reported and it is likely that other GPCRs will be affected also. In this thesis, it has been shown that there can be potential benefits in combining $\mathrm{Zn}^{2+}$ with other allosteric modulators, such as $\mathrm{Ce} 6$, to modulate the stability of GPCRs as well as interactions of the GPCRs with their signaling ligands. It is of particular importance to note that $\mathrm{Ce} 6$ is believed to bind in the $\mathrm{CP}$ domain of rhodopsin (Yanamala, 2009), an area that is highly conserved in GPCRs in general. Thus, binding of allosteric CP ligands and stabilization of the respective receptors may be achieved not only in rhodopsin but also other GPCRs. 


\section{REFERENCES*}

Acland GM, Aguirre GD, Ray J, et al. Gene therapy restores vision in a canine model of childhood blindness. Nat Genet. 2001;28(1):92-5.

Aggett PJ, Comerford JG. Zinc and human health. Nutr Rev. 1995 Sep;53(9 Pt 2):S16-22.

Anukanth A, Khorana HG. Structure and function in rhodopsin. Requirements of a specific structure for the intradiscal domain. J Biol Chem. 1994;269(31):19738-44.

Bagchi D, Sen CK, Bagchi M, et al. Anti-angiogenic, antioxidant, and anti-carcinogenic properties of a novel anthocyanin-rich berry extract formula. Biochemistry. 2004;69(1):75-80.

Bainbridge JW, Smith AJ, Barker SS, et al. Effect of gene therapy on visual function in Leber's congenital amaurosis. N Engl J Med. 2008;358(21):2231-9.

Balem F, Yanamala N, Klein-Seetharaman J. Additive effects of chlorin E6 and metal ion binding on the thermal stability of rhodopsin in vitro. Photochem and Photob. 2009:85:471-478

Bennett J, Zeng Y, Bajwa R, et al. Adenovirus-mediated delivery of rhodopsin-promoted bcl-2 results in a delay in photoreceptor cell death in the $\mathrm{rd} / \mathrm{rd}$ mouse. Gene Ther. 1998;5(9):1156-64.

Bennicelli J, Wright JF, Komaromy A, et al. Reversal of blindness in animal models of leber congenital amaurosis using optimized AAV2-mediated gene transfer. Mol Ther. 2008;16(3):45865 .

Berson EL. Retinitis Pigmentosa. The Friedenwald Lecture. Invest Opthalmol Vis Sci. 1993c;34:1659-76.

Berson EL, Rosner B, Sandberg MA, et al. A randomized trial of vitamin A and vitamin E supplementation for retinitis pigmentosa. Arch Ophthalmol. 1993a;111(6):761-72.

Berson EL, Rosner B, Sandberg MA, et al. Vitamin A supplementation for retinitis pigmentosa. Arch Ophthalmol. 1993b;111(11):1456-9.

Berson EL, Rosner B, Sandberg MA, et al. Clinical trial of lutein in patients with retinit is pigmentosa receiving vitamin A. Arch Ophthalmol. 2010;128(4):403-11.

Berson EL, Rosner B, Sandberg MA, et al. Further evaluation of docosahexaenoic acid in patients with retinitis pigmentosa receiving vitamin A treatment: subgroup analyses. Arch Ophthalmol. 2004;122(9):1306-14.

*In according with: International Committee of Medical Journal Editors. The recommended style for references is based on the National Information Standards Organization NISO Z39.29-2005 (R2010). Available from: http://www.nlm.nih.gov/bsd/uniform_requirements.html [2011 July 15]. 
Bok D, Yasumura D, Matthes MT, et al. Effects of adeno-associated virus-vectored ciliary neurotrophic factor on retinal structure and function in mice with a P216L rds/peripherin mutation. Exp Eye Res. 2002;74(6):719-35.

Brouillard R, Dubois JE. Mechanism of Structural Transformations of Anthocyanins in Acidic Media. J Am Chem Soc. 1977;99(5):1359-64.

Bullock AN, Debreczeni JE, Fedorov OY, et al. Structural basis of inhibitor specificity of the human protooncogene proviral insertion site in moloney murine leukemia virus (PIM-1) kinase. J Med Chem. 2005;48(24):7604-14.

Cajal SR. Histologie Du Système Nerveux de l'Homme et Des Vertébrés. 1911. Available from http://en.wikipedia.org/wiki/Retina. Taken on December 07, 2011.

Caspi A, Dorn JD, McClure KH, et al. Feasibility study of a retinal prosthesis: spatial vision with a 16-electrode implant. Arch Ophthalmol. 2009;127(4):398-401.

Chabre M, Bigay J, Bruckert F, et al. Visual signal transduction: the cycle of transducin shuttling between rhodopsin and cGMP phosphodiesterase. Cold Spring Harb Symp Quant Biol. 1988;53 Pt 1:313-24.

Chang G, Hao Y, Wong F. Apoptosis: final common pathway of photoreceptor death in rd, rds, and rhodopsin mutant mice. Neuron. 1993;11:595-605.

Chen C, Okayama H. High-efficiency transformation of mammalian cells by plasmid DNA. Mol Cell Biol. 1987;7(8):2745-52.

Chow AY, Chow VY. Subretinal electrical stimulation of the rabbit retina. Neurosci Lett. 1997;225(1):13-6.

Cideciyan AV, Hood DC, Huang Y, et al. Disease sequence from mutant rhodopsin allele to rod and cone photoreceptor degeneration in man. Proc Natl Acad Sci U S A. 1998;95(12):7103-8.

Cooper DN, Ball EV, Stenson PD, et al. The Human gene mutation database at the institute of medical genetics in Cardiff. BIOBASE - Biological Database; 2007 [updated May 3, 2012]; Available from: www.hgmd.org.

Cruickshanks KJ, Klein R, Klein BE. Sunlight and age-related macular degeneration. The Beaver Dam Eye Study. Arch Ophthalmol. 1993 Apr;111(4):514-8.

Darwin C. On the Origin of Species by Means of Natural Selection. Editions DT, editor. Mineola, New York: Dover Publications, INC; 2006.

Dong CJ, Hare WA. GABAc feedback pathway modulates the amplitude and kinetics of ERG bwave in a mammalian retina in vivo. Vision Res. 2002;42(9):1081-7.

Douglas RH, Partridge JC, Dulai K, et al. Dragon fish see using chlorophyll. Nature. 1998;393:423-4. 
Douglas RH, Partridge JC, Dulai KS, et al. Enhanced retinal longwave sensitivity using a chlorophyll-derived photosensitiser in Malacosteus niger, a deep-sea dragon fish with far red bioluminescence. Vision Research. 1999;39(17):2817-32.

Drenser KA, Timmers AM, Hauswirth WW, et al. Ribozyme-targeted destruction of RNA associated with autosomal-dominant retinitis pigmentosa. Invest Ophthalmol Vis Sci. 1998 Apr;39(5):681-9.

Dryja TP, Berson EL, Rao VR, et al. Heterozygous missense mutation in the rhodopsin gene as a cause of congenital stationary night blindness. Nat Genet. [10.1038/ng0793-280]. 1993;4(3):2803.

Dryja TP, Finn JT, Peng YW, et al. Mutations in the gene encoding the alpha subunit of the rod cGMP-gated channel in autosomal recessive retinitis pigmentosa. Proc Natl Acad Sci U S A. 1995;92(22):10177-81.

Dryja TP, McGee TL, Reichel E, et al. A point mutation of the rhodopsin gene in one form of retinitis pigmentosa. Nature. 1990;343(6256):364-6.

Faktorovich E, Steinberg R, Yasumura D, et al. Photoreceptor degeneration in inherited retinal dystrophy delayed by basic fibroblast growth factor. Nature. 1990;347:83 - 6 .

Farrens DL, Khorana HG. Structure and Function in Rhodopsin : Measurement of the rate of metarhodopsin II decay by Fluorescence Spectroscopy. JBC. 1995;270(10):5073-6.

Fernandez-Sanchez L, Lax P, Pinilla I, et al. Tauroursodeoxycholic acid prevents retinal degeneration in transgenic P23H rats. Invest Ophthalmol Vis Sci. 2011;52(8):4998-5008.

Fleisch VC, Jametti T, Neuhauss SCF. Electroretinogram (ERG) Measurements in Larval Zebrafish. CSH Protocols. 2008.

Foster RG, Provencio I, Hudson D, et al. Circadian photoreception in the retinally degenerate mouse (rd/rd). J Comp Physiol A. 1991;169(1):39-50.

Franze K, Grosche J, Skatchkov SN, et al. Muller cells are living optical fibers in the vertebrate retina. Proc Natl Acad Sci U S A. 2007;104(20):8287-92.

Frasson M, Picaud S, Leveillard T, et al. Glial cell line-derived neurotrophic factor induces histologic and functional protection of rod photoreceptors in the $\mathrm{rd} / \mathrm{rd}$ mouse. Invest Ophthalmol Vis Sci. 1999;40(11):2724-34.

Frishman D, Argos P. Knowledge-based protein secondary structure assignment. Proteins. 1995 Dec;23(4):566-79.

Gal A, Li Y, Thompson DA, et al. Mutations in MERTK, the human orthologue of the RCS rat retinal dystrophy gene, cause retinitis pigmentosa. Nat Genet. 2000;26(3):270-1. 
Garriga P, Liu X, Khorana HG. Structure and function in rhodopsin: correct folding and misfolding in point mutants at and in proximity to the site of the retinitis pigmentosa mutation Leu-125--> Arg in the transmembrane helix C. Proc Natl Acad Sci U S A. 1996;93(10):4560-4.

Gearhart PM, Gearhart C, Thompson DA, et al. Improvement of visual performance with intravitreal administration of 9-cis-retinal in Rpe65-mutant dogs. Arch Ophthalmol. 2010;128(11):1442-8.

Glybina IV, Kennedy A, Ashton P, et al. Intravitreous delivery of the corticosteroid fluocinolone acetonide attenuates retinal degeneration in S334ter-4 rats. Invest Ophthalmol Vis Sci. 2010 ;51(8):4243-52.

Grahn BH, Paterson PG, Gottschall-Pass KT, et al. Zinc and the eye. J Am Coll Nutr. 2001;20(2 Suppl):106-18.

Hamel C. Retinitis pigmentosa. Orphanet J Rare Dis. 2006;1:40.

Harazny J, Scholz M, Buder T, et al. Electrophysiological deficits in the retina of the DBA/2J mouse. Doc Ophthalmol. 2009;119(3):181-97.

Hargrave PA, McDowell JH, Curtis DR, et al. The structure of bovine rhodopsin. Biophys Struct Mech. 1983;9(4):235-44.

Hargrave PA. The amino-terminal tryptic peptide of bovine rhodopsin. A glycopeptide containing two sites of oligosaccharide attachment. Biochim Biophys Acta. 1977;492(1):83-94.

Hauswirth WW, Aleman TS, Kaushal S, et al. Treatment of leber congenital amaurosis due to RPE65 mutations by ocular subretinal injection of adeno-associated virus gene vector: short-term results of a phase I trial. Hum Gene Ther. 2008;19(10):979-90.

Hershfinkel M, Moran A, Grossman N, et al. A zinc-sensing receptor triggers the release of intracellular Ca2+ and regulates ion transport. Proc Natl Acad Sci U S A. 2001;98(20):11749-54.

Holst B, Schwartz TW. Molecular mechanism of agonism and inverse agonism in the melanocortin receptors: $\mathrm{Zn}(2+)$ as a structural and functional probe. Ann N Y Acad Sci. 2003;994:1-11.

Humayun MS, de Juan E, Jr. Artificial vision. Eye (Lond). 1998;12 ( Pt 3b):605-7.

Humphrey W, Dalke A, Schulten K. VMD: visual molecular dynamics. J Mol Graph. 1996;14(1):33-8, 27-8.

Iannaccone A, Man D, Waseem N, et al. Retinitis pigmentosa associated with rhodopsin mutations: Correlation between phenotypic variability and molecular effects. Vision Res. 2006;46(27):4556-67.

Isayama T, Alexeev D, Makino CL, et al. An accessory chromophore in red vision. Nature. 2006;443(7112):649. 
Jacoby E, Boudon A, Kucharczyk N, et al. A structural rationale for the design of water soluble peptide-derived neurokinin-1 antagonists. J Recept Signal Transduct Res. 1997;17(6):855-73.

Kannabiran C. Retinitis pigmentosa: genetics and gene-based approaches to therapy. Expert Review of Ophthalmology. [Review]. 2008;3(4):417-29.

Karcioglu ZA, Stout R, Hahn HJ. Serum zinc levels in retinitis pigmentosa. Curr Eye Res. 1984;3(8):1043-8.

Kaushal S, Khorana HG. Structure and function in rhodopsin. 7. Point mutations associated with autosomal dominant retinitis pigmentosa. Biochemistry. 1994a;33(20):6121-8.

Kaushal S, Ridge KD, Khorana HG. Structure and function in rhodopsin: the role of asparaginelinked glycosylation. Proc Natl Acad Sci U S A. 1994b;91(9):4024-8.

Khorana HG. Molecular biology of light transducin by the mammalian photoreceptor rhodopsin. J Biomol Structure and Dynamics. 2000;11:1-16.

Kohn J, Wilchek M. A colorimetric method for monitoring activation of Sepharose by cyanogen bromide. Biochemical and biophysical research communications. 1978;84(1):7-14.

Kolb H. WEBVISION - The organization of the retina and visual system: University of Utah; 2011. Available from: http://webvision.med.utah.edu/book/.

Kraft SP, Parker JA, Matuk Y, et al. The rat electroretinogram in combined zinc and vitamin A deficiency. Invest Ophthalmol Vis Sci. 1987;28(6):975-84.

Kranich H, Bartkowski S, Denton MJ, et al. Autosomal dominant 'sector' retinitis pigmentosa due to a point mutation predicting an Asn-15-Ser substitution of rhodopsin. Hum Mol Genet. 1993;2(6):813-4.

Krebs MP, Holden DC, Joshi P, et al. Molecular mechanisms of rhodopsin retinitis pigmentosa and the efficacy of pharmacological rescue. J Mol Biol. 2010;395(5):1063-78.

Kumel G, Daus H, Mauch H. Improved method for the cyanogen bromide activation of agarose beads. Journal of Chromatography. 1979;172:221-6.

Kupfer C. Information for Doctors Who Follow Patients with Retinitis Pigmentosa. National Eye Institute; 1993; Available from: http://www.nei.nih.gov/news/clinicalalerts/alert-rp.asp.

Lam BL. Electrophysiology of vision: Clinical testing and application. Boca Raton: Taylor and Francis; 2005.

LaVail MM, Battelle BA. Influence of eye pigmentation and light deprivation on inherited retinal dystrophy in the rat. Exp Eye Res. 1975;21:167-92.

Lengyel I, Peto T. Cure or cause: opposing roles for zinc in age-related macular degeneration. Expert Rev Ophthalmol. 2008;3(1):1-4. 
Leonard KC, Petrin D, Coupland SG, et al. XIAP protection of photoreceptors in animal models of retinitis pigmentosa. PLoS One. 2007;2(3):e314.

Lewin AS, Drenser KA, Hauswirth WW, et al. Ribozyme rescue of photoreceptor cells in a transgenic rat model of autosomal dominant retinitis pigmentosa. Nat Med. 1998;4(8):967-71.

Li Y, Tao W, Luo L, et al. CNTF induces regeneration of cone outer segments in a rat model of retinal degeneration. PLoS One. 2010;5(3):e9495.

Liang FQ, Aleman TS, Dejneka NS, et al. Long-term protection of retinal structure but not function using RAAV.CNTF in animal models of retinitis pigmentosa. Mol Ther. 2001;4(5):461 72 .

Lila MA. Anthocyanins and Human Health: An In Vitro Investigative Approach. J Biomed Biotechnol. 2004;(5):306-13.

Liu C, Li Y, Peng M, et al. Activation of caspase-3 in the retina of transgenic rats with the rhodopsin mutation s334ter during photoreceptor degeneration. J Neurosci. 1999;19(12):4778-85.

Liu X, Garriga P, Khorana HG. Structure and function in rhodopsin: correct folding and misfolding in two point mutants in the intradiscal domain of rhodopsin identified in retinitis pigmentosa. Proc Natl Acad Sci U S A. 1996;93(10):4554-9.

Lucas RJ, Douglas RH, Foster RG. Characterization of an ocular photopigment capable of driving pupillary constriction in mice. Nat Neurosci. 2001;4(6):621-6.

Lund RD, Wang S, Klimanskaya I, et al. Human embryonic stem cell-derived cells rescue visual function in dystrophic RCS rats. Cloning Stem Cells. 2006 Fall;8(3):189-99.

MacLaren RE, Pearson RA, MacNeil A, et al. Retinal repair by transplantation of photoreceptor precursors. Nature. 2006;444(7116):203-7.

Maeda A, Maeda T, Palczewski K. Improvement in rod and cone function in mouse model of Fundus albipunctatus after pharmacologic treatment with 9-cis-retinal. Invest Ophthalmol Vis Sci. 2006;47(10):4540-6.

Maguire AM, Simonelli F, Pierce EA, et al. Safety and efficacy of gene transfer for Leber's congenital amaurosis. N Engl J Med. 2008 May 22;358(21):2240-8.

Maguire AM, High KA, Auricchio A, et al. Age-dependent effects of RPE65 gene therapy for Leber's congenital amaurosis: a phase 1 dose-escalation trial. Lancet. 2009;374(9701):1597-605.

Malien-Aubert C, Dangles O, Amiot MJ. Color stability of commercial anthocyanin-based extracts in relation to the phenolic composition. Protective effects by intra- and intermolecular copigmentation. J Agric Food Chem. 2001;49(1):170-6.

Marmor M, Arden G, Nilsson S, et al. Standard for clinical electroretinography. Arch Ophthalmol. 1989;107:816-9. 
Martinez-Navarrete G, Seiler MJ, Aramant RB, et al. Retinal degeneration in two lines of transgenic S334ter rats. Exp Eye Res. 2011;92(3):227-37.

Matsumoto H, Inaba H, Kishi M, et al. Orally administered delphinidin 3-rutinoside and cyanidin 3-rutinoside are directly absorbed in rats and humans and appear in the blood as the intact forms. J Agric Food Chem. 2001;49(3):1546-51.

Matsumoto H, Nakamura Y, Iida H, et al. Comparative assessment of distribution of blackcurrant anthocyanins in rabbit and rat ocular tissues. Exp Eye Res. 2006;83(2):348-56.

Matsumoto H, Nakamura Y, Tachibanaki S, et al. Stimulatory effect of cyanidin 3-glycosides on the regeneration of rhodopsin. J Agric Food Chem. 2003;51(12):3560-3.

Molavi DW. Neuroscience Tutorial. Washington University Program in Neuroscience 1997; Available from: http://thalamus.wustl.edu/course/eyeret.html.

Musarella MA, Macdonald IM. Current concepts in the treatment of retinitis pigmentosa. J Ophthalmol. 2011;2011:753547.

Muth ER, Laurent JM, Jasper P. The effect of bilberry nutritional supplementation on night visual acuity and contrast sensitivity. Altern Med Rev. 2000;5(2):164-73.

Nakaishi H, Matsumoto H, Tominaga S, et al. Effects of black current anthocyanoside intake on dark adaptation and VDT work-induced transient refractive alteration in healthy humans. Altern Med Rev. 2000 Dec;5(6):553-62.

Noel WK, Walker VS, Kang BSK, et al. Retinal damage by light in rats. Investigative Ophthalmology and Visual Science 1966 5: 450-473

Noel WK and Albrecht R. Irreversible effects of visible light on the retina: Role of vitamin A. Science 1971: 172-176

Norregaard L, Frederiksen D, Nielsen EO, et al. Delineation of an endogenous zinc-binding site in the human dopamine transporter. Embo J. 1998;17(15):4266-73.

Okada T, Fujiyoshi Y, Silow M, et al. Functional role of internal water molecules in rhodopsin revealed by X-ray crystallography. Proc Natl Acad Sci U S A. 2002;99(9):5982-7.

Ollesch J, Kunnemann E, Glockshuber R, et al. Prion protein alpha-to-beta transition monitored by time-resolved Fourier transform infrared spectroscopy. Appl Spectrosc. 2007;61(10):1025-31.

O'Mahoney JV, Adams TE. Optimization of experimental variables influencing reporter gene expression in hepatoma cells following calcium phosphate transfection. DNA Cell Biol. 1994;13(12):1227-32.

Oprian DD, Molday RS, Kaufman RJ, et al. Expression of a synthetic bovine rhodopsin gene in monkey kidney cells. Proc Natl Acad Sci U S A. 1987;84(24):8874-8. 
Organisciak DT, Darrow RM, Barsalou L, et al. Susceptibility to retinal light damage in transgenic rats with rhodopsin mutations. Invest Ophthalmol Vis Sci. 2003;44(2):486-92.

Organisciak DT, Darrow RM, Jiang YI, et al. Protection by dimethylthiourea against retinal light damage in rats. Invest Ophthalmol Vis Sci. 1992;33(5):1599-609.

Organisciak DT, Wang HM, Li ZY, et al. The protective effect of ascorbate in retinal light damage of rats. Invest Ophthalmol Vis Sci. 1985;26(11):1580-8.

Ovchinnikov Yu A, Abdulaev N, G, Feigina M, Y, et al. The complete Amino Acid Sequence of Visual Rhodopsin. Bioorg Khim. 1982;8:1011-4.

Palczewski K, Kumasaka T, Hori T, et al. Crystal structure of rhodopsin: A G protein-coupled receptor. Science. 2000 Aug 4;289(5480):739-45.

Park PS, Sapra KT, Kolinski M, et al. Stabilizing effect of Zn2+ in native bovine rhodopsin. J Biol Chem. 2007;282(15):11377-85.

Patel AB, Crocker E, Reeves PJ, et al. Changes in Interhelical Hydrogen Bonding upon Rhodopsin Activation J Mol Biol. 2005;347(4):803-12.

Perlman I. The Electroretinogram: ERG. 2011 [June 15 2011]; Available from: http://retina.umh.es/webvision/ERG.html.

Petrin D, Baker A, Coupland SG, et al. Structural and functional protection of photoreceptors from MNU-induced retinal degeneration by the X-linked inhibitor of apoptosis. Invest Ophthalmol Vis Sci. 2003;44(6):2757-63.

Pinilla I, Lund RD, Sauve Y. Enhanced cone dysfunction in rats homozygous for the P23H rhodopsin mutation. Neurosci Lett. 2005;382(1-2):16-21.

Portera-Cailliau C, Sung C, Nathans J, et al. Apoptotic photoreceptor cell death in mouse models or retinitis pigmentosa. Proc Natl Acad Sci. 1994;91:974-8.

Provencio I, Rodriguez IR, Jiang G, et al. A novel human opsin in the inner retina. J Neurosci. 2000;20(2):600-5.

Rader AJ, Anderson G, Isin B, et al. Identification of core amino acids stabilizing rhodopsin. Proc Natl Acad Sci U S A. 2004;101(19):7246-51.

Radtke ND, Aramant RB, Petry HM, et al. Vision improvement in retinal degeneration patients by implantation of retina together with retinal pigment epithelium. Am J Ophthalmol. 2008;146(2):172-82.

Ranchon I, Chen S, Alvarez K, et al. Systemic administration of phenyl-N-tert-butylnitrone protects the retina from light damage. Invest Ophthalmol Vis Sci. 2001;42(6):1375-9. 
Ranchon I, LaVail MM, Kotake Y, et al. Free radical trap phenyl-N-tert-butylnitrone protects against light damage but does not rescue $\mathrm{P} 23 \mathrm{H}$ and $\mathrm{S} 334$ ter rhodopsin transgenic rats from inherited retinal degeneration. J Neurosci. 2003;23(14):6050-7.

Reeves PJ, Callewaert N, Contreras R, et al. Structure and function in rhodopsin: high-level expression of rhodopsin with restricted and homogeneous $\mathrm{N}$-glycosylation by a tetracyclineinducible N-acetylglucosaminyltransferase I-negative HEK293S stable mammalian cell line. Proc Natl Acad Sci U S A. 2002a;99(21):13419-24.

Reeves PJ, Thurmond RL, Khorana HG. Structure and function in rhodopsin: high level expression of a synthetic bovine opsin gene and its mutants in stable mammalian cell lines. Proc Natl Acad Sci U S A. 1996;93(21):11487-92.

Reeves PJ, Kim JM, Khorana HG. Structure and function in rhodopsin: a tetracycline-inducible system in stable mammalian cell lines for high-level expression of opsin mutants. Proc Natl Acad Sci U S A. 2002b;99(21):13413-8.

Reme C, Grimm C, Hafezy F, et al. Apoptotic cell death in retinal degenerations. Prog Retin Eye Res. 1998;17:443-64.

Ridge KD, Lu Z, Liu X, et al. Structure and function in rhodopsin. Separation and characterization of the correctly folded and misfolded opsins produced on expression of an opsin mutant gene containing only the native intradiscal cysteine codons. Biochemistry. $1995 ; 34(10): 3261-7$.

Rivas MA, Vecino E. Animals models and different therapies for treatment of retinitis pigmentosa. Histology and Histopathology. 2009;24:1295-322.

Saliba RS, Munro PM, Luthert PJ, et al. The cellular fate of mutant rhodopsin: quality control, degradation and aggresome formation. J Cell Sci. 2002 :115(Pt 14):2907-18.

Sapra KT, Park PS, Filipek S, et al. Detecting molecular interactions that stabilize native bovine rhodopsin. Journal of Molec Biol. 2006;358:255-69

Sapra KT, Park PS, Palczewski K, et al. Mechanical properties of bovine rhodopsin and bacteriorhodopsin: possible roles in folding and function. Langmuir. 2008;24(4):1330-7.

Schetz JA, Chu A, Sibley DR. Zinc modulates antagonist interactions with D2-like dopamine receptors through distinct molecular mechanisms. J Pharmacol Exp Ther. 1999;289(2):956-64.

Schetz JA, Sibley DR. Zinc allosterically modulates antagonist binding to cloned D1 and D2 dopamine receptors. J Neurochem. 1997;68(5):1990-7.

Sekirnjak C, Jepson LH, Hottowy P, et al. Changes in physiological properties of rat ganglion cells during retinal degeneration. J Neurophysiol. 2011;105(5):2560-71. 
Sheikh SP, Vilardarga JP, Baranski TJ, et al. Similar structures and shared switch mechanisms of the beta2-adrenoceptor and the parathyroid hormone receptor. $\mathrm{Zn}$ (II) bridges between helices III and VI block activation. J Biol Chem. 1999;274(24):17033-41.

Sheikh SP, Zvyaga TA, Lichtarge O, et al. Rhodopsin activation blocked by metal-ion-binding sites linking transmembrane helices C and F. Nature. 1996;383(6598):347-50.

Shuster TA, Martin F, Nagy AK. Zinc causes an apparent increase in rhodopsin phosphorylation. Curr Eye Res. 1996;15(10):1019-24.

Shuster TA, Nagy AK, Conly DC, et al. Direct zinc binding to purified rhodopsin and disc membranes. Biochem J. 1992;282 ( Pt 1):123-8.

Sieving PA, Caruso RC, Tao W, et al. Ciliary neurotrophic factor (CNTF) for human retinal degeneration: phase I trial of CNTF delivered by encapsulated cell intraocular implants. Proc Natl Acad Sci U S A. 2006;103(10):3896-901.

Sieving PA. Retinitis Pigmentosa and Related Disease. Free Medical Text Book 2010.

Sreerama N, Woody RW. Estimation of protein secondary structure from circular dichroism spectra: comparison of CONTIN, SELCON, and CDSSTR methods with an expanded reference set. Anal Biochem. 2000;287(2):252-60.

Steinberg RH, Flannery JG, Naash M, et al. Transgenic rat models of inherited retinal degeneration caused by mutant opsin genes. Invest Ophthalmol Vis Sci 1996;37.

Stojanovic A, Hwa J. Rhodopsin and retinitis pigmentosa: shedding light on structure and function. Receptors Channels. 2002;8(1):33-50.

Stojanovic A, Stitham J, Hwa J. Critical role of transmembrane segment zinc binding in the structure and function of rhodopsin. J Biol Chem. 2004;279(34):35932-41.

Sung CH, Davenport CM, Hennessey JC, et al. Rhodopsin mutations in autosomal dominant retinitis pigmentosa. Proc Natl Acad Sci U S A. 1991;88(15):6481-5.

Sung CH, Davenport CM, Nathans J. Rhodopsin mutations responsible for autosomal dominant retinitis pigmentosa. Clustering of functional classes along the polypeptide chain. J Biol Chem. 1993;268(35):26645-9.

Tam BM, Moritz OL. The role of rhodopsin glycosylation in protein folding, trafficking, and light-sensitive retinal degeneration. J Neurosci. 2009;29(48):15145-54.

Tan MH, Smith AJ, Pawlyk B, et al. Gene therapy for retinitis pigmentosa and Leber congenital amaurosis caused by defects in AIPL1: effective rescue of mouse models of partial and complete Aipl1 deficiency using AAV2/2 and AAV2/8 vectors. Hum Mol Genet. 2009;18(12):2099-114. 
Tanito M, Kaidzu S, Anderson RE. Delayed loss of cone and remaining rod photoreceptor cells due to impairment of choroidal circulation after acute light exposure in rats. Invest Ophthalmol Vis Sci. 2007a;48(4):1864-72.

Tanito M, Li F, Elliott MH, et al. Protective effect of TEMPOL derivatives against light-induced retinal damage in rats. Invest Ophthalmol Vis Sci. 2007b;48(4):1900-5.

Tanito M, Masutani H, Nakamura H, et al. Attenuation of retinal photooxidative damage in thioredoxin transgenic mice. Neurosci Lett. 2002a Jun 28;326(2):142-6.

Tanito M, Masutani H, Nakamura H, et al. Cytoprotective effect of thioredoxin against retinal photic injury in mice. Invest Ophthalmol Vis Sci. 2002b;43(4):1162-7.

Tao W, Wen R, Goddard MB, et al. Encapsulated cell-based delivery of CNTF reduces photoreceptor degeneration in animal models of retinitis pigmentosa. Invest Ophthalmol Vis Sci. 2002;43(10):3292-8.

Tastan $\mathrm{O}, \mathrm{Yu}$ E, Ganapathiraju M, et al. Comparison of stability predictions and simulated unfolding of rhodopsin structures. Photochem Photobiol. 2007;83(2):351-62.

Tirupula KC, Balem F, Yanamala N, et al. pH-dependent interaction of rhodopsin with cyanidin3-glucoside. 2. Functional aspects. Photochem Photobiol. 2009;85(2):463-70.

Tirupula KC. Structure-function studies of the metabotropic glutamate receptor type 6 (mglur6) and comparison with rhodopsin. Pittsburgh: University of Pittsburgh; 2011.

Toledo D, Cordomi A, Proietti MG, et al. Structural Characterization of a zinc high-affinity binding site in rhodopsin. 13th International Conference on Retinal Proteins; June 15-19; Barcelona, Spain2008.

Tomita H, Kotake Y, Anderson RE. Mechanism of protection from light-induced retinal degeneration by the synthetic antioxidant phenyl-N-tert-butylnitrone. Invest Ophthalmol Vis Sci. 2005;46(2):427-34.

Ugarte M, Osborne NN. Zinc in the retina. Progress in Neurobiology. 2001;64(3):219-49.

Valle LJd, Ramon E, Cañavate X, et al. Zinc-induced Decrease of the Thermal Stability and Regeneration of Rhodopsin. J Biol Chem. 2003;278(7):4719-24.

Vedadi M, Niesen FH, Allali-Hassani A, et al. Chemical screening methods to identify ligands that promote protein stability, protein crystallization, and structure determination. Proc Natl Acad Sci U S A. 2006;103(43):15835-40.

Veitch NC, Grayer RJ. Flavonoids and their glycosides, including anthocyanins. Nat Prod Rep. 2008;25(3):555-611.

Veske A, Nilsson SE, Narfstrom K, et al. Retinal dystrophy of Swedish briard/briard-beagle dogs is due to a 4-bp deletion in RPE65. Genomics. 1999;57(1):57-61. 
Wald G, Brown PK. The molar extinction of rhodopsin. J Gen Physiol. 1953;37(2):189-200.

Wald G. Molecular basis of visual excitation. Science. 1968;162(850):230-9.

Wang M, Lam TT, Tso MO, et al. Expression of a mutant opsin gene increases the susceptibility of the retina to light damage. Vis Neurosci. 1997;14(1):55-62.

Washington I, Brooks C, Turro NJ, et al. Phorphyrins as photosensitizers to enhance night vision. J Am Chem Soc. 2004;126(32):9892-3.

Washington I, Zhou J, Jockusch S, et al. Chlorophyll derivatives as visual pigments for super vision in the red. Photochem Photobiol Sci. 2007;6(7):775-9.

Weber M, Rabinowitz J, Provost N, et al. Recombinant adeno-associated virus serotype 4 mediates unique and exclusive long-term transduction of retinal pigmented epithelium in rat, dog, and nonhuman primate after subretinal delivery. Mol Ther. 2003;7(6):774-81.

Wenzel A, Grimm C, Samardzija M, et al. Molecular mechanisms of light-induced photoreceptor apoptosis and neuroprotection for retinal degeneration. Prog Ren Eye Res. 2005 24(2), 275-306.

Yanai D, Weiland JD, Mahadevappa M, et al. Visual performance using a retinal prosthesis in three subjects with retinitis pigmentosa. Am J Ophthalmol. 2007;143(5):820-7.

Yanamala N, Tirupula KC, Balem F, et al. pH-dependent interaction of rhodopsin with cyanidin3-glucoside. 1. Structural aspects. Photochem Photobiol. 2009;85(2):454-62.

Yanamala N. Allosteric Modulation of G Protein Coupled Receptors [Thesis]. Pittsburgh: University of Pittsburgh; 2009.

Yao F, Svensjo T, Winkler T, et al. Tetracycline repressor, tetR, rather than the tetR-mammalian cell transcription factor fusion derivatives, regulates inducible gene expression in mammalian cells. Hum Gene Ther. 1998;9(13):1939-50.

Zafra-Stone S, Yasmin T, Bagchi M, et al. Berry anthocyanins as novel antioxidants in human health and disease prevention. Molecular nutrition \& food research. 2007;51(6):675-83.

Zrenner E, Miliczek KD, Gabel VP, et al. The development of subretinal microphotodiodes for replacement of degenerated photoreceptors. Ophthalmic Res. 1997;29(5):269-80. 